\title{
Ni-Catalyzed Synthesis of Thiocarboxylic Acid Derivatives
}

\author{
John J. Monteith, Katerina Scotchburn, L. Reginald Mills and Sophie A. L. Rousseaux* \\ Davenport Research Laboratories, Department of Chemistry, University of Toronto \\ 80 St. George St., Toronto, ON, M5S 3H6
}

\section{Supporting Information 1: Experimental Data}

\section{Table of Contents}

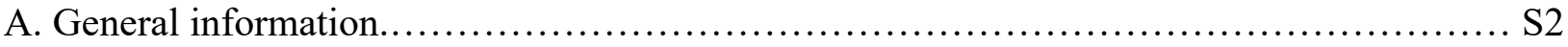

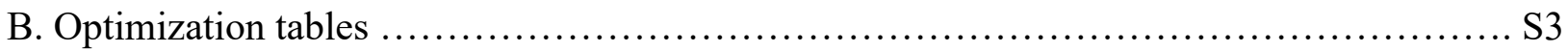

Table S1. Evaluation of ArZnCl equivalents, catalyst loadings, solvent ratios and

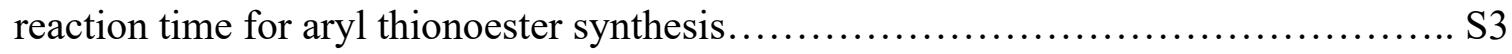

Table S2. Evaluation of Ni sources, ligands and solvents for aryl thionoester synthesis. S3 Table S3. Evaluation of Ni sources, ligands and reaction time for alkyl thionoester synthesis

Table S4. Evaluation of Ni sources, ligands and reaction time for alkyl thionoesters synthesis S4

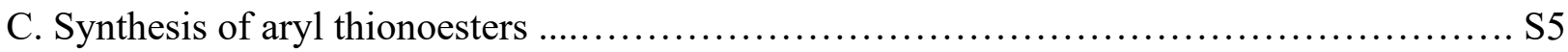

General procedure A: Preparation of aryl thionoesters from $O$-alkyl xanthates ......... S5

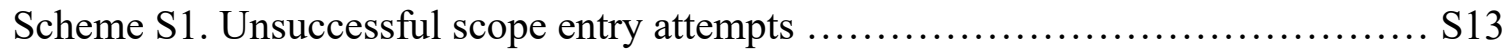

D. Synthesis of aryl thioamides ....................................................... S14

General procedure B: Preparation of aryl thioamides from thiocarbonyl imidazolides........

E. Synthesis of alkyl thionoesters and thioamides................................... S19

General procedure C: Synthesis of alkyl thionoesters and thioamides from thiocarbonyl

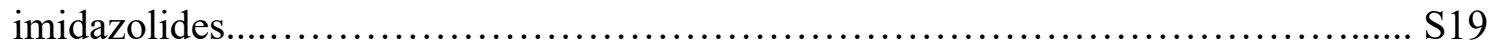

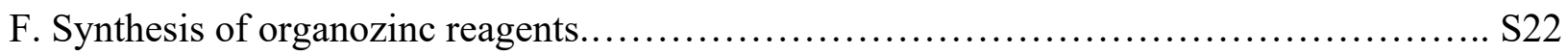

General procedure D: Preparation of organozinc reagents ..................... S22

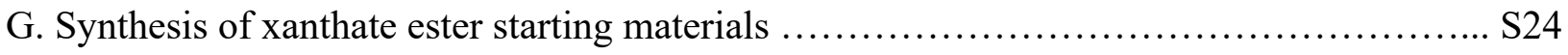

General procedure E: Preparation of $O$-alkyl xanthate starting materials.............S24

H. Synthesis of thiocarbonyl imidazolide starting materials......................... S30

General Procedure F: Synthesis of thiocarbonyl imidazolide starting materials......... S30

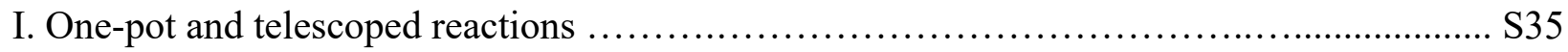

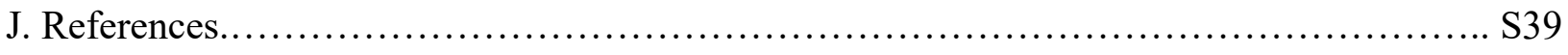




\section{A. General information}

Unless otherwise noted, all reactions were set up on the benchtop and run under an atmosphere of Ar or $\mathrm{N}_{2}$ using flame-dried glassware and anhydrous solvents. $\mathrm{CH}_{2} \mathrm{Cl}_{2}, \mathrm{Et}_{2} \mathrm{O}, \mathrm{MeCN} \mathrm{PhMe}$, and THF were purchased as HPLC-grade (inhibitor-free) from Caledon or Sigma-Aldrich, and were dried using a PureSolv MD 5 solvent purification system and used without further manipulation. 1,4-Dioxane was purchased as anhydrous from Sigma-Aldrich in a Sure/Seal bottle and was degassed by sparging with $\mathrm{N}_{2}$ for 15 min prior to use. $\mathrm{Ni}(\mathrm{acac})_{2} \bullet \mathrm{xH}_{2} \mathrm{O}$ was purchased from Sigma-Aldrich as $\mathrm{Ni}(\mathrm{acac})_{2}$ and was stored in a dessicator open to air, so was assumed to have a small amount of hydration and was denoted as " $\mathrm{Ni}(\text { acac })_{2} \bullet \mathrm{xH}_{2} \mathrm{O}$ ".

$\mathrm{Ni}(\mathrm{OAc})_{2} \bullet 4 \mathrm{H}_{2} \mathrm{O}$ was purchased from Sigma-Aldrich. Bathocuproine and Phenanthroline ligands were purchased from Oakwood Chemicals and terpyridine was purchased from Sigma-Aldrich. $\mathrm{Zn}(\mathrm{II})$ salts were purchased as anhydrous-grade from Sigma-Aldrich and were stored and weighed in a glovebox. All other commercial reagents were used as received. Compounds were purified by flash column chromatography using SiliCycle SilicaFlash P60 silica gel. The 8- and 16-mL threaded culture tubes used for reactions were purchased from Fisher (catalogue nos. 14957- 76A and 14-959-35A) and were sealed using size 19 rubber septa and electrical tape.

All products and starting materials were stored at $-20{ }^{\circ} \mathrm{C}$ under argon and away from light as a precaution. Arylzinc reagents were stored in culture tubes under argon at room temperature.

GC-MS data was obtained on a Shimadzu GCMS-QP2010 SE; yields represent peak areas calibrated against n-dodecane as an internal standard. ${ }^{1} \mathrm{H}$ and ${ }^{13} \mathrm{C}$ NMR spectra were recorded on Varian MercuryPlus $400 \mathrm{MHz}$, Agilent DD2 $500 \mathrm{MHz}$, or Bruker Advance III $400 \mathrm{MHz}$ spectrometers. The multiplicity denoted as "sxt" represents an apparent sextet. TLC samples were run on EMD Millipore TLC Silica gel 60 F254 plates and were visualized by UV or by staining with standard $\mathrm{KMnO} 4$, p-anisaldehyde, or vanillin stains. IR spectra were obtained on a Perkin-Elmer Spectrum 100 instrument equipped with a single-bounce diamond/ZnSe ATR accessory as solids or thin films. Melting points were obtained on a Fisher-Johns Melting Point Apparatus. High-resolution mass spectra (HRMS) were recorded on a JEOL AccuTOF JMST1000LV mass spectrometer equipped with a Direct Analysis in Real Time (DART) ion source. 


\section{B. Optimization}

Table S1. Evaluation of $\mathrm{ArZnCl}$ equivalents, catalyst loadings, solvent ratios and reaction time for aryl thionoester synthesis

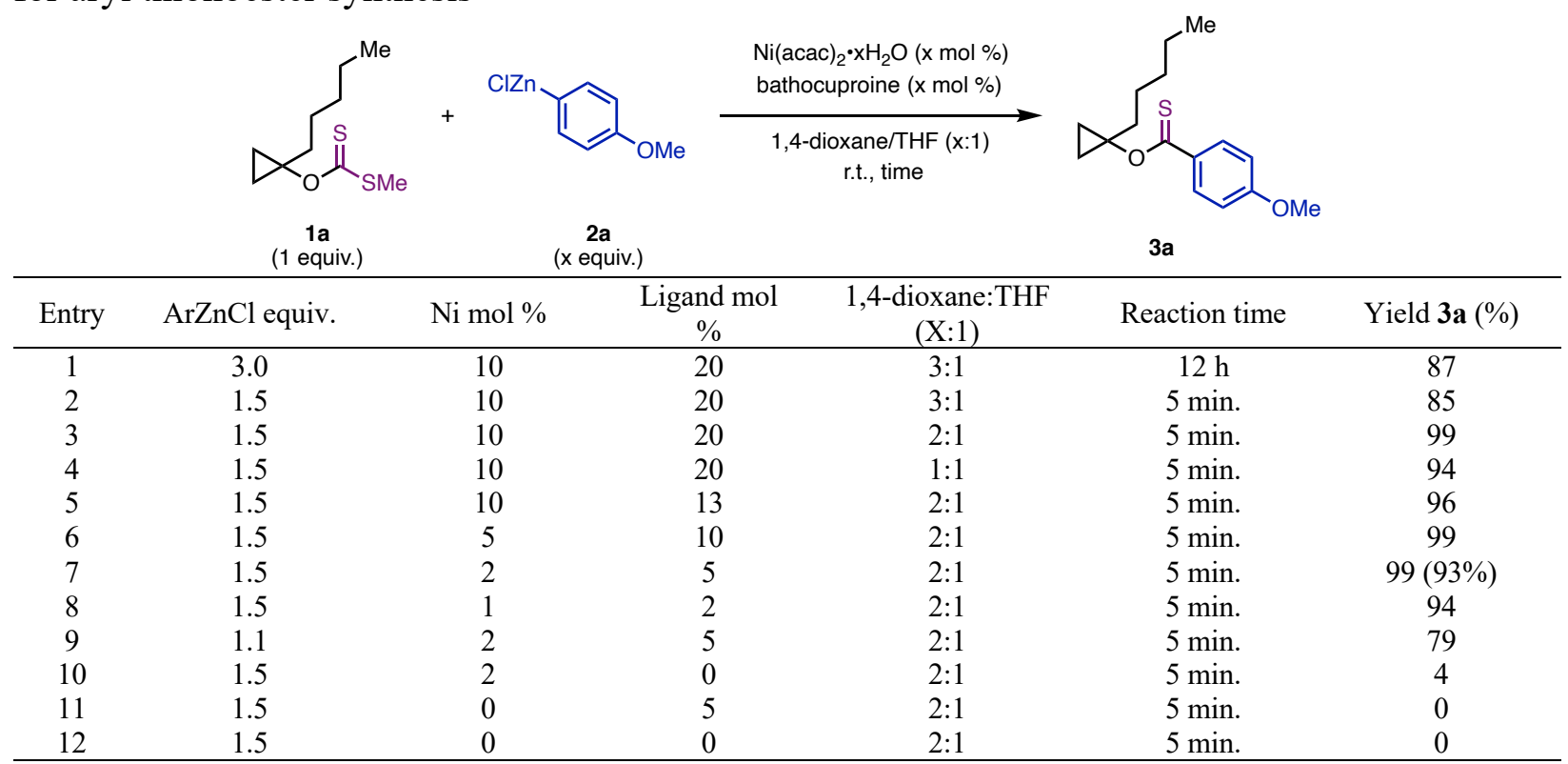

Yields determined by GC-MS using $n$-dodecane as internal standard. Isolated yields in brackets.

Table S2. Evaluation of Ni sources, ligands and solvents for aryl thionoester synthesis

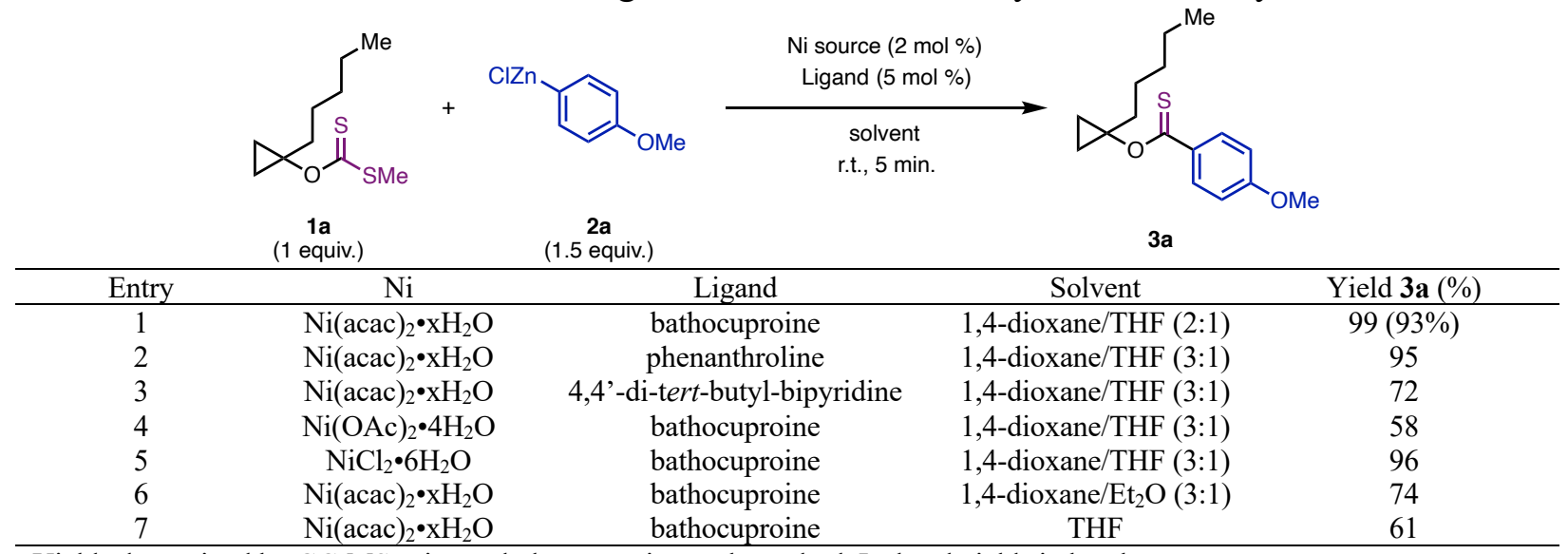

Yields determined by GC-MS using $n$-dodecane as internal standard. Isolated yields in brackets.

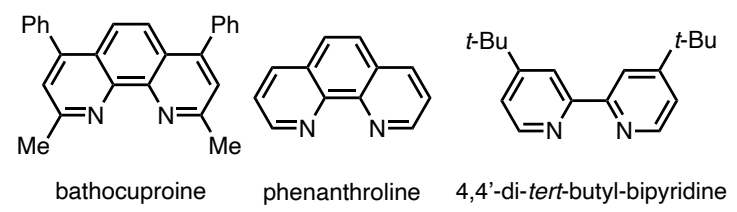


Table S3. Evaluation of Ni sources, ligands and reaction time for aryl thioamide synthesis

\begin{tabular}{|c|c|c|c|c|c|}
\hline & & (1 equiv.) & & & \\
\hline Entry & $\begin{array}{l}\mathrm{ArZnCl} \\
\text { equiv. }\end{array}$ & Ni source & Ligand & $\begin{array}{c}\text { Reaction } \\
\text { time }\end{array}$ & Yield 3a (\%) \\
\hline 1 & 1.5 & $\mathrm{Ni}(\mathrm{OAc})_{2} \bullet 4 \mathrm{H}_{2} \mathrm{O}(2 \mathrm{~mol} \%)$ & terpyridine $(5 \mathrm{~mol} \%)$ & $1 \mathrm{~h}$ & 0 \\
\hline 2 & 1.5 & $\mathrm{Ni}(\mathrm{OAc})_{2} \cdot 4 \mathrm{H}_{2} \mathrm{O}(2 \mathrm{~mol} \%)$ & bathocuprorine ( $5 \mathrm{~mol} \%$ ) & $1 \mathrm{~h}$ & 7 \\
\hline 3 & 1.5 & $\mathrm{Ni}(\mathrm{OAc})_{2} \cdot 4 \mathrm{H}_{2} \mathrm{O}(2 \mathrm{~mol} \%)$ & bpp $(5 \mathrm{~mol} \%)$ & $1 \mathrm{~h}$ & 0 \\
\hline 4 & 1.5 & $\mathrm{Ni}(\mathrm{OAc})_{2} \cdot 4 \mathrm{H}_{2} \mathrm{O}(2 \mathrm{~mol} \%)$ & 4,4'-di-tert-butyl-bipyridine ( $5 \mathrm{~mol} \%$ ) & $1 \mathrm{~h}$ & 9 \\
\hline 5 & 1.5 & $\mathrm{Ni}(\mathrm{OAc})_{2} \cdot 4 \mathrm{H}_{2} \mathrm{O}(2 \mathrm{~mol} \%)$ & phenanthroline $(5 \mathrm{~mol} \%)$ & $1 \mathrm{~h}$ & 44 \\
\hline 6 & 1.5 & $\mathrm{Ni}(\mathrm{OAc})_{2} \cdot 4 \mathrm{H}_{2} \mathrm{O}(2 \mathrm{~mol} \%)$ & pybox $(5 \mathrm{~mol} \%)$ & $1 \mathrm{~h}$ & 1 \\
\hline 7 & 3.0 & $\mathrm{Ni}(\mathrm{OAc})_{2} \cdot 4 \mathrm{H}_{2} \mathrm{O}(2 \mathrm{~mol} \%)$ & phenanthroline ( 5 mol \%) & $1 \mathrm{~h}$ & 49 \\
\hline 8 & 6.0 & $\mathrm{Ni}(\mathrm{OAc})_{2} \cdot 4 \mathrm{H}_{2} \mathrm{O}(2 \mathrm{~mol} \%)$ & phenanthroline $(5 \mathrm{~mol} \%)$ & $1 \mathrm{~h}$ & 70 \\
\hline 9 & 1.5 & $\mathrm{Ni}(\mathrm{OAc})_{2} \cdot 4 \mathrm{H}_{2} \mathrm{O}(2 \mathrm{~mol} \%)$ & 2,9-dimethylphenanthroline (5 mol \%) & $1 \mathrm{~h}$ & 15 \\
\hline 10 & 1.5 & $\mathrm{Ni}(\mathrm{OAc})_{2} \cdot 4 \mathrm{H}_{2} \mathrm{O}(2 \mathrm{~mol} \%)$ & 4,7-dimethylphenanthroline ( $5 \mathrm{~mol} \%$ ) & $1 \mathrm{~h}$ & 9 \\
\hline 11 & 1.5 & $\mathrm{Ni}(\mathrm{acac})_{2} \bullet \mathrm{xH}_{2} \mathrm{O}(2 \mathrm{~mol} \%)$ & phenanthroline $(5 \mathrm{~mol} \%)$ & $1 \mathrm{~h}$ & $91(89 \%)$ \\
\hline 12 & 1.5 & $\mathrm{NiCl}_{2} \cdot 6 \mathrm{H}_{2} \mathrm{O}(2 \mathrm{~mol} \%)$ & phenanthroline ( $5 \mathrm{~mol} \%)$ & $1 \mathrm{~h}$ & 79 \\
\hline 13 & 1.5 & $\mathrm{NiCl}_{2} \bullet$ glyme $(2 \mathrm{~mol} \%)$ & phenanthroline ( $5 \mathrm{~mol} \%)$ & $1 \mathrm{~h}$ & 79 \\
\hline 14 & 1.5 & $\mathrm{Ni}(\mathrm{acac})_{2} \bullet \mathrm{xH}_{2} \mathrm{O}(2 \mathrm{~mol} \%)$ & phenanthroline ( $5 \mathrm{~mol} \%)$ & $5 \mathrm{~min}$. & 73 \\
\hline 15 & 1.5 & $\mathrm{Ni}(\mathrm{acac})_{2} \cdot \mathrm{xH}_{2} \mathrm{O}(2 \mathrm{~mol} \%)$ & phenanthroline ( $5 \mathrm{~mol} \%)$ & $12 \mathrm{~h}$ & 90 \\
\hline 16 & 1.5 & $\mathrm{Ni}(\mathrm{acac})_{2} \cdot \mathrm{xH}_{2} \mathrm{O}(2 \mathrm{~mol} \%)$ & - & $1 \mathrm{~h}$ & 5 \\
\hline 17 & 1.5 & - & phenanthroline ( $5 \mathrm{~mol} \%)$ & $1 \mathrm{~h}$ & 0 \\
\hline 18 & 1.5 & - & - & $1 \mathrm{~h}$ & 0 \\
\hline
\end{tabular}

Yields determined by GC-MS using $n$-dodecane as internal standard. Isolated yields in brackets.

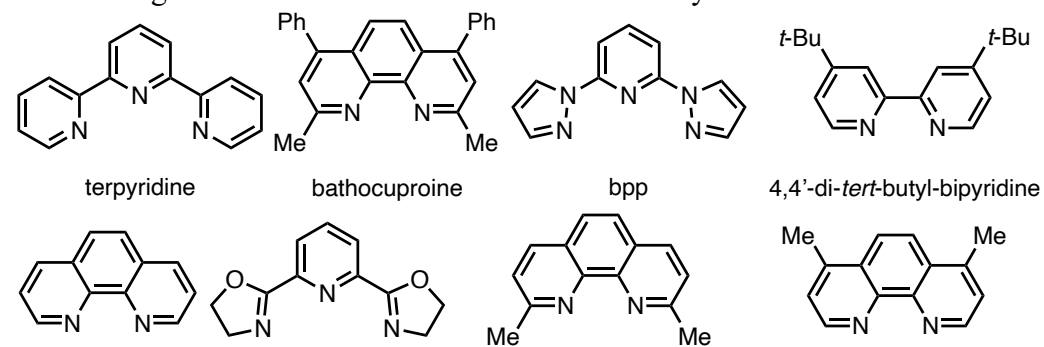

phenanthroline pybox 2,9-dimethylphenanthroline 4,7-dimethylphenanthroline

Table S4. Evaluation of Ni sources, ligands and reaction time for alkyl thionoester synthesis

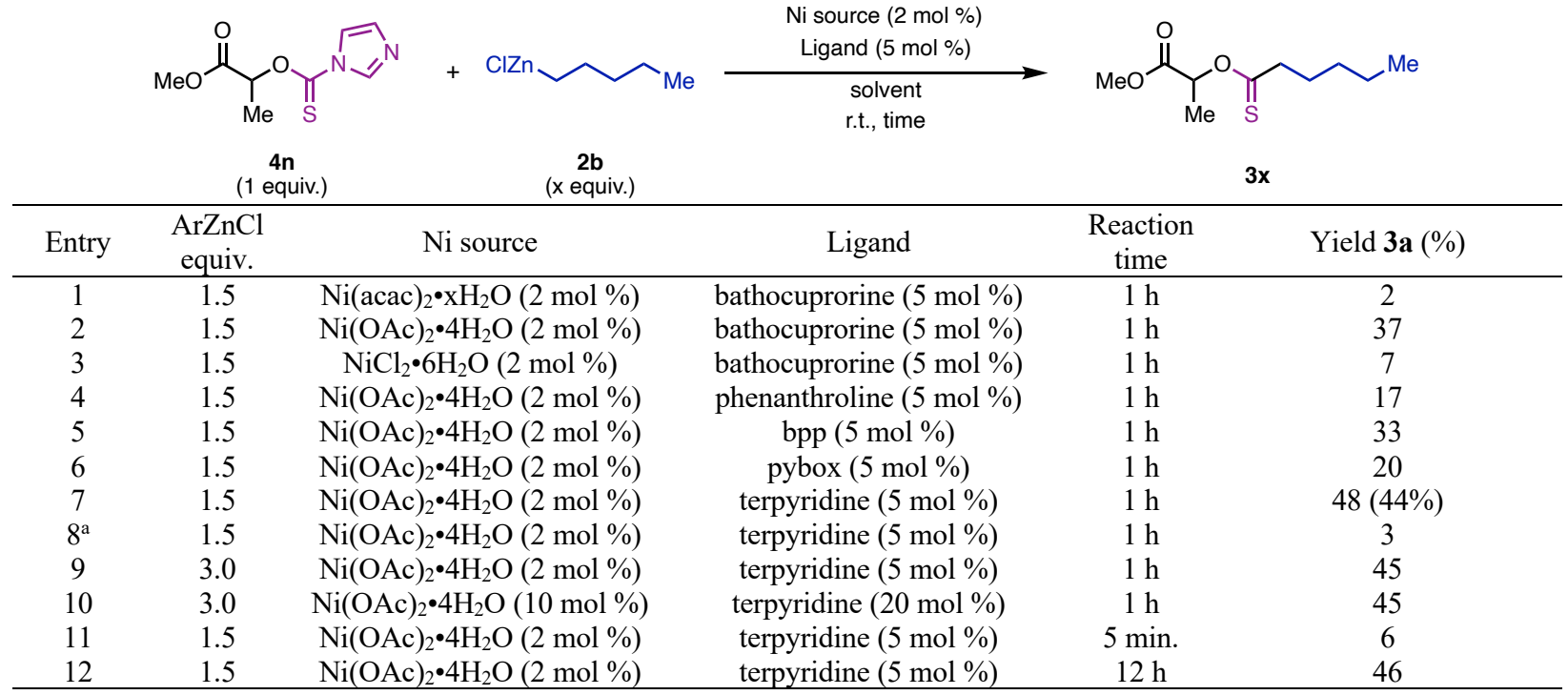

Yields determined by GC-MS using $n$-dodecane as internal standard. Isolated yields in brackets. ${ }^{a}$ using xanthate ester 10. 


\section{Synthesis of aryl thionoesters}

\section{General procedure A: Preparation of aryl thionoesters from $\boldsymbol{O}$-alkyl xanthates.}

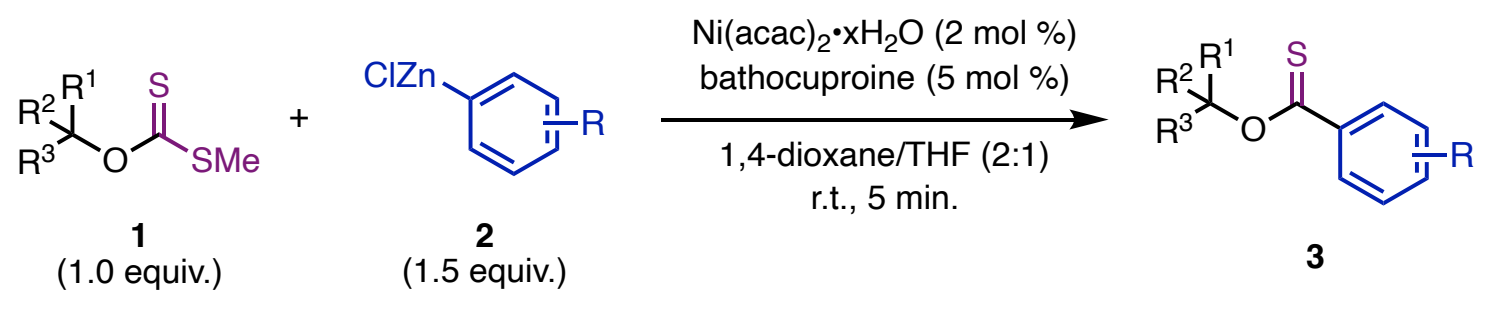

To a flame-dried 16-mL reaction tube with a stir bar was added xanthate substrate $1(0.20 \mathrm{mmol}$, 1.0 equiv), $\mathrm{Ni}(\mathrm{acac})_{2} \cdot \mathrm{xH}_{2} \mathrm{O}(1.0 \mathrm{mg}, 0.0040 \mathrm{mmol}, 2.0 \mathrm{~mol} \%)$, and bathocuproine (3.6 mg, $0.010 \mathrm{mmol}, 5 \mathrm{~mol} \%$ ). The flask was sealed with a septum, and evacuated and backfilled with $\mathrm{N}_{2}(\times 3)$. 1,4-Dioxane (anhydrous, degassed for 15 minutes by sparging with argon; an amount to reach a 2:1 1,4-dioxane/THF ratio, according to the volume of titrated arylzinc(II) chloride reagent required for $0.30 \mathrm{mmol}$ ) was added and the reaction mixture was stirred at r.t. for $10 \mathrm{~min}$. After $10 \mathrm{~min}$, while stirring, arylzinc solution $(0.30 \mathrm{mmol}$ of a solution in THF (typically 0.86 $\mathrm{mL}$ of a $0.35 \mathrm{M}$ solution), 3.0 equiv.) was added at once and the reaction was stirred at r.t. (23 $\left.{ }^{\circ} \mathrm{C}\right)$ for 5 minutes. The reaction was opened to air and quenched with sat. aq. $\mathrm{NH}_{4} \mathrm{Cl}(\sim 1.5 \mathrm{~mL})$. If analyzing by GC-MS, $n$-dodecane was added as an internal standard. EtOAc $(\sim 2 \mathrm{~mL})$ was added and the reaction mixture was mixed vigorously through pipetting. The organic layer was filtered through a Pasteur pipette loaded with $\mathrm{MgSO}_{4}$ and celite. An additional $2 \mathrm{~mL}$ of EtOAc was added to the remaining aqueous layer, vigorously mixed, and passed through the same $\mathrm{MgSO}_{4} /$ celite plug. (The sulfur-containing aqueous waste was treated with bleach and disposed of appropriately.) The combined organic phase was concentrated and the crude residue was purified by flash column chromatography to yield the desired thionoester product.<smiles>CCCCCC1(OC(=S)c2ccc(OC)cc2)CC1</smiles>

O-(1-pentylcyclopropyl) 4-methoxybenzothioate (3a). Prepared on 0.20-mmol scale following General Procedure A. The crude residue was purified by flash column chromatography (gradient of $0-30 \% \mathrm{PhMe} /$ hexanes) to yield the product as a dark-yellow oil (52 $\mathrm{mg}, 0.19 \mathrm{mmol}, 93 \%) .{ }^{1} \mathbf{H}$ NMR (500 MHz, CDCl $3,298 \mathrm{~K}): \delta_{\mathrm{H}} 8.16-8.09(\mathrm{~m}, 2 \mathrm{H}), 6.87-6.81(\mathrm{~m}, 2 \mathrm{H}), 3.85(\mathrm{~s}, 3 \mathrm{H}), 2.08-$ $2.01(\mathrm{~m}, 2 \mathrm{H}), 1.46(\mathrm{~m}, 2 \mathrm{H}), 1.33-1.25(\mathrm{~m}, 4 \mathrm{H}), 1.15-1.08(\mathrm{~m}, 2 \mathrm{H}), 0.85(\mathrm{~m}, 5 \mathrm{H}) \mathrm{ppm} .{ }^{13} \mathbf{C} \mathbf{~ N M R}$ $\left(126 \mathrm{MHz}, \mathrm{CDCl}_{3}, 298 \mathrm{~K}\right): \delta_{\mathrm{C}} 209.2,163.6,132.3,130.9,113.3,66.0,55.6,33.3,31.8,25.9$, 22.8, 14.2, $13.2 \mathrm{ppm}$. HRMS $\mathrm{m} / \mathrm{z}$ (DART): calculated for $\mathrm{C}_{16} \mathrm{H}_{23} \mathrm{O}_{2} \mathrm{~S}(\mathrm{M}+\mathrm{H}): 279.1413$; found: 279.1419; IR (neat): 3247, 3190, 3134, 3061, 2976, 2922, 2857, 2857, 1656, 1597, 1543, 1498, 1443, 1316, 1205, 1167, 1054, 1025, 738, 692, $628 \mathrm{~cm}^{-1} ; \mathbf{R}_{\mathbf{f}}(8: 2$ hexanes/PhMe; Vanillin): 0.70. 
Prepared on 1.0-mmol scale using General Procedure A with the following modifications: $\mathrm{Ni}(\mathrm{acac})_{2} \bullet \mathrm{xH}_{2} \mathrm{O}(5.1 \mathrm{mg}, 0.020 \mathrm{mmol}, 2.0 \mathrm{~mol} \%)$, bathocuproine (18 mg, $0.050 \mathrm{mmol}, 5.0 \mathrm{~mol}$ $\%$ ). The crude residue was purified by flash column chromatography (gradient of $0-30 \%$ $\mathrm{PhMe} / \mathrm{h}$ exanes to yield the product as a light-yellow oil (0.28 g, $1.0 \mathrm{mmol}, 99 \%)$.<smiles>COc1ccc(C(=S)OC2(P)CC2)cc1</smiles>

3b

$\boldsymbol{O}$-(1-phenylcyclopropyl) 4-methoxybenzothioate (3b). Prepared on 0.20-mmol scale following General Procedure A. The crude residue was purified by flash column chromatography (gradient of $0-30 \% \mathrm{PhMe} /$ hexanes) to yield the product as a yellow oil (51 mg, $0.18 \mathrm{mmol}, 90 \%)$.

${ }^{1} \mathbf{H}$ NMR $\left(500 \mathrm{MHz}, \mathrm{CDCl}_{3}, 298 \mathrm{~K}\right): \delta_{\mathrm{H}} 8.26-8.18(\mathrm{~m}, 2 \mathrm{H}), 7.32-7.27(\mathrm{~m}, 2 \mathrm{H}), 7.26-7.23(\mathrm{~m}$, 2H), 7.22-7.16 (m, 1H), 6.92-6.84 (m, 2H), $3.87(\mathrm{~s}, 3 \mathrm{H}), 1.61-1.57(\mathrm{~m}, 2 \mathrm{H}), 1.50-1.43(\mathrm{~m}, 2 \mathrm{H})$ ppm. ${ }^{13} \mathbf{C}$ NMR $\left(126 \mathrm{MHz}, \mathrm{CDCl}_{3}, 298 \mathrm{~K}\right): \delta_{\mathrm{C}} 208.6,163.8,140.0,131.9,131.1,128.4,126.8$, 125.2, 113.4, 65.3, 55.7, 17.4 ppm. HRMS $m / z$ (DART): calculated for $\mathrm{C}_{17} \mathrm{H}_{17} \mathrm{O}_{2} \mathrm{~S}(\mathrm{M}+\mathrm{H})$ : 285.0944; found: 285.0953; IR (neat): 3085, 3011, 2964, 2928, 2835, 1598, 1501, 1450, 1421, $1325,1280,1253,1183,1169,1113,1020,839,686,695 \mathrm{~cm}^{-1} ; \mathbf{R}_{\mathbf{f}}(9: 1$ hexanes/PhMe;

Vanillin): 0.20 .<smiles>COc1ccc(C(=S)OC(C)Cc2ccccc2)cc1</smiles>

3c

$O$-(1-phenylpropan-2-yl) 4-methoxybenzothioate (3c). Prepared on 0.20-mmol scale following General Procedure A. The crude residue was purified by flash column chromatography (gradient of $0-60 \% \mathrm{PhMe} /$ hexanes) to yield the product as a yellow oil (56 mg, $0.19 \mathrm{mmol}, 97 \%)$.

${ }^{1} \mathbf{H}$ NMR $\left(500 \mathrm{MHz}, \mathrm{CDCl}_{3}, 298 \mathrm{~K}\right): \delta_{\mathrm{H}} 8.17-8.12(\mathrm{~m}, 2 \mathrm{H}), 7.29(\mathrm{~m}, 4 \mathrm{H}), 7.25-7.21(\mathrm{~m}, 1 \mathrm{H})$, 6.89-6.83 (m, 2H), 6.04-5.98 (m, 1H), $3.86(\mathrm{~s}, 3 \mathrm{H}), 3.25(\mathrm{dd}, J=13.7,5.8 \mathrm{~Hz}, 1 \mathrm{H}), 2.97(\mathrm{dd}, J$ $=13.7,7.0 \mathrm{~Hz}, 1 \mathrm{H}), 1.41(\mathrm{~d}, J=6.3 \mathrm{~Hz}, 3 \mathrm{H}) \mathrm{ppm} .{ }^{13} \mathbf{C} \mathbf{N M R}\left(126 \mathrm{MHz}, \mathrm{CDCl}_{3}, 298 \mathrm{~K}\right): \delta_{\mathrm{C}}$ 209.8, 163.7, 137.4, 132.2, 131.2, 129.7, 128.6, 126.7, 113.3, 78.8, 55.7, 41.8, 18.8 ppm. HRMS $\mathrm{m} / z$ (DART): calculated for $\mathrm{C}_{17} \mathrm{H}_{19} \mathrm{O}_{2} \mathrm{~S}(\mathrm{M}+\mathrm{H}): 287.1100$; found: 287.1106 ; IR (neat): 3067 , 3006, 2984, 2929, 2834, 1593, 1498, 1450, 1421, 1323, 1223, 1207, 1168, 1113, 1057, 1018, 833, 699, $639 \mathrm{~cm}^{-1} ; \mathbf{R}_{\mathbf{f}}(5: 5$ hexanes/PhMe; Vanillin): 0.61.<smiles>COc1ccc(C(=S)OC2CCCCC2)cc1</smiles>

3d

$\boldsymbol{O}$-cyclohexyl 4-methoxybenzothioate (3d). Prepared on 0.20-mmol scale following General Procedure A. The crude residue was purified by flash column chromatography (gradient of 0 $30 \% \mathrm{PhMe} /$ hexanes) to yield the product as a yellow oil (46 mg, $0.18 \mathrm{mmol}, 91 \%) .{ }^{1} \mathbf{H}$ NMR 
$\left(500 \mathrm{MHz}, \mathrm{CDCl}_{3}, 298 \mathrm{~K}\right): \delta_{\mathrm{H}} 8.22-8.13(\mathrm{~m}, 2 \mathrm{H}), 6.94-6.83(\mathrm{~m}, 2 \mathrm{H}), 5.68(\mathrm{tt}, J=8.4,3.8 \mathrm{~Hz}$, $1 \mathrm{H}), 3.86(\mathrm{~s}, 3 \mathrm{H}), 2.06-2.00(\mathrm{~m}, 2 \mathrm{H}), 1.84-1.77(\mathrm{~m}, 2 \mathrm{H}), 1.74-1.69(\mathrm{~m}, 2 \mathrm{H}), 1.68-1.57(\mathrm{~m}, 1 \mathrm{H})$, $1.50-1.36(\mathrm{~m}, 3 \mathrm{H}) \mathrm{ppm} .{ }^{13} \mathbf{C} \mathbf{N M R}\left(126 \mathrm{MHz}, \mathrm{CDCl}_{3}, 298 \mathrm{~K}\right): \delta_{\mathrm{C}} 209.8,163.6,132.4,131.2$, 113.3, 79.8, 55.6, 31.0, 25.6, $23.8 \mathrm{ppm}$. HRMS $m / z$ (DART): calculated for $\mathrm{C}_{14} \mathrm{H}_{19} \mathrm{O}_{2} \mathrm{~S}(\mathrm{M}+\mathrm{H})$ : 251.1100; found: 251.1102; IR (neat): 3007, 2931, 2856, 1598, 1574, 1505, 1450, 1420, 1319, 1248, 1217, 1165, 1110, 1022, $634 \mathrm{~cm}^{-1} ; \mathbf{R}_{\mathbf{f}}(5: 5$ hexanes/PhMe; Vanillin): 0.71.<smiles>COc1ccc(C(=S)OC(C)CC(=O)Nc2ccccc2)cc1</smiles>

$3 e$

O-(4-oxo-4-(phenylamino)butan-2-yl) 4-methoxybenzothioate (3e). Prepared on 0.20-mmol scale following General Procedure A with the following modifications: 3.0 equivalents $(0.6$ mmol) of $\mathrm{ArZnCl}$ was used instead of 1.5 equivalents. The crude residue was purified by flash column chromatography (gradient of $0-20 \%$ EtOAc/hexanes) to yield the product as a yellow solid (38 mg, $0.11 \mathrm{mmol}, 57 \%) .{ }^{1} \mathbf{H}$ NMR (500 MHz, $\left.\mathrm{CDCl}_{3}, 298 \mathrm{~K}\right): \delta_{\mathrm{H}} 8.20-8.14(\mathrm{~m}, 2 \mathrm{H})$, 7.61 (s (br), 1H), 7.51-7.44 (m, 2H), 7.33-7.28 (m, 2H), 7.13-7.08 (m, 1H), 6.89-6.81 (m, 2H), 6.15-6.09 (m, 1H), $3.86(\mathrm{~s}, 3 \mathrm{H}), 3.04$ (dd, $J=14.3,5.3 \mathrm{~Hz}, 1 \mathrm{H}), 2.76$ (dd, $J=14.3,6.6 \mathrm{~Hz}, 1 \mathrm{H})$, $1.62(\mathrm{~d}, J=6.3 \mathrm{~Hz}, 3 \mathrm{H}) \mathrm{ppm} .{ }^{13} \mathbf{C} \mathbf{N M R}\left(126 \mathrm{MHz}, \mathrm{CDCl}_{3}, 298 \mathrm{~K}\right): \delta_{\mathrm{C}} 209.5,167.5,163.9$, 137.7, 131.5, 131.2, 129.0, 124.4, 119.9, 113.4, 75.3, 55.6, 43.9, $19.5 \mathrm{ppm}$. HRMS m/z (DART): calculated for $\mathrm{C}_{18} \mathrm{H}_{20} \mathrm{NO}_{3} \mathrm{~S}(\mathrm{M}+\mathrm{H}): 330.1158$; found: 330.1156; IR (neat): 3270, 3014, 2982, 2944, 2839, 1729, 1650, 1598, 1533, 1497, 1444, 1419, 1377, 1317, 1276, 1169, 1215, 1169, 1111, 1063, 1014, 842, 722, 688, $594 \mathrm{~cm}^{-1} ; \mathbf{R}_{\mathbf{f}}$ (95:5 hexanes/EtOAc; Vanillin): 0.31.<smiles>COc1ccc(C(=S)OCCCc2ccccc2)cc1</smiles>

3f

$\boldsymbol{O}$-(3-phenylpropyl) 4-methoxybenzothioate (3f). Prepared on 0.20 -mmol scale following General Procedure A. The crude residue was purified by flash column chromatography (gradient of $0-10 \% \mathrm{PhMe} /$ hexanes) to yield the product as a yellow oil $(49 \mathrm{mg}, 0.17 \mathrm{mmol}, 83 \%) .{ }^{1} \mathbf{H}$ NMR (500 MHz, $\left.\mathrm{CDCl}_{3}, 298 \mathrm{~K}\right): \delta_{\mathrm{H}} 8.21-8.13(\mathrm{~m}, 2 \mathrm{H}), 7.34-7.29(\mathrm{~m}, 2 \mathrm{H}), 7.24-7.17(\mathrm{~m}, 3 \mathrm{H})$, 6.91-6.83 (m, 2H), $4.68(\mathrm{t}, J=6.4 \mathrm{~Hz}, 2 \mathrm{H}), 3.87(\mathrm{~s}, 3 \mathrm{H}), 2.86-2.83(\mathrm{~m}, 2 \mathrm{H}), 2.29-2.20(\mathrm{~m}, 2 \mathrm{H})$ ppm. ${ }^{13} \mathbf{C}$ NMR $\left(126 \mathrm{MHz}, \mathrm{CDCl}_{3}, 298 \mathrm{~K}\right): \delta_{\mathrm{C}} 210.6,164.1,141.3,131.7,131.1,128.7,128.6$, 126.2 , 113.4, 71.7, 55.6, 32.6, $30.2 \mathrm{ppm}$. HRMS $\mathrm{m} / \mathrm{z}$ (DART): calculated for $\mathrm{C}_{17} \mathrm{H}_{19} \mathrm{O}_{2} \mathrm{~S}(\mathrm{M}+\mathrm{H})$ : 287.1100; found: 287.1104; IR (neat): 3067, 3028, 2933, 2842, 1598, 1503, 1455, 1325, 1275, 1251, 1218, 1165, 1111, 1027, 837, 822, 698, 634, $571 \mathrm{~cm}^{-1} ; \mathbf{R}_{\mathbf{f}}(95: 5$ hexanes/PhMe; Vanillin): 0.88 . 


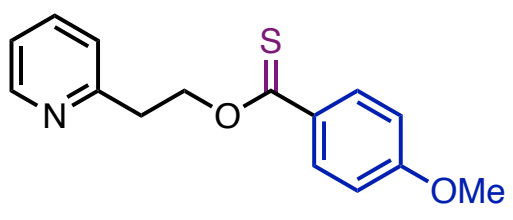

$3 g$

$\boldsymbol{O}$-(2-(pyridin-2-yl)ethyl) 4-methoxybenzothioate (3g). Prepared on 0.20-mmol scale following General Procedure A. The crude residue was purified by flash column chromatography (gradient of $0-30 \% \mathrm{PhMe} /$ hexanes $)$ to yield the product as a yellow oil $(33 \mathrm{mg}, 0.12 \mathrm{mmol}, 60 \%) .{ }^{1} \mathbf{H}$ NMR $\left(500 \mathrm{MHz}, \mathrm{CDCl}_{3}, 298 \mathrm{~K}\right): \delta_{\mathrm{H}} 8.49(\mathrm{~d}, J=4.9 \mathrm{~Hz}, 1 \mathrm{H}), 8.14-8.07(\mathrm{~m}, 2 \mathrm{H}), 7.65(\mathrm{td}, J=$ 7.7, $1.8 \mathrm{~Hz}, 1 \mathrm{H}), 7.28(\mathrm{~d}, J=7.8 \mathrm{~Hz}, 1 \mathrm{H}), 7.19(\mathrm{dd}, J=7.4,5.1 \mathrm{~Hz}, 1 \mathrm{H}), 6.87-6.80(\mathrm{~m}, 2 \mathrm{H})$, $5.05(\mathrm{t}, J=6.7 \mathrm{~Hz}, 2 \mathrm{H}), 3.85(\mathrm{~s}, 3 \mathrm{H}), 3.40(\mathrm{t}, J=6.6 \mathrm{~Hz}, 2 \mathrm{H}) .{ }^{13} \mathbf{C}$ NMR $\left(101 \mathrm{MHz}, \mathrm{CDCl}_{3}, 298\right.$ $\mathrm{K}): \delta_{\mathrm{C}} 210.4,163.8,158.2,149.7,136.7,131.7,131.1,123.6,121.9,113.4,71.2,55.6,37.2 \mathrm{ppm}$. HRMS $m / z$ (DART): calculated for $\mathrm{C}_{15} \mathrm{H}_{16} \mathrm{NO}_{2} \mathrm{~S}(\mathrm{M}+\mathrm{H}): 274.0896$; found: 274.0893; IR (neat): 3088, 3003, 2925, 2837, 1595, 1506, 1437, 1324, 1294, 1223, 1168, 1115 ,1049, 830, 694, 585 $\mathrm{cm}^{-1} ; \mathbf{R}_{\mathbf{f}}(8: 2$ hexanes/EtOAc; Vanillin): 0.23.<smiles>C=CCCOC(=S)c1ccc(OC)cc1</smiles>

$3 \mathrm{~h}$

$\boldsymbol{O}$-(but-3-en-1-yl) 4-methoxybenzothioate (3h). Prepared on 0.20-mmol scale following General Procedure A. The crude residue was purified by flash column chromatography (gradient of $0-20 \% \mathrm{PhMe} /$ hexanes) to yield the product as a yellow oil (42 $\mathrm{mg}, 0.19 \mathrm{mmol}, 93 \%) .{ }^{1} \mathbf{H}$ NMR $\left(500 \mathrm{MHz}, \mathrm{CDCl}_{3}, 298 \mathrm{~K}\right): \delta_{\mathrm{H}} 8.21-8.14(\mathrm{~m}, 2 \mathrm{H}), 6.90-6.83(\mathrm{~m}, 2 \mathrm{H}), 5.92(\mathrm{ddt}, J=17.0$, $10.2,6.7 \mathrm{~Hz}, 1 \mathrm{H}), 5.21(\mathrm{ddt}, J=17.1,3.0,1.6 \mathrm{~Hz}, 1 \mathrm{H}), 5.14(\mathrm{ddt}, J=10.3,3.0,1.4 \mathrm{~Hz}, 1 \mathrm{H}), 4.70$ $(\mathrm{t}, J=6.6 \mathrm{~Hz}, 2 \mathrm{H}), 3.86(\mathrm{~s}, 3 \mathrm{H}), 2.66(\mathrm{dtdd} J=6.7,6.6,1.6,1.4 \mathrm{~Hz}, 2 \mathrm{H}) \mathrm{ppm} .{ }^{13} \mathbf{C}$ NMR $(126$ $\left.\mathrm{MHz}, \mathrm{CDCl}_{3}, 298 \mathrm{~K}\right): \delta_{\mathrm{C}} 210.6,163.8,134.2,131.7,131.1,117.6,113.4,71.2,55.6,32.9 \mathrm{ppm}$. HRMS $m / z$ (DART): calculated for $\mathrm{C}_{12} \mathrm{H}_{15} \mathrm{O}_{2} \mathrm{~S}(\mathrm{M}+\mathrm{H})$ : 223.0787; found: 223.0790; IR (neat): 3081, 3011, 2944, 2838, 1641, 1598, 1574, 1505, 1421, 1323, 1278, 1252, 1216, 1166, 1111, 1055, 1027, 918, 837, 633, $575 \mathrm{~cm}^{-1} ; \mathbf{R}_{\mathbf{f}}(8: 2$ hexanes/PhMe; Vanillin): 0.54.<smiles>C#CCCOC(=S)c1ccc(OC)cc1</smiles>

$3 \mathbf{i}$

O-(but-3-yn-1-yl) 4-methoxybenzothioate (3i). Prepared on 0.20-mmol scale following General Procedure A. The crude residue was purified by flash column chromatography (gradient of 0 $30 \% \mathrm{PhMe} /$ hexanes) to yield the product as a yellow oil (31 $\mathrm{mg}, 0.14 \mathrm{mmol}, 71 \%) .{ }^{1} \mathbf{H} \mathbf{~ N M R}$ $\left(500 \mathrm{MHz}, \mathrm{CDCl}_{3}, 298 \mathrm{~K}\right): \delta_{\mathrm{H}} 8.24-8.17(\mathrm{~m}, 2 \mathrm{H}), 6.91-6.84(\mathrm{~m}, 2 \mathrm{H}), 4.76(\mathrm{t}, J=6.7 \mathrm{~Hz}, 2 \mathrm{H})$, $3.87(\mathrm{~s}, 3 \mathrm{H}), 2.80(\mathrm{td}, J=6.7,2.7 \mathrm{~Hz}, 2 \mathrm{H}), 2.05$ (t, $J=2.7 \mathrm{~Hz}, 1 \mathrm{H}) \mathrm{ppm} .{ }^{13} \mathbf{C}$ NMR $(126 \mathrm{MHz}$, $\left.\mathrm{CDCl}_{3}, 298 \mathrm{~K}\right): \delta_{\mathrm{C}} 210.2,163.9,131.5,131.3,113.5,80.2,70.3,69.4,55.7,18.9$ ppm. HRMS $m / z$ (DART): calculated for $\mathrm{C}_{12} \mathrm{H}_{13} \mathrm{O}_{2} \mathrm{~S}(\mathrm{M}+\mathrm{H}): 221.0631$; found: 221.0639; IR (neat): 3290, 
$3261,2972,2836,1597,1571,1504,1461,1435,1368,1325,1582,1252,1218,1161,1112$, 1055, 1018, 830, 680, $584 \mathrm{~cm}^{-1} ; \mathbf{R}_{\mathbf{f}}$ (9:1 hexanes/PhMe; Vanillin): 0.40 .<smiles>[M]C(OC(=S)c1ccc(OC)cc1)c1ccc(Br)cc1</smiles>

3j

$O$-(1-(4-bromophenyl)ethyl) 4-methoxybenzothioate (3j). Prepared on 0.20-mmol scale following General Procedure A. The crude residue was purified by flash column chromatography (gradient of $0-5 \% \mathrm{PhMe} /$ hexanes) to yield the product as a yellow oil (53 $\mathrm{mg}, 0.16 \mathrm{mmol}, 78 \%$ ). ${ }^{1} \mathbf{H}$ NMR $\left(500 \mathrm{MHz}, \mathrm{CDCl}_{3}, 298 \mathrm{~K}\right): \delta_{\mathrm{H}} 8.25-8.15(\mathrm{~m}, 2 \mathrm{H}), 7.52-7.46(\mathrm{~m}, 2 \mathrm{H}), 7.34-7.26(\mathrm{~m}$, $2 \mathrm{H}), 6.92-6.83(\mathrm{~m}, 2 \mathrm{H}), 6.70(\mathrm{q}, J=6.6 \mathrm{~Hz}, 1 \mathrm{H}), 3.87(\mathrm{~s}, 3 \mathrm{H}), 1.73(\mathrm{~d}, J=6.6 \mathrm{~Hz}, 3 \mathrm{H}) \mathrm{ppm} .{ }^{13} \mathbf{C}$ NMR (126 MHz, $\left.\mathrm{CDCl}_{3}, 298 \mathrm{~K}\right): \delta_{\mathrm{C}} 209.0,163.9,140.5,131.9,131.8,131.3,128.2,121.9$, 113.5, 78.5, 55.7, 22.0 ppm. HRMS $m / z$ (DART): calculated for $\mathrm{C}_{16} \mathrm{H}_{16} \mathrm{BrO}_{2} \mathrm{~S}(\mathrm{M}+\mathrm{H})$ : 351.0049; found: 351.0051; IR (neat): 3359, 3197, 2965, 2918, 2850, 1661, 1632, 1599, 1506, $1462,1325,1256,1216,1167,1026,1010,837,822,704,635,565 \mathrm{~cm}^{-1} ; \mathbf{R}_{\mathbf{f}} 95: 5$ hexanes/PhMe; Vanillin): 0.25 .<smiles>[M]C(OC(=S)c1ccc(OC)cc1)c1ccccc1</smiles>

3k

$\boldsymbol{O}$-(1-phenylethyl) 4-methoxybenzothioate (3k). Prepared on 0.20-mmol scale following General Procedure A. The crude residue was purified by flash column chromatography (gradient of $0-30 \% \mathrm{PhMe} / \mathrm{hexanes}$ ) to yield the product as a yellow oil (44 $\mathrm{mg}, 0.16 \mathrm{mmol}, 81 \%) .{ }^{1} \mathbf{H}$ NMR (400 MHz, $\left.\mathrm{CDCl}_{3}, 298 \mathrm{~K}\right): \delta_{\mathrm{H}} 8.26-8.17(\mathrm{~m}, 2 \mathrm{H}), 7.47-7.42(\mathrm{~m}, 2 \mathrm{H}), 7.39-7.34$ (m, 2H), 7.33-7.28 (m, 1H), 6.91-6.83 (m, 2H), $6.77(\mathrm{q}, J=6.5 \mathrm{~Hz}, 1 \mathrm{H}), 3.86(\mathrm{~s}, 3 \mathrm{H}), 1.76(\mathrm{~d}, J=6.5 \mathrm{~Hz}$, 3H) ppm. ${ }^{13} \mathrm{C}$ NMR $\left(100 \mathrm{MHz}, \mathrm{CDCl}_{3}, 298 \mathrm{~K}\right): \delta_{\mathrm{C}} 209.3,163.8,141.5,132.1,131.3,128.7$, 128.1, 126.5, 113.4, 79.3, 55.7, $22.0 \mathrm{ppm}$. HRMS $m / z$ (DART): calculated for $\mathrm{C}_{16} \mathrm{H}_{17} \mathrm{O}_{2} \mathrm{~S}$ (M+H): 273.0944; found: 273.0946; IR (neat): 3067, 3035, 2983, 2929, 2838, 1598, 1574, 1504, 1451, 1420, 1323, 1253, 1214, 1165, 1111, 1058, 1024, 837, $696 \mathrm{~cm}^{-1} ; \mathbf{R}_{\mathbf{f}} 7: 3$ hexanes/PhMe; Vanillin): 0.27 .<smiles>COc1ccc(C(=S)OC(Cl)c2ccccc2)cc1</smiles>

31

$O$-(cyclohexyl(phenyl)methyl) 4-methoxybenzothioate (3I). Prepared on 0.20-mmol scale following General Procedure A. The crude residue was purified by flash column chromatography (gradient of $0-5 \% \mathrm{PhMe} /$ hexanes) to yield the product as a yellow oil (40 $\mathrm{mg}, 0.12 \mathrm{mmol}, 58 \%$ ). ${ }^{1} \mathbf{H}$ NMR $\left(500 \mathrm{MHz}, \mathrm{CDCl}_{3}, 298 \mathrm{~K}\right): \delta_{\mathrm{H}} 8.28-8.18(\mathrm{~m}, 2 \mathrm{H}), 7.36-7.30(\mathrm{~m}, 4 \mathrm{H}), 7.26-7.24(\mathrm{~m}$, $1 \mathrm{H}), 6.93-6.85$ (m, 2H), 6.43 (d, $J=7.4 \mathrm{~Hz}, 1 \mathrm{H}), 3.86$ (s, 3H), 2.06 (tdt, $J=10.9,6.8,3.3 \mathrm{~Hz}$, 
1H), 1.98-1.87 (m, 1H), 1.81-1.68 (m, 2H), 1.68-1.62 (m, 1H), 1.56-1.51 (m, 1H), 1.27-1.04 (m, 6H) ppm. ${ }^{13} \mathbf{C}$ NMR $\left(126 \mathrm{MHz}, \mathrm{CDCl}_{3}, 298 \mathrm{~K}\right): \delta_{\mathrm{C}} 209.5,163.7,139.1,132.1,131.2,128.3$, 127.8, 127.6, 113.4, 87.2, 55.7, 43.7, 29.3, 29.1, 26.5, 26.1, $26.1 \mathrm{ppm}$. HRMS $m / z$ (DART): calculated for $\mathrm{C}_{21} \mathrm{H}_{25} \mathrm{O}_{2} \mathrm{~S}(\mathrm{M}+\mathrm{H})$ : 341.1570; found: 341.1569; IR (neat): 3021, 2927, 2851, $1715,1596,1504,1447,1327,1272,1253,1215,1166,1043,1019,834,758,698,565 \mathrm{~cm}^{-1} ; \mathbf{R}_{\mathbf{f}}$ 95:5 hexanes/PhMe; Vanillin): 0.48.<smiles>COc1ccc(C(=S)OCc2ccccc2)cc1</smiles>

$3 m$

$\boldsymbol{O}$-benzyl 4-methoxybenzothioate (3m). Prepared on 0.20-mmol scale following General Procedure A. The crude residue was purified by flash column chromatography (gradient of 0 $40 \% \mathrm{PhMe} /$ hexanes) to yield the product as a yellow solid (45 $\mathrm{mg}, 0.17 \mathrm{mmol}, 87 \%) .{ }^{1} \mathbf{H}$ NMR (500 MHz, $\left.\mathrm{CDCl}_{3}, 298 \mathrm{~K}\right): \delta_{\mathrm{H}} 8.25-8.19(\mathrm{~m}, 2 \mathrm{H}), 7.51-7.46(\mathrm{~m}, 2 \mathrm{H}), 7.46-7.33(\mathrm{~m}, 3 \mathrm{H}), 6.90$ $6.83(\mathrm{~m}, 2 \mathrm{H}), 5.70(\mathrm{~s}, 2 \mathrm{H}), 3.86(\mathrm{~s}, 3 \mathrm{H}) \mathrm{ppm} .{ }^{13} \mathbf{C}$ NMR $\left(126 \mathrm{MHz}, \mathrm{CDCl}_{3}, 298 \mathrm{~K}\right): \delta_{\mathrm{C}} 210.2$, $163.9,135.7,131.6,131.3,128.8,128.5,128.5,113.4,73.9,55.7 \mathrm{ppm}$. HRMS $m / z$ (DART): calculated for $\mathrm{C}_{15} \mathrm{H}_{15} \mathrm{O}_{2} \mathrm{~S}(\mathrm{M}+\mathrm{H}): 259.0787$; found: 259.0795; IR (neat): 3023, 2944, 2935, 2936, 1729, 1607, 1509 1456, 1244, 1169, 1029, 819, $786 \mathrm{~cm}^{-1} ; \mathbf{R}_{\mathbf{f}}$ 7:3 hexanes/PhMe; Vanillin): 0.59 .<smiles>COc1ccc(C(=S)OCc2ccc(Cl)cc2)cc1</smiles>

$3 n$

$\boldsymbol{O}$-(4-chlorobenzyl) 4-methoxybenzothioate (3n). Prepared on 0.20-mmol scale following General Procedure A. The crude residue was purified by flash column chromatography (gradient of $0-20 \% \mathrm{PhMe} / \mathrm{hexanes}$ ) to yield the product as a yellow oil (45 $\mathrm{mg}, 0.15 \mathrm{mmol}, 77 \%) .{ }^{1} \mathbf{H}$ NMR (500 MHz, $\left.\mathrm{CDCl}_{3}, 298 \mathrm{~K}\right): \delta_{\mathrm{H}} 8.21-8.18(\mathrm{~m}, 2 \mathrm{H}), 7.42-7.37$ (m, 4H), 6.88-6.85 (m, 2H), 5.66 (s, 2H), 3.86 (s, 3H) ppm. ${ }^{13} \mathbf{C}$ NMR (126 MHz, CDCl $\left.3,298 \mathrm{~K}\right): \delta_{\mathrm{C}} 210.0,164.0,134.5$, 134.2, 131.5, 131.3, 129.8, 129.0, 113.5, 72.9, $55.7 \mathrm{ppm}$. HRMS $\mathrm{m} / \mathrm{z}$ (DART): calculated for $\mathrm{C}_{15} \mathrm{H}_{14} \mathrm{ClO}_{2} \mathrm{~S}(\mathrm{M}+\mathrm{H})$ : 293.0398; found: 293.0393; IR (neat): 3011, 2928, 2836, 1593, 1496, $1446,1419,1326,1251,1219,1159,1112,1068,1030,1010,834,800,624,567 \mathrm{~cm}^{-1} ; \mathbf{R}_{\mathbf{f}}(8: 2$ hexanes/EtOAc; Vanillin): 0.41.<smiles>[Y4]C(OC(=S)c1ccc(OC)cc1)C(=O)OCC</smiles>

30 
Ethyl 2-((4-methoxyphenylcarbonothioyl)oxy)propanoate (3o). Prepared on 0.20-mmol scale following General Procedure A. The crude residue was purified by flash column chromatography (gradient of $0-70 \% \mathrm{PhMe} /$ hexanes) to yield the product as a yellow oil (30 $\mathrm{mg}, 0.11 \mathrm{mmol}$, 56\%). ${ }^{1} \mathbf{H}$ NMR $\left(500 \mathrm{MHz}, \mathrm{CDCl}_{3}, 298 \mathrm{~K}\right): \delta_{\mathrm{H}} 8.26-8.19(\mathrm{~m}, 2 \mathrm{H}), 6.91-6.84(\mathrm{~m}, 2 \mathrm{H}), 5.82(\mathrm{q}, J$ $=7.0 \mathrm{~Hz}, 1 \mathrm{H}), 4.26-4.20(\mathrm{~m}, 2 \mathrm{H}), 3.87(\mathrm{~s}, 3 \mathrm{H}), 1.72(\mathrm{~d}, J=7.0 \mathrm{~Hz}, 3 \mathrm{H}), 1.28(\mathrm{t}, J=7.1 \mathrm{~Hz}, 3 \mathrm{H})$ ppm. ${ }^{13} \mathbf{C}$ NMR $\left(126 \mathrm{MHz}, \mathrm{CDCl}_{3}, 298 \mathrm{~K}\right): \delta_{\mathrm{C}} 209.4,170.3,163.9,131.4,131.2,113.3,75.0$, 61.4, 55.5, 17.2, 14.1 ppm; HRMS $m / z$ (DART): calculated for $\mathrm{C}_{13} \mathrm{H}_{17} \mathrm{O}_{4} \mathrm{~S}(\mathrm{M}+\mathrm{H}): 269.0842$; found: 269.0848; IR (neat): 3025, 2931, 2858, 1738, 1451, 1354, 1277, 1253, 1181, 1084, 1009, 934, 89, 728, $559 \mathrm{~cm}^{-1} ; \mathbf{R}_{\mathbf{f}}$ (5:5 PhMe/hexanes; Vanillin): 0.20.

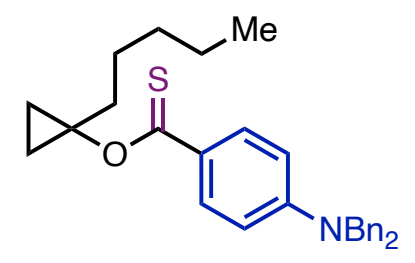

$3 p$

$O$-(1-pentylcyclopropyl) 4-(dibenzylamino)benzothioate (3p). Prepared on 0.20-mmol scale following General Procedure A. The crude residue was purified by flash column chromatography (gradient of $0-30 \% \mathrm{PhMe} /$ hexanes) to yield the product as a yellow oil $(86 \mathrm{mg}, 0.19 \mathrm{mmol}$, 97\%). ${ }^{1} \mathbf{H}$ NMR $\left(500 \mathrm{MHz}, \mathrm{CDCl}_{3}, 298 \mathrm{~K}\right): \delta_{\mathrm{H}} 8.08-8.01(\mathrm{~m}, 2 \mathrm{H}), 7.63-7.39(\mathrm{~m}, 4 \mathrm{H}), 7.31-7.25$ $(\mathrm{m}, 2 \mathrm{H}), 7.24-7.20(\mathrm{~m}, 4 \mathrm{H}), 6.71-6.64(\mathrm{~m}, 2 \mathrm{H}), 4.73(\mathrm{~s}, 4 \mathrm{H}), 2.11-2.02(\mathrm{~m}, 2 \mathrm{H}), 1.52-1.42(\mathrm{~m}$, 2H), 1.34-1.29 (m, 5H), 1.12-1.06 (m, 2H), 0.90-0.86 (m, 2H), 0.84-0.80 (m, 2H) ppm. ${ }^{13} \mathrm{C}$ NMR (126 MHz, $\left.\mathrm{CDCl}_{3}, 298 \mathrm{~K}\right): \delta_{\mathrm{C}} 208.8,152.7,137.3,131.1,129.0,128.4,127.4,126.6$, 111.2, 65.3, 54.3, 33.6, 31.8, 25.8, 22.8, 14.2, $13.2 \mathrm{ppm}$. HRMS $\mathrm{m} / z$ (DART): calculated for $\mathrm{C}_{29} \mathrm{H}_{34} \mathrm{NOS}(\mathrm{M}+\mathrm{H})$ : 444.2356; found: 444.2352; IR (neat): 3119, 3100, 2997, 2978, 2930, 2871, $1643,1513,1472,1436,1273,1229,1147,1089,1050,913,821,655,574 \mathrm{~cm}^{-1} ; \mathbf{R}_{\mathbf{f}}(8: 2$ hexanes:PhMe; Vanillin): 0.32 .

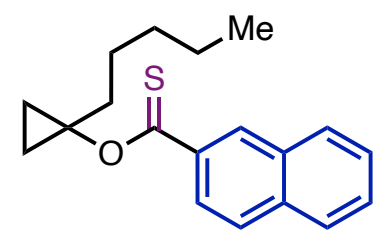

$3 q$

$\boldsymbol{O}$-(1-pentylcyclopropyl) naphthalene-2-carbothioate (3q). Prepared on 0.20-mmol scale following General Procedure A. The crude residue was purified by flash column chromatography (gradient of $0-30 \% \mathrm{PhMe} /$ hexanes) to yield the product as a yellow oil (49 $\mathrm{mg}, 0.17 \mathrm{mmol}$, 83\%). ${ }^{1} \mathbf{H}$ NMR $\left(500 \mathrm{MHz}, \mathrm{CDCl}_{3}, 298 \mathrm{~K}\right): \delta_{\mathrm{H}} 8.63(\mathrm{~d}, J=1.8 \mathrm{~Hz}, 1 \mathrm{H}), 8.22(\mathrm{dd}, J=8.7,1.9$ $\mathrm{Hz}, 1 \mathrm{H}), 7.96(\mathrm{ddt}, J=8.2,1.4,0.7 \mathrm{~Hz}, 1 \mathrm{H}), 7.84(\mathrm{dd}, J=8.2,1.2 \mathrm{~Hz}, 1 \mathrm{H}), 7.79$ (d, $J=8.8 \mathrm{~Hz}$, 1H), 7.60-7.48 (m, 2H), 2.13-2.07 (m, 2H), 1.57-1.47 (m, 2H), 1.36-1.25 (m, 4H), 1.22-1.17 (m, 2H), 0.95-0.81 (m, 5H) ppm. ${ }^{13} \mathbf{C}$ NMR (126 MHz, $\left.\mathrm{CDCl}_{3}, 298 \mathrm{~K}\right): \delta_{\mathrm{C}} 209.8,136.1,135.3$, 132.4, 129.8, 129.0, 128.2, 127.6, 127.6, 126.6, 125.4, 66.4, 33.1, 31.6, 25.7, 22.6, 14.0, 13.1 ppm. HRMS $m / z$ (DART): calculated for $\mathrm{C}_{19} \mathrm{H}_{23} \mathrm{OS}(\mathrm{M}+\mathrm{H}): 299.1464$; found: 299.1466; IR (neat): 3063, 3010, 2960, 2925, 2856, 1742, 1626, 1595, 1456, 1353, 1275, 1204, 1183, 1128, 1052, 1019, 946, 905, 861, 748, $625 \mathrm{~cm}^{-1}$; $\mathbf{R}_{\mathbf{f}}(9: 1$ hexanes/PhMe; Vanillin): 0.66. 
<smiles>CC(Cc1ccccc1)OC(=S)c1ccsc1</smiles>

$3 r$

$O$-(1-phenylpropan-2-yl) thiophene-3-carbothioate (3r). Prepared on 0.20-mmol scale following General Procedure A. The crude residue was purified by flash column chromatography (gradient of $0-20 \% \mathrm{PhMe} /$ hexanes) to yield the product as a yellow oil $(51 \mathrm{mg}, 0.19 \mathrm{mmol}$, 96\%). ${ }^{1} \mathbf{H}$ NMR $\left(400 \mathrm{MHz}, \mathrm{CDCl}_{3}, 298 \mathrm{~K}\right): \delta_{\mathrm{H}} 8.11(\mathrm{dd}, J=3.1,1.2 \mathrm{~Hz}, 1 \mathrm{H}), 7.68(\mathrm{dd}, J=5.1$, $1.2 \mathrm{~Hz}, 1 \mathrm{H}), 7.38-7.27(\mathrm{~m}, 5 \mathrm{H}), 7.26-7.24(\mathrm{~m}, 1 \mathrm{H}), 5.98(\mathrm{dtd}, J=6.9,6.2,5.8 \mathrm{~Hz}, 1 \mathrm{H}), 3.26$ (dd, $J=13.7,5.8 \mathrm{~Hz}, 1 \mathrm{H}), 2.99(\mathrm{dd}, J=13.7,6.9 \mathrm{~Hz}, 1 \mathrm{H}), 1.44(\mathrm{~d}, J=6.2 \mathrm{~Hz}, 3 \mathrm{H}) \mathrm{ppm} .{ }^{13} \mathbf{C} \mathbf{N M R}$ $\left(101 \mathrm{MHz}, \mathrm{CDCl}_{3}, 298 \mathrm{~K}\right): \delta_{\mathrm{C}} 204.3,143.7,137.3,130.4,129.7,128.7,128.6,126.8,125.4$, 78.5, 41.8, 18.7 ppm. HRMS $m / z$ (DART): calculated for $\mathrm{C}_{14} \mathrm{H}_{15} \mathrm{OS}_{2}(\mathrm{M}+\mathrm{H}): 263.0559$; found: 263.0566; IR (neat): 3023, 2944, 2935, 2936, 1729, 1607, 1509 1456, 1244, 1169, 1029, 819, $786 \mathrm{~cm}^{-1} ; \mathbf{R}_{\mathbf{f}}(7: 3$ hexanes/PhMe; Vanillin): 0.59.<smiles>Fc1ccc(C(=S)OCCCc2ccccc2)cc1</smiles>

3s

$\boldsymbol{O}$-(3-phenylpropyl) 4-fluorobenzothioate (3s). Prepared on 0.20-mmol scale following General Procedure A. The crude residue was purified by flash column chromatography (100\% hexanes) to yield the product as a yellow oil (48 mg, $0.17 \mathrm{mmol}, 85 \%) .{ }^{\mathbf{1}} \mathbf{H}$ NMR (400 $\mathrm{MHz}, \mathrm{CDCl}_{3}, 298$ $\mathrm{K}): \delta_{\mathrm{H}} 8.26-8.17(\mathrm{~m}, 2 \mathrm{H}), 7.39-7.32(\mathrm{~m}, 2 \mathrm{H}), 7.29-7.22(\mathrm{~m}, 3 \mathrm{H}), 7.16-7.06(\mathrm{~m}, 2 \mathrm{H}), 4.72(\mathrm{t}, J=$ $6.4 \mathrm{~Hz}, 2 \mathrm{H}), 2.93-2.85$ (m, 2H), 2.35-2.24 (m, 2H) ppm. ${ }^{13} \mathbf{C} \mathbf{N M R}\left(101 \mathrm{MHz}, \mathrm{CDCl}_{3}, 298 \mathrm{~K}\right)$ : $\delta_{\mathrm{C}} 209.9,166.0(\mathrm{~d}, J=254.8 \mathrm{~Hz}), 141.1,131.4(\mathrm{~d}, J=9.2 \mathrm{~Hz}), 128.7,128.7,128.6,126.3,115.3$ $(\mathrm{d}, J=22.0 \mathrm{~Hz}), 72.2,32.7,30.0 \mathrm{ppm} .{ }^{19} \mathbf{F} \mathbf{N M R}\left(377 \mathrm{MHz}, \mathrm{CDCl}_{3}, 298 \mathrm{~K}\right): \delta_{\mathrm{F}}-106.5 \mathrm{ppm}$; HRMS $m / z$ (DART): calculated for $\mathrm{C}_{16} \mathrm{H}_{16} \mathrm{FOS}(\mathrm{M}+\mathrm{H}): 275.0900$; found: 275.0892; IR (neat): 3067, 3026, 2936, 2923, 2864, 1724, 1595, 1500, 1453, 1412, 1304, 1267, 1225, 1154, 1098, 1051, 1012, 843, 744, 698, 630, $562 \mathrm{~cm}^{-1}$; $\mathbf{R}_{\mathbf{f}}$ (95:5 hexanes/PhMe; Vanillin): 0.91.<smiles>COc1ccc(C(=S)OCc2ccccc2)cc1OC</smiles>

3t

$\boldsymbol{O}$-benzyl 3,4-dimethoxybenzothioate (3t). Prepared on 0.20 -mmol scale following General Procedure A. The crude residue was purified by flash column chromatography (gradient of 0 $20 \% \mathrm{PhMe} /$ hexanes, then 5\% EtOAc/hexanes) to yield the product as a yellow solid (48 $\mathrm{mg}, 0.17$ mmol, 84\%). ${ }^{1} \mathbf{H}$ NMR (500 MHz, $\left.\mathrm{CDCl}_{3}, 298 \mathrm{~K}\right): \delta_{\mathrm{H}} 7.90-7.81(\mathrm{~m}, 2 \mathrm{H}), 7.51-7.33(\mathrm{~m}, 5 \mathrm{H})$, $6.83(\mathrm{~d}, J=8.5 \mathrm{~Hz}, 1 \mathrm{H}), 5.71(\mathrm{~s}, 2 \mathrm{H}), 3.94(\mathrm{~s}(\mathrm{br}), 6 \mathrm{H}) \mathrm{ppm} .{ }^{13} \mathbf{C} \mathbf{N M R}\left(126 \mathrm{MHz}, \mathrm{CDCl}_{3}, 298\right.$ 
$\mathrm{K}): \delta_{\mathrm{C}} 210.1,153.7,148.4,135.7,131.6,128.8,128.5,128.4,122.5,112.7,110.0,74.0,56.2$, $56.2 \mathrm{ppm}$. HRMS $\mathrm{m} / \mathrm{z}$ (DART): calculated for $\mathrm{C}_{16} \mathrm{H}_{17} \mathrm{O}_{3} \mathrm{~S}(\mathrm{M}+\mathrm{H}$ ): 289.0893; found: 289.0901; IR (neat): 3109, 3066, 3003, 2938, 2915, 2834, 1590, 1510, 1448, 1412, 1345, 1264, 1228, 1212 , $1165,1129,1009,983,804,726,692,612 \mathrm{~cm}^{-1} ; \mathbf{R}_{\mathbf{f}}$ (95:5 hexanes/PhMe; Vanillin): 0.40.<smiles>CC(Cc1ccccc1)OC(=S)c1ccc2c(ccn2C)c1</smiles>

\section{3u}

$O$-(1-phenylpropan-2-yl) 1-methyl-1H-indole-5-carbothioate (3u). Prepared on 0.20-mmol scale following General Procedure A. The crude residue was purified by flash column chromatography (gradient of $0-40 \% \mathrm{PhMe} /$ hexanes) to yield the product as a yellow solid (33 $\mathrm{mg}, 0.11 \mathrm{mmol}, 54 \%) .{ }^{1} \mathbf{H}$ NMR $\left(500 \mathrm{MHz}, \mathrm{CDCl}_{3}, 298 \mathrm{~K}\right): \delta_{\mathrm{H}} 8.53(\mathrm{dd}, J=1.9,0.6 \mathrm{~Hz}, 1 \mathrm{H})$, $8.14(\mathrm{dd}, J=8.8,1.7 \mathrm{~Hz}, 1 \mathrm{H}), 7.35-7.27(\mathrm{~m}, 4 \mathrm{H}), 7.26-7.21(\mathrm{~m}, 2 \mathrm{H}), 7.09(\mathrm{~d}, J=3.2 \mathrm{~Hz}, 1 \mathrm{H})$, $6.59(\mathrm{dd}, J=3.1,0.9 \mathrm{~Hz}, 1 \mathrm{H}), 6.12-6.06(\mathrm{~m}, 1 \mathrm{H}), 3.81(\mathrm{~s}, 3 \mathrm{H}), 3.31(\mathrm{dd}, J=13.6,5.7 \mathrm{~Hz}, 1 \mathrm{H})$, $3.01(\mathrm{dd}, J=13.6,7.1 \mathrm{~Hz}, 1 \mathrm{H}), 1.45(\mathrm{~d}, J=6.2 \mathrm{~Hz}, 3 \mathrm{H}) \mathrm{ppm} .{ }^{13} \mathbf{C}$ NMR $\left(126 \mathrm{MHz}, \mathrm{CDCl}_{3}, 298\right.$ K): $\delta_{\mathrm{C}} 211.6,139.3,137.5,131.2,130.3,129.6,128.4,127.8,126.5,123.5,123.2,108.4,103.3$, 78.5, 41.7, 33.1, $18.7 \mathrm{ppm}$. HRMS $\mathrm{m} / z$ (DART): calculated for $\mathrm{C}_{19} \mathrm{H}_{20} \mathrm{NOS}(\mathrm{M}+\mathrm{H}): 310.1260$; found: 310.1258; IR (neat): 3056, 3030, 2974, 2929, 2912, 1602, 1516, 1449, 1340, 1248, 1226, 1147, 1072, 1048, 811, 723, 701, $670 \mathrm{~cm}^{-1} ; \mathbf{R}_{\mathbf{f}}$ (7:3 hexanes/PhMe; Vanillin): 0.45.

Scheme S1. Unsuccessful scope entry attempts ${ }^{\mathrm{a}}$
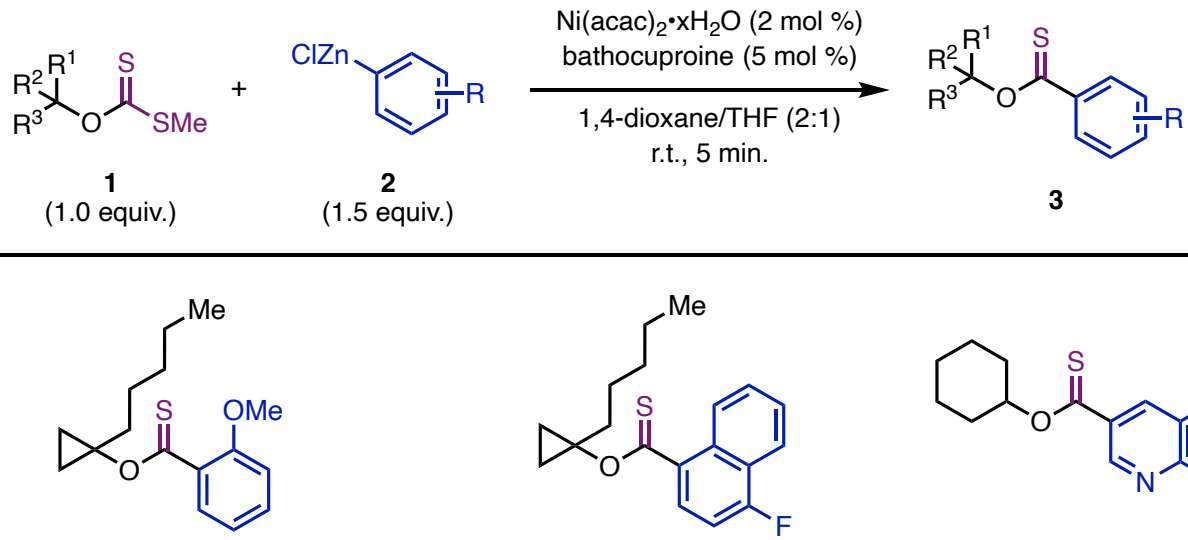<smiles>CCCCC1(OC(=S)c2ccc(F)c3ccccc23)CC1</smiles><smiles>S=C(OC1CCCCC1)c1cnc2ccccc2c1</smiles><smiles>COc1ccc(C(=S)OC2CN(C(=O)OC(C)(C)C)C2)cc1</smiles><smiles>COc1ccc(C(=S)OC(C(=O)c2ccccc2)c2ccccc2)cc1</smiles><smiles>COc1ccc(C(=S)Oc2ccccc2)cc1</smiles>

${ }^{\mathrm{a}}<20 \%$ uncalibrated GC-MS yield observed in all cases 


\section{Synthesis of aryl thioamides.}

\section{General procedure B: Preparation of aryl thioamides from thiocarbonyl imidazolides}
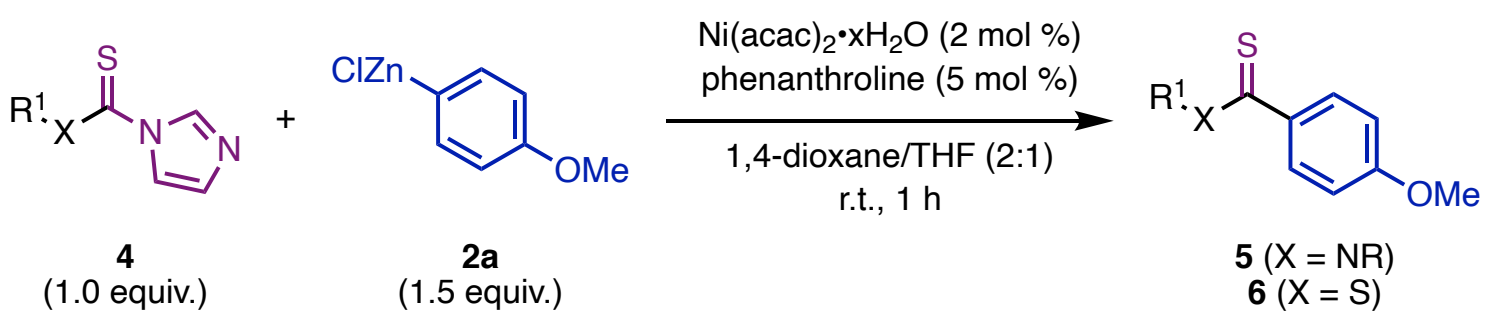

To a flame-dried 16-mL reaction tube with a stir bar was added thiocarbonyl imidazolide substrate ( $0.20 \mathrm{mmol}, 1.0$ equiv), $\mathrm{Ni}(\mathrm{acac})_{2} \bullet \mathrm{xH}_{2} \mathrm{O}(1.0 \mathrm{mg}, 0.0040 \mathrm{mmol}, 2.0 \mathrm{~mol} \%)$, and phenanthroline ( $1.8 \mathrm{mg}, 0.010 \mathrm{mmol}, 5.0 \mathrm{~mol} \%)$. The flask was sealed with a septum, and evacuated and backfilled with $\mathrm{N}_{2}(\times 3)$. 1,4-Dioxane (anhydrous, degassed for 15 minutes by sparging with argon; an amount to reach a 2:1 1,4-dioxane/THF ratio, according to the volume of titrated arylzinc(II) chloride reagent required for $0.30 \mathrm{mmol}$ ) was added and the reaction mixture was stirred at r.t. for $10 \mathrm{~min}$. After $10 \mathrm{~min}$, while stirring, arylzinc solution $(0.30 \mathrm{mmol}$ of a solution in THF (typically $0.86 \mathrm{~mL}$ of a $0.35 \mathrm{M}$ solution), 3.0 equiv.) was added at once and the reaction was stirred at r.t. $\left(23^{\circ} \mathrm{C}\right)$ for 1 hour. The reaction was opened to air and quenched with sat. aq. $\mathrm{NH}_{4} \mathrm{Cl}(\sim 1.5 \mathrm{~mL})$. If analyzing by GC-MS, $n$-dodecane was added as an internal standard. EtOAc $(\sim 2 \mathrm{~mL})$ was added and the reaction mixture was mixed vigorously through pipetting. The organic layer was filtered through a Pasteur pipette loaded with $\mathrm{MgSO}_{4}$ and celite. An additional $2 \mathrm{~mL}$ of EtOAc was added to the remaining aqueous layer, vigorously mixed, and passed through the same $\mathrm{MgSO}_{4} /$ celite plug. (The sulfur-containing aqueous waste was treated with bleach and disposed of appropriately.) The combined organic phase was concentrated, and the crude residue was purified by flash column chromatography to yield the desired thioamide product.<smiles>CCN(CC)C(=S)c1ccc(OC)cc1</smiles>

$5 a$

$\mathrm{N}, \mathrm{N}$-Diethyl-4-methoxybenzothioamide (5a). Prepared on 0.20-mmol scale following General Procedure B. The crude residue was purified by flash column chromatography (gradient of 0 $20 \% \mathrm{EtOAc} / \mathrm{hexanes}$ ) to yield the product as a yellow oil (44 $\mathrm{mg}, 0.20 \mathrm{mmol}, 98 \%) .{ }^{1} \mathbf{H}$ NMR $\left(500 \mathrm{MHz}, \mathrm{CDCl}_{3}, 298 \mathrm{~K}\right): \delta_{\mathrm{H}} 7.23-7.16(\mathrm{~m}, 2 \mathrm{H}), 6.88-6.82(\mathrm{~m}, 2 \mathrm{H}), 4.11(\mathrm{q}, J=7.1 \mathrm{~Hz}, 2 \mathrm{H})$, $3.80(\mathrm{~s}, 3 \mathrm{H}), 3.47$ (q, $J=7.1 \mathrm{~Hz}, 2 \mathrm{H}), 1.37(\mathrm{t}, J=7.1 \mathrm{~Hz}, 3 \mathrm{H}), 1.15(\mathrm{t}, J=7.2 \mathrm{~Hz}, 3 \mathrm{H}) \mathrm{ppm} .{ }^{13} \mathrm{C}$ NMR (126 MHz, $\left.\mathrm{CDCl}_{3}, 298 \mathrm{~K}\right): \delta_{\mathrm{C}} 200.6,159.6,136.7,126.9,113.7,55.5,47.9,46.4,14.0$, $11.4 \mathrm{ppm}$. HRMS $m / z$ (DART): calculated for $\mathrm{C}_{12} \mathrm{H}_{18} \mathrm{NOS}(\mathrm{M}+\mathrm{H}): 224.1104$; found: 224.1102; IR (neat): 3063, 2970, 2932, 2838, 1602, 1190, 1426, 1286, 1239, 1136, 1035, 920, 834, 768, $613 \mathrm{~cm}^{-1} ; \mathbf{R}_{\mathbf{f}}\left(8: 2 \mathrm{EtOAc} /\right.$ hexanes; $\left.\mathrm{KMnO}_{4}\right): 0.50$. 
<smiles>C=CCN(CC=C)C(=S)c1ccc(OC)cc1</smiles>

5b

$\mathrm{N}, \mathrm{N}$-Diallyl-4-methoxybenzothioamide (5b). Prepared on 0.20 -mmol scale following General Procedure B. The crude residue was purified by flash column chromatography (gradient of $0-$ $20 \%$ EtOAc/hexanes) to yield the product as a yellow oil (45 mg, $0.18 \mathrm{mmol}, 92 \%) .{ }^{1} \mathbf{H}$ NMR $\left(500 \mathrm{MHz}, \mathrm{CDCl}_{3}, 298 \mathrm{~K}\right): \delta_{\mathrm{H}} 7.29-7.26(\mathrm{~m}, 2 \mathrm{H}), 6.87-6.81(\mathrm{~m}, 2 \mathrm{H}), 6.01(\mathrm{ddt}, J=17.2,10.2$, $6.0 \mathrm{~Hz}, 1 \mathrm{H}), 5.72(\mathrm{ddt}, J=17.2,10.6,5.4 \mathrm{~Hz}, 1 \mathrm{H}), 5.34-5.16(\mathrm{~m}, 4 \mathrm{H}), 4.73(\mathrm{dt}, J=6.0,1.4 \mathrm{~Hz}$, $2 \mathrm{H}), 4.05(\mathrm{dt}, J=5.5,1.6 \mathrm{~Hz}, 2 \mathrm{H}), 3.81(\mathrm{~s}, 3 \mathrm{H}) \mathrm{ppm} .{ }^{13} \mathbf{C} \mathbf{N M R}\left(126 \mathrm{MHz}, \mathrm{CDCl}_{3}, 298 \mathrm{~K}\right) \delta_{\mathrm{C}}$ 202.7, 160.1, 136.1, 132.2, 131.0, 130.9, 127.4, 119.0, 113.7, 55.5, 55.1, $53.8 \mathrm{ppm}$. HRMS $\mathrm{m} / \mathrm{z}$ (DART): calculated for $\mathrm{C}_{14} \mathrm{H}_{18} \mathrm{NOS}(\mathrm{M}+\mathrm{H}): 248.1104$; found: 248.1108; IR (neat): 3088, 3006, 2930, 2836, 1641, 1604, 1511, 1466, 1405, 1297, 1244, 1218, 1173, 1028, 924, 828, 792, 670 $\mathrm{cm}^{-1} ; \mathbf{R}_{\mathbf{f}}\left(8: 2 \mathrm{EtOAc} /\right.$ hexanes; $\left.\mathrm{KMnO}_{4}\right): 0.59$.<smiles>COc1ccc(C(=S)N(Cc2ccccc2)Cc2ccccc2)cc1</smiles>

$5 c$

$\boldsymbol{N}, \boldsymbol{N}$-Dibenzyl-4-methoxybenzothioamide (5c). Prepared on 0.20 -mmol scale following General Procedure B. The crude residue was purified by flash column chromatography (gradient of $0-20 \%$ EtOAc/hexanes) to yield the product as a yellow oil (47 mg, $0.14 \mathrm{mmol}, 68 \%) .{ }^{1} \mathbf{H}$ NMR $\left(400 \mathrm{MHz}, \mathrm{CDCl}_{3}, 298 \mathrm{~K}\right) \delta_{\mathrm{H}} 7.40-7.31(\mathrm{~m}, 10 \mathrm{H}), 7.13-7.06(\mathrm{~m}, 2 \mathrm{H}), 6.88-6.82(\mathrm{~m}, 2 \mathrm{H})$, 5.39 (s, 2H), 4.66 (s, 2H), 3.79 (s, 3H) ppm. $\left.{ }^{13} \mathbf{C ~ N M R ~ ( 1 0 1 ~ M H z , ~ C D C l ~}, 298 \mathrm{~K}\right) \delta_{\mathrm{C}} 203.9$, 160.1, 136.1, 135.7, 135.5, 129.1, 129.0, 128.3, 128.1, 128.0, 127.5, 127.3, 113.9, 55.5, 55.5, $53.5 \mathrm{ppm}$. HRMS $\mathrm{m} / \mathrm{z}$ (DART): calculated for $\mathrm{C}_{22} \mathrm{H}_{22} \mathrm{NOS}(\mathrm{M}+\mathrm{H}): 348.1417$; found: 348.1419 ; IR (neat): 3067, 3029, 2929, 2839, 1733, 1603, 1509, 1468, 1451, 1296, 1245, 1173, 1028, 830, $739,698 \mathrm{~cm}^{-1} ; \mathbf{R}_{\mathbf{f}}\left(8: 2 \mathrm{EtOAc} /\right.$ hexanes; $\left.\mathrm{KMnO}_{4}\right): 0.55$.<smiles>COc1ccc(C(=S)N2CCCC2)cc1</smiles>

$5 d$

(4-Methoxyphenyl)(pyrrolidin-1-yl)methanethione (5d). Prepared on 0.20-mmol scale following General Procedure B. The crude residue was purified by flash column chromatography (gradient of 0-20\% EtOAc/hexanes) to yield the product as a yellow oil (39 $\mathrm{mg}, 0.18 \mathrm{mmol}$, 89\%). ${ }^{1} \mathbf{H}$ NMR $\left(500 \mathrm{MHz}, \mathrm{CDCl}_{3}, 298 \mathrm{~K}\right): \delta_{\mathrm{H}} 7.34-7.27(\mathrm{~m}, 2 \mathrm{H}), 6.82-6.76(\mathrm{~m}, 2 \mathrm{H}), 3.91(\mathrm{t}, J$ $=7.1 \mathrm{~Hz}, 2 \mathrm{H}), 3.75(\mathrm{~s}, 3 \mathrm{H}), 3.47(\mathrm{t}, J=6.8 \mathrm{~Hz}, 2 \mathrm{H}), 2.01(\mathrm{p}, J=6.9 \mathrm{~Hz}, 2 \mathrm{H}), 1.90(\mathrm{p}, J=6.7 \mathrm{~Hz}$, 2H) ppm; ${ }^{13} \mathbf{C}$ NMR (126 MHz, $\left.\mathrm{CDCl}_{3}, 298 \mathrm{~K}\right): \delta_{\mathrm{C}} 197.3,160.2,136.6,127.9,113.6,55.5,54.1$, 53.9, 26.7, 24.8 ppm; HRMS $m / z$ (DART): calculated for $\mathrm{C}_{12} \mathrm{H}_{16} \mathrm{NOS}(\mathrm{M}+\mathrm{H}): 222.0947$; found: 
222.0939; IR (neat): 2974, 2952, 2867, 1742, 1605, 1511, 1470, 1437, 1300, 1252, 1171, 1031, 955, 827, 812, 785, $575 \mathrm{~cm}^{-1} ; \mathbf{R}_{\mathbf{f}}\left(8: 2\right.$ hexanes/EtOAc; $\left.\mathrm{KMnO}_{4}\right): 0.35$.<smiles>COc1ccc(C(=S)N2CCOCC2)cc1</smiles>

$5 e$

(4-Methoxyphenyl)(morpholino)methanethione (5e). Prepared on 0.20-mmol scale following General Procedure B. The crude residue was purified by flash column chromatography (gradient of 0-30\% EtOAc/hexanes) to yield the product as a yellow solid (47 mg, $0.20 \mathrm{mmol}, 98 \%) .{ }^{1} \mathbf{H}$ NMR (500 MHz, $\mathrm{CDCl}_{3}, 298 \mathrm{~K}$ ): $\delta_{\mathrm{H}} 7.31-7.26(\mathrm{~m}, 2 \mathrm{H}), 6.90-6.84(\mathrm{~m}, 2 \mathrm{H}), 4.42(\mathrm{~s}(\mathrm{br}), 2 \mathrm{H})$, 3.88 (s (br), 2H), $3.82(\mathrm{~s}, 3 \mathrm{H}), 3.75-3.57(\mathrm{~m}, 4 \mathrm{H}) \mathrm{ppm} .{ }^{13} \mathbf{C}$ NMR $\left(126 \mathrm{MHz}, \mathrm{CDCl}_{3}, 298 \mathrm{~K}\right): \delta_{\mathrm{C}}$ 201.4, 160.5, 135.1, 128.2, 113.9, 66.9, 66.7, 55.6, 52.9, $50.2 \mathrm{ppm}$. HRMS $m / z$ (DART):

calculated for $\mathrm{C}_{12} \mathrm{H}_{16} \mathrm{NO}_{2} \mathrm{~S}(\mathrm{M}+\mathrm{H})$ : 238.0896; found: 238.0895; IR (neat): 2996, 2924, 2838 , $1608,1573,1511,1479,1429,1284,1258,1227,1169,1111,1024,875,815,633,594 \mathrm{~cm}^{-1} ; \mathbf{R}_{\mathbf{f}}$ (8:2 hexanes/EtOAc; $\left.\mathrm{KMnO}_{4}\right)$ : 0.20 .<smiles>COc1ccc(C(=S)N2CCC(C#N)CC2)cc1</smiles>

$5 f$

1-(4-Methoxyphenylcarbonothioyl)piperidine-4-carbonitrile (5f). Prepared on 0.20-mmol scale following General Procedure B. The crude residue was purified by flash column chromatography (gradient of 0-20\% EtOAc/hexanes) to yield the product as a yellow oil ( $45 \mathrm{mg}$, $0.17 \mathrm{mmol}, 86 \%) .{ }^{1} \mathbf{H}$ NMR $\left(400 \mathrm{MHz}, \mathrm{CDCl}_{3}, 298 \mathrm{~K}\right): \delta_{\mathrm{H}} 7.33-7.24(\mathrm{~m}, 2 \mathrm{H}), 6.92-6.85(\mathrm{~m}$, 2H), 4.47 (s (br), 2H), 3.84 (s (br), 4H), 3.67 (s (br), 1H), 3.02 (tt, $J=7.0,4.5 \mathrm{~Hz}, 1 \mathrm{H}), 2.12$ (s (br), 2H), 1.89 (s (br), 2H) ppm. ${ }^{13} \mathbf{C}$ NMR (101 MHz, CDCl $\left.3,298 \mathrm{~K}\right): \delta_{\mathrm{C}} 201.9,160.5,135.2$, 127.9, 120.5, 113.9, 55.5, 50.0, 47.6, 29.5, 28.1, $26.2 \mathrm{ppm}$. HRMS $\mathrm{m} / z$ (DART): calculated for $\mathrm{C}_{14} \mathrm{H}_{17} \mathrm{~N}_{2} \mathrm{OS}(\mathrm{M}+\mathrm{H}): 261.1056$; found: 261.1055; IR (neat): 3022, 2951, 2922, 2850, 2264 , 1603, 1570, 1481, 1440, 1296, 1248, 1193, 1173, 997, 817, $656 \mathrm{~cm}^{-1} ; \mathbf{R}_{\mathbf{f}}(8: 2$ hexanes/EtOAc; $\left.\mathrm{KMnO}_{4}\right): 0.23$.<smiles>COc1ccc(C(=S)N2CCC3(CC2)OCCO3)cc1</smiles>

$5 \mathrm{~g}$

(4-Methoxyphenyl)(1,4-dioxa-8-azaspiro[4.5]decan-8-yl)methanethione (5g). Prepared on $0.20-\mathrm{mmol}$ scale following General Procedure B. The crude residue was purified by flash column chromatography (gradient of $0-30 \%$ EtOAc/hexanes) to yield the product as a paleyellow solid (40 mg, $0.14 \mathrm{mmol}, 70 \%) .{ }^{1} \mathbf{H}$ NMR $\left(500 \mathrm{MHz}, \mathrm{CDCl}_{3}, 298 \mathrm{~K}\right)$ : $\delta_{\mathrm{H}} 7.50-7.48(\mathrm{~m}$, $2 \mathrm{H}), 7.09-7.06(\mathrm{~m}, 2 \mathrm{H}), 4.69(\mathrm{t}, J=6.0 \mathrm{~Hz}, 2 \mathrm{H}), 4.26-4.17(\mathrm{~m}, 4 \mathrm{H}), 4.03(\mathrm{~s}, 3 \mathrm{H}), 3.96-3.89(\mathrm{~m}$, 
2H), 2.15 (t, $J=5.9 \mathrm{~Hz}, 2 \mathrm{H}), 1.90(\mathrm{t}, J=5.9 \mathrm{~Hz}, 2 \mathrm{H}) \mathrm{ppm} .{ }^{13} \mathbf{C} \mathbf{N M R}\left(126 \mathrm{MHz}, \mathrm{CDCl}_{3}, 298 \mathrm{~K}\right)$ : $\delta_{\mathrm{C}} 201.0,160.3,135.8,127.8,113.8,106.6,64.8,55.5,50.2,48.1,36.0,34.6 \mathrm{ppm}$. HRMS $\mathrm{m} / \mathrm{z}$ (DART): calculated for $\mathrm{C}_{15} \mathrm{H}_{20} \mathrm{NO}_{3} \mathrm{~S}(\mathrm{M}+\mathrm{H})$ : 294.1158; found: 294.1160; IR (neat): 3074, 2999, 2958, 2989, 2836, 1746, 1605, 1486, 1431, 1290, 1235, 1090, 1029, 938, 912, 808, 628, 577 $\mathrm{cm}^{-1} ; \mathbf{R}_{\mathbf{f}}\left(8: 2\right.$ hexanes/EtOAc; $\left.\mathrm{KMnO}_{4}\right): 0.20$.

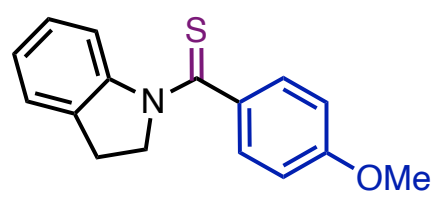

$5 \mathrm{~h}$

Indolin-1-yl(4-methoxyphenyl)methanethione (5h). Prepared on 0.20-mmol scale following General Procedure B. The crude residue was purified by flash column chromatography (gradient of $0-20 \%$ EtOAc/hexanes) to yield the product as a yellow oil (47 $\mathrm{mg}, 0.17 \mathrm{mmol}, 87 \%) .{ }^{1} \mathbf{H}$ NMR (500 MHz, $\left.\mathrm{CDCl}_{3}, 298 \mathrm{~K}\right): \delta_{\mathrm{H}} 7.42-7.39(\mathrm{~m}, 2 \mathrm{H}), 7.27-7.23(\mathrm{~m}, 2 \mathrm{H}), 6.97(\mathrm{~s}, 1 \mathrm{H}), 6.91-$ $6.83(\mathrm{~m}, 3 \mathrm{H}), 4.59$ (s (br), 2H), $3.86(\mathrm{~s}, 3 \mathrm{H}), 3.19$ (t, $J=7.9 \mathrm{~Hz}, 2 \mathrm{H}) \mathrm{ppm} .{ }^{13} \mathbf{C}$ NMR $(126 \mathrm{MHz}$, $\left.\mathrm{CDCl}_{3}, 298 \mathrm{~K}\right) \delta_{\mathrm{C}} 197.2,161.0,142.9,135.8,135.5,128.8,126.8,125.9,124.6,117.3,113.9$, 57.3, 55.5, $27.1 \mathrm{ppm}$. HRMS $\mathrm{m} / z$ (DART): calculated for $\mathrm{C}_{16} \mathrm{H}_{16} \mathrm{NOS}(\mathrm{M}+\mathrm{H}): 270.0947$; found: 270.0954; IR (neat): 3013, 2964, 2933, 2837, 2028, 1953, 1735, 1605, 1508, 1479, 1398, 1331, $1249,1166,955,825,802,750,576 \mathrm{~cm}^{-1} ; \mathbf{R}_{\mathbf{f}}\left(8: 2\right.$ hexanes/EtOAc; $\left.\mathrm{KMnO}_{4}\right): 0.54$.<smiles>COc1ccc(C(=S)N2CCCc3ccccc32)cc1</smiles>

$5 i$

(3,4-dihydroquinolin-1(2H)-yl)(4-methoxyphenyl)methanethione (5i). Prepared on 0.20mmol scale following General Procedure B. The crude residue was purified by flash column chromatography (gradient of 0-20\% EtOAc/hexanes) to yield the product as a yellow oil (46 mg, $0.16 \mathrm{mmol}, 81 \%$ ). ${ }^{1} \mathbf{H}$ NMR $\left(500 \mathrm{MHz}, \mathrm{CDCl}_{3}, 298 \mathrm{~K}\right): \delta_{\mathrm{H}} 7.25-7.20(\mathrm{~m}, 2 \mathrm{H}), 7.16$ (dd, $J=7.6$, $1.4 \mathrm{~Hz}, 1 \mathrm{H}), 7.01(\mathrm{td}, J=7.5,1.2 \mathrm{~Hz}, 1 \mathrm{H}), 6.88-6.79(\mathrm{~m}, 1 \mathrm{H}), 6.68-6.61(\mathrm{~m}, 2 \mathrm{H}), 6.58-6.53(\mathrm{~m}$, $1 \mathrm{H}), 4.42(\mathrm{t}, J=6.8 \mathrm{~Hz}, 2 \mathrm{H}), 3.75(\mathrm{~s}, 3 \mathrm{H}), 2.83(\mathrm{t}, J=6.5 \mathrm{~Hz}, 2 \mathrm{H}), 2.15(\mathrm{p}, J=6.7 \mathrm{~Hz}, 2 \mathrm{H}) \mathrm{ppm}$. ${ }^{13}$ C NMR (126 MHz, $\left.\mathrm{CDCl}_{3}, 298 \mathrm{~K}\right): \delta_{\mathrm{C}} 201.4,160.6,141.6,136.6,133.5,130.4,128.0,126.4$, 126.2, 125.8, 113.1, 55.4, 52.2, 27.1, 24.9 ppm. HRMS $m / z$ (DART): calculated for $\mathrm{C}_{17} \mathrm{H}_{18} \mathrm{NOS}$ (M+H): 284.1104; found: 284.1112; IR (neat): 3065, 2945, 2832, 1599, 1572, 1485, 1370, 1352, 1247, 1169, 1090, 821, 973, 834, 755, $577 \mathrm{~cm}^{-1} ; \mathbf{R}_{\mathbf{f}}\left(8: 2\right.$ hexanes/EtOAc; $\left.\mathrm{KMnO}_{4}\right): 0.57$.<smiles>COc1ccc(C(=S)N2CCCCCC2)cc1</smiles>

$5 \mathbf{j}$ 
Azepan-1-yl(4-methoxyphenyl)methanethione (5j). Prepared on 0.20-mmol scale following General Procedure B. The crude residue was purified by flash column chromatography (gradient of 0-20\% EtOAc/hexanes) to yield the product as a yellow oil (47 mg, $0.19 \mathrm{mmol}, 94 \%) .{ }^{1} \mathbf{H}$ NMR $\left(400 \mathrm{MHz}, \mathrm{CDCl}_{3}, 298 \mathrm{~K}\right) \delta_{\mathrm{H}} 7.27-7.19(\mathrm{~m}, 2 \mathrm{H}), 6.93-6.83(\mathrm{~m}, 2 \mathrm{H}), 4.27-4.19(\mathrm{~m}, 2 \mathrm{H})$, $3.83(\mathrm{~s}, 3 \mathrm{H}), 3.63(\mathrm{t}, J=5.7 \mathrm{~Hz}, 2 \mathrm{H}), 2.06-2.00(\mathrm{~m}, 2 \mathrm{H}), 1.73-1.58(\mathrm{~m}, 6 \mathrm{H}) \mathrm{ppm} .{ }^{13} \mathbf{C} \mathbf{N M R}$ $\left(101 \mathrm{MHz}, \mathrm{CDCl}_{3}, 298 \mathrm{~K}\right) \delta_{\mathrm{C}} 200.6,159.5,136.7,127.0,113.6,55.4,54.5,54.0,29.1,27.5$, 26.2, $25.5 \mathrm{ppm}$. HRMS $\mathrm{m} / z$ (DART): calculated for $\mathrm{C}_{14} \mathrm{H}_{20} \mathrm{NOS}(\mathrm{M}+\mathrm{H}): 250.1260$; found: 250.1270; IR (neat): 3009, 2967, 2939, 2855, 1605, 1493, 1420, 1300, 1239, 1196, 1170, 1026, $905,815,631,564 \mathrm{~cm}^{-1} ; \mathbf{R}_{\mathbf{f}}\left(8: 2\right.$ hexanes/EtOAc; $\left.\mathrm{KMnO}_{4}\right): 0.27$.<smiles>CCCSC(=S)c1ccc(OC)cc1</smiles>

$6 a$

Propyl 4-methoxybenzodithioate (6a). Prepared on 0.20-mmol scale following General Procedure B. The crude residue was purified by flash column chromatography (gradient of 0 $20 \% \mathrm{PhMe} /$ hexanes $)$ to yield the product as a red oil $(13 \mathrm{mg}, 0.06 \mathrm{mmol}, 32 \%) .{ }^{1} \mathbf{H}$ NMR (400 $\left.\mathrm{MHz}, \mathrm{CDCl}_{3}, 298 \mathrm{~K}\right) \delta_{\mathrm{H}} 8.12-8.04(\mathrm{~m}, 2 \mathrm{H}), 6.89-6.85(\mathrm{~m}, 2 \mathrm{H}), 3.86(\mathrm{~s}, 3 \mathrm{H}), 3.38-3.33(\mathrm{~m}, 2 \mathrm{H})$, $1.80(\mathrm{sxt}, J=7.4 \mathrm{~Hz}, 2 \mathrm{H}), 1.08(\mathrm{t}, J=7.4 \mathrm{~Hz}, 3 \mathrm{H}) \mathrm{ppm} .{ }^{13} \mathbf{C} \mathbf{N M R}\left(101 \mathrm{MHz}, \mathrm{CDCl}_{3}, 298 \mathrm{~K}\right) \delta_{\mathrm{C}}$ 226.5, 163.6, 138.5, 129.1, 113.5, 55.7, 39.1, 21.2, 13.9 ppm. HRMS $m / z$ (DART): calculated for $\mathrm{C}_{11} \mathrm{H}_{15} \mathrm{OS}_{2}(\mathrm{M}+\mathrm{H})$ : 227.0559; found: 227.0556; IR (neat): 2960, 2927, 2871, 2839, 2045, 1594, 1501, 1457, 1307, 1237, 1168, 1034, 884, 832, 633, $590 \mathrm{~cm}^{-1} ; \mathbf{R}_{\mathbf{f}}(9: 1$ hexanes/PhMe; $\left.\mathrm{KMnO}_{4}\right): 0.31$. 


\section{E. Synthesis of alkyl thionoesters and thioamides}

\section{General procedure C: Synthesis of alkyl thionoesters and thioamides from thiocarbonyl imidazolides}

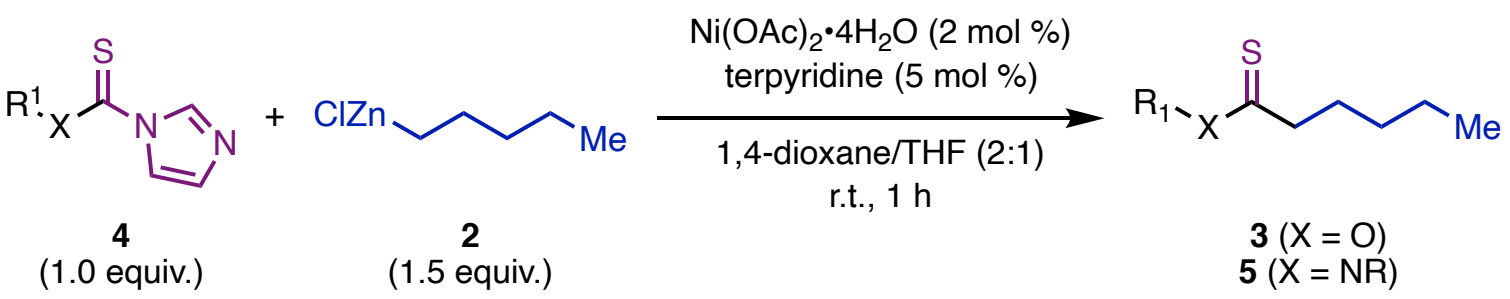

To a flame-dried $16-\mathrm{mL}$ reaction tube with a stir bar was added thiocarbonyl imidazolide substrate ( $0.20 \mathrm{mmol}, 1.0$ equiv), $\mathrm{Ni}(\mathrm{acac})_{2} \bullet \mathrm{xH}_{2} \mathrm{O}(1.0 \mathrm{mg}, 0.020 \mathrm{mmol}, 2.0 \mathrm{~mol} \%)$, and $2,2^{\prime}: 6^{\prime}, 2^{\prime \prime}$-Terpyridine ( $\left.2.3 \mathrm{mg}, 0.050 \mathrm{mmol}, 5.0 \mathrm{~mol} \%\right)$. The flask was sealed with a septum, and evacuated and backfilled with $\mathrm{N}_{2}(\times 3)$. 1,4-Dioxane (anhydrous, degassed for 15 minutes by sparging with argon; an amount to reach a 2:1 1,4-dioxane/THF ratio, according to the volume of titrated alkylzinc(II) chloride reagent required for $0.30 \mathrm{mmol}$ ) was added and the reaction mixture was stirred at r.t. for $10 \mathrm{~min}$. After $10 \mathrm{~min}$, while stirring, alkylzinc solution $(0.30 \mathrm{mmol}$ of a solution in THF (typically $0.86 \mathrm{~mL}$ of a $0.35 \mathrm{M}$ solution), 3.0 equiv.) was added at once and the reaction was stirred at r.t. $\left(23^{\circ} \mathrm{C}\right)$ for 1 hour. The reaction was opened to air and quenched with sat. aq. $\mathrm{NH}_{4} \mathrm{Cl}(\sim 1.5 \mathrm{~mL})$. If analyzing by GC-MS, $n$-dodecane was added as an internal standard. EtOAc $(\sim 2 \mathrm{~mL})$ was added and the reaction mixture was mixed vigorously through pipetting. The organic layer was filtered through a Pasteur pipette loaded with $\mathrm{MgSO}_{4}$ and celite. An additional $2 \mathrm{~mL}$ of EtOAc was added to the remaining aqueous layer, vigorously mixed, and passed through the same $\mathrm{MgSO}_{4} /$ celite plug. (The sulfur-containing aqueous waste was treated with bleach and disposed of appropriately.) The combined organic phase was concentrated, and the crude residue was purified by flash column chromatography to yield the desired thioamide product.<smiles>CCCCCC(=S)OCCCc1ccccc1</smiles>

3v

$\boldsymbol{O}$-(3-phenylpropyl) hexanethioate (3v). Prepared on 0.20 -mmol scale following General Procedure $\mathrm{C}$. The crude residue was purified by flash column chromatography (gradient of 0 $10 \% \mathrm{PhMe} /$ hexanes) to yield the product as a yellow oil (50 mg, $0.12 \mathrm{mmol}, 60 \%) .{ }^{1} \mathbf{H}$ NMR $\left(400 \mathrm{MHz}, \mathrm{CDCl}_{3}, 298 \mathrm{~K}\right) \delta_{\mathrm{H}} 7.34-7.27(\mathrm{~m}, 2 \mathrm{H}), 7.24-7.15(\mathrm{~m}, 3 \mathrm{H}), 4.47(\mathrm{t}, J=6.4 \mathrm{~Hz}, 2 \mathrm{H})$, 2.77-2.72 (m, 4H), 2.16-2.07 (m, 2H), 1.81-1.69 (m, 2H), 1.42-1.30 (m, 4H), 1.00-0.85 (m, $3 \mathrm{H})$ ppm. ${ }^{13} \mathbf{C}$ NMR $\left(101 \mathrm{MHz}, \mathrm{CDCl}_{3}, 298 \mathrm{~K}\right) \delta_{\mathrm{C}} 224.8,141.2,128.6,128.5,126.2,71.6,47.2$, 32.4, 31.2, 29.9, 28.5, 22.5, $14.1 \mathrm{ppm}$. HRMS $m / z$ (DART): calculated for $\mathrm{C}_{15} \mathrm{H}_{23} \mathrm{OS}(\mathrm{M}+\mathrm{H})$ : 251.1464; found: 251.1476; IR (neat): 3067, 3031, 2960, 2931, 2861, 1721, 1607, 1496, 1453, 1358, 1287, 1253, 1176, 1087, 1014, 743, 697, $584 \mathrm{~cm}^{-1}$; $\mathbf{R}_{\mathbf{f}}(9: 1$ hexanes/EtOAc; Vanillin): 0.34 . 


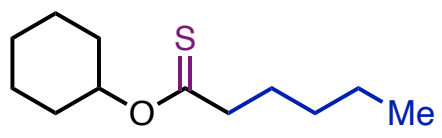

3w

$\boldsymbol{O}$-cyclohexyl hexanethioate (3w). Prepared on 0.20-mmol scale following General Procedure C. The crude residue was purified by flash column chromatography (gradient of $0-5 \%$ $\mathrm{PhMe} / \mathrm{hexanes})$ to yield the product as a yellow oil $(28 \mathrm{mg}, 0.13 \mathrm{mmol}, 64 \%) .{ }^{1} \mathbf{H}$ NMR (500 $\left.\mathrm{MHz}, \mathrm{CDCl}_{3}, 298 \mathrm{~K}\right) \delta_{\mathrm{H}} 5.43(\mathrm{tt}, J=8.9,3.9 \mathrm{~Hz}, 1 \mathrm{H}), 2.73-2.66(\mathrm{~m}, 2 \mathrm{H}), 1.98-1.89(\mathrm{~m}, 2 \mathrm{H})$, $1.77-1.66(\mathrm{~m}, 4 \mathrm{H}), 1.60-1.51(\mathrm{~m}, 3 \mathrm{H}), 1.46-1.38(\mathrm{~m}, 2 \mathrm{H}), 1.37-1.27(\mathrm{~m}, 5 \mathrm{H}), 0.94-0.84(\mathrm{~m}$, $3 \mathrm{H})$ ppm. ${ }^{13} \mathrm{C}$ NMR (126 MHz, $\left.\mathrm{CDCl}_{3}, 298 \mathrm{~K}\right) \delta_{\mathrm{C}} 224.0,80.0,47.9,31.1,30.8,28.5,25.5,23.7$, 22.5, $14.1 \mathrm{ppm}$. HRMS $m / z$ (DART): calculated for $\mathrm{C}_{12} \mathrm{H}_{23} \mathrm{OS}(\mathrm{M}+\mathrm{H}): 215.1464$; found: 215.1472; IR (neat): 3063, 3035, 2974, 2931, 2914, 1746, 1600, 1448, 1340, 1296, 1253, 1224, 1200, 1170, 1148, 1101, 1073, 1049, 927, 810, 723, 701, 598, 571 cm $\mathrm{cm}^{-1} \mathbf{R}_{\mathbf{f}}(9: 1$ hexanes/PhMe; Vanillin): 0.72 .<smiles>CCCCCC(=S)OC(C)C(=O)OC</smiles>

$3 x$

Methyl 2-(hexanethioyloxy)propanoate (3x). Prepared on $0.20-\mathrm{mmol}$ scale following General Procedure $\mathrm{C}$. The crude residue was purified by flash column chromatography (gradient of 0 $20 \% \mathrm{PhMe} / \mathrm{hexanes})$ to yield the product as a yellow oil (19 $\mathrm{mg}, 0.08 \mathrm{mmol}, 44 \%) .{ }^{\mathbf{1}} \mathbf{H} \mathbf{~ N M R}$ $\left(500 \mathrm{MHz}, \mathrm{CDCl}_{3}, 298 \mathrm{~K}\right) \delta_{\mathrm{H}} 5.63(\mathrm{q}, J=7.1 \mathrm{~Hz}, 1 \mathrm{H}), 3.74(\mathrm{~s}, 3 \mathrm{H}), 2.81-2.74(\mathrm{~m}, 2 \mathrm{H}), 1.83-$ $1.73(\mathrm{~m}, 2 \mathrm{H}), 1.60(\mathrm{~d}, J=7.0 \mathrm{~Hz}, 3 \mathrm{H}), 1.37-1.31(\mathrm{~m}, 4 \mathrm{H}), 0.92-0.88(\mathrm{~m}, 3 \mathrm{H}) .{ }^{13} \mathbf{C}$ NMR $(126$ $\left.\mathrm{MHz}_{2} \mathrm{CDCl}_{3}, 298 \mathrm{~K}\right) \delta_{\mathrm{C}} 224.0,170.6,74.9,52.5,46.8,31.1,28.5,22.5,17.2,14.1 \mathrm{ppm}$. HRMS $m / z$ (DART): calculated for $\mathrm{C}_{10} \mathrm{H}_{19} \mathrm{O}_{3} \mathrm{~S}(\mathrm{M}+\mathrm{H}): 219.1049$; found: 219.1045; IR (neat): 3067 , 3035, 2983, 2929, 2838, 1498, 1574, 1504, 1451, 1420, 1323, 1253, 1214, 1165, 1111, 1024, 837, 758, $635 \mathrm{~cm}^{-1}$; $\mathbf{R}_{\mathbf{f}}$ (7:3 hexanes/PhMe; Vanillin): 0.41.<smiles>C=CCN(CC=C)C(=S)CCCCC</smiles>

$5 \mathrm{k}$

$\mathbf{N}, \mathbf{N}$-diallylhexanethioamide (5k). Prepared on 0.20 -mmol scale following General Procedure $\mathrm{C}$. The crude residue was purified by flash column chromatography (gradient of $0-5 \%$ EtOAc/hexanes) to yield the product as a yellow oil (38 mg, $0.18 \mathrm{mmol}, 90 \%) .{ }^{1} \mathbf{H}$ NMR (400 $\left.\mathrm{MHz}, \mathrm{CDCl}_{3}, 298 \mathrm{~K}\right): \delta_{\mathrm{H}} 5.98-5.75(\mathrm{~m}, 2 \mathrm{H}), 5.33-5.23(\mathrm{~m}, 2 \mathrm{H}), 5.20(\mathrm{ddd}, J=17.7,2.7,1.5 \mathrm{~Hz}$, $2 \mathrm{H}), 4.66(\mathrm{dt}, J=6.0,1.5 \mathrm{~Hz}, 2 \mathrm{H}), 4.16(\mathrm{dt}, J=4.7,1.8 \mathrm{~Hz}, 2 \mathrm{H}), 2.82-2.76(\mathrm{~m}, 2 \mathrm{H}), 1.83-1.76$ $(\mathrm{m}, 2 \mathrm{H}), 1.40-1.34(\mathrm{~m} 4 \mathrm{H}), 0.94-0.90(\mathrm{~m}, 3 \mathrm{H}) \mathrm{ppm} .{ }^{13} \mathbf{C} \mathbf{N M R}\left(101 \mathrm{MHz}, \mathrm{CDCl}_{3}, 298 \mathrm{~K}\right): \delta_{\mathrm{C}}$ 206.0, 131.3 131.1, 118.4, 118.0, 55.6, 52.9, 43.4, 31.7, 29.9, 22.5, $14.1 \mathrm{ppm}$. HRMS m/z (DART): calculated for $\mathrm{C}_{12} \mathrm{H}_{22} \mathrm{NS}(\mathrm{M}+\mathrm{H}): 212.1468$; found: 212.1456; IR (neat): 3088, 2956, 
$2925,2863,1642,1483,1467,1407,1339,1295,1241,1207,1080,923,685,566 \mathrm{~cm}^{-1} ; \mathbf{R}_{\mathbf{f}}(8: 2$ hexanes/EtOAc; $\mathrm{KMnO}_{4}$ ): 0.77.<smiles>CCCCC(=S)N1CCOCC1</smiles>

51

1-Morpholinohexane-1-thione (5l). Prepared on 0.20-mmol scale following General Procedure C. The crude residue was purified by flash column chromatography (gradient of $0-20 \%$ EtOAc/hexanes) to yield the product as a white solid (30 mg, $0.15 \mathrm{mmol}, 75 \%) .{ }^{1} \mathbf{H}$ NMR (400 $\left.\mathrm{MHz}, \mathrm{CDCl}_{3}, 298 \mathrm{~K}\right): \delta_{\mathrm{H}} 4.37(\mathrm{t}, J=4.9 \mathrm{~Hz}, 2 \mathrm{H}), 3.82-3.78(\mathrm{~m}, 2 \mathrm{H}), 3.77(\mathrm{~s}(\mathrm{br}), 4 \mathrm{H}), 2.93-$ $2.84(\mathrm{~m}, 2 \mathrm{H}), 1.76-1.67(\mathrm{~m}, 2 \mathrm{H}), 1.44-1.34(\mathrm{~m}, 4 \mathrm{H}), 0.98-0.89(\mathrm{~m}, 3 \mathrm{H}) \mathrm{ppm} .{ }^{13} \mathbf{C}$ NMR $(101$ $\left.\mathrm{MHz}, \mathrm{CDCl}_{3}, 298 \mathrm{~K}\right): \delta_{\mathrm{C}} 204.2,66.7,66.7,50.2,50.1,43.8,31.7,29.1,22.5,14.1 \mathrm{ppm}$. HRMS $m / z$ (DART): calculated for $\mathrm{C}_{10} \mathrm{H}_{20} \mathrm{NOS}(\mathrm{M}+\mathrm{H}): 202.1260$; found: 202.1259; IR (neat): 2960, 2919, 2851, 1738, 1468, 1430, 1377, 1274, 1239, 1114, 1086, 1023, 972, 881, 837, 723, 579 $\mathrm{cm}^{-1} ; \mathbf{R}_{\mathbf{f}}\left(8: 2\right.$ hexanes/EtOAc; $\left.\mathrm{KMnO}_{4}\right): 0.54$.<smiles>CCCCCC(=S)N1CCCC1</smiles>

$5 \mathrm{~m}$

1-(pyrrolidin-1-yl)hexane-1-thione (5m). Prepared on 0.20-mmol scale following General Procedure $\mathrm{C}$. The crude residue was purified by flash column chromatography (gradient of 0 $20 \%$ EtOAc/hexanes) to yield the product as a yellow oil (18 mg, $0.10 \mathrm{mmol}, 48 \%) .{ }^{1} \mathbf{H ~ N M R}$ $\left(400 \mathrm{MHz}, \mathrm{CDCl}_{3}, 298 \mathrm{~K}\right) \delta_{\mathrm{H}} 3.82(\mathrm{t}, J=7.0 \mathrm{~Hz}, 2 \mathrm{H}), 3.60(\mathrm{t}, J=6.8 \mathrm{~Hz}, 2 \mathrm{H}), 2.70-2.62(\mathrm{~m}$, $2 \mathrm{H}), 2.08-2.01(\mathrm{~m}, 2 \mathrm{H}), 2.00-1.90(\mathrm{~m}, 2 \mathrm{H}), 1.79-1.69(\mathrm{~m}, 2 \mathrm{H}), 1.35-1.30(\mathrm{~m}, 4 \mathrm{H}), 0.91-0.84$ (m, 3H) ppm. ${ }^{13} \mathbf{C ~ N M R}\left(101 \mathrm{MHz}, \mathrm{CDCl}_{3}, 298 \mathrm{~K}\right) \delta_{\mathrm{C}} 200.9,53.9,50.6,44.3,31.6,28.7,26.5$, 24.4, 22.5, $14.0 \mathrm{ppm}$. HRMS $m / z$ (DART): calculated for $\mathrm{C}_{10} \mathrm{H}_{20} \mathrm{NS}(\mathrm{M}+\mathrm{H})$ : 186.1311 ; found: 186.1298; IR (neat): 2953, 2926, 2875, 1646, 1469, 1445, 1328, 1257, 1218, 1174, 1085, 969, $895,824,728 \mathrm{~cm}^{-1} ; \mathbf{R}_{\mathbf{f}}\left(9: 1\right.$ hexanes/EtOAc; $\left.\mathrm{KMnO}_{4}\right): 0.36$. 


\section{F. Synthesis of organozinc reagents}

\section{General procedure D: Preparation of organozinc reagents}

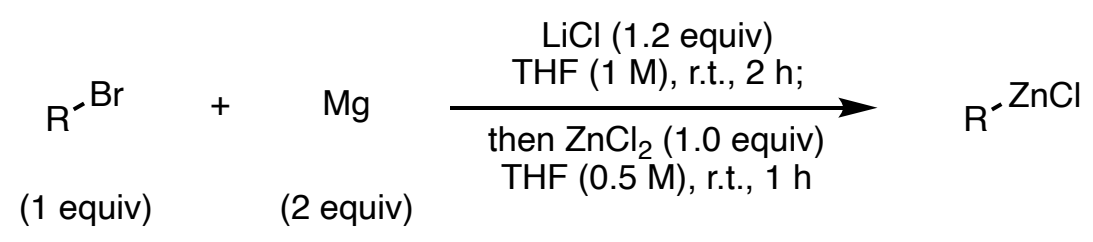

Preparation of the Grignard reagent: To a flame-dried $25-\mathrm{mL}$ round bottom flask with a stir bar was added magnesium( 0$)$ turnings $(0.49 \mathrm{~g}, 20 \mathrm{mmol}, 2.0$ equiv. $)$ and lithium chloride $(0.51 \mathrm{~g}, 12$ mmol, 1.2 equiv.). The flask was sealed with a size-14 septum and electrical tape and was flamedried under vacuum and cooled under $\mathrm{N}_{2}$. THF $(10 \mathrm{~mL}, 1.0 \mathrm{M})$ was added. The tube was equipped with syringes containing 1,2-dibromoethane (ca. $0.020 \mathrm{~mL}, 0.23 \mathrm{mmol}, 0.023$ equiv.) and the desired aryl bromide (10 mmol, 1.0 equiv). (If the aryl bromide was solid, the substrate was prepared as a stock solution in $3 \mathrm{~mL}$ of THF and $7 \mathrm{~mL}$ of THF as reaction solvent was used instead of $10 \mathrm{~mL}$ ) The magnesium was activated with 1,2-dibromoethane and the aryl bromide was added portionwise over $1 \mathrm{~h}$ at r.t. The reaction was stirred for an additional $1 \mathrm{~h}$ at r.t. to yield the Grignard reagent as a grey-black solution.

Preparation of the arylzinc reagent: A flame-dried 25-mL round bottom flask with a stir bar was sealed with size-14 septum, evacuated and backfilled with $\mathrm{N}_{2}(\times 3)$, and was brought into a glovebox. Zinc(II) chloride (1.4 g, $10 \mathrm{mmol}, 1.0$ equiv.) was added and the tube was again sealed with the size-14 septum and electrical tape and was removed from the glovebox. THF $(10 \mathrm{~mL}$, $1.0 \mathrm{M}$ ) was added and the mixture was stirred at r.t. for $1 \mathrm{~h}$ to yield a $1.0 \mathrm{M}$ solution of $\mathrm{ZnCl}_{2}$ in THF as a cloudy-white solution. While stirring, the solution of Grignard reagent was transferred to the solution of $\mathrm{ZnCl}_{2}$ using a syringe. After addition, the solution was stirred for $1 \mathrm{~h}$ at r.t. to yield the arylzinc reagent as a light-grey solution. The solution was allowed to stand for $1 \mathrm{~h}$ before use and was titrated using $\mathrm{I}_{2}(0.20-0.40 \mathrm{M}){ }^{1}$ 
Scale and concentration of organozinc reagents prepared for this study:<smiles>COc1ccc([Ge]Cl)cc1</smiles>

$2 a$

10-mmol scale

[0.25-0.45 M]<smiles>Fc1ccc([Ge]Cl)cc1</smiles>

$2 e$

5-mmol scale [0.30 M]<smiles>Cl[GeH2]c1ccc(N(Cc2ccccc2)c2ccccc2)cc1</smiles>

$2 b$

6-mmol scale

[0.24 M]<smiles>COc1ccc([Ge]Cl)cc1OC</smiles>

$2 f$

6-mmol scale

[0.34 M]<smiles>Cl[Ge]c1ccc2ccccc2c1</smiles>

2c

6-mmol scale

[0.37 M]<smiles>Cc1ccc2ccc([Ge]Cl)cc2c1</smiles>

$2 \mathrm{~g}$

5-mmol scale [0.28 M]<smiles>Cl[Ge]c1ccsc1</smiles>

2d

6-mmol scale

[0.34 M]

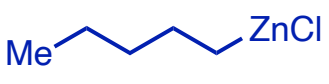

$2 \mathrm{~h}$

10-mmol scale

[0.35 M] 


\section{G. Synthesis of $O$-alkyl xanthate ester starting materials}

\section{General procedure E: Preparation of $\boldsymbol{O}$-alkyl xanthate starting materials}

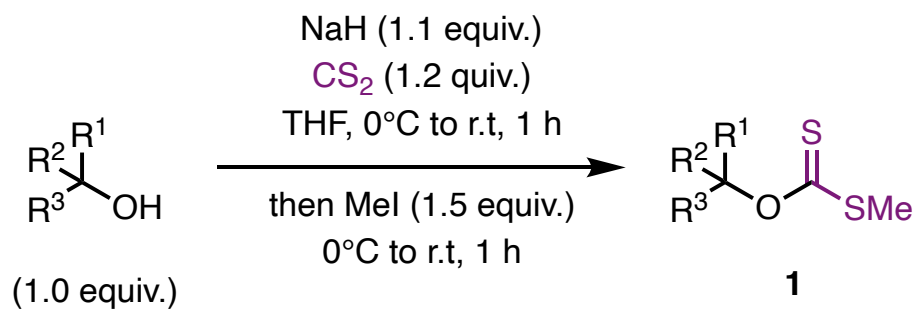

To a flame-dried $25-\mathrm{mL}$ flask with a stir bar was added alcohol substrate (1.0 equiv). The flask was sealed with a septum, and evacuated and backfilled with $\mathrm{N}_{2}(\times 3)$. THF $(0.33 \mathrm{M})$ was added and the solution was cooled to $0{ }^{\circ} \mathrm{C}$ while stirring. $\mathrm{CS}_{2}(1.2$ equiv) was added, followed by sodium hydride $(60 \% \mathrm{w} / \mathrm{w}$ dispersion in mineral oil, 1.1 equiv), and the reaction was allowed to warm to r.t. while stirring over $1 \mathrm{~h}$. The reaction was cooled to $0{ }^{\circ} \mathrm{C}$ and iodomethane (1.5 equiv) was added. The reaction was allowed to warm to r.t. while stirring over $1 \mathrm{~h}$. The reaction was quenched with sat. aq. $\mathrm{NH}_{4} \mathrm{Cl}$ and extracted with EtOAc $(\times 3)$. The organic fractions were combined, washed with brine $(\times 1)$, dried over $\mathrm{MgSO}_{4}$, and concentrated (The sulfur-containing aqueous waste was treated with bleach and disposed of appropriately.) The combined organic phase was concentrated, and the crude residue was purified by flash column chromatography to yield the desired xanthate ester product.

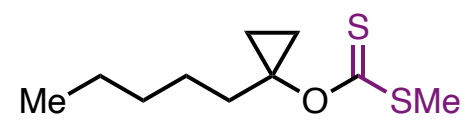

\section{$1 \mathrm{a}$}

$S$-methyl $O$-(1-pentylcyclopropyl) carbonodithioate (1a). Prepared on 10-mmol scale following General Procedure E. The crude residue was purified by flash column chromatography (gradient of 0-5\% EtOAc/hexanes) to yield the product as a yellow oil $(1.4 \mathrm{~g}, 6.5 \mathrm{mmol}, 65 \%)$. ${ }^{1} \mathbf{H}$ NMR $\left(400 \mathrm{MHz}, \mathrm{CDCl}_{3}, 298 \mathrm{~K}\right): \delta_{\mathrm{H}} 2.52(\mathrm{~s}, 3 \mathrm{H}), 2.02-1.92(\mathrm{~m}, 2 \mathrm{H}), 1.49-1.37(\mathrm{~m}, 2 \mathrm{H})$, $1.33-1.26(\mathrm{~m}, 4 \mathrm{H}), 1.16-1.01(\mathrm{~m}, 2 \mathrm{H}), 0.94-0.80(\mathrm{~m}, 3 \mathrm{H}), 0.80-0.73(\mathrm{~m}, 2 \mathrm{H}) \mathrm{ppm} .{ }^{13} \mathbf{C}$ NMR $\left(101 \mathrm{MHz}, \mathrm{CDCl}_{3}, 298 \mathrm{~K}\right): \delta_{\mathrm{C}} 214.5,68.3,33.6,31.7,25.7,22.8,19.3,14.2,12.9,12.9 \mathrm{ppm}$; HRMS $m / z$ (DART): calculated for $\mathrm{C}_{10} \mathrm{H}_{19} \mathrm{OS}_{2}(\mathrm{M}+\mathrm{H}): 219.0872$; found: 219.0870 ; IR (neat): 3092, 3011, 2958, 2930, 2858, 1433, 1419, 1381, 1315, 1196, 1171, 1053, 1020, 959, $726 \mathrm{~cm}^{-1}$; $\mathbf{R}_{\mathbf{f}}$ (95:5 hexanes/EtOAc; Vanillin): 0.90 .

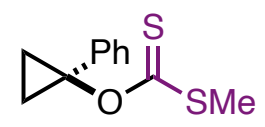

\section{$1 \mathrm{~b}$}

$S$-methyl $O$-(1-phenylcyclopropyl) carbonodithioate (1b). Prepared on 10-mmol scale following General Procedure E. The crude residue was purified by flash column chromatography 
(gradient of 0-5\% EtOAc/hexanes) to yield the product as a yellow oil (0.98 g, $4.4 \mathrm{mmol}, 44 \%)$. ${ }^{1} \mathbf{H}$ NMR $\left(400 \mathrm{MHz}, \mathrm{CDCl}_{3}, 298 \mathrm{~K}\right): \delta_{\mathrm{H}} 7.35-7.20(\mathrm{~m}, 5 \mathrm{H}), 2.53(\mathrm{~s}, 3 \mathrm{H}), 1.59-1.52(\mathrm{~m}, 2 \mathrm{H})$, 1.41-1.34 (m, 2H) ppm. ${ }^{13} \mathbf{C}$ NMR (100 MHz, $\left.\mathrm{CDCl}_{3}, 298 \mathrm{~K}\right): \delta_{\mathrm{C}} 214.2,139.5,128.4,127.1$, 125.6, 67.4, 19.6, $16.9 \mathrm{ppm}$. HRMS $m / z$ (DART): calculated for $\mathrm{C}_{11} \mathrm{H}_{13} \mathrm{OS}_{2}(\mathrm{M}+\mathrm{H}): 225.0402$; found: 225.0399; IR (neat): 3095, 3063, 3031, 3011, 2923, 1739, 1604, 1497, 1452, 1225, 1183 , $1057,959,895,752,695,566 \mathrm{~cm}^{-1} ; \mathbf{R}_{\mathbf{f}}\left(9: 1\right.$ hexanes/EtOAc; $\left.\mathrm{KMnO}_{4}\right): 0.67$.<smiles>CC(Cc1ccccc1)OC(=S)N(C)C</smiles>

$1 \mathrm{c}$

$S$-methyl $O$-(1-phenylpropan-2-yl) carbonodithioate (1c). Prepared on 3.0-mmol scale following General Procedure E. The crude residue was purified by flash column chromatography (gradient of $0-10 \%$ EtOAc/hexanes) to yield the product as a yellow oil $(0.59 \mathrm{~g}, 2.6 \mathrm{mmol}$, 87\%). ${ }^{1} \mathbf{H}$ NMR $\left(500 \mathrm{MHz}, \mathrm{CDCl}_{3}, 298 \mathrm{~K}\right): \delta_{\mathrm{H}} 7.33-7.28(\mathrm{~m}, 2 \mathrm{H}), 7.26-7.22(\mathrm{~m}, 3 \mathrm{H}), 5.95-5.85$ $(\mathrm{m}, 1 \mathrm{H}), 3.18(\mathrm{dd}, J=13.7,5.9 \mathrm{~Hz}, 1 \mathrm{H}), 2.89$ (dd, $J=13.7,7.0 \mathrm{~Hz}, 1 \mathrm{H}), 2.53(\mathrm{~s}, 3 \mathrm{H}), 1.36(\mathrm{~d}, J$ $=6.3 \mathrm{~Hz}, 3 \mathrm{H}) \mathrm{ppm} .{ }^{13} \mathbf{C} \mathbf{N M R}\left(126 \mathrm{MHz}, \mathrm{CDCl}_{3}, 298 \mathrm{~K}\right): \delta_{\mathrm{C}} 215.3,137.0,129.6,128.6,126.8$, 81.4, 41.7, 18.9, $18.8 \mathrm{ppm}$. HRMS $\mathrm{m} / z$ (DART): calculated for $\mathrm{C}_{11} \mathrm{H}_{15} \mathrm{OS}_{2}(\mathrm{M}+\mathrm{H}): 227.0559$; found: 227.0556; IR (neat): 3070, 3027, 2978, 2924, 1739, 1607, 1495, 1378, 1227, 1205, 1047, 963, 914, 744, 699, 620, $563 \mathrm{~cm}^{-1}$; $\mathbf{R}_{\mathbf{f}}$ (9:1 hexanes/EtOAc; Vanillin): 0.72.<smiles>CC(=S)OC1CCCCC1</smiles>

1d

$\boldsymbol{O}$-cyclohexyl $\boldsymbol{S}$-methyl carbonodithioate (1d). Prepared on 5.0-mmol scale following General Procedure E. The crude residue was purified by flash column chromatography (gradient of 0-5\% EtOAc/hexanes) to yield the product as a yellow oil (0.52 g, $2.7 \mathrm{mmol}, 54 \%) .{ }^{1} \mathbf{H}$ NMR (400 $\left.\mathrm{MHz}, \mathrm{CDCl}_{3}, 298 \mathrm{~K}\right): \delta_{\mathrm{H}} 5.57(\mathrm{tt}, J=8.8,3.9 \mathrm{~Hz}, 1 \mathrm{H}), 2.54(\mathrm{~s}, 3 \mathrm{H}), 2.04-1.93(\mathrm{~m}, 2 \mathrm{H}), 1.82-$ $1.69(\mathrm{~m}, 2 \mathrm{H}), 1.68-1.50(\mathrm{~m}, 3 \mathrm{H}), 1.49-1.24$ (m, 3H) ppm. ${ }^{13} \mathbf{C} \mathbf{N M R}\left(100 \mathrm{MHz}, \mathrm{CDCl}_{3}, 298 \mathrm{~K}\right)$ : $\delta_{\mathrm{C}} 215.2,82.6,31.1,25.4,23.7,18.9 \mathrm{ppm}$. HRMS $\mathrm{m} / z$ (DART): calculated for $\mathrm{C}_{8} \mathrm{H}_{15} \mathrm{OS}_{2}$ $(\mathrm{M}+\mathrm{H}):$ 191.0570; found: 191.0568; IR (neat): 2930, 2857, 1737, 1449, 1360, 1317, 1215, 1149 , 1113, 1050, 1021, 1002, 963, 917, 728, $562 \mathrm{~cm}^{-1}$; $\mathbf{R}_{\mathbf{f}}$ (9:1 hexanes/EtOAc; Vanillin): 0.80<smiles>CC(=S)OC(C)CC(=O)Nc1ccccc1</smiles>

$S$-methyl $O$-(4-oxo-4-(phenylamino)butan-2-yl) carbonodithioate (1e). Prepared on 3.0-mmol scale following General Procedure E. The crude residue was purified by flash column chromatography (gradient of 0-20\% EtOAc/hexanes) to yield the product as an off-white solid (0.51 g, 1.9 mmol, 63\%). ${ }^{1} \mathbf{H}$ NMR (500 MHz, $\left.\mathrm{CDCl}_{3}, 298 \mathrm{~K}\right): \delta_{\mathrm{H}} 7.54$ (s (br), $\left.1 \mathrm{H}\right), 7.52-7.48$ 
(m, 2H), 7.34-7.29 (m, 2H), 7.15-7.08 (m, 1H), $6.07(\mathrm{~h}, J=6.3 \mathrm{~Hz}, 1 \mathrm{H}), 2.90(\mathrm{dd}, J=14.6,6.3$ $\mathrm{Hz}, 1 \mathrm{H}), 2.70(\mathrm{dd}, J=14.6,5.9 \mathrm{~Hz}, 1 \mathrm{H}), 2.56(\mathrm{~s}, 3 \mathrm{H}), 1.52(\mathrm{~d}, J=6.3 \mathrm{~Hz}, 3 \mathrm{H}) \mathrm{ppm} .{ }^{13} \mathbf{C} \mathbf{N M R}$ $\left(126 \mathrm{MHz} \mathrm{CDCl}_{3}, 298 \mathrm{~K}\right): \delta_{\mathrm{C}} 215.3,167.3,137.7,129.1,124.7,120.1,77.7,43.9,19.5,19.2$ ppm. HRMS $m / z$ (DART): calculated for $\mathrm{C}_{12} \mathrm{H}_{16} \mathrm{NO}_{2} \mathrm{~S}_{2}(\mathrm{M}+\mathrm{H}): 270.0617$; found: 270.0623 ; IR (neat): 3245, 3193, 3134, 3046, 2977, 2916, 1654, 1597, 1498, 1378, 1316, 1234, 1209, 1054, $1026,965,738,693,556 \mathrm{~cm}^{-1} ; \mathbf{R}_{\mathbf{f}}\left(8: 2\right.$ hexanes/EtOAc; $\left.\mathrm{KMnO}_{4}\right): 0.44$.<smiles>CSC(=S)OCCCc1ccccc1</smiles>

$1 f$

$\boldsymbol{S}$-methyl $\boldsymbol{O}$-(3-phenylpropyl) carbonodithioate (1f). Prepared on 5.0-mmol scale following General Procedure E. The crude residue was purified by flash column chromatography (gradient of $0-5 \%$ EtOAc/hexanes) to yield the product as a pale-yellow oil $(1.0 \mathrm{~g}, 4.6 \mathrm{mmol}, 92 \%) .{ }^{1} \mathbf{H}$ NMR $\left(400 \mathrm{MHz}, \mathrm{CDCl}_{3}, 298 \mathrm{~K}\right): \delta_{\mathrm{H}} 7.34-7.27(\mathrm{~m}, 2 \mathrm{H}), 7.23-7.19(\mathrm{~m}, 3 \mathrm{H}), 4.62(\mathrm{t}, J=6.4 \mathrm{~Hz}$, 2H), $2.76(\mathrm{dd}, J=8.5,6.7 \mathrm{~Hz}, 2 \mathrm{H}), 2.57(\mathrm{~s}, 3 \mathrm{H}), 2.17-2.10(\mathrm{~m}, 2 \mathrm{H}) \mathrm{ppm} ;{ }^{13} \mathbf{C}$ NMR $(101 \mathrm{MHz}$, $\left.\mathrm{CDCl}_{3}, 298 \mathrm{~K}\right): \delta_{\mathrm{C}} 216.1,141.0,128.7,128.6,126.3,73.3,32.3,30.0,19.1 \mathrm{ppm} ; \mathbf{H R M S ~} \mathrm{m} / \mathrm{z}$ (DART): calculated for $\mathrm{C}_{11} \mathrm{H}_{15} \mathrm{OS}_{2}(\mathrm{M}+\mathrm{H}): 227.0559$; found: 227.0555; IR (neat): 3067, 3026, 2958, 2931, 2857, 1602, 1495, 1453, 1214, 1055, 964, 744, $698 \mathrm{~cm}^{-1}$; $\mathbf{R}_{\mathbf{f}}(95: 5$ hexanes/EtOAc; $\left.\mathrm{KMnO}_{4}\right): 0.45$.<smiles>CSC(=S)OCCc1ccccn1</smiles>

$1 \mathrm{~g}$

$\boldsymbol{S}$-methyl $\boldsymbol{O}$-(2-(pyridin-2-yl)ethyl) carbonodithioate (1g). Prepared on 3.0-mmol scale following General Procedure E. The crude residue was purified by flash column chromatography (gradient of 0-30\% EtOAc/hexanes) to yield the product as a yellow oil $(0.63 \mathrm{~g}, 3.0 \mathrm{mmol}$, 99\%). ${ }^{1} \mathbf{H}$ NMR $\left(500 \mathrm{MHz}, \mathrm{CDCl}_{3}, 298 \mathrm{~K}\right): \delta_{\mathrm{H}} 8.55(\mathrm{ddt}, J=4.9,1.7,0.9 \mathrm{~Hz}, 1 \mathrm{H}), 7.62(\mathrm{tdd}, J=$ $7.7,1.9,0.9 \mathrm{~Hz}, 1 \mathrm{H}), 7.21(\mathrm{dt}, J=7.8,1.0 \mathrm{~Hz}, 1 \mathrm{H}), 7.16$ (ddt, $J=7.4,4.9,1.1 \mathrm{~Hz}, 1 \mathrm{H}), 5.01-$ $4.95(\mathrm{~m}, 2 \mathrm{H}), 3.28(\mathrm{t}, J=6.7 \mathrm{~Hz}, 2 \mathrm{H}), 2.49$ (s, 3H) ppm. ${ }^{13} \mathbf{C} \mathbf{N M R}\left(126 \mathrm{MHz}, \mathrm{CDCl}_{3}, 298 \mathrm{~K}\right)$ : $\delta_{\mathrm{C}} 215.7,157.6,149.7,136.6,123.6,121.9,72.8,37.0,19.0 \mathrm{ppm}$. HRMS $\mathrm{m} / z$ (DART): calculated for $\mathrm{C}_{9} \mathrm{H}_{12} \mathrm{NOS}_{2}(\mathrm{M}+\mathrm{H}): 214.0355$; found: 214.0356; IR (neat): 3074, 3007, 2960, 2920, 1738, 1569, 1590, 1474, 1205, 1057, 965, 753, 626, $568 \mathrm{~cm}^{-1}$; $\mathbf{R}_{\mathbf{f}}(95: 5$ hexanes/EtOAc; $\left.\mathrm{KMnO}_{4}\right): 0.38$.<smiles>C=CCCOC(=S)OC</smiles>

$1 \mathrm{~h}$

$\boldsymbol{O}$-(but-3-en-1-yl) $\boldsymbol{S}$-methyl carbonodithioate (1h). Prepared on 3.0-mmol scale following General Procedure E. The crude residue was purified by flash column chromatography (gradient of $0-5 \%$ EtOAc/hexanes $)$ to yield the product as a yellow oil $(0.42 \mathrm{~g}, 2.6 \mathrm{mmol}, 86 \%)$. ${ }^{1} \mathbf{H}$ NMR $\left(400 \mathrm{MHz}, \mathrm{CDCl}_{3}, 298 \mathrm{~K}\right): \delta_{\mathrm{H}} 5.83(\mathrm{ddt}, J=17.0,10.2,6.7 \mathrm{~Hz}, 1 \mathrm{H}), 5.21-5.08(\mathrm{~m}, 2 \mathrm{H}), 4.64(\mathrm{t}$, $J=6.7 \mathrm{~Hz}, 2 \mathrm{H}), 2.60-2.52(\mathrm{~m}, 5 \mathrm{H}) \mathrm{ppm} .{ }^{13} \mathrm{C} \mathbf{N M R}\left(101 \mathrm{MHz}, \mathrm{CDCl}_{3}, 298 \mathrm{~K}\right): \delta_{\mathrm{C}} 216.0,133.5$, 
117.8, 72.9, 32.7, 19.0 ppm. HRMS $m / z$ (DART): calculated for $\mathrm{C}_{6} \mathrm{H}_{11} \mathrm{OS}_{2}(\mathrm{M}+\mathrm{H}): 163.0246$; found: 163.0247; IR (neat): 3085, 2976, 2920, 1739, 1642, 1421, 1372, 1205, 1140, 1057, 993, 965, 917, $631 \mathrm{~cm}^{-1} ; \mathbf{R}_{\mathbf{f}}\left(95: 5\right.$ hexanes/EtOAc; $\left.\mathrm{KMnO}_{4}\right): 0.66$.<smiles>C#CCCOC(=S)O[Na]</smiles>

$1 \mathrm{i}$

$\boldsymbol{O}$-(but-3-yn-1-yl) $\boldsymbol{S}$-methyl carbonodithioate (1i). Prepared on 3.0-mmol scale following General Procedure E. The crude residue was purified by flash column chromatography (gradient of $0-5 \%$ EtOAc/hexanes) to yield the product as a yellow oil $(0.48 \mathrm{~g}, 3.0 \mathrm{mmol}, 99 \%) .{ }^{1} \mathbf{H}$ NMR $\left(500 \mathrm{MHz}, \mathrm{CDCl}_{3}, 298 \mathrm{~K}\right): \delta_{\mathrm{H}} 4.69(\mathrm{t}, J=6.8 \mathrm{~Hz}, 2 \mathrm{H}), 2.71(\mathrm{td}, J=6.9,2.7 \mathrm{~Hz}, 2 \mathrm{H}), 2.57(\mathrm{~s}$, $3 \mathrm{H}), 2.04(\mathrm{t}, J=2.7 \mathrm{~Hz}, 1 \mathrm{H}) \mathrm{ppm} .{ }^{13} \mathbf{C}$ NMR $\left(126 \mathrm{MHz}, \mathrm{CDCl}_{3}, 298 \mathrm{~K}\right) \delta_{\mathrm{C}} 215.8,79.5,70.8$, 70.5, 19.2, 18.7. HRMS $m / z$ (DART): calculated for $\mathrm{C}_{6} \mathrm{H}_{9} \mathrm{OS}_{2}(\mathrm{M}+\mathrm{H})$ : 161.0089 ; found: 161.0087; IR (neat): 3292, 2958, 2920, 1738, 1419, 1339, 1321, 1205, 1175, 1074, 1047, 968, $644 \mathrm{~cm}^{-1} ; \mathbf{R}_{\mathbf{f}}\left(9: 1\right.$ hexanes/EtOAc; $\left.\mathrm{KMnO}_{4}\right): 0.20$.<smiles>CC(=S)OC(C)c1ccc(Br)cc1</smiles>

$1 \mathrm{j}$

$\boldsymbol{O}$-(1-(4-bromophenyl)ethyl) $S$-methyl carbonodithioate (1j). Prepared on 4.4-mmol scale following General Procedure E. The crude residue was purified by flash column chromatography (gradient of $0-5 \%$ EtOAc/hexanes) to yield the product as a yellow oil $(0.80 \mathrm{~g}, 2.7 \mathrm{mmol}, 62 \%)$. ${ }^{1} \mathbf{H}$ NMR $\left(500 \mathrm{MHz}, \mathrm{CDCl}_{3}, 298 \mathrm{~K}\right): \delta_{\mathrm{H}} 7.51-7.47(\mathrm{~m}, 2 \mathrm{H}), 7.27-7.24(\mathrm{~m}, 2 \mathrm{H}), 6.60$ (q, $J=6.6$ $\mathrm{Hz}, 1 \mathrm{H}), 2.56(\mathrm{~s}, 3 \mathrm{H}), 1.67(\mathrm{~d}, J=6.6 \mathrm{~Hz}, 3 \mathrm{H}) \mathrm{ppm} .{ }^{13} \mathbf{C} \mathbf{~ N M R}\left(126 \mathrm{MHz}, \mathrm{CDCl}_{3}, 298 \mathrm{~K}\right): \delta_{\mathrm{C}}$ 215.1, 139.8, 131.9, 128.3, 122.2, 80.7, 22.0, $19.2 \mathrm{ppm}$. HRMS $\mathrm{m} / \mathrm{z}$ (DART): calculated for $\mathrm{C}_{10} \mathrm{H}_{15} \mathrm{BrNOS}_{2}\left(\mathrm{M}+\mathrm{NH}_{4}\right)$ : 307.9773; found: 307.9783 ; IR (neat): 2980, 2923, 1734, 1642, 1594, 1488, 1406, 1373, 1209, 1119, 1094, 1009, 822, 779, $562 \mathrm{~cm}^{-1} ; \mathbf{R}_{\mathbf{f}}$ (95:5 hexanes/EtOAc; $\left.\mathrm{KMnO}_{4}\right): 0.85$.<smiles>CC(=S)OC(C)c1ccccc1</smiles>

$1 \mathrm{k}$

$S$-methyl $O$-(1-phenylethyl) carbonodithioate (1k). Prepared on 5.0-mmol scale following General Procedure E. The crude residue was purified by flash column chromatography (gradient of $0-5 \%$ EtOAc/hexanes) to yield the product as a yellow oil $(1.01 \mathrm{~g}, 3.7 \mathrm{mmol}, 74 \%) .{ }^{1} \mathbf{H}$ NMR (400 MHz, $\left.\mathrm{CDCl}_{3}, 298 \mathrm{~K}\right): \delta_{\mathrm{H}} 7.41-7.34(\mathrm{~m}, 4 \mathrm{H}), 7.34-7.28(\mathrm{~m}, 1 \mathrm{H}), 6.66(\mathrm{q}, J=6.6 \mathrm{~Hz}, 1 \mathrm{H})$, $2.55(\mathrm{~s}, 3 \mathrm{H}), 1.69(\mathrm{~d}, J=6.6 \mathrm{~Hz}, 3 \mathrm{H}) \mathrm{ppm} .{ }^{13} \mathbf{C} \mathbf{N M R}\left(100 \mathrm{MHz}, \mathrm{CDCl}_{3}, 298 \mathrm{~K}\right): \delta_{\mathrm{C}} 215.1$, 140.7, 128.7, 128.3, 126.6, 81.6, 22.0, $19.1 \mathrm{ppm}$. HRMS $\mathrm{m} / z$ (DART): calculated for $\mathrm{C}_{10} \mathrm{H}_{13} \mathrm{OS}_{2}$ $(\mathrm{M}+\mathrm{H}): 213.0402$; found: 213.0406; IR (neat): 3039, 2980, 2923, 1494, 1451, 1419, 1374, 1217, 1200, 968, 963, 759, 697, $559 \mathrm{~cm}^{-1} ; \mathbf{R}_{\mathbf{f}}(9: 1$ hexanes/EtOAc; Vanillin): 0.85 . 
<smiles>CC(=S)OC(Cl)c1ccccc1</smiles>

11

$\boldsymbol{O}$-(cyclohexyl(phenyl)methyl) $\boldsymbol{S}$-methyl carbonodithioate (11). Prepared on 5.0-mmol scale following General Procedure E. The crude residue was purified by flash column chromatography (gradient of $0-2.5 \%$ EtOAc/hexanes) to yield the product as a yellow oil $(0.14 \mathrm{~g}, 0.48 \mathrm{mmol}$, $10 \%) .{ }^{1} \mathbf{H}$ NMR $\left(500 \mathrm{MHz}, \mathrm{CDCl}_{3}, 298 \mathrm{~K}\right): \delta_{\mathrm{H}} 7.35-7.26(\mathrm{~m}, 5 \mathrm{H}), 6.29(\mathrm{~d}, J=7.8 \mathrm{~Hz}, 1 \mathrm{H}), 2.55$ $(\mathrm{s}, 3 \mathrm{H}), 2.03-1.86(\mathrm{~m}, 2 \mathrm{H}), 1.79-1.74(\mathrm{~m}, 1 \mathrm{H}), 1.71-1.63(\mathrm{~m}, 2 \mathrm{H}), 1.46-1.38(\mathrm{~m}, 1 \mathrm{H}), 1.26-1.10$ (m, 4H), 1.03-0.93 (m, 1H) ppm. ${ }^{13} \mathbf{C}$ NMR (126 MHz, $\left.\mathrm{CDCl}_{3}, 298 \mathrm{~K}\right): \delta_{\mathrm{C}} 215.4,138.4,128.4$, 128.1 127.7, 89.6, 43.4, 29.2, 29.1, 26.4, 26.0, 25.9, 19.1 ppm. EA: calculated for $\mathrm{C}_{15} \mathrm{H}_{20} \mathrm{OS}_{2}$ : \%C: $64.2, \% \mathrm{H}: 7.2 \%, \% \mathrm{~N}: 0.0$; found: $\% \mathrm{C}: 64.6, \% \mathrm{H}: 7.7 \%, \% \mathrm{~N}: 0.0$; IR (neat): 3061, 3032, 2921, 2848, 1709, 1450, 1357, 1333, 1216,1150, 1038, 968, 885, 693, $581 \mathrm{~cm}^{-1} ; \mathbf{R}_{\mathbf{f}}(95: 5$ hexanes/EtOAc; $\mathrm{KMnO}_{4}$ ): 0.77.<smiles>CC(=S)OCc1ccccc1</smiles>

$1 \mathrm{~m}$

$\boldsymbol{O}$-benzyl $\boldsymbol{S}$-methyl carbonodithioate (1m). Prepared on 4.2-mmol scale following General Procedure E. The crude residue was purified by flash column chromatography (gradient of 0-2\% EtOAc/hexanes) to yield the product as an off-white solid (0.67 g, $3.4 \mathrm{mmol}, 81 \%) .{ }^{\mathbf{1}} \mathbf{H} \mathbf{~ N M R}$ $\left(500 \mathrm{MHz}, \mathrm{CDCl}_{3}, 298 \mathrm{~K}\right): \delta_{\mathrm{H}} 7.43-7.36(\mathrm{~m}, 5 \mathrm{H}), 5.64(\mathrm{~s}, 2 \mathrm{H}), 2.58(\mathrm{~s}, 3 \mathrm{H}) \mathrm{ppm} .{ }^{13} \mathrm{C}$ NMR $(126$ $\left.\mathrm{MHz}, \mathrm{CDCl}_{3}, 298 \mathrm{~K}\right): \delta_{\mathrm{C}} 215.9,134.9,128.8,128.8,128.7,75.3,19.3 \mathrm{ppm}$. HRMS $\mathrm{m} / z$ (DART): calculated for $\mathrm{C}_{9} \mathrm{H}_{11} \mathrm{OS}_{2}(\mathrm{M}+\mathrm{H}): 199.0246$; found: 199.0247; IR (neat): 3095, 3067, 3031, 2927, 2853, 1958, 1785, 1498, 1453, 1412, 1318, 1196, 1029, 964, 895, 746, 694, 557 $\mathrm{cm}^{-1} ; \mathbf{R}_{\mathbf{f}}\left(95: 5\right.$ hexanes/EtOAc; $\left.\mathrm{KMnO}_{4}\right): 0.90$.<smiles>CC(=S)OCc1ccc(Cl)cc1</smiles>

1n

$\boldsymbol{O}$-(4-chlorobenzyl) $\boldsymbol{S}$-methyl carbonodithioate (1n). Prepared on 3.0-mmol scale following General Procedure E. The crude residue was purified by flash column chromatography (gradient of $0-5 \%$ EtOAc/hexanes) to yield the product as an off-white solid (0.69 g, $2.9 \mathrm{mmol}, 98 \%) .{ }^{1} \mathbf{H}$ NMR (500 MHz, $\left.\mathrm{CDCl}_{3}, 298 \mathrm{~K}\right): \delta_{\mathrm{H}} 7.40-7.30(\mathrm{~m}, 4 \mathrm{H}), 5.60(\mathrm{~s}, 2 \mathrm{H}), 2.58(\mathrm{~s}, 3 \mathrm{H}) \mathrm{ppm} .{ }^{13} \mathbf{C}$ NMR (126 MHz, $\left.\mathrm{CDCl}_{3}, 298 \mathrm{~K}\right): \delta_{\mathrm{C}} 215.8,134.7,133.4,130.0,129.0,74.2,19.3 \mathrm{ppm}$. HRMS $\mathrm{m} / z$ (DART): calculated for $\mathrm{C}_{9} \mathrm{H}_{10} \mathrm{ClOS}_{2}(\mathrm{M}+\mathrm{H}): 232.9856$; found: 232.9860 ; IR (neat): 2922, 1599, 1492, 1409, 1366, 1316, 1194, 1172, 1058, 1015, 965, 849, 804, 735, $629 \mathrm{~cm}^{-1} ; \mathbf{R}_{\mathbf{f}}(9: 1$ hexanes/EtOAc; $\mathrm{KMnO}_{4}$ ): 0.90 . 
<smiles>CCOC(=O)C(C)OC(=S)OC</smiles>

10

Ethyl 2-(((methylthio)carbonothioyl)oxy)propanoate (10). Prepared on 4.0-mmol scale following General Procedure E. The crude residue was purified by flash column chromatography (gradient of 0-5\% EtOAc/hexanes) to yield the product as an off-white solid (0.48 $\mathrm{g}, 2.3 \mathrm{mmol}$, $58 \%) .{ }^{1} \mathbf{H}$ NMR $\left(500 \mathrm{MHz}, \mathrm{CDCl}_{3}, 298 \mathrm{~K}\right): \delta_{\mathrm{H}} 5.73(\mathrm{q}, J=7.1 \mathrm{~Hz}, 1 \mathrm{H}), 4.21(\mathrm{qd}, J=7.1,1.2$ $\mathrm{Hz}, 2 \mathrm{H}), 2.59$ (s, 3H), $1.63(\mathrm{~d}, J=7.0 \mathrm{~Hz}, 3 \mathrm{H}), 1.27(\mathrm{t}, J=7.1 \mathrm{~Hz}, 3 \mathrm{H}) \mathrm{ppm} .{ }^{13} \mathbf{C}$ NMR (126 $\left.\mathrm{MHz}_{\mathrm{CDCl}}, 298 \mathrm{~K}\right): \delta_{\mathrm{C}} 215.7,169.9,76.6,61.7,19.4,17.2,14.2 \mathrm{ppm}$. HRMS $\mathrm{m} / z$ (DART): calculated for $\mathrm{C}_{7} \mathrm{H}_{13} \mathrm{O}_{3} \mathrm{~S}_{2}(\mathrm{M}+\mathrm{H}): 209.0301$; found: 209.0301; IR (neat): 2985, 2934, 1746, 1447, 1374, 1298, 1268, 1202, 1123, 1058, 966, 860, 744, $679 \mathrm{~cm}^{-1} ; \mathbf{R}_{\mathbf{f}}(8: 2$ hexanes/EtOAc; $\left.\mathrm{KMnO}_{4}\right): 0.67$. 


\section{$\underline{\text { H. Synthesis of thiocarbonyl imidazolide starting materials }}$}

\section{General procedure F: Synthesis of thiocarbonyl imidazolide starting materials}

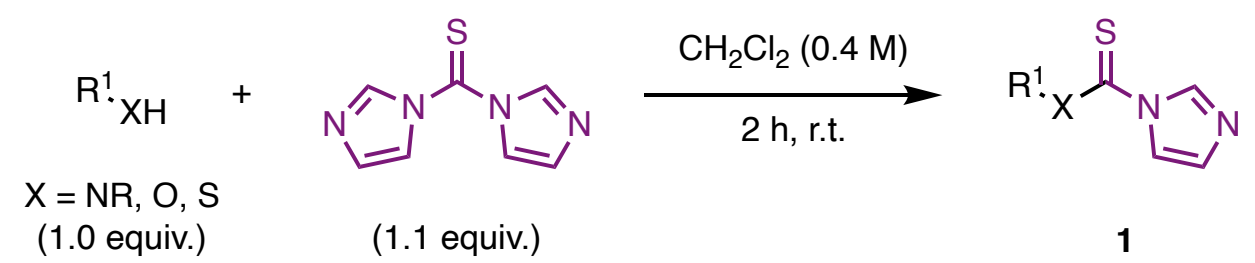

To a flame-dried $25-\mathrm{mL}$ flask with stir bar was added alcohol, amine or thiol starting material (1.0 equiv.). The flask was sealed, evacuated and backfilled with $\mathrm{N}_{2}(\times 3)$, and $\mathrm{CH}_{2} \mathrm{Cl}_{2}(0.40 \mathrm{M})$ was added. While the mixture was stirring, the flask was briefly opened to air and 1,1'thiocarbonyldiimidazole ( 1.1 equiv.) was added. The flask was sealed, wrapped in aluminum foil, and stirred at r.t. for $2 \mathrm{~h}$. The reaction was opened to air, quenched with sat. aq. $\mathrm{NH}_{4} \mathrm{Cl}$, and extracted with $\mathrm{CH}_{2} \mathrm{Cl}_{2}(\times 3)$. The organic fractions were combined, washed with brine $(\times 1)$, dried over $\mathrm{MgSO}_{4}$, and concentrated. (The sulfur-containing aqueous waste was treated with bleach and disposed of appropriately.) The crude residue was purified by flash column chromatography to yield desired product.<smiles>CCN(CC)C(=S)n1ccnc1</smiles>

$$
\text { 4a }
$$

$\mathrm{N}, \mathrm{N}$-Diethyl-1H-imidazole-1-carbothioamide (4a). Prepared on 3.0-mmol scale following General Procedure F. The crude residue was purified by flash column chromatography (gradient of 0-50\% EtOAc/hexanes) to yield the product as a white solid $(0.50 \mathrm{~g}, 2.9 \mathrm{mmol}, 95 \%)$.

${ }^{1} \mathbf{H}$ NMR $\left(400 \mathrm{MHz}, \mathrm{CDCl}_{3}, 298 \mathrm{~K}\right): \delta_{\mathrm{H}} 7.79(\mathrm{t}, J=1.1 \mathrm{~Hz}, 1 \mathrm{H}), 7.16(\mathrm{t}, J=1.4 \mathrm{~Hz}, 1 \mathrm{H}), 7.07$ $(\mathrm{dd}, J=1.5,0.9 \mathrm{~Hz}, 1 \mathrm{H}), 3.71$ (s (br), 4H), $1.31(\mathrm{t}, J=7.1 \mathrm{~Hz}, 6 \mathrm{H}) \mathrm{ppm} .{ }^{13} \mathrm{C}$ NMR $(101 \mathrm{MHz}$, $\mathrm{CDCl}_{3}, 298 \mathrm{~K}$ ): $\delta_{\mathrm{C}} 178.6,136.8,129.7,119.1,47.6,12.6 \mathrm{ppm}$. HRMS $\mathrm{m} / z$ (DART): calculated for $\mathrm{C}_{8} \mathrm{H}_{14} \mathrm{~N}_{3} \mathrm{~S}(\mathrm{M}+\mathrm{H}):$ 184.0903; found: 184.0903; IR (neat): 3119, 3100, 2997, 2930, 2871, $1643,1505,1472,1355,1273,1229,1147,1089,1050,913,821,770,655,574 \mathrm{~cm}^{-1} ; \mathbf{R}_{\mathbf{f}}(2: 8$ hexanes/EtOAc; $\mathrm{KMnO}_{4}$ ): 0.33 .<smiles>C=CCN(CC=C)C(=S)n1ccnc1</smiles>

$4 \mathrm{~b}$

$\mathrm{N}, \mathrm{N}$-Diallyl-1 $\mathrm{H}$-imidazole-1-carbothioamide (4b). Prepared on 3.0-mmol scale following General Procedure F. The crude residue was purified by flash column chromatography (gradient of $20-80 \%$ EtOAc/hexanes) to yield the product as a light-yellow solid ( $0.58 \mathrm{~g}, 2.8 \mathrm{mmol}, 93 \%)$. 
${ }^{1} \mathbf{H}$ NMR $\left(500 \mathrm{MHz}, \mathrm{CDCl}_{3}, 298 \mathrm{~K}\right): \delta_{\mathrm{H}} 7.90(\mathrm{~d}, J=1.3 \mathrm{~Hz}, 1 \mathrm{H}), 7.26(\mathrm{~d}, J=1.7 \mathrm{~Hz}, 1 \mathrm{H}), 7.06$ $(\mathrm{t}, J=1.2 \mathrm{~Hz}, 1 \mathrm{H}), 6.02-5.78(\mathrm{~m}, 2 \mathrm{H}), 5.38$ (dq, $J=10.3,1.3 \mathrm{~Hz}, 2 \mathrm{H}), 5.28(\mathrm{dq}, J=17.1,1.4$ $\mathrm{Hz}, 2 \mathrm{H}), 4.28$ (s (br), 4H) ppm. ${ }^{13} \mathbf{C}$ NMR (126 MHz, CDCl, $298 \mathrm{~K}$ ): $\delta_{\mathrm{C}} 180.0,137.3,130.6$, 129.8, 120.1, 119.4, 54.9 ppm. HRMS $m / z$ (DART): calculated for $\mathrm{C}_{10} \mathrm{H}_{14} \mathrm{~N}_{3} \mathrm{~S}(\mathrm{M}+\mathrm{H})$ : 208.0903; found: 208.0903; IR (neat): 3114, 3075, 2979, 1645, 1519, 1497, 1465, 1405, 1359, 1294, 1215, 1103, 1057, 986, 912, 819, 756, 648, $564 \mathrm{~cm}^{-1} ; \mathbf{R}_{\mathbf{f}}\left(3: 7\right.$ hexanes/EtOAc; $\left.\mathrm{KMnO}_{4}\right)$ : 0.57 .<smiles>S=C(N(Cc1ccccc1)Cc1ccccc1)n1ccnc1</smiles>

4c

$\mathrm{N}, \mathrm{N}$-Dibenzyl-1H-imidazole-1-carbothioamide (4c). Prepared on 2.0-mmol scale following General Procedure F. The crude residue was purified by flash column chromatography (gradient of $20-80 \%$ EtOAc/hexanes) to yield the product as a yellow oil $(0.42 \mathrm{~g}, 1.4 \mathrm{mmol}, 69 \%)$.

${ }^{1} \mathbf{H}$ NMR $\left(400 \mathrm{MHz}, \mathrm{CDCl}_{3}, 298 \mathrm{~K}\right): \delta_{\mathrm{H}} 8.00(\mathrm{~s}, 1 \mathrm{H}), 7.42-7.35(\mathrm{~m}, 7 \mathrm{H}), 7.26(\mathrm{~s}(\mathrm{br}), 1 \mathrm{H}), 7.20$ (s (br), 2H), 7.18 (s (br), 1H), 7.08 (s (br), 1H), 4.92 (s (br), 4H) ppm. ${ }^{13}$ C NMR (100 MHz, $\left.\mathrm{CDCl}_{3}, 298 \mathrm{~K}\right): \delta_{\mathrm{C}} 181.2,137.5,134.6,130.2,129.3,128.6,127.7,119.4,55.6 \mathrm{ppm}$. HRMS $\mathrm{m} / \mathrm{z}$ (DART): calculated for $\mathrm{C}_{18} \mathrm{H}_{18} \mathrm{~N}_{3} \mathrm{~S}(\mathrm{M}+\mathrm{H})$ : 308.1216; found: 308.1218; IR (neat): 3070, 3030, 2930, 1735, 1603, 1493, 1452, 1423, 1359, 1286, 1224, 1152, 1101, 1040, 946, 897, 820, 738, $697,596 \mathrm{~cm}^{-1} ; \mathbf{R}_{\mathbf{f}}\left(2: 8\right.$ hexanes/EtOAc; $\left.\mathrm{KMnO}_{4}\right): 0.82$.<smiles></smiles>

$4 d$

(1H-Imidazol-1-yl)(pyrrolidin-1-yl)methanethione (4d). Prepared on 5.0-mmol scale following General Procedure F. The crude residue was purified by flash column chromatography (gradient of 20-90\% EtOAc/hexanes) to yield the product as a white solid $(0.80 \mathrm{~g}, 4.4 \mathrm{mmol}$, $88 \%$ ). ${ }^{1} \mathrm{H}$ NMR $\left(500 \mathrm{MHz}, \mathrm{CDCl}_{3}, 298 \mathrm{~K}\right): \delta_{\mathrm{H}} 7.98(\mathrm{t}, J=1.1 \mathrm{~Hz}, 1 \mathrm{H}), 7.31$ (t, $\left.J=1.4 \mathrm{~Hz}, 1 \mathrm{H}\right)$, 7.08-7.04 (m, 1H), 3.91 (d, $J=7.4 \mathrm{~Hz}, 2 \mathrm{H}), 3.64$ (t, $J=6.7 \mathrm{~Hz}, 2 \mathrm{H}), 2.13-2.00$ (m, 4H) ppm. ${ }^{13}$ C NMR (126 MHz, $\left.\mathrm{CDCl}_{3}, 298 \mathrm{~K}\right): \delta_{\mathrm{C}} 175.7,137.0,129.5,119.0,54.9,53.6,26.7,24.6 \mathrm{ppm}$. HRMS $m / z$ (DART): calculated for $\mathrm{C}_{8} \mathrm{H}_{12} \mathrm{~N}_{3} \mathrm{~S}(\mathrm{M}+\mathrm{H}): 182.0746$; found: 182.0748 ; IR (neat): 3140, 3113, 2979, 2941, 2870, 1624, 1523, 1490, 1464, 1360, 1293, 1224, 1110, 1033, 975, 825, $742,653,608 \mathrm{~cm}^{-1} ; \mathbf{R}_{\mathbf{f}}\left(1: 9\right.$ hexanes/EtOAc; $\left.\mathrm{KMnO}_{4}\right): 0.34$.<smiles>S=C(N1CCOCC1)n1ccnc1</smiles>

(1H-Imidazol-1-yl)(morpholino)methanethione (4e). Prepared on 2.0-mmol scale following General Procedure F. The crude residue was purified by flash column chromatography (gradient 
of $0-50 \%$ EtOAc/hexanes) to yield the product as a white solid $(0.31 \mathrm{~g}, 1.6 \mathrm{mmol}, 80 \%) .{ }^{1} \mathbf{H}$ NMR $\left(400 \mathrm{MHz}, \mathrm{CDCl}_{3}, 298 \mathrm{~K}\right): \delta_{\mathrm{H}} 7.87(\mathrm{t}, J=1.1 \mathrm{~Hz}, 1 \mathrm{H}), 7.18(\mathrm{t}, J=1.4 \mathrm{~Hz}, 1 \mathrm{H}), 7.08(\mathrm{t}, J$ $=1.2 \mathrm{~Hz}, 1 \mathrm{H}), 3.95-3.88(\mathrm{~m}, 4 \mathrm{H}), 3.80-3.77(\mathrm{~m}, 4 \mathrm{H})$ ppm. ${ }^{13} \mathbf{C}$ NMR $\left(100 \mathrm{MHz}, \mathrm{CDCl}_{3}, 298\right.$ $\mathrm{K}): \delta_{\mathrm{C}} 179.0,137.4,130.2,119.3,66.4,52.2 \mathrm{ppm}$. HRMS $m / z$ (DART): calculated for $\mathrm{C}_{8} \mathrm{H}_{12} \mathrm{~N}_{3} \mathrm{OS}(\mathrm{M}+\mathrm{H}):$ 198.0696; found: 198.0702; IR (neat): 3124, 3111, 2968, 2844, 1735, 1624, 1493, 1442, 1359, 1298, 1282, 1222, 1013, 1040, 1026, 955, 822, 756, 649, $608 \mathrm{~cm}^{-1} ; \mathbf{R}_{\mathbf{f}}(1: 9$ hexanes/EtOAc; $\mathrm{KMnO}_{4}$ ): 0.29 .<smiles>N#CC1CCN(C(=S)n2ccnc2)CC1</smiles>

1-(1H-Imidazole-1-carbonothioyl)piperidine-4-carbonitrile (4f). Prepared on 2.0-mmol scale following General Procedure F. The crude residue was purified by flash column chromatography (gradient of 20-90\% EtOAc/hexanes) to yield the product as a light-yellow solid $(0.34 \mathrm{~g}, 1.5$ mmol, 76\%). ${ }^{1} \mathbf{H}$ NMR $\left(400 \mathrm{MHz}, \mathrm{CDCl}_{3}, 298 \mathrm{~K}\right) \delta_{\mathrm{H}} 7.86(\mathrm{t}, J=1.1 \mathrm{~Hz}, 1 \mathrm{H}), 7.17(\mathrm{t}, J=1.4 \mathrm{~Hz}$, 1H), 7.11-7.06 (m, 1H), 4.02-3.92 (m, 4H), $3.03(\mathrm{tt}, J=6.5,4.5 \mathrm{~Hz}, 1 \mathrm{H}), 2.13-1.94(\mathrm{~m}, 4 \mathrm{H})$ ppm. ${ }^{13} \mathrm{C}$ NMR $\left(100 \mathrm{MHz}, \mathrm{CDCl}_{3}, 298 \mathrm{~K}\right) \delta_{\mathrm{C}} 179.5,137.4,130.3,120.0,119.3,49.5,28.5,26.1$ ppm. HRMS $m / z$ (DART): calculated for $\mathrm{C}_{10} \mathrm{H}_{13} \mathrm{~N}_{4} \mathrm{~S}(\mathrm{M}+\mathrm{H}): 221.0855$; found: 221.0846; IR (neat): 3135, 3123, 2965, 2867, 2242, 1647, 1516, 1494, 1448, 1359, 1290, 1214, 1101, 1084, $1038,919,825,770,650 \mathrm{~cm}^{-1} ; \mathbf{R}_{\mathbf{f}}\left(2: 8\right.$ hexanes/EtOAc; $\left.\mathrm{KMnO}_{4}\right): 0.22$.<smiles>S=C(N1CCC2(CC1)OCCO2)n1ccnc1</smiles>

$4 \mathrm{~g}$

(1H-Imidazol-1-yl)(1,4-dioxa-8-azaspiro[4.5]decan-8-yl)methanethione (4g). Prepared on 2.0-mmol scale following General Procedure F. The crude residue was purified by flash column chromatography (gradient of $20-90 \%$ EtOAc/hexanes) to yield the product as a white solid $(0.24$ g, $0.94 \mathrm{mmol}, 47 \%) .{ }^{1} \mathbf{H}$ NMR $\left(400 \mathrm{MHz}, \mathrm{CDCl}_{3}, 298 \mathrm{~K}\right): \delta_{\mathrm{H}} 7.85(\mathrm{t}, J=1.1 \mathrm{~Hz}, 1 \mathrm{H}), 7.18(\mathrm{t}, J$ $=1.4 \mathrm{~Hz}, 1 \mathrm{H}), 7.06(\mathrm{t}, J=1.2 \mathrm{~Hz}, 1 \mathrm{H}), 3.98(\mathrm{~s}(\mathrm{br}), 8 \mathrm{H}), 1.90-1.75(\mathrm{~m}, 4 \mathrm{H}) \mathrm{ppm} .{ }^{13} \mathbf{C} \mathbf{N M R}(101$ $\left.\mathrm{MHz}_{2} \mathrm{CDCl}_{3}, 298 \mathrm{~K}\right): \delta_{\mathrm{C}} 178.9,137.4,130.0,119.4,106.1,64.8,49.8,35.1 \mathrm{ppm} . \mathbf{H R M S ~ m} / z$ (DART): calculated for $\mathrm{C}_{11} \mathrm{H}_{16} \mathrm{~N}_{3} \mathrm{O}_{2} \mathrm{~S}(\mathrm{M}+\mathrm{H}): 254.0958$; found: 254.0956; IR (neat): 3115 , $2962,2875,1735,1501,1451,1360,1229,1096,1022,934,899,825,744,647,631 \mathrm{~cm}^{-1}$; $\mathbf{R}_{\mathbf{f}}$ (2:8 hexanes/EtOAc; $\left.\mathrm{KMnO}_{4}\right): 0.25$.<smiles>S=C(N1CCc2ccccc21)n1ccnc1</smiles> 
(1H-Imidazol-1-yl)(indolin-1-yl)methanethione (4h). Prepared on 2.0-mmol scale following General Procedure F. The crude residue was purified by flash column chromatography (gradient of 0-70\% EtOAc/hexanes) to yield the product as a white solid $(0.43 \mathrm{~g}, 1.9 \mathrm{mmol}, 94 \%) .{ }^{1} \mathbf{H}$ NMR (400 MHz, $\mathrm{CDCl}_{3}, 298 \mathrm{~K}$ ): $\delta_{\mathrm{H}} 7.99(\mathrm{t}, J=1.2 \mathrm{~Hz}, 1 \mathrm{H}), 7.29$ (ddt, $\left.J=6.9,1.5,0.7 \mathrm{~Hz}, 1 \mathrm{H}\right)$, $7.24(\mathrm{t}, J=1.5 \mathrm{~Hz}, 1 \mathrm{H}), 7.18(\mathrm{dd}, J=1.6,1.0 \mathrm{~Hz}, 1 \mathrm{H}), 7.13-6.99(\mathrm{~m}, 2 \mathrm{H}), 6.05$ (s (br), 1H), 4.47 $(\mathrm{dd}, J=8.2,7.4 \mathrm{~Hz}, 2 \mathrm{H}), 3.23(\mathrm{t}, J=7.8 \mathrm{~Hz}, 2 \mathrm{H}) \mathrm{ppm} .{ }^{13} \mathbf{C} \mathbf{N M R}\left(101 \mathrm{MHz}, \mathrm{CDCl}_{3}, 298 \mathrm{~K}\right): \delta_{\mathrm{C}}$ $172.4,141.3,136.1,134.7,129.2,127.9,126.3,126.1,119.1,115.6,57.6,27.4$ ppm. HRMS $\mathrm{m} / \mathrm{z}$ (DART): calculated for $\mathrm{C}_{12} \mathrm{H}_{12} \mathrm{~N}_{3} \mathrm{~S}(\mathrm{M}+\mathrm{H}): 230.0746$; found: 230.0750; IR (neat): 3070, 3030, 2930, 1735, 1603, 1473, 1452, 1423, 1359, 1286, 1224, 1152, 1079, 1040, 946, 820, 738, 697, $652,596 \mathrm{~cm}^{-1} ; \mathbf{R}_{\mathbf{f}}\left(3: 7\right.$ hexanes/EtOAc; $\left.\mathrm{KMnO}_{4}\right): 0.69$.<smiles>S=C(N1CCCc2ccccc21)n1ccnc1</smiles>

$4 \mathbf{i}$

(3,4-Dihydroquinolin-1(2H)-yl)(1H-imidazol-1-yl)methanethione (3i). Prepared on 2.0-mmol scale following General Procedure F. The crude residue was purified by flash column chromatography (gradient of $0-70 \%$ EtOAc/hexanes) to yield the product as a white solid (0.32 g, $1.3 \mathrm{mmol}, 66 \%$ ). ${ }^{1} \mathbf{H}$ NMR (400 MHz, $\left.\mathrm{CDCl}_{3}, 298 \mathrm{~K}\right): \delta_{\mathrm{H}} 7.79(\mathrm{t}, J=1.2 \mathrm{~Hz}, 1 \mathrm{H}), 7.27-7.20$ $(\mathrm{m}, 2 \mathrm{H}), 7.13(\mathrm{td}, J=7.5,1.2 \mathrm{~Hz}, 1 \mathrm{H}), 7.05-6.93(\mathrm{~m}, 2 \mathrm{H}), 6.90(\mathrm{dd}, J=1.6,0.9 \mathrm{~Hz}, 1 \mathrm{H}), 6.52$ $(\mathrm{ddd}, J=8.2,1.2,0.5 \mathrm{~Hz}, 1 \mathrm{H}), 4.27(\mathrm{t}, J=6.9 \mathrm{~Hz}, 2 \mathrm{H}), 2.83(\mathrm{t}, J=6.5 \mathrm{~Hz}, 2 \mathrm{H}), 2.22-2.10(\mathrm{~m}$, $2 \mathrm{H}) \mathrm{ppm} .{ }^{13} \mathbf{C} \mathbf{N M R}\left(101 \mathrm{MHz}, \mathrm{CDCl}_{3}, 298 \mathrm{~K}\right): \delta_{\mathrm{C}} 177.0,139.5,137.4,133.0,128.9,128.6$, 127.6, 127.0, 122.8, 119.9, 52.8, 26.9, $24.6 \mathrm{ppm}$. HRMS $\mathrm{m} / z$ (DART): calculated for $\mathrm{C}_{13} \mathrm{H}_{14} \mathrm{~N}_{3} \mathrm{~S}$ (M+H): 244.0903; found: 244.0910; IR (neat): 3151, 3112, 2944, 2881, 1514, 1487, 1460, 1409, 1369, 1358, 1297, 1274, 1232, 1097, 1006, 966, 741, $660 \mathrm{~cm}^{-1} ; \mathbf{R}_{\mathbf{f}}(3: 7$ hexanes/EtOAc; $\left.\mathrm{KMnO}_{4}\right): 0.60$.

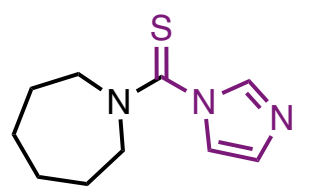

$4 \mathbf{j}$

Azepan-1-yl(1H-imidazol-1-yl)methanethione (4j). Prepared on 2.0-mmol scale following General Procedure F. The crude residue was purified by flash column chromatography (gradient of $20-90 \%$ EtOAc/hexanes $)$ to yield the product as a white solid $(0.37 \mathrm{~g}, 1.8 \mathrm{mmol}, 89 \%) .{ }^{1} \mathbf{H}$ NMR $\left(400 \mathrm{MHz}, \mathrm{CDCl}_{3}, 298 \mathrm{~K}\right): \delta_{\mathrm{H}} 7.77(\mathrm{~s}, 1 \mathrm{H}), 7.15(\mathrm{~d}, J=1.4 \mathrm{~Hz}, 1 \mathrm{H}), 7.03(\mathrm{t}, J=1.2 \mathrm{~Hz}$, 1H), 4.00 (s (br), 2H), 3.55 (s (br), 2H), 2.06-1.44 (m, 8H) ppm. ${ }^{13} \mathbf{C}$ NMR (101 MHz, CDCl 3 , $298 \mathrm{~K}): \delta_{\mathrm{C}} 178.7,136.9,129.5,119.2,55.0,53.5,28.9,27.6,26.2,25.7 \mathrm{ppm}$. HRMS $\mathrm{m} / \mathrm{z}$ (DART): calculated for $\mathrm{C}_{10} \mathrm{H}_{16} \mathrm{~N}_{3} \mathrm{~S}(\mathrm{M}+\mathrm{H}): 210.1059$; found: 210.1063; IR (neat): 3145, 3106, 2943, 2917, 2855, 1607, 1518, 1492, 1422, 1356, 1300, 1283, 1266, 976, 1170, 1092, 1028, 927 , $741,653,601 \mathrm{~cm}^{-1} ; \mathbf{R}_{\mathbf{f}}\left(2: 8\right.$ hexanes/EtOAc; $\left.\mathrm{KMnO}_{4}\right): 0.31$. 
<smiles>CCCSC(=S)n1ccnc1</smiles>

4k

Propyl 1H-imidazole-1-carbodithioate (4k). Prepared on 4.0-mmol scale following General Procedure F. The crude residue was purified by flash column chromatography (gradient of 0 $40 \% \mathrm{EtOAc} / \mathrm{hexanes})$ to yield the product as a dark-yellow oil $(0.59 \mathrm{~g}, 3.2 \mathrm{mmol}, 80 \%) .{ }^{1} \mathbf{H}$ NMR $\left(400 \mathrm{MHz}, \mathrm{CDCl}_{3}, 298 \mathrm{~K}\right): \delta_{\mathrm{H}} 8.49(\mathrm{t}, J=1.1 \mathrm{~Hz}, 1 \mathrm{H}), 7.79(\mathrm{t}, J=1.5 \mathrm{~Hz}, 1 \mathrm{H}), 7.10(\mathrm{dd}, J$ $=1.8,0.9 \mathrm{~Hz}, 1 \mathrm{H}), 3.37(\mathrm{t}, J=7.2 \mathrm{~Hz}, 2 \mathrm{H}), 1.82(\mathrm{sxt}, J=7.4 \mathrm{~Hz}, 2 \mathrm{H}), 1.08(\mathrm{t}, J=7.4 \mathrm{~Hz}, 3 \mathrm{H})$ ppmz. ${ }^{13} \mathbf{C}$ NMR $\left(101 \mathrm{MHz}, \mathrm{CDCl}_{3}, 298 \mathrm{~K}\right): \delta_{\mathrm{C}} 198.5,135.7,131.4,117.9,39.0,21.1,13.7 \mathrm{ppm}$. HRMS $m / z$ (DART): calculated for $\mathrm{C}_{7} \mathrm{H}_{11} \mathrm{~N}_{2} \mathrm{~S}_{2}(\mathrm{M}+\mathrm{H})$ : 187.0370 ; found: 187.0368 ; IR (neat): 3116, 2963, 2930, 2872, 1527, 1461, 1366, 1268, 1217, 1093, 1045, 1003, 829, 742, 721,643 $\mathrm{cm}^{-1} ; \mathbf{R}_{\mathbf{f}}\left(7: 3\right.$ hexanes/EtOAc; $\left.\mathrm{KMnO}_{4}\right): 0.70$.<smiles>S=C(OCCCc1ccccc1)n1ccnc1</smiles>

4!

$O$-(3-Phenylpropyl) $1 \mathrm{H}$-imidazole-1-carbothioate (4I). Prepared on 5.0-mmol scale following General Procedure F with the following modification: the reaction was stirred for 24 hours instead of 2 hours. The crude residue was purified by flash column chromatography (gradient of $0-40 \%$ EtOAc/hexanes) to yield the product as a light-yellow oil (1.2 g, $4.7 \mathrm{mmol}, 94 \%) .{ }^{1} \mathbf{H}$ NMR $\left(400 \mathrm{MHz}, \mathrm{CDCl}_{3}, 298 \mathrm{~K}\right): \delta_{\mathrm{H}} 8.26(\mathrm{t}, J=1.1 \mathrm{~Hz}, 1 \mathrm{H}), 7.57(\mathrm{t}, J=1.5 \mathrm{~Hz}, 1 \mathrm{H}), 7.36-7.27$ $(\mathrm{m}, 2 \mathrm{H}), 7.27-7.16(\mathrm{~m}, 3 \mathrm{H}), 7.03(\mathrm{dd}, J=1.8,0.9 \mathrm{~Hz}, 1 \mathrm{H}), 4.68(\mathrm{t}, J=6.4 \mathrm{~Hz}, 2 \mathrm{H}), 2.80(\mathrm{t}, J=$ $7.5 \mathrm{~Hz}, 2 \mathrm{H}), 2.28-2.17$ (m, 2H) ppm. $\left.{ }^{13} \mathbf{C ~ N M R ~ ( 1 0 1 ~ M H z , ~} \mathrm{CDCl}_{3}, 298 \mathrm{~K}\right): \delta_{\mathrm{C}} 184.3,140.5$, 136.8, 130.7, 128.8, 128.5, 126.5, 118.0, 73.2, 32.4, 29.7 ppm. HRMS $m / z$ (DART): calculated for $\mathrm{C}_{13} \mathrm{H}_{15} \mathrm{~N}_{2} \mathrm{OS}(\mathrm{M}+\mathrm{H})$ : 247.0900; found: 247.0902; IR (neat): 3127, 3026, 2939, 2860, 1739, $1603,1530,1384,1323,1229,1091,993,962,892,743,699,655,583 \mathrm{~cm}^{-1} ; \mathbf{R}_{\mathbf{f}}(9: 1$ hexanes/EtOAc; $\mathrm{KMnO}_{4}$ ): 0.16 .<smiles>S=C(OC1CCCCC1)n1ccnc1</smiles>

$4 \mathrm{~m}$

$\boldsymbol{O}$-Cyclohexyl $\mathbf{1 H}$-imidazole-1-carbothioate (3m). Prepared on 5.0-mmol scale following General Procedure F with the following modification: the reaction was stirred for 24 hours instead of 2 hours. The crude residue was purified by flash column chromatography (gradient of 0-40\% EtOAc/hexanes) to yield the product as a light-yellow oil $(0.79 \mathrm{~g}, 3.9 \mathrm{mmol}, 75 \%) .{ }^{1} \mathbf{H}$ NMR $\left(400 \mathrm{MHz}, \mathrm{CDCl}_{3}, 298 \mathrm{~K}\right): \delta_{\mathrm{H}} 8.36(\mathrm{~d}, J=1.1 \mathrm{~Hz}, 1 \mathrm{H}), 7.64(\mathrm{t}, J=1.5 \mathrm{~Hz}, 1 \mathrm{H}), 7.03(\mathrm{dd}$, $J=1.8,0.9 \mathrm{~Hz}, 1 \mathrm{H}), 5.51(\mathrm{tt}, J=8.5,3.8 \mathrm{~Hz}, 1 \mathrm{H}), 2.10-1.99(\mathrm{~m}, 2 \mathrm{H}), 1.85-1.65(\mathrm{~m}, 4 \mathrm{H}), 1.63-$ $1.56(\mathrm{~m}, 1 \mathrm{H}), 1.56-1.34(\mathrm{~m}, 3 \mathrm{H}) \mathrm{ppm} .{ }^{13} \mathrm{C}$ NMR (101 MHz, $\left.\mathrm{CDCl}_{3}, 298 \mathrm{~K}\right): \delta_{\mathrm{C}} 183.4,136.8$, 130.6, 118.0, 82.8, 30.8, 25.3, $23.6 \mathrm{ppm}$. HRMS $\mathrm{m} / \mathrm{z}$ (DART): calculated for $\mathrm{C}_{10} \mathrm{H}_{15} \mathrm{~N}_{2} \mathrm{OS}$ 
(M+H): 211.0900; found: 211.0901; IR (neat): 3127, 2933, 2858, 1738, 1529, 1460, 1380, 1322, $1380,1227,1096,967,886,742,656 \mathrm{~cm}^{-1} ; \mathbf{R}_{\mathbf{f}}\left(9: 1\right.$ hexanes/EtOAc; $\left.\mathrm{KMnO}_{4}\right): 0.29$.<smiles>COC(=O)C([14CH3])OC(=S)n1ccnc1</smiles>

$4 n$

Methyl 2-((1H-imidazole-1-carbonothioyl)oxy)propanoate (3n). Prepared on 5.0-mmol scale following General Procedure F with the following modification: the reaction was stirred for 24 hours instead of 2 hours. The crude residue was purified by flash column chromatography (gradient of 0-30\% EtOAc/hexanes) to yield the product as a light-yellow oil $(1.1 \mathrm{~g}, 4.6 \mathrm{mmol}$, 92\%). ${ }^{1} \mathbf{H}$ NMR $\left(400 \mathrm{MHz}, \mathrm{CDCl}_{3}, 298 \mathrm{~K}\right): \delta_{\mathrm{H}} 8.37(\mathrm{p}, J=1.0 \mathrm{~Hz}, 1 \mathrm{H}), 7.66(\mathrm{td}, J=1.7,0.8 \mathrm{~Hz}$, $1 \mathrm{H}), 7.05(\mathrm{dq}, J=1.8,0.9 \mathrm{~Hz}, 1 \mathrm{H}), 5.75(\mathrm{q}, J=6.5 \mathrm{~Hz}, 1 \mathrm{H}), 3.79(\mathrm{~s}, 3 \mathrm{H}), 1.72(\mathrm{~d}, J=7.0 \mathrm{~Hz}$, 3H) ppm. ${ }^{13}$ C NMR (101 MHz, $\left.\mathrm{CDCl}_{3}, 298 \mathrm{~K}\right): \delta_{\mathrm{C}} 183.4,169.6,137.1,131.2,118.2,76.3,52.9$, $17.1 \mathrm{ppm}$. HRMS $m / z$ (DART): calculated for $\mathrm{C}_{9} \mathrm{H}_{14} \mathrm{~N}_{2} \mathrm{O}_{3} \mathrm{~S}$ (M+H): 229.0641; found: 229.0645; IR (neat): 3134, 2984. 2940. 2906, 1747, 1532, 1466, 1390, 1328, 1282, 1209, 1132, 1081, 967, $840,743,654 \mathrm{~cm}^{-1} ; \mathbf{R}_{\mathbf{f}}\left(7: 3\right.$ hexanes/EtOAc; UV, $\left.\mathrm{KMnO}_{4}\right): 0.35$. 


\section{One-pot and telescoped reactions}

Thioamide:

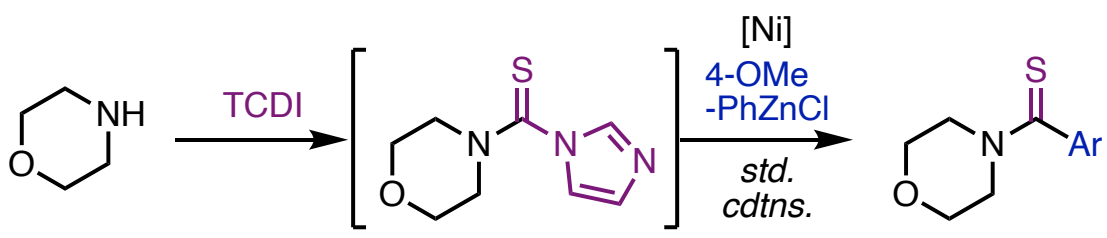

$7 a$

$4 e$

5e, $52 \%$ (one-pot)

telescoped: $81 \%$

One-pot procedure:

To a flame-dried 16-mL reaction tube with a stir bar was added $7 \mathbf{a}(26 \mu \mathrm{L}, 0.30 \mathrm{mmol}$, 1.0 equiv.). The flask was sealed, evacuated and backfilled with $\mathrm{N}_{2}(\times 3)$, and 1,4-dioxane (anhydrous, degassed for 15 minutes by sparging with argon; $2.0 \mathrm{~mL}$ ) was added. While the mixture was stirring, the flask was briefly opened to air and 1,1'-thiocarbonyldiimidazole (56 $\mathrm{mg}, 0.32 \mathrm{mmol}, 1.1$ equiv.) was added. The flask was sealed, wrapped in aluminum foil, and stirred at r.t. for $2 \mathrm{~h}$. To a flame-dried 5-mL vial was added $\mathrm{Ni}(\mathrm{acac})_{2} \bullet \mathrm{xH}_{2} \mathrm{O}(1.5 \mathrm{mg}, 0.060$ $\mathrm{mmol}, 2.0 \mathrm{~mol} \%$ ), and phenanthroline $(2.7 \mathrm{mg}, 0.015 \mathrm{mmol}, 5.0 \mathrm{~mol} \%)$. The vial was sealed with a septum, and evacuated and backfilled with $\mathrm{N}_{2}(\times 3)$. 1,4-Dioxane (anhydrous, degassed for 15 minutes by sparging with argon; $1 \mathrm{~mL}$ ) was added and the mixture was stirred at r.t. for 10 min. After $10 \mathrm{~min}$., the solution was transferred to the $16-\mathrm{mL}$ reaction tube containing the reaction mixture. While stirring, $4-\mathrm{OMePhZnCl}$ solution $(1.3 \mathrm{~mL}$ of a $0.36 \mathrm{M}$ solution in $\mathrm{THF}$, $0.45 \mathrm{mmol}, 3.0$ equiv.) was added at once and the reaction was stirred at r.t. $\left(23^{\circ} \mathrm{C}\right)$ for $1 \mathrm{~h}$. The reaction was opened to air and quenched with sat. aq. $\mathrm{NH}_{4} \mathrm{Cl}(\sim 1.5 \mathrm{~mL})$. EtOAc $(\sim 2 \mathrm{~mL})$ was added, and the reaction mixture was mixed vigorously through pipetting. The organic layer was filtered through a Pasteur pipette loaded with $\mathrm{MgSO}_{4}$ and celite. An additional $2 \mathrm{~mL}$ of EtOAc was added to the remaining aqueous layer, vigorously mixed, and the organic fraction was passed through the same $\mathrm{MgSO}_{4} /$ celite plug. (The sulfur-containing aqueous waste was treated with bleach and disposed of appropriately. The crude residue was purified by flash column chromatography (gradient of $0-30 \%$ EtOAc/hexanes) to yield the product 5e as a yellow solid (37 mg, $0.16 \mathrm{mmol}, 52 \%$ ).

\section{Telescoped procedure:}

To a flame-dried $10-\mathrm{mL}$ round bottom flask with stir bar was added $7 \mathbf{a}(26 \mu \mathrm{L}, 0.30$ mmol, 1.0 equiv.). The flask was sealed, evacuated and backfilled with $\mathrm{N}_{2}(\times 3)$, and dry $\mathrm{CH}_{2} \mathrm{Cl}_{2}$ $(2.0 \mathrm{~mL})$ was added. While the solution was stirring, the flask was briefly opened to air and 1,1'thiocarbonyldiimidazole (56 mg, $0.32 \mathrm{mmol}, 1.1$ equiv.) was added. The tube was sealed, wrapped in aluminum foil, and stirred at r.t. for $2 \mathrm{~h}$. The reaction was then opened to air, quenched with sat. aq. $\mathrm{NH}_{4} \mathrm{Cl}$, and extracted with $\mathrm{CH}_{2} \mathrm{Cl}_{2}(\times 3)$. The organic fractions were combined, dried over $\mathrm{MgSO}_{4}$, and concentrated to yield the crude material containing the thiocarbonyl imidazolide substrate 4e. 
To a flame-dried 16-mL reaction tube with a stir bar was added $\mathrm{Ni}(\text { acac })_{2} \bullet \mathrm{xH}_{2} \mathrm{O}(1.5 \mathrm{mg}$, $0.060 \mathrm{mmol}, 2.0 \mathrm{~mol} \%$ ), and phenanthroline ( $2.7 \mathrm{mg}, 0.015 \mathrm{mmol}, 5.0 \mathrm{~mol} \%)$. The flask was sealed with a septum and evacuated and backfilled with $\mathrm{N}_{2}(\times 3)$. The crude $O$-thiocarbamate substrate 4e was dissolved in 1,4-dioxane (anhydrous, degassed for 15 minutes by sparging with argon; $2.4 \mathrm{~mL}$ ) and transferred to the $16-\mathrm{mL}$ reaction tube. The reaction mixture was stirred at r.t. for $10 \mathrm{~min}$. After $10 \mathrm{~min}$, while stirring, 4-OMePhZnCl solution $(1.2 \mathrm{~mL}$ of a $0.38 \mathrm{M}$ solution in THF, $0.45 \mathrm{mmol}, 3.0$ equiv.) was added at once and the reaction was stirred at r.t. $\left(23{ }^{\circ} \mathrm{C}\right)$ for $1 \mathrm{~h}$. The reaction was opened to air and quenched with sat. aq. $\mathrm{NH}_{4} \mathrm{Cl}(\sim 1.5 \mathrm{~mL})$. EtOAc $(\sim 2$ $\mathrm{mL}$ ) was added, and the reaction mixture was mixed vigorously through pipetting. The organic layer was filtered through a Pasteur pipette loaded with $\mathrm{MgSO}_{4}$ and celite. An additional $2 \mathrm{~mL}$ of EtOAc was added to the remaining aqueous layer, vigorously mixed, and the organic fraction was passed through the same $\mathrm{MgSO}_{4} /$ celite plug. (The sulfur-containing aqueous waste was treated with bleach and disposed of appropriately.) The combined organic phase was concentrated and the crude residue was purified by flash column chromatography (gradient of 0$30 \% \mathrm{EtOAc} / \mathrm{hexanes})$ to yield the product $5 \mathbf{e}$ as a yellow solid (58 $\mathrm{mg}, 0.24 \mathrm{mmol}, 81 \%$ ).

\section{Thionoester:}

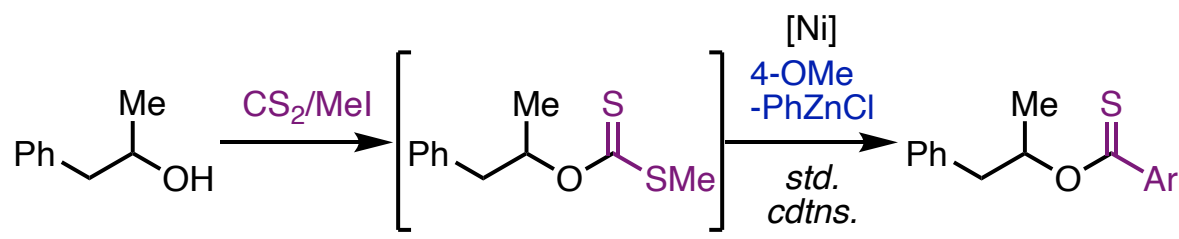

$7 b$

$1 \mathrm{c}$

$3 c, 28 \%$ (one-pot)

telescoped: $35 \%$

One-pot procedure: $:^{2,3}$

To an 8 -mL reaction tube with stir bar was added $7 \mathbf{b}(42 \mu \mathrm{L}, 0.30 \mathrm{mmol}, 1.0$ equiv.). The flask was sealed, evacuated and backfilled with $\mathrm{N}_{2}(\times 3)$, and tetrabutylammonium hydroxide 30hydrate solution $\left(0.1 \mathrm{~mL}\right.$ of a $40 \mathrm{w} / \mathrm{v}$ in $\mathrm{H}_{2} \mathrm{O}$ solution, $0.15 \mathrm{mmol}, 0.5$ equiv. $), \mathrm{CS}_{2}(0.5 \mathrm{~mL})$, and $\mathrm{NaOH}\left(0.5 \mathrm{~mL}\right.$ of a $50 \% \mathrm{w} / \mathrm{v}$ solution in $\left.\mathrm{H}_{2} \mathrm{O}\right)$ were added. The reaction mixture was stirred vigorously for 5 minutes. Iodomethane $(22 \mu \mathrm{L}, 0.36 \mathrm{mmol}, 1.2$ equiv.) was then added dropwise and the reaction mixture was stirred for another 5 minutes. The reaction was opened to air and 1 $\mathrm{mL}$ of $\mathrm{Et}_{2} \mathrm{O}$ was added. After mixing, the aqueous layer was removed by pipetting and the volatile was removed under vacuo to yield the crude material containing crude $O$-alkyl xanthate substrate 1c.

To the 8-mL reaction flask was added bar was added $\mathrm{Ni}(\mathrm{acac})_{2} \bullet \mathrm{xH}_{2} \mathrm{O}(1.5 \mathrm{mg}, 0.060$ mmol, $2.0 \mathrm{~mol} \%$ ), and bathocuproine ( $5.4 \mathrm{mg}, 0.015 \mathrm{mmol}, 5.0 \mathrm{~mol} \%$ ). The flask was sealed with a septum, and evacuated and backfilled with $\mathrm{N}_{2}(\times 3)$. 1,4-Dioxane (anhydrous, degassed for 15 minutes by sparging with argon; $2.6 \mathrm{~mL}$ ) was added and the reaction mixture was stirred at r.t. for $10 \mathrm{~min}$. After $10 \mathrm{~min}$, while stirring, $4-\mathrm{OMePhZnCl}$ solution $(1.3 \mathrm{~mL}$ of a $0.36 \mathrm{M}$ solution in THF, $0.45 \mathrm{mmol}, 3.0$ equiv.) was added at once and the reaction was stirred at r.t. $\left(23{ }^{\circ} \mathrm{C}\right)$ for 5 minutes. The reaction was opened to air and quenched with sat. aq. $\mathrm{NH}_{4} \mathrm{Cl}(\sim 1.5 \mathrm{~mL})$. EtOAc $(\sim 2 \mathrm{~mL})$ was added, and the reaction mixture was mixed vigorously through pipetting. The organic layer was filtered through a Pasteur pipette loaded with $\mathrm{MgSO}_{4}$ and celite. An additional 
$2 \mathrm{~mL}$ of EtOAc was added to the remaining aqueous layer, the solution was vigorously mixed, and the organic fraction was passed through the same $\mathrm{MgSO}_{4} /$ celite plug. (The sulfur-containing aqueous waste was treated with bleach and disposed of appropriately.) The combined organic phase was concentrated, and the crude residue was purified by flash column chromatography (gradient of $0-60 \% \mathrm{PhMe} /$ hexanes) to yield the product $3 \mathrm{c}$ as a yellow oil (24 $\mathrm{mg}, 0.084 \mathrm{mmol}$, $28 \%)$.

\section{Telescoped procedure:}

To a flame-dried 10-mL round bottom flask with stir bar was added $7 \mathbf{b}(42 \mu \mathrm{L}, 0.30$ mmol, 1.0 equiv.). The flask was sealed, evacuated and backfilled with $\mathrm{N}_{2}(\times 3)$. THF $(1 \mathrm{~mL})$ was added and the solution was cooled to $0{ }^{\circ} \mathrm{C}$ while stirring. $\mathrm{CS}_{2}(20 \mu \mathrm{L}, 0.33 \mathrm{mmol}, 1.1$ equiv $)$ was added, followed by sodium hydride (13 $\mathrm{mg}$ of a $60 \% \mathrm{w} / \mathrm{w}$ dispersion in mineral oil, $0.33 \mathrm{mmol}$, 1.1 equiv), and the reaction was allowed to warm to r.t. while stirring over $1 \mathrm{~h}$. The reaction was cooled to $0{ }^{\circ} \mathrm{C}$ and iodomethane $(22 \mu \mathrm{L}, 0.36 \mathrm{mmol}, 1.2$ equiv.) was added. The reaction was allowed to warm to r.t. while stirring over $1 \mathrm{~h}$. The reaction was quenched with sat. aq. $\mathrm{NH}_{4} \mathrm{Cl}$ and extracted with EtOAc $(\times 3)$. The organic fractions were combined, dried over $\mathrm{MgSO}_{4}$, and concentrated to yield the crude material containing crude $O$-alkyl xanthate substrate 1 c.

To a flame-dried 8-mL reaction tube with a stir bar was added $\mathrm{Ni}(\mathrm{acac})_{2} \bullet \mathrm{xH}_{2} \mathrm{O}(1.5 \mathrm{mg}$, $0.060 \mathrm{mmol}, 2.0 \mathrm{~mol} \%$ ), and bathocuproine (5.4 mg, $0.015 \mathrm{mmol}, 5.0 \mathrm{~mol} \%$ ). The flask was sealed with a septum and evacuated and backfilled with $\mathrm{N}_{2}(\times 3)$. The crude $O$-alkyl xanthate substrate 1c was dissolved in 1,4-dioxane (anhydrous, degassed for 15 minutes by sparging with argon; $2.4 \mathrm{~mL}$ ) and transferred to the $8-\mathrm{mL}$ reaction tube. The reaction mixture was stirred at r.t. for $10 \mathrm{~min}$. After $10 \mathrm{~min}$, while stirring, $4-\mathrm{OMePhZnCl}$ solution $(1.2 \mathrm{~mL}$ of a $0.38 \mathrm{M}$ solution in THF, $0.45 \mathrm{mmol}, 3.0$ equiv.) was added at once and the reaction was stirred at r.t. $\left(23{ }^{\circ} \mathrm{C}\right)$ for 5 minutes. The reaction was opened to air and quenched with sat. aq. $\mathrm{NH}_{4} \mathrm{Cl}(\sim 1.5 \mathrm{~mL})$. EtOAc $(\sim 2 \mathrm{~mL})$ was added, and the reaction mixture was mixed vigorously through pipetting. The organic layer was filtered through a Pasteur pipette loaded with $\mathrm{MgSO}_{4}$ and celite. An additional $2 \mathrm{~mL}$ of EtOAc was added to the remaining aqueous layer, vigorously mixed, and the organic fraction was passed through the same $\mathrm{MgSO}_{4} /$ celite plug. (The sulfur-containing aqueous waste was treated with bleach and disposed of appropriately.) The combined organic phase was concentrated, and the crude residue was purified by flash column chromatography (gradient of $0-60 \% \mathrm{PhMe} /$ hexanes $)$ to yield the product $3 \mathrm{c}$ as a yellow oil (32 $\mathrm{mg}, 0.011 \mathrm{mmol}, 35 \%)$. 


\section{$\underline{\text { J. References }}$}

(1) Krasovskiy, A.; Knochel, P. Convenient Titration Method for Organometallic Zinc, Magnesium, and Lanthanide Reagents. Synthesis. 2006, 5, 890-891.

(2) Lee, A. W. M.; Chan, W. H.; Wong, H. C.; Wong, M. S. One Pot Phase Transfer Synthesis of O-Alkyl, S-Methyl Dithiocarbonates (Xanthates). Synth. Commun. 1989, 19, 547-552.

(3) Cai, A.; Yan, W.; Liu, W. Aryl Radical Activation of C-O Bonds: Copper-Catalyzed Deoxygenative Difluoromethylation of Alcohols. J. Am. Chem. Soc. 2021, 143, 99529960. 

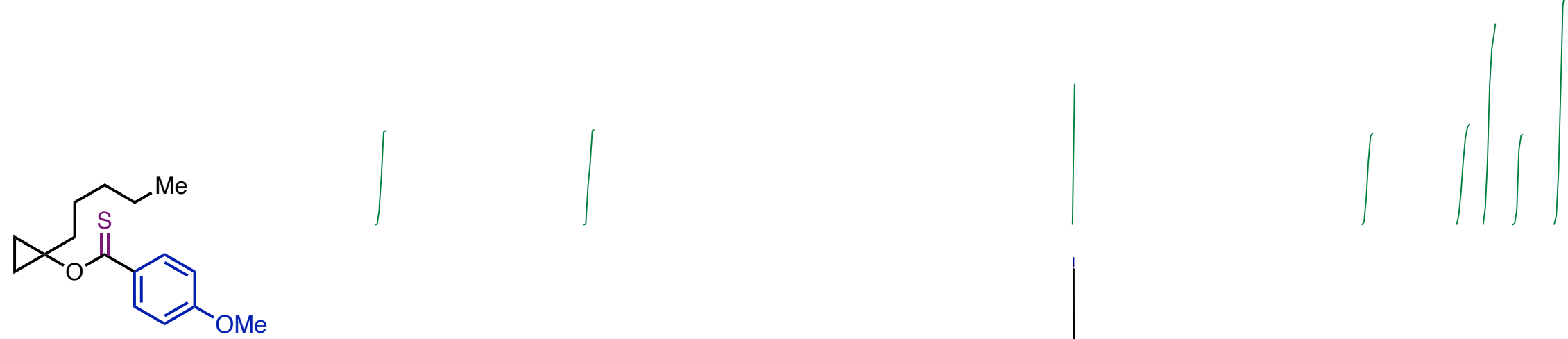

$3 a$

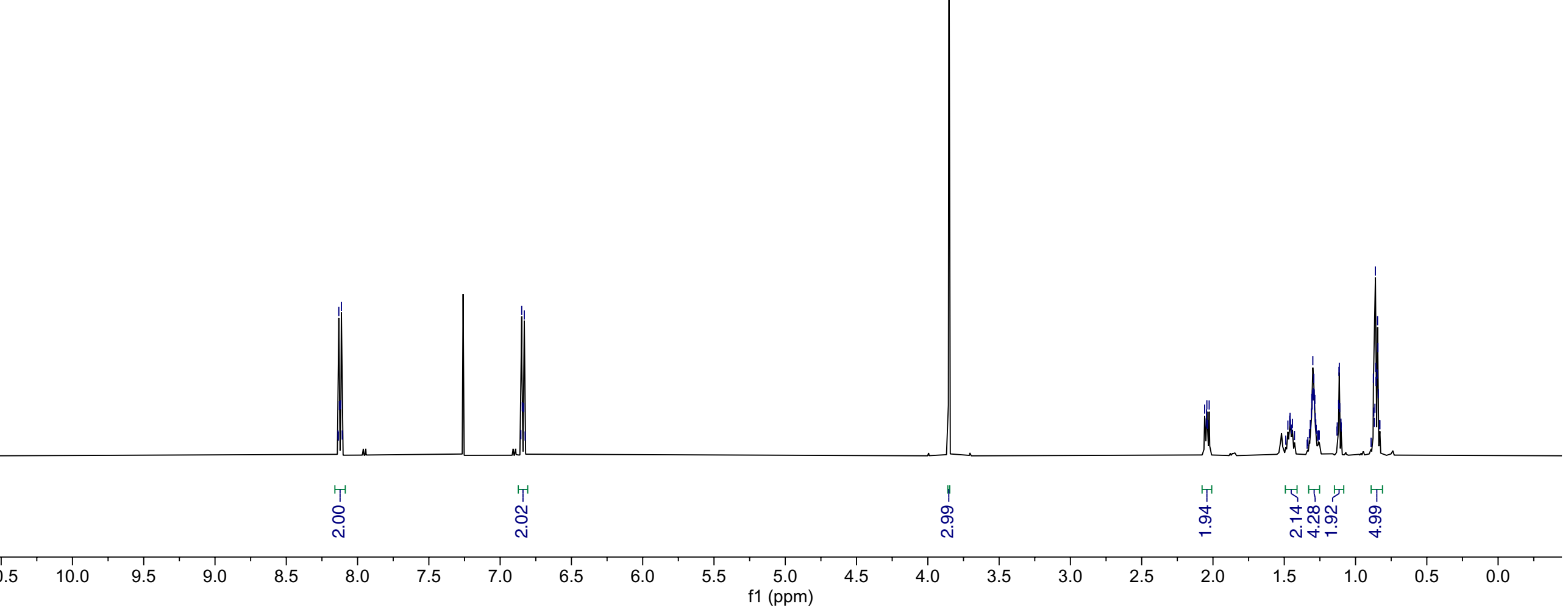




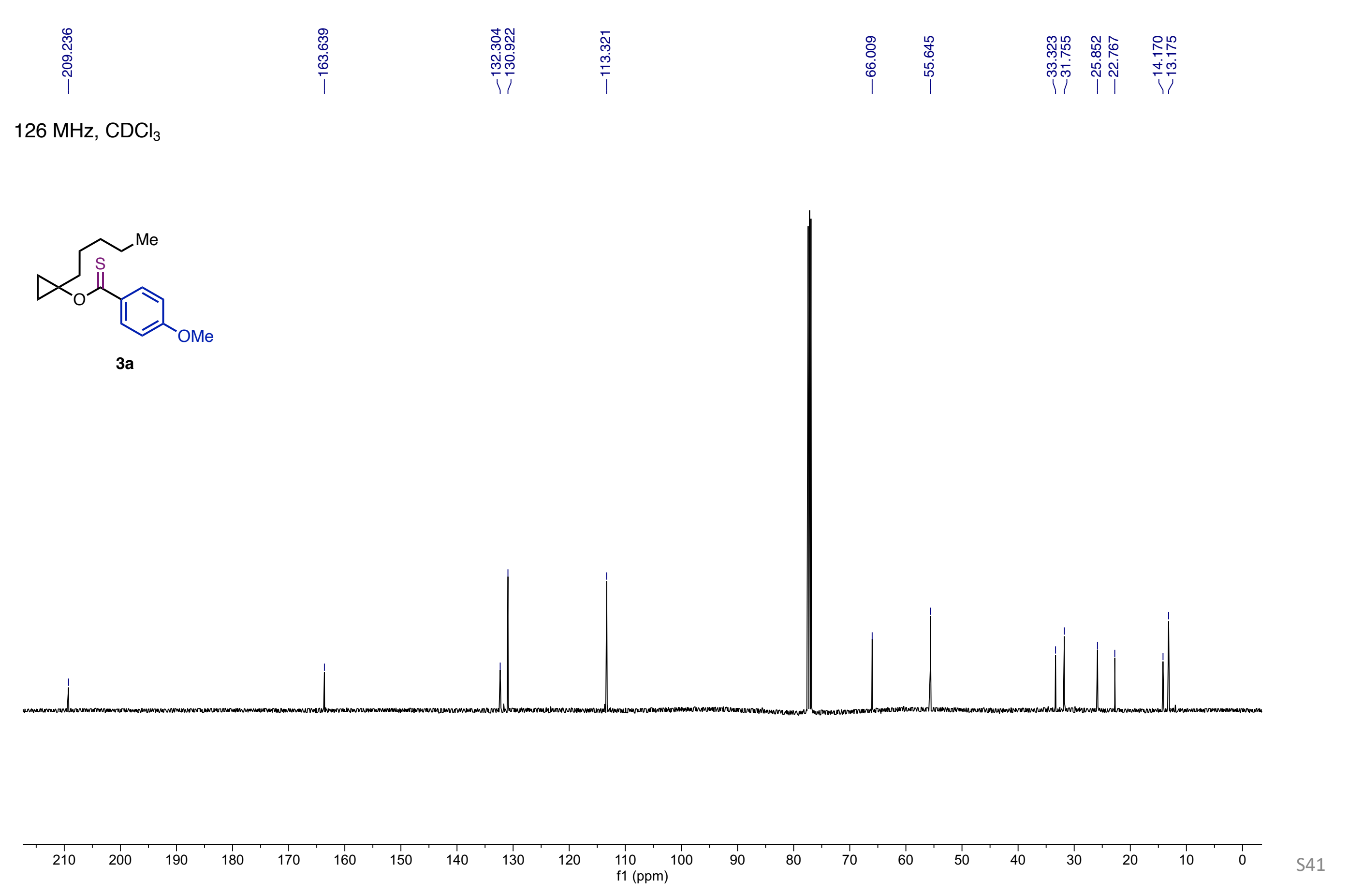



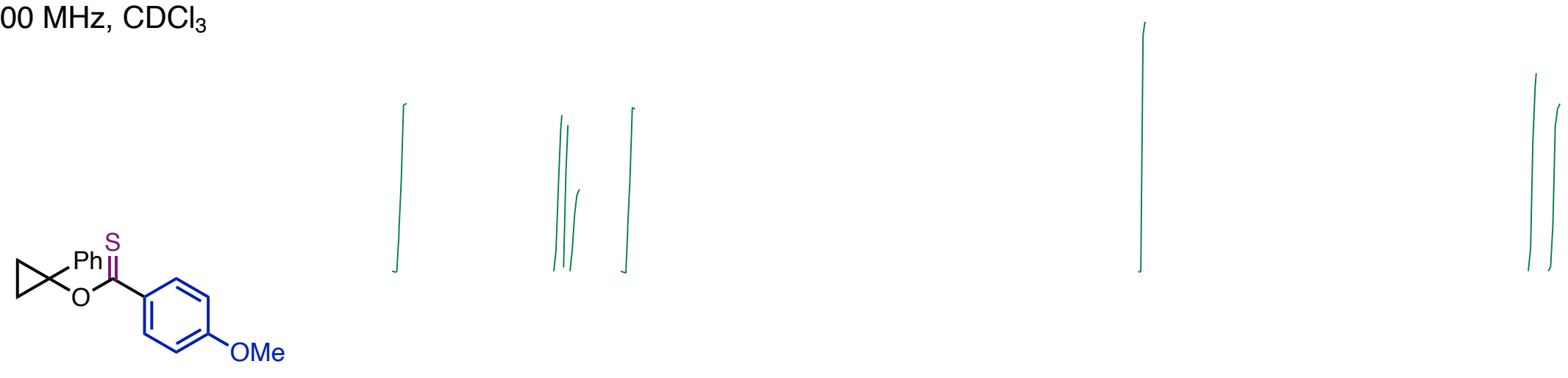

3b

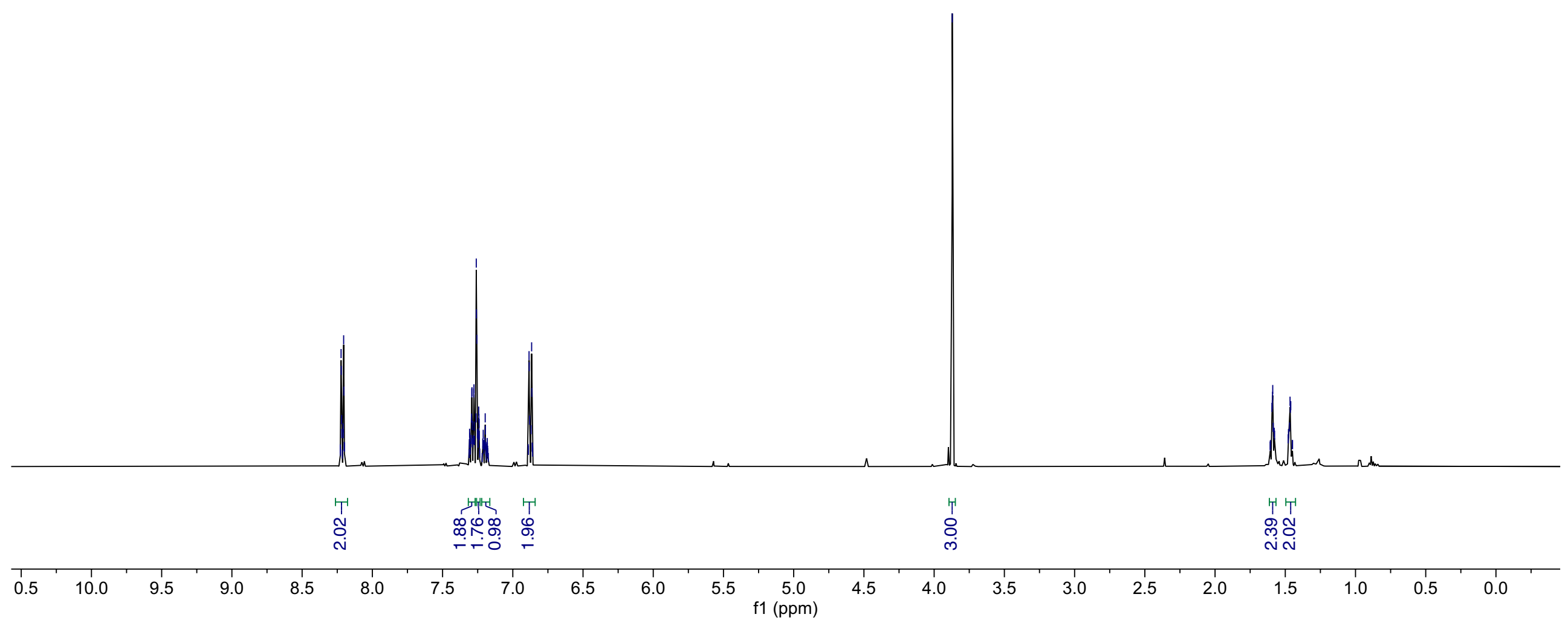


$500 \mathrm{MHz}, \mathrm{CDCl}_{3}$
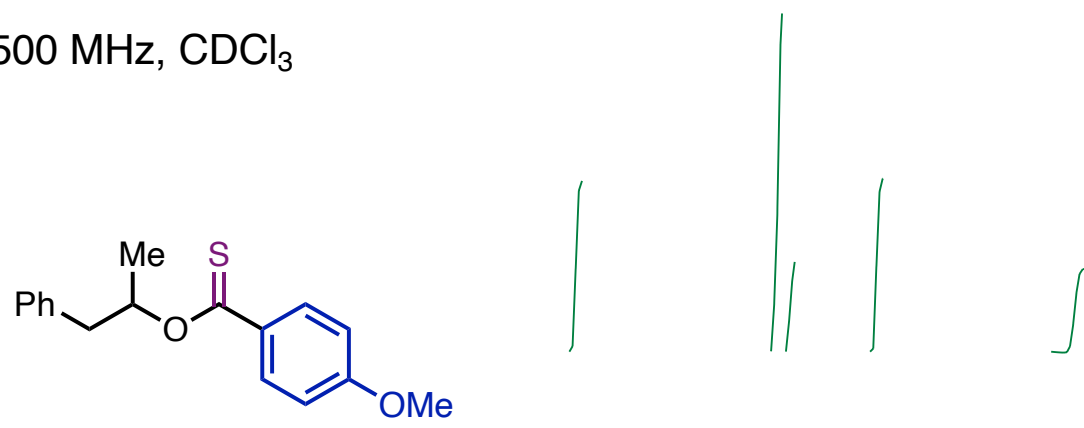

అ

$3 c$
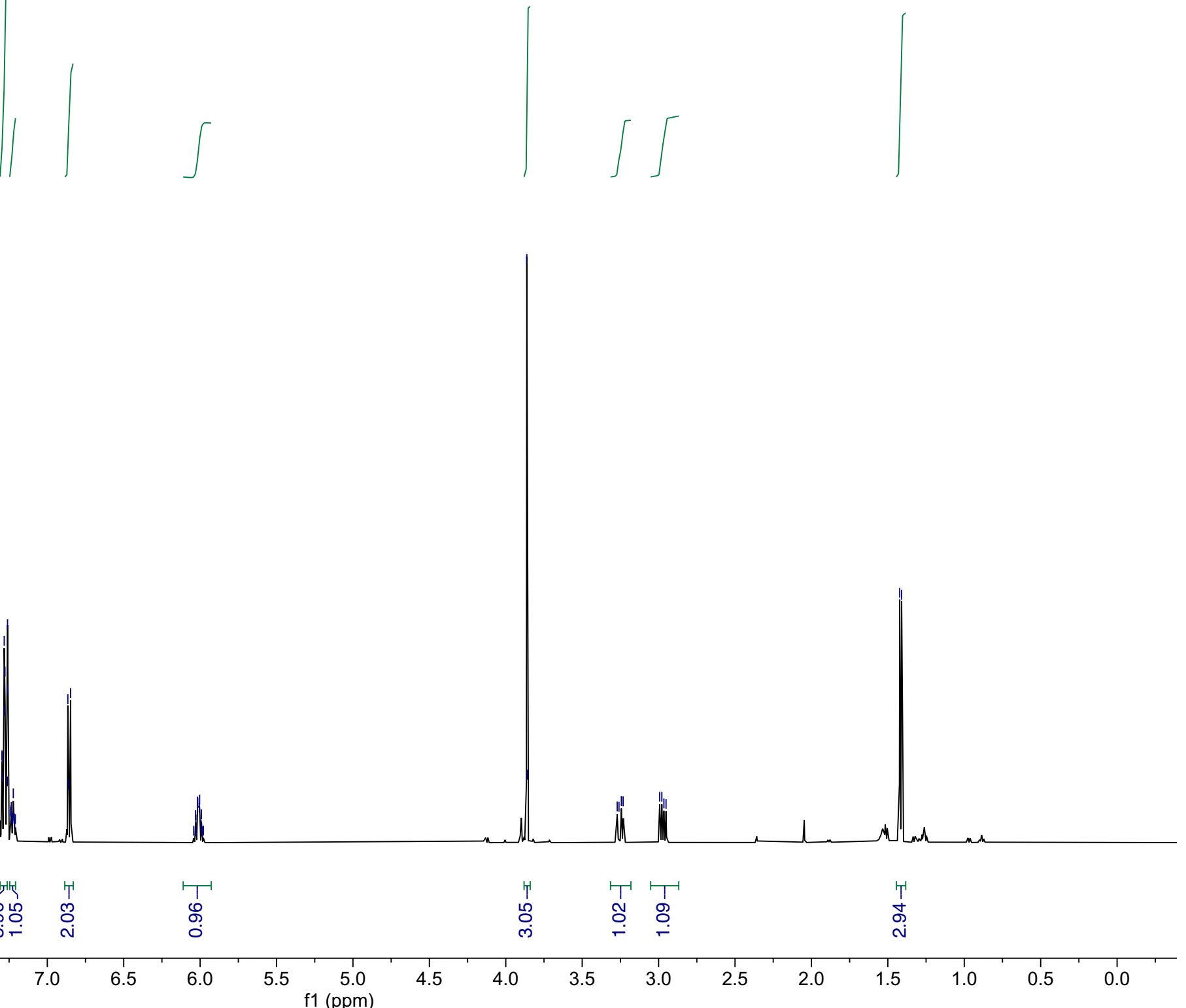
व

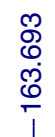

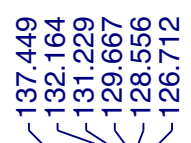

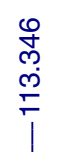

8
$\infty$
$\infty$
$\infty$
1

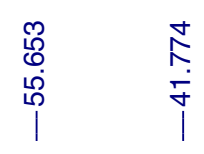

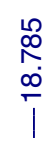

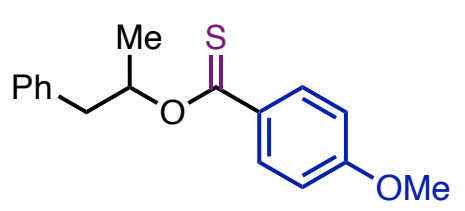

3c

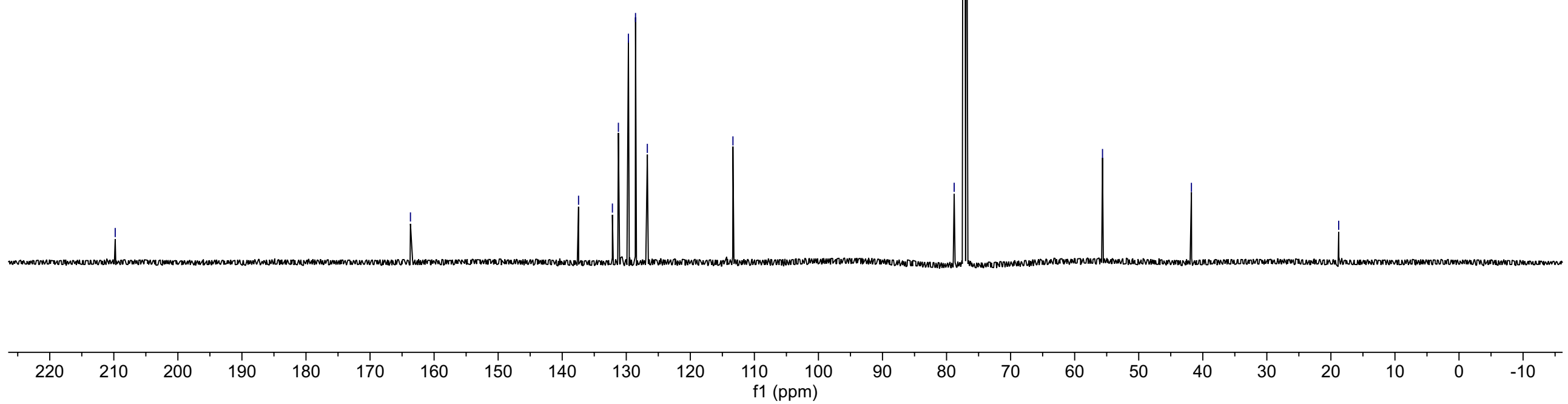

S45 
$500 \mathrm{MHz}, \mathrm{CDCl}_{3}$
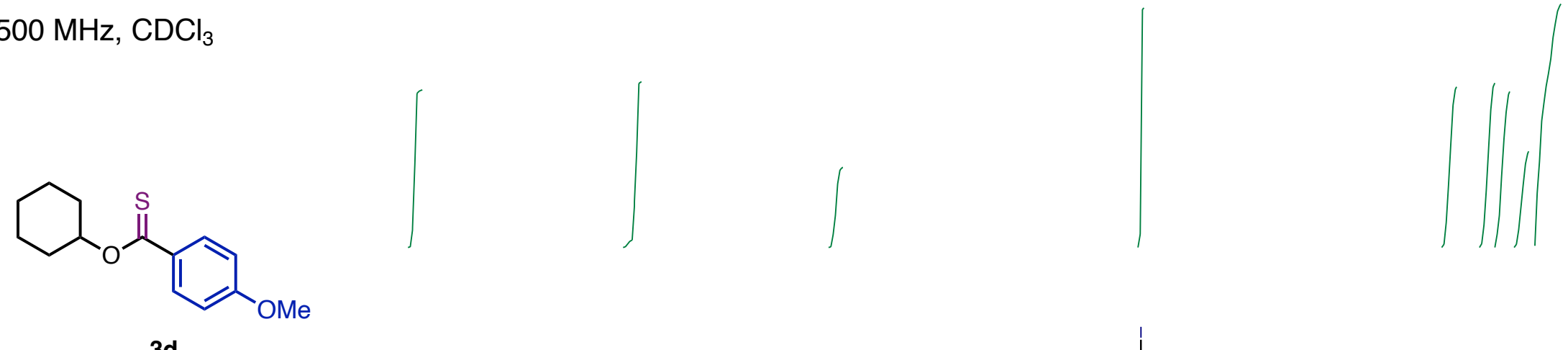

\section{Nhather}

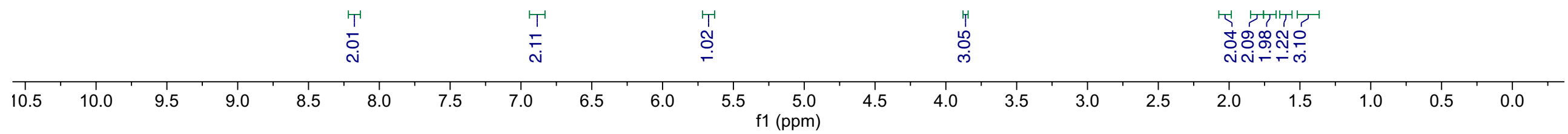




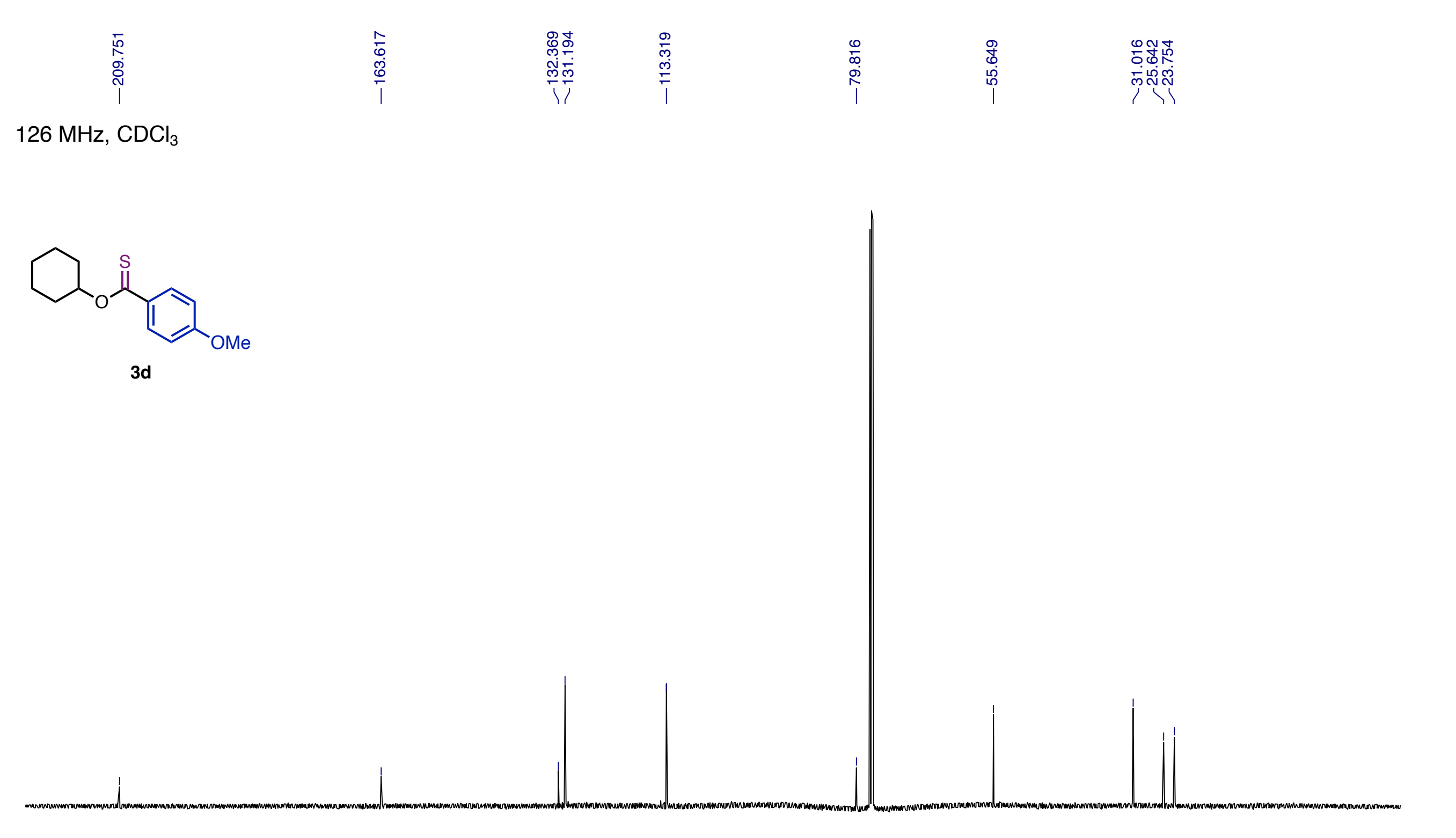


$500 \mathrm{MHz}, \mathrm{CDCl}_{3}$
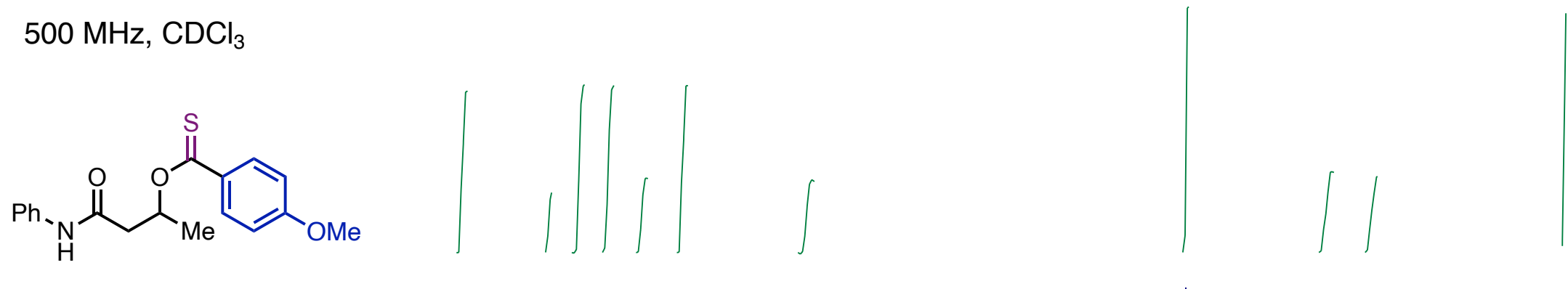

$3 e$

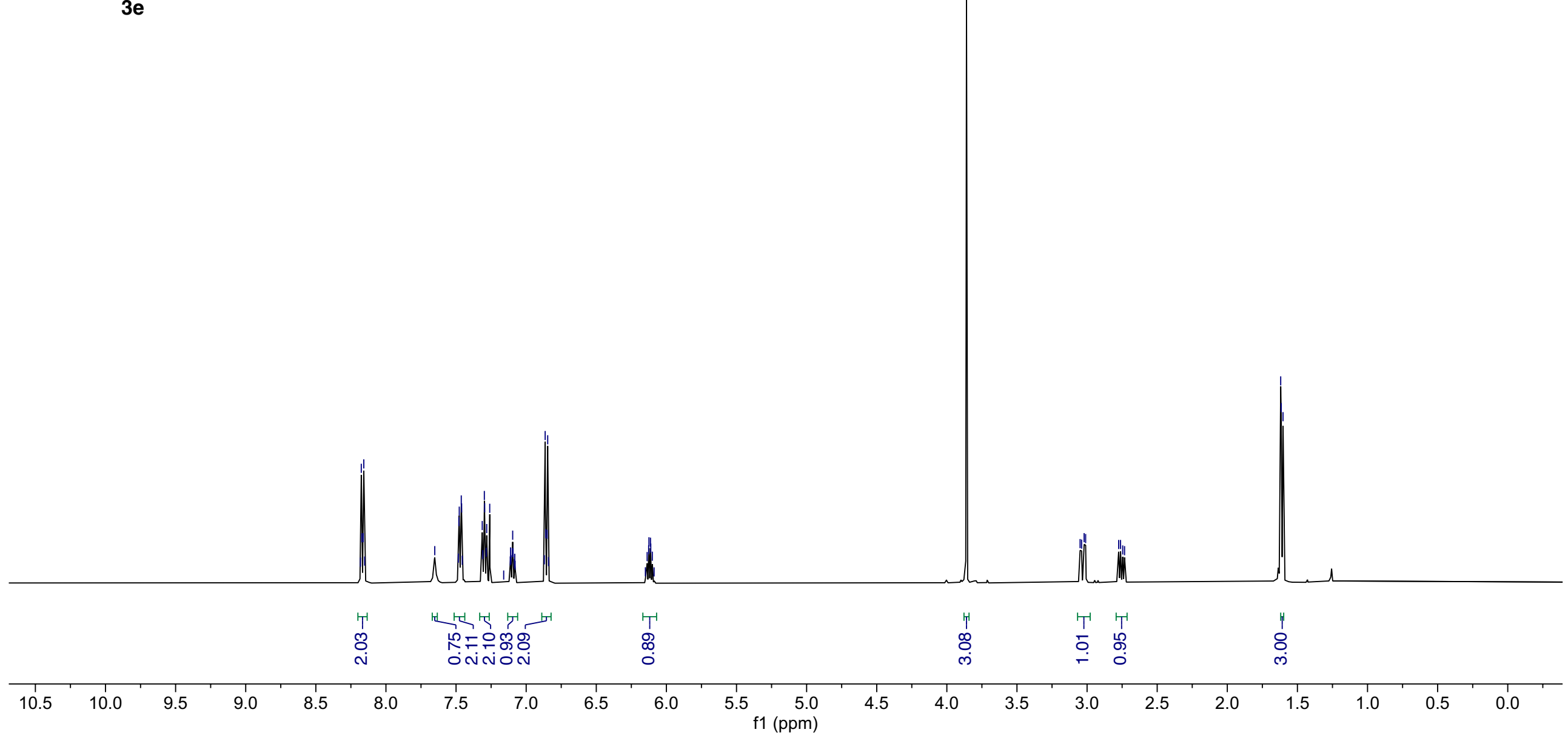




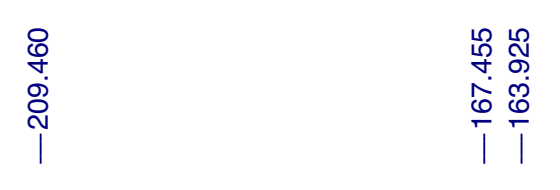

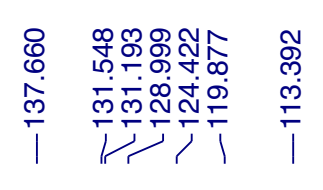

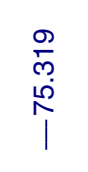

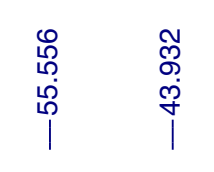

$\hat{o}$
$\dot{q}$
$\stackrel{0}{I}$

$126 \mathrm{MHz}, \mathrm{CDCl}_{3}$<smiles>COc1ccc(C(=S)OC(C)CC(=O)Nc2ccccc2)cc1</smiles>

$3 e$

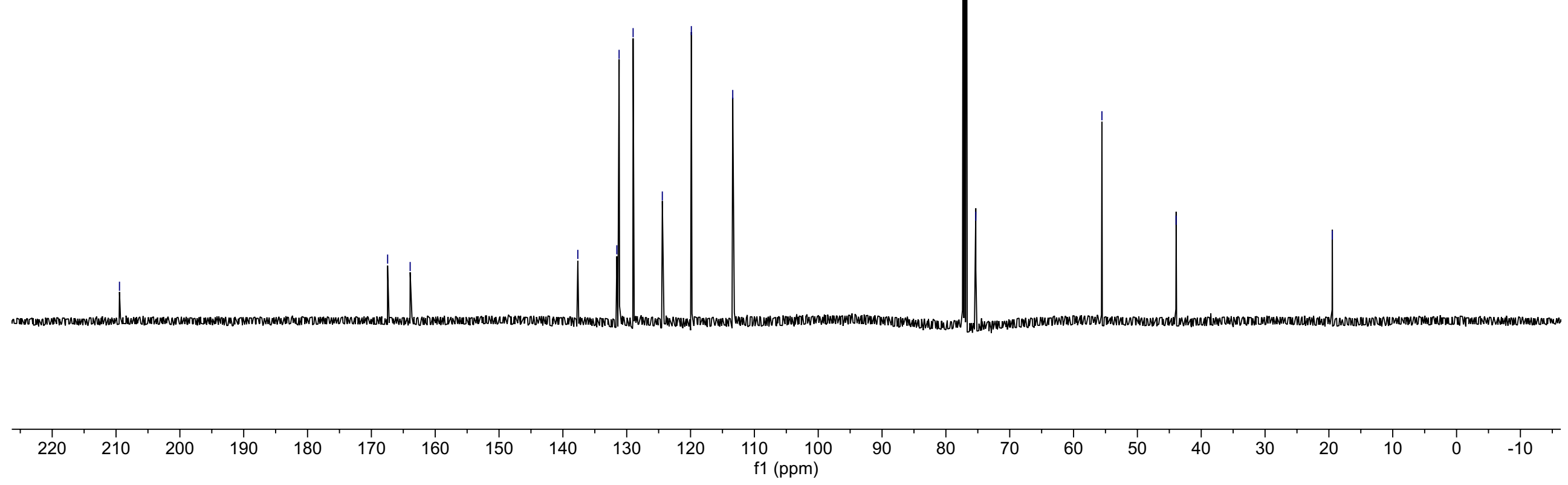

S49 
$500 \mathrm{MHz}, \mathrm{CDCl}_{3}$
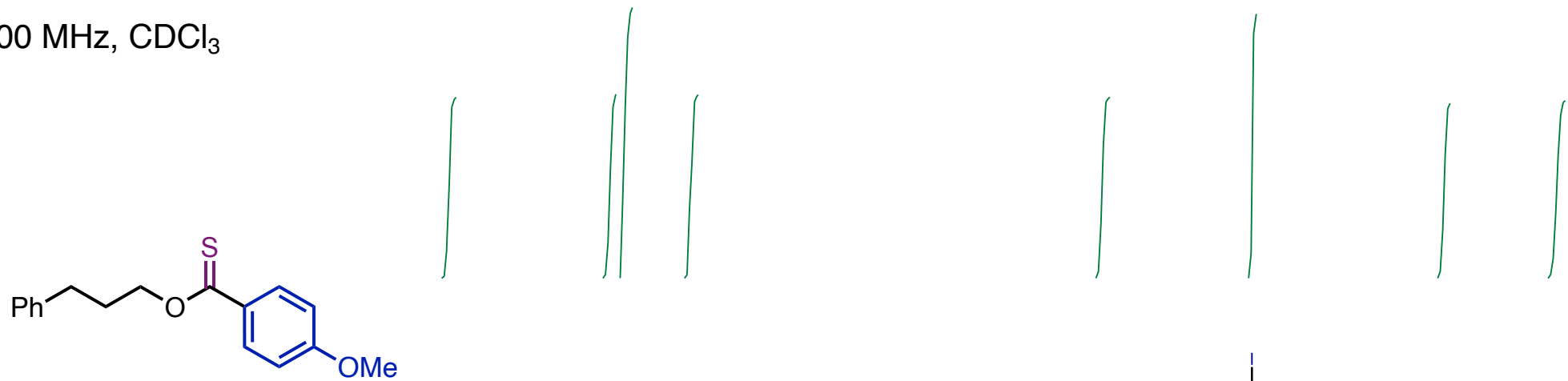

3f
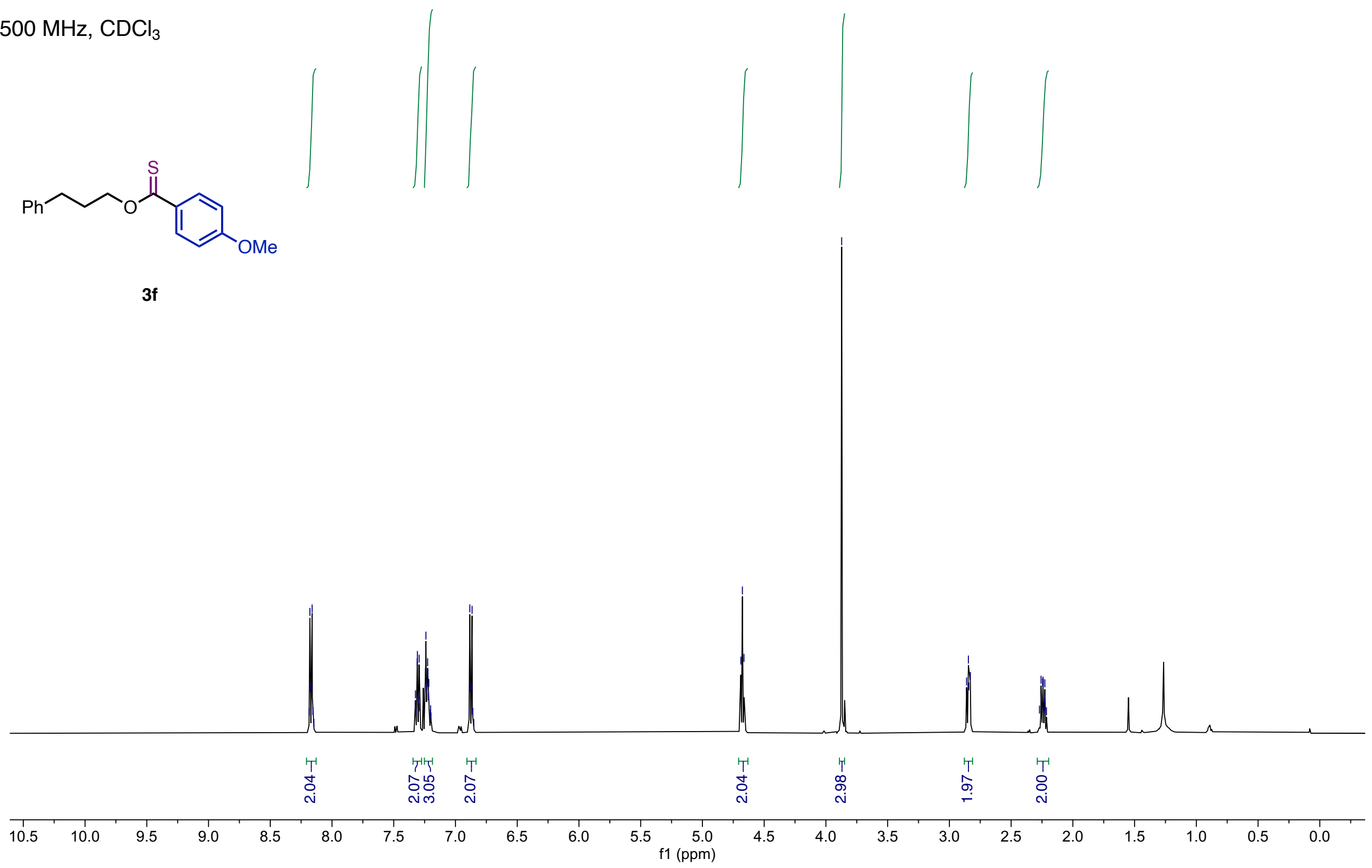
$126 \mathrm{MHz}, \mathrm{CDCl}_{3}$

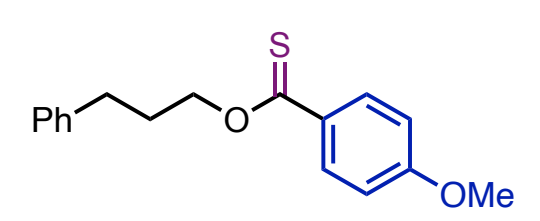

3f

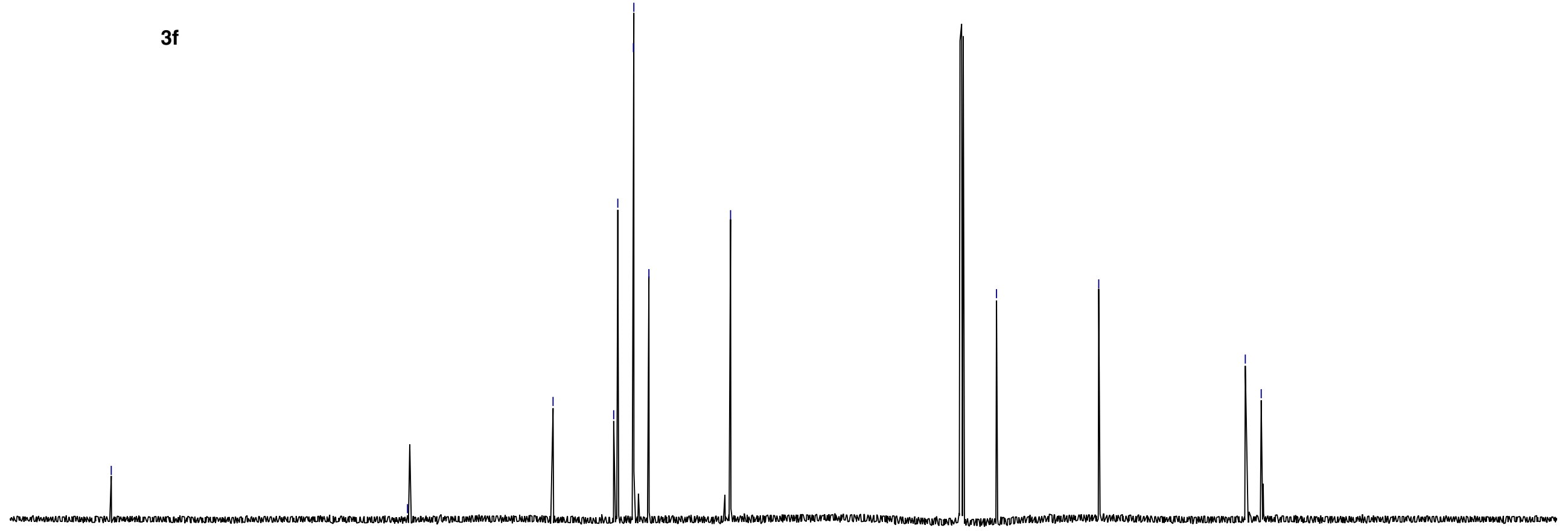



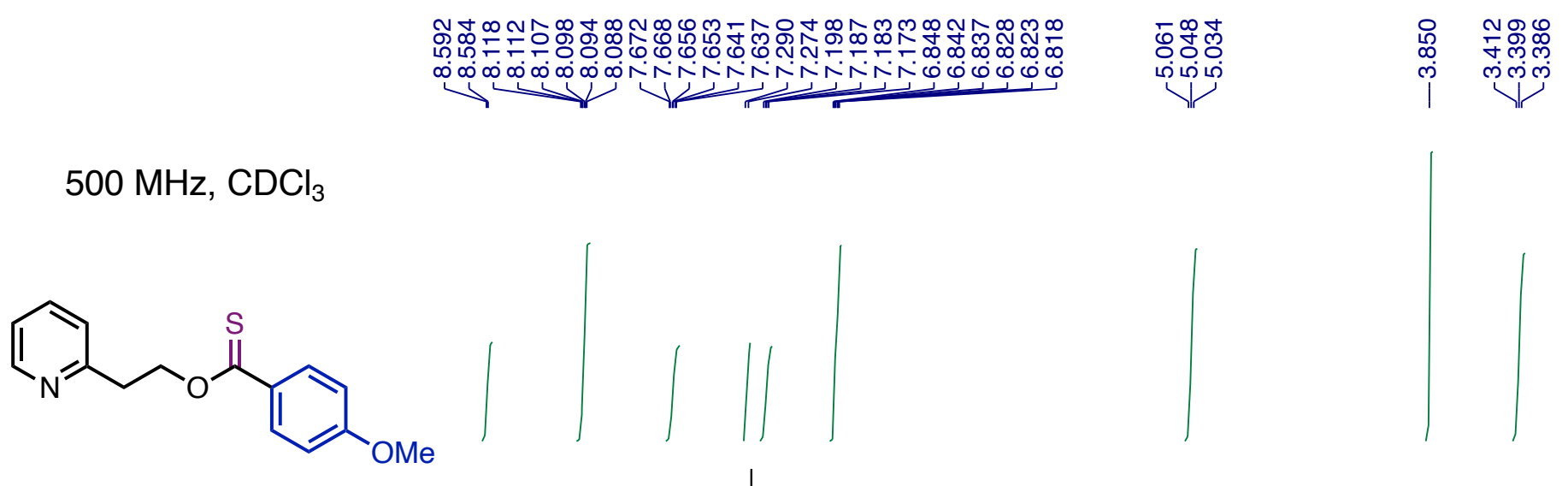

$3 g$
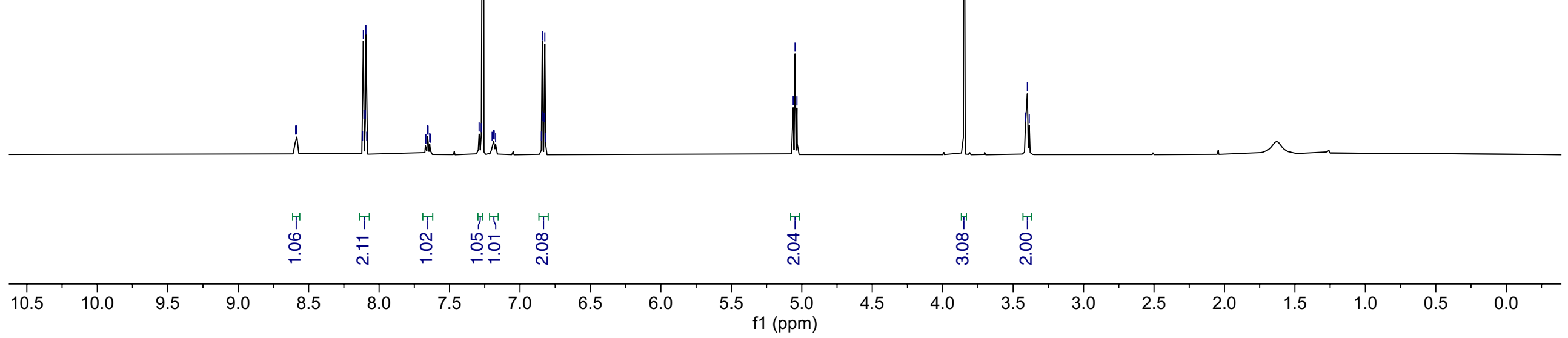

S52 
anoto.

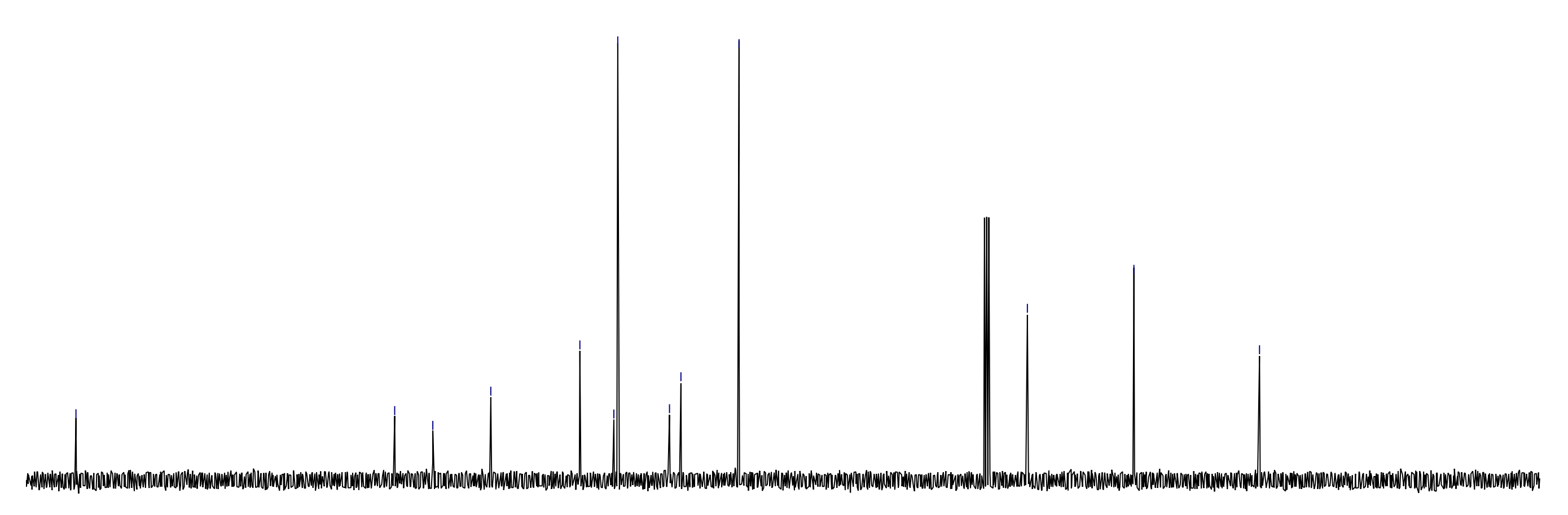


$500 \mathrm{MHz}, \mathrm{CDCl}_{3}$
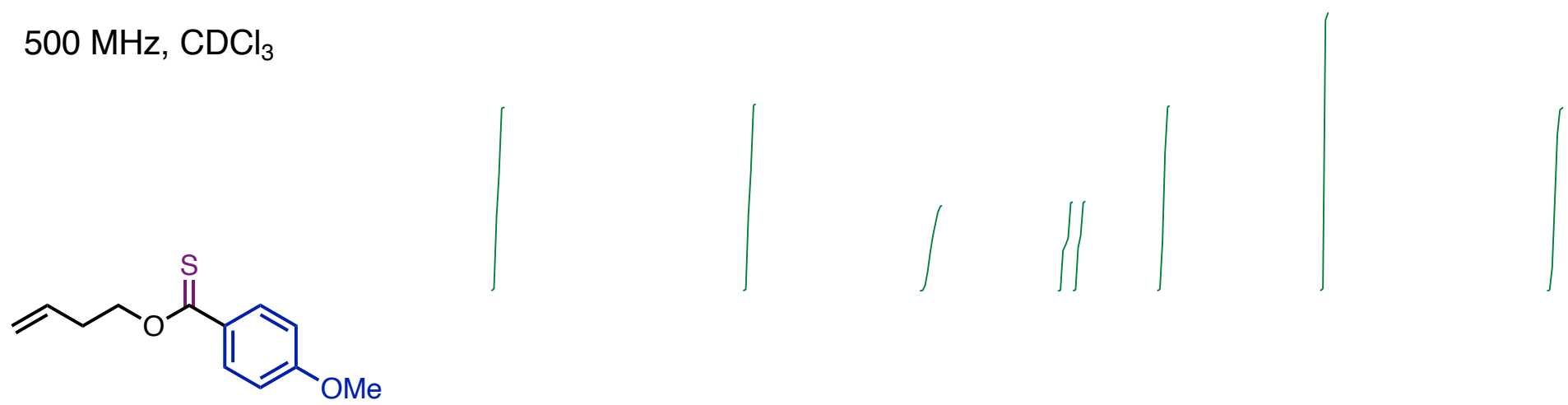

$3 h$

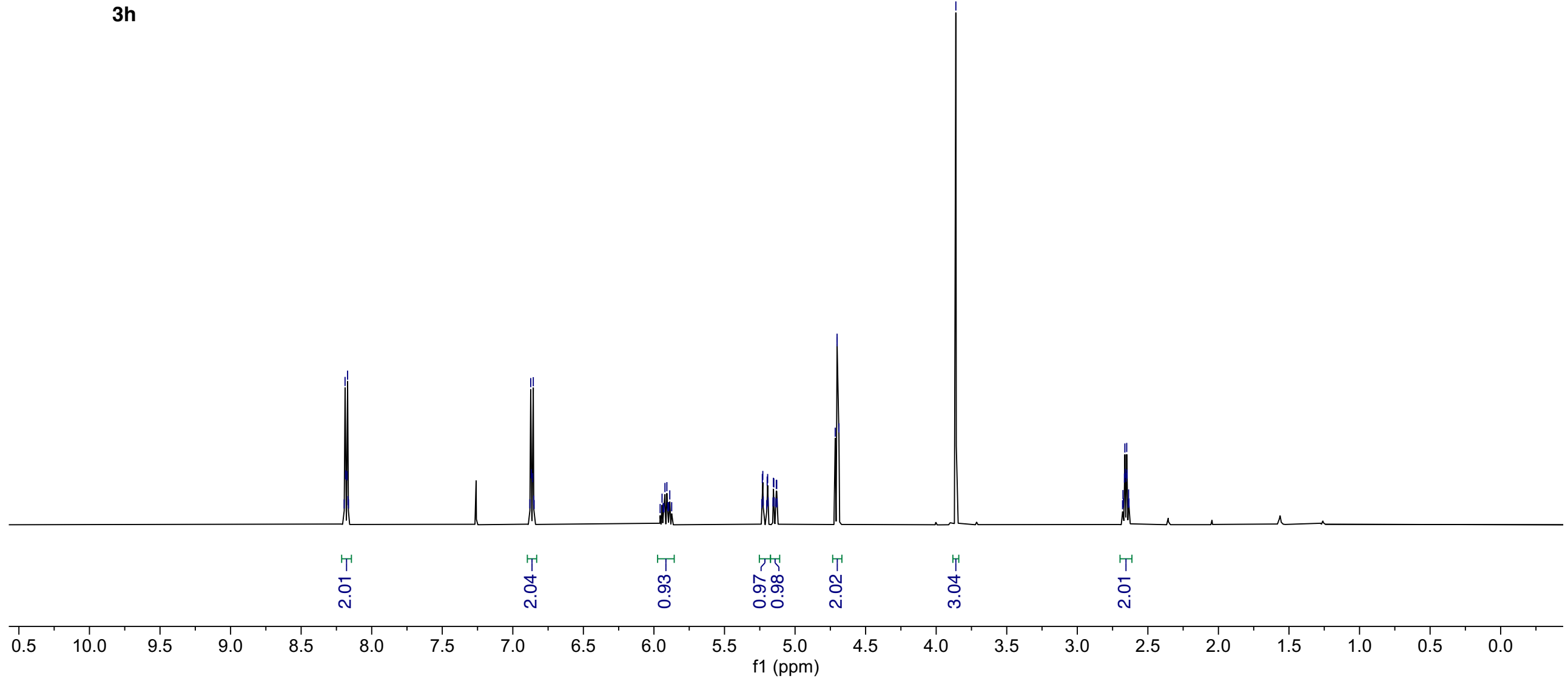


ํㅜㅇ

$\infty$
$\stackrel{\infty}{0}$
$\stackrel{0}{0}$

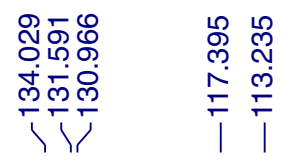

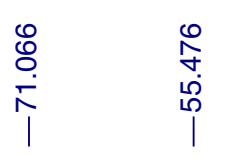

$\stackrel{\text { N }}{\text { กิ่ }}$<smiles>C=CCCOC(=S)c1ccc(OC)cc1</smiles>

3h

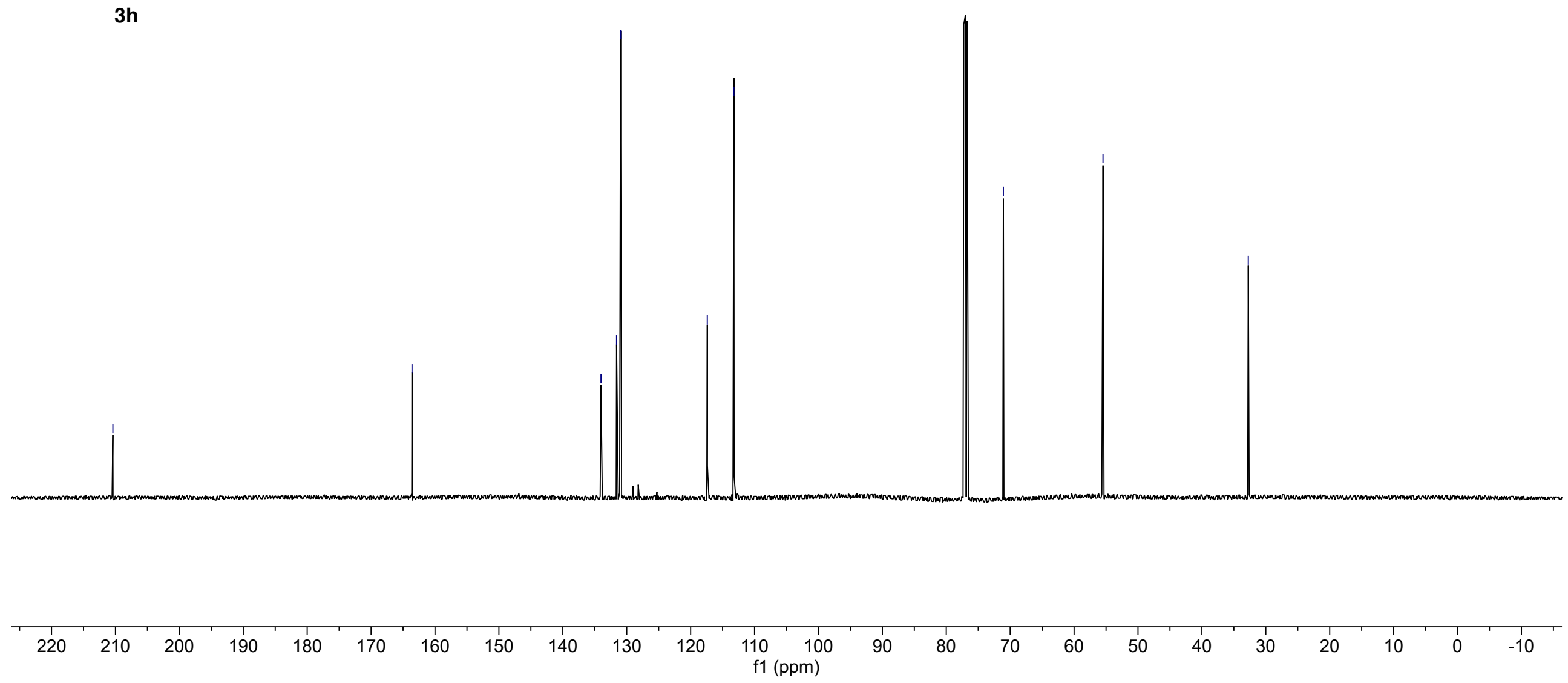

S55 
$500 \mathrm{MHz}, \mathrm{CDCl}_{3}$
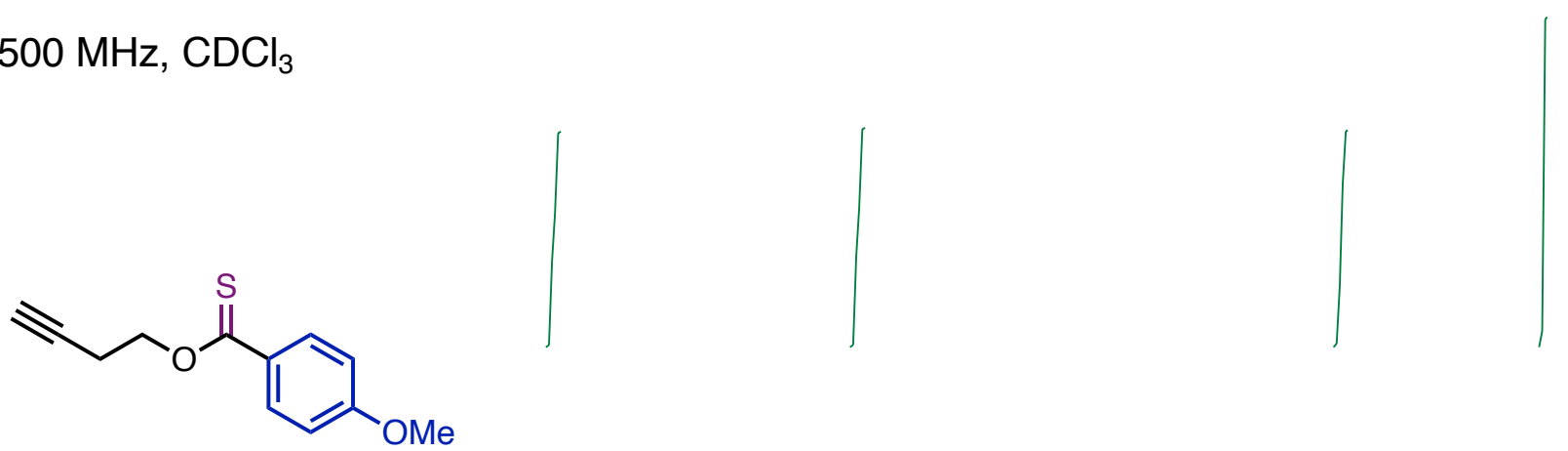

$3 \mathbf{i}$

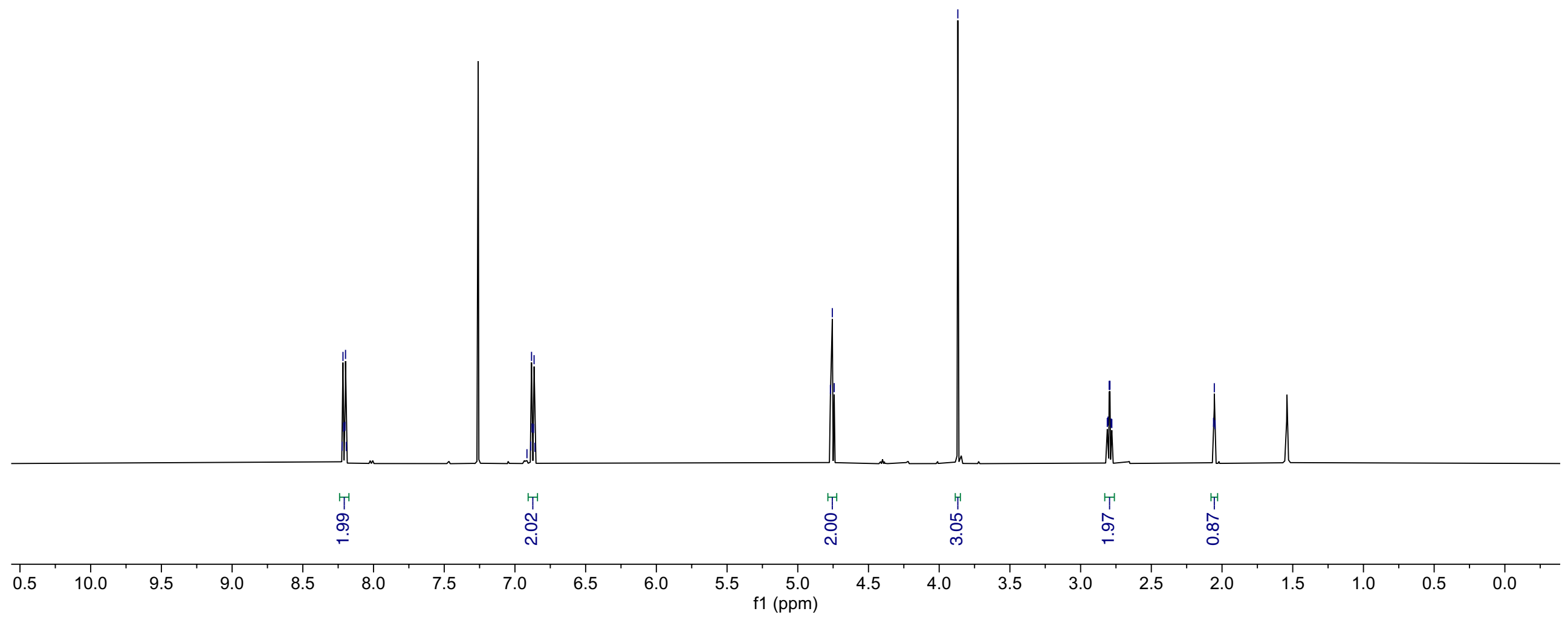


$\frac{8}{\stackrel{0}{0}}$

|

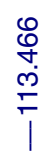

$\stackrel{\infty}{\infty}$

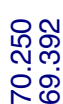

مُ

0
$\vdots$
$\infty$
$\infty$
$\infty$

$126 \mathrm{MHz}^{\mathrm{CDCl}_{3}}$

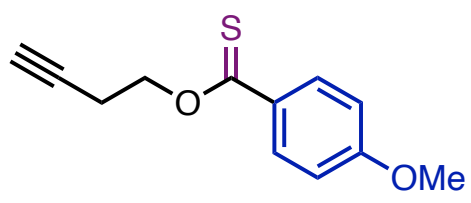

$3 \mathbf{i}$

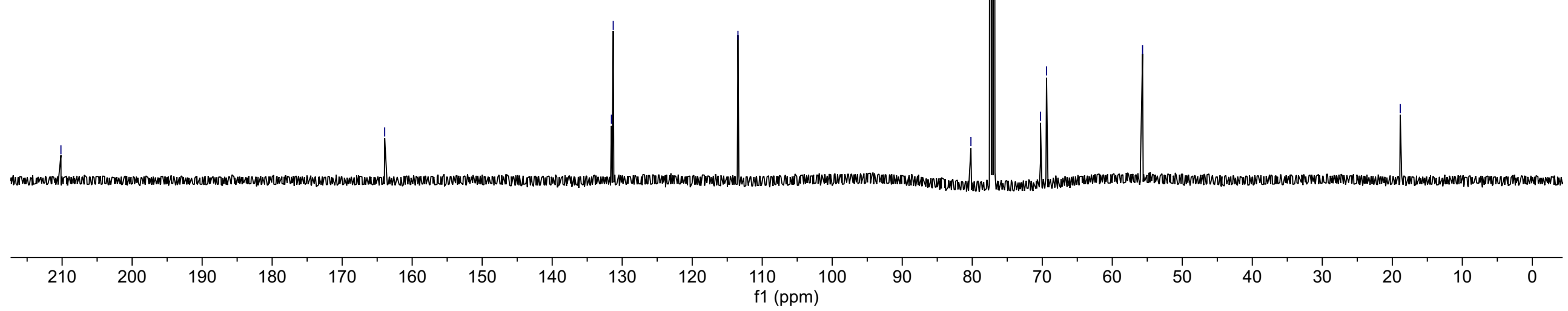

S57 


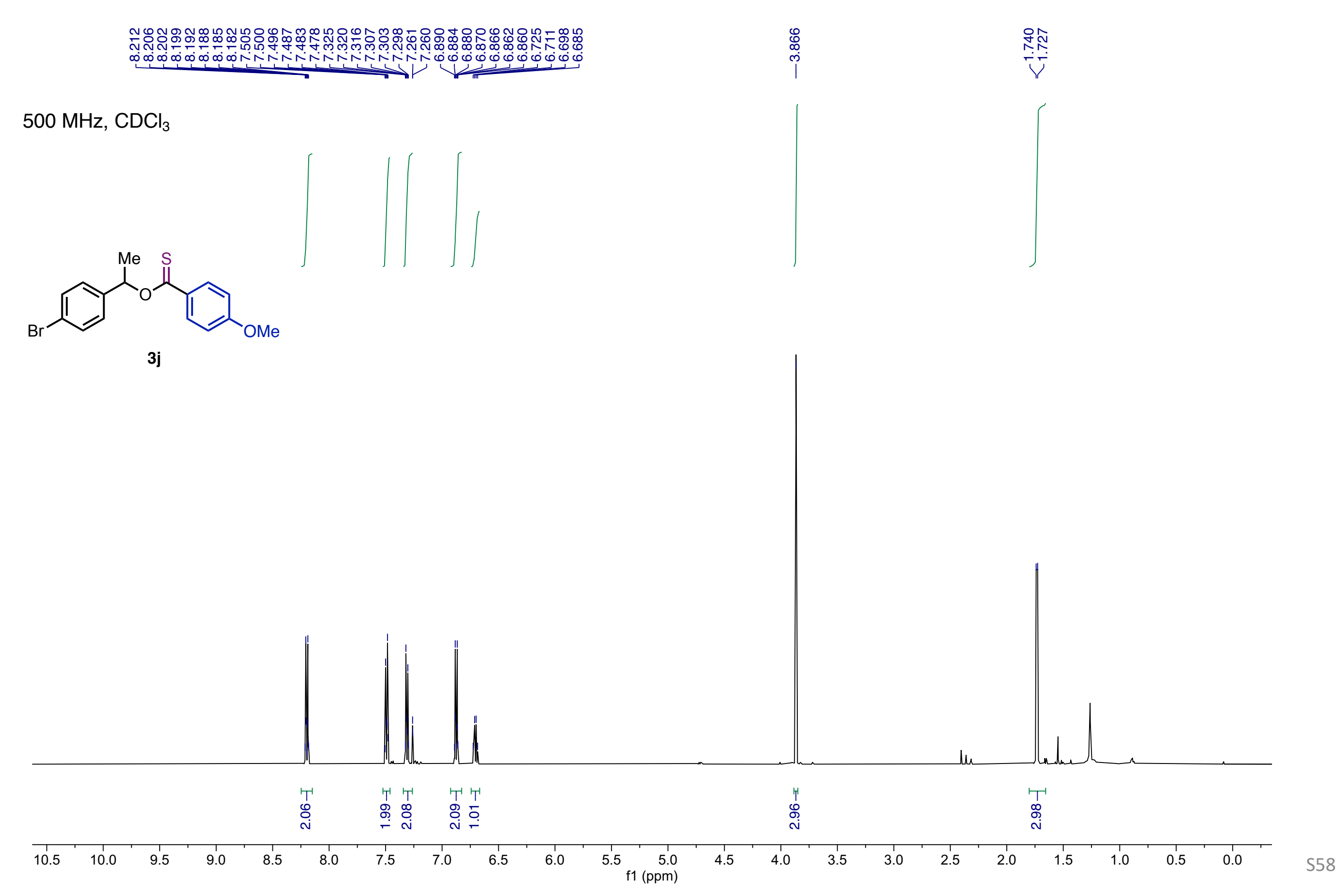


$\begin{array}{llll}1 & 1\end{array}$

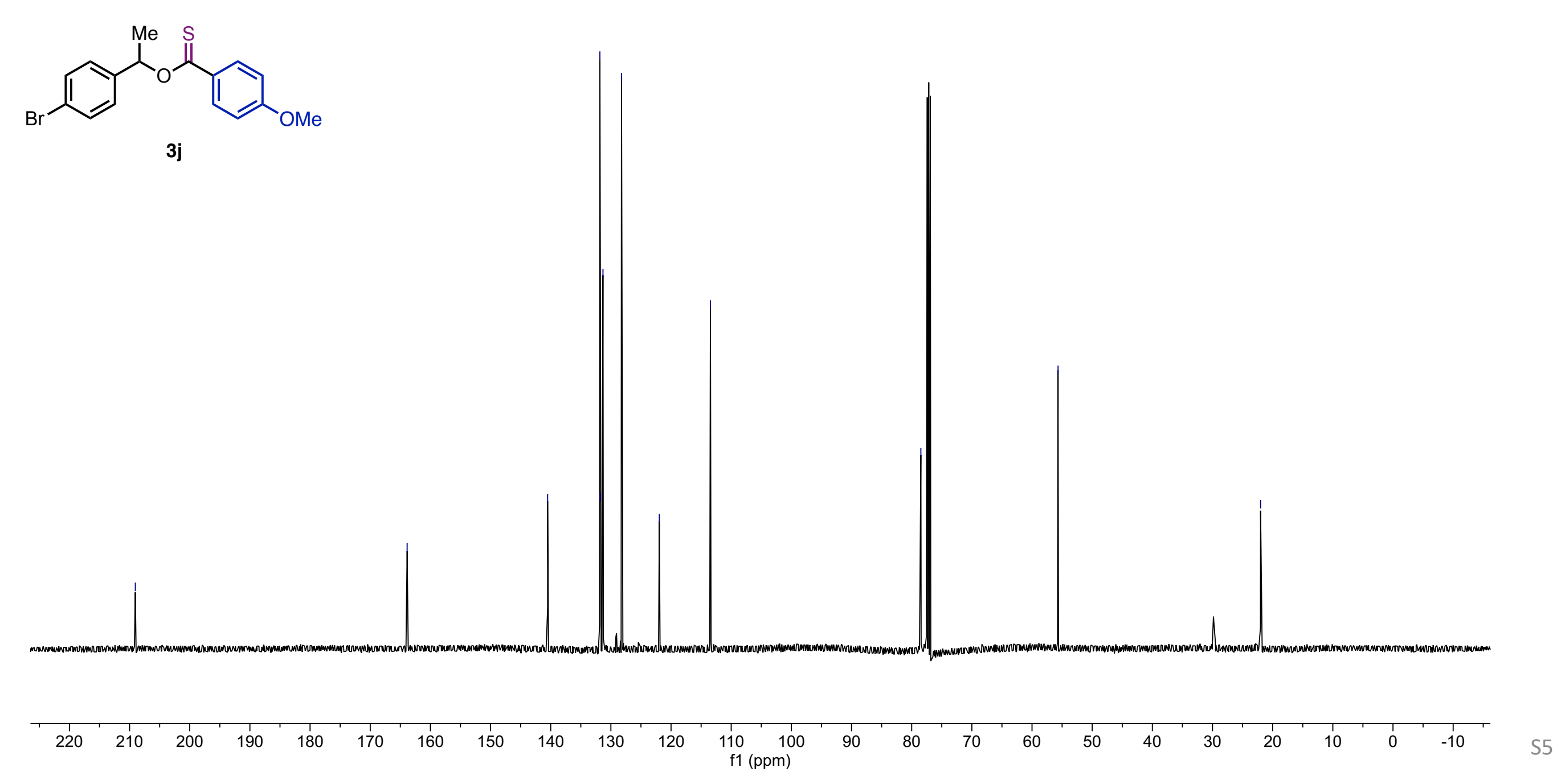


$400 \mathrm{MHz}, \mathrm{CDCl}_{3}$
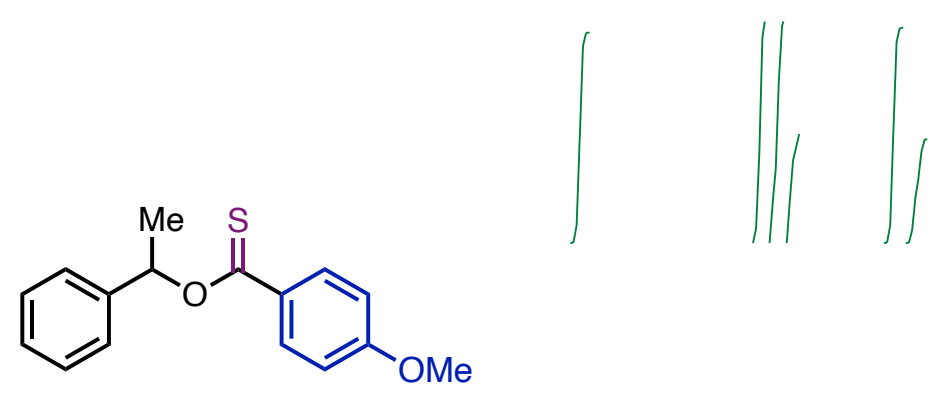

3k
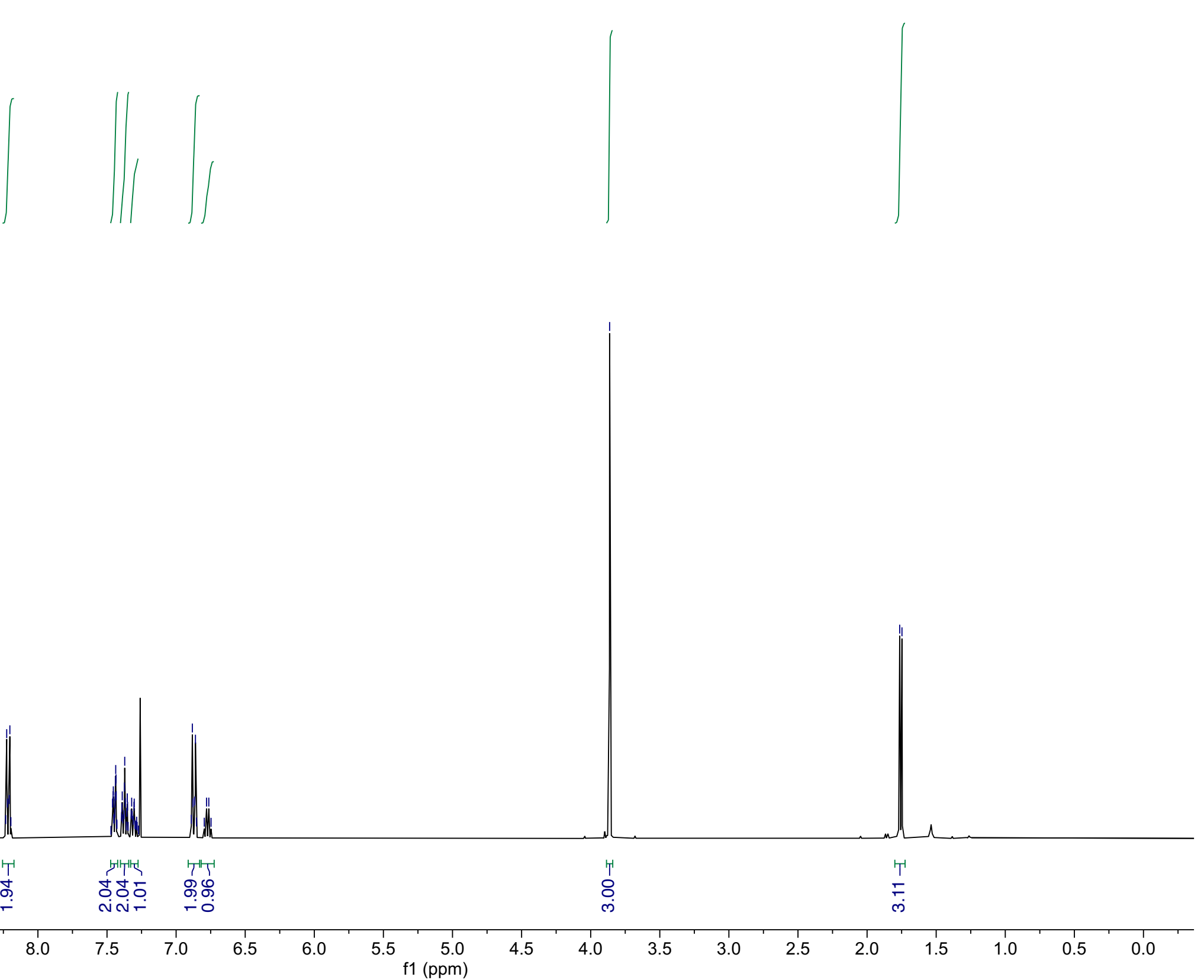
$\begin{array}{llll}1 & 1\end{array}$

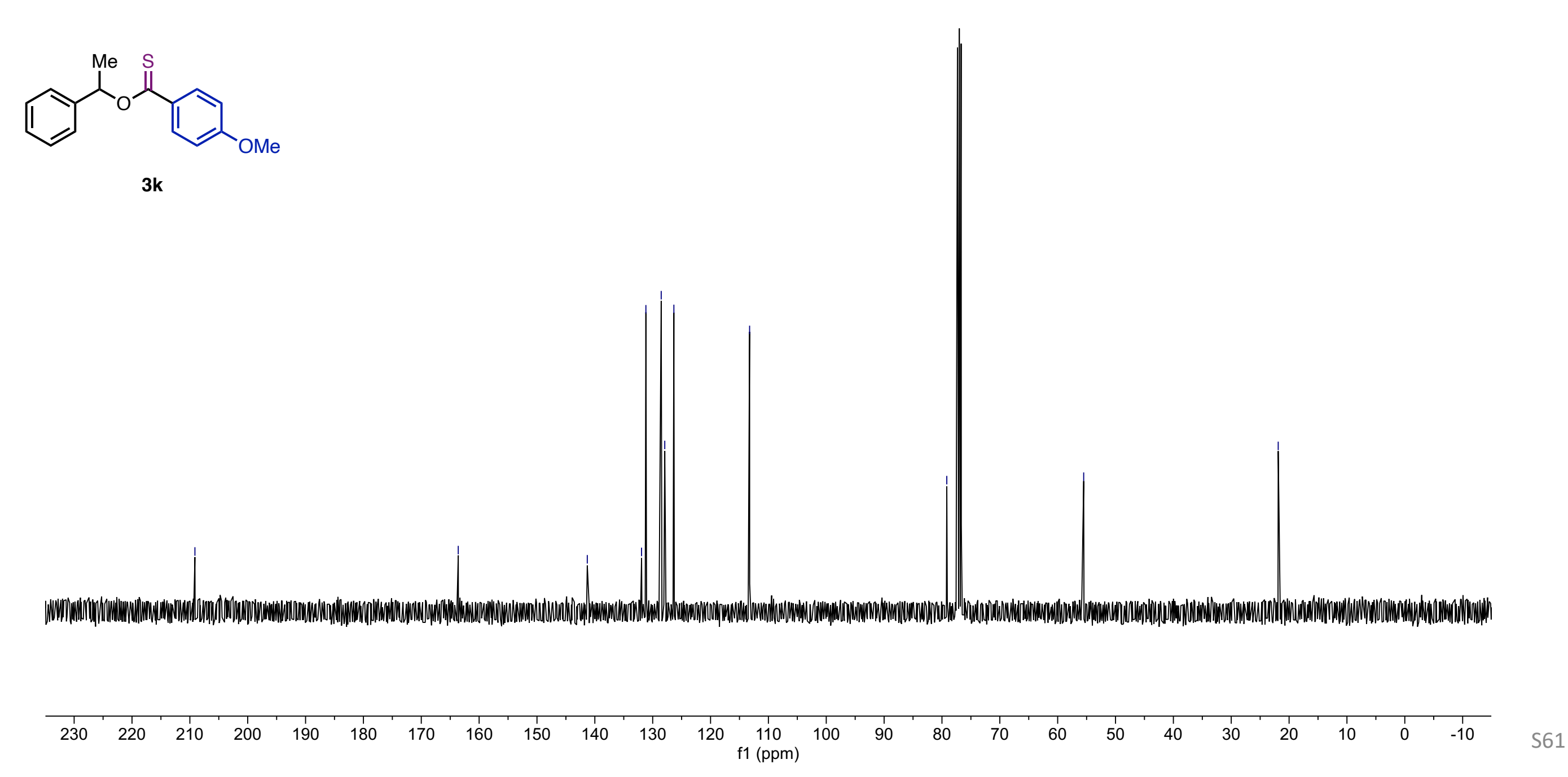


$500 \mathrm{MHz}, \mathrm{CDCl}_{3}$
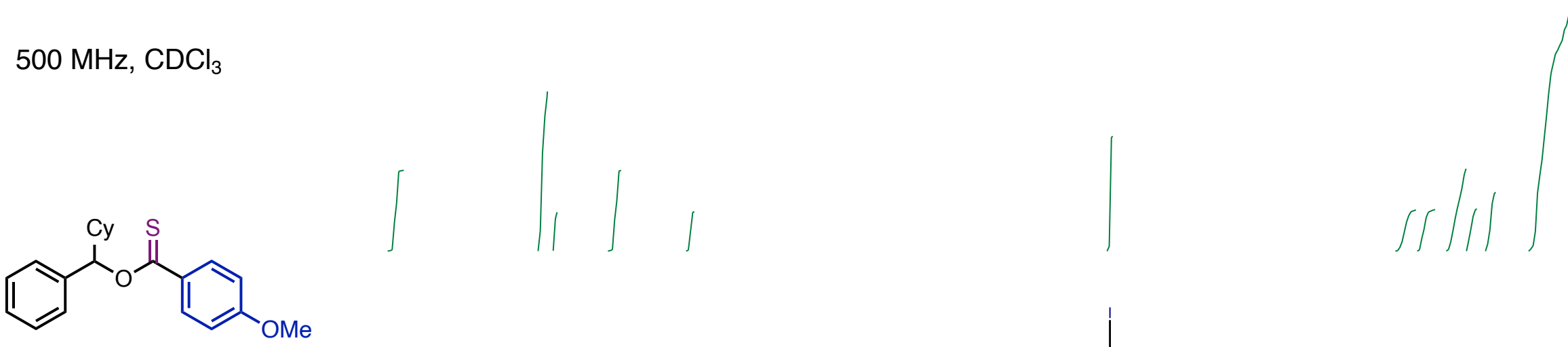

31

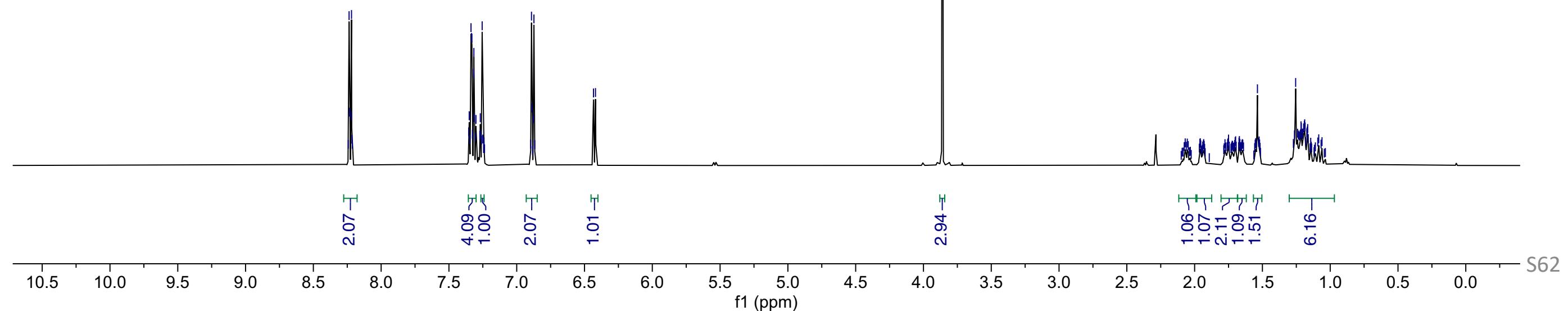


$126 \mathrm{MHz}, \mathrm{CDCl}_{3}$

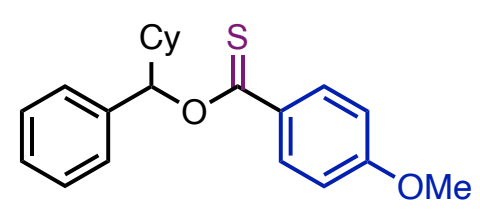

31

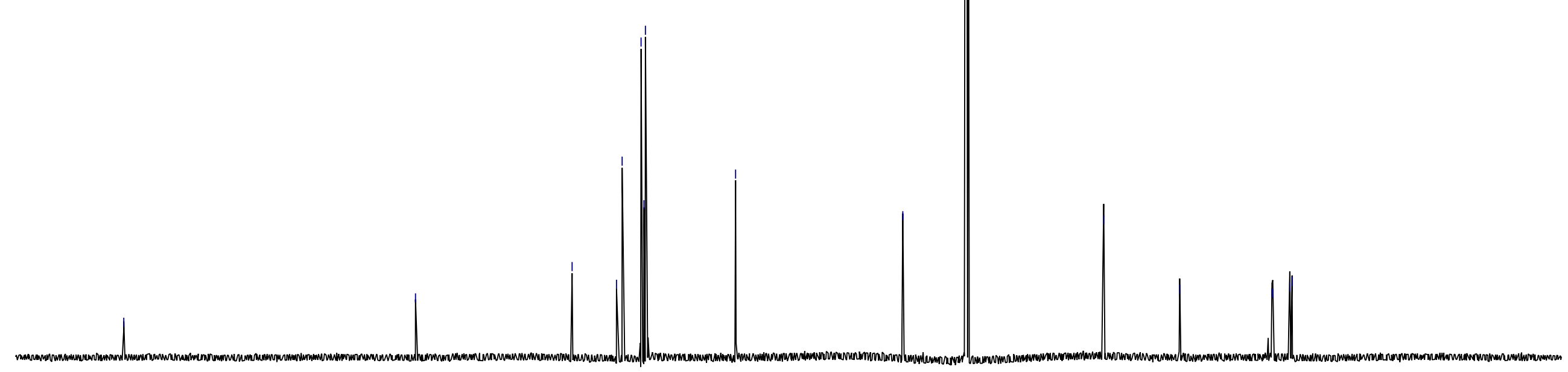

220

$210 \quad 200$ 


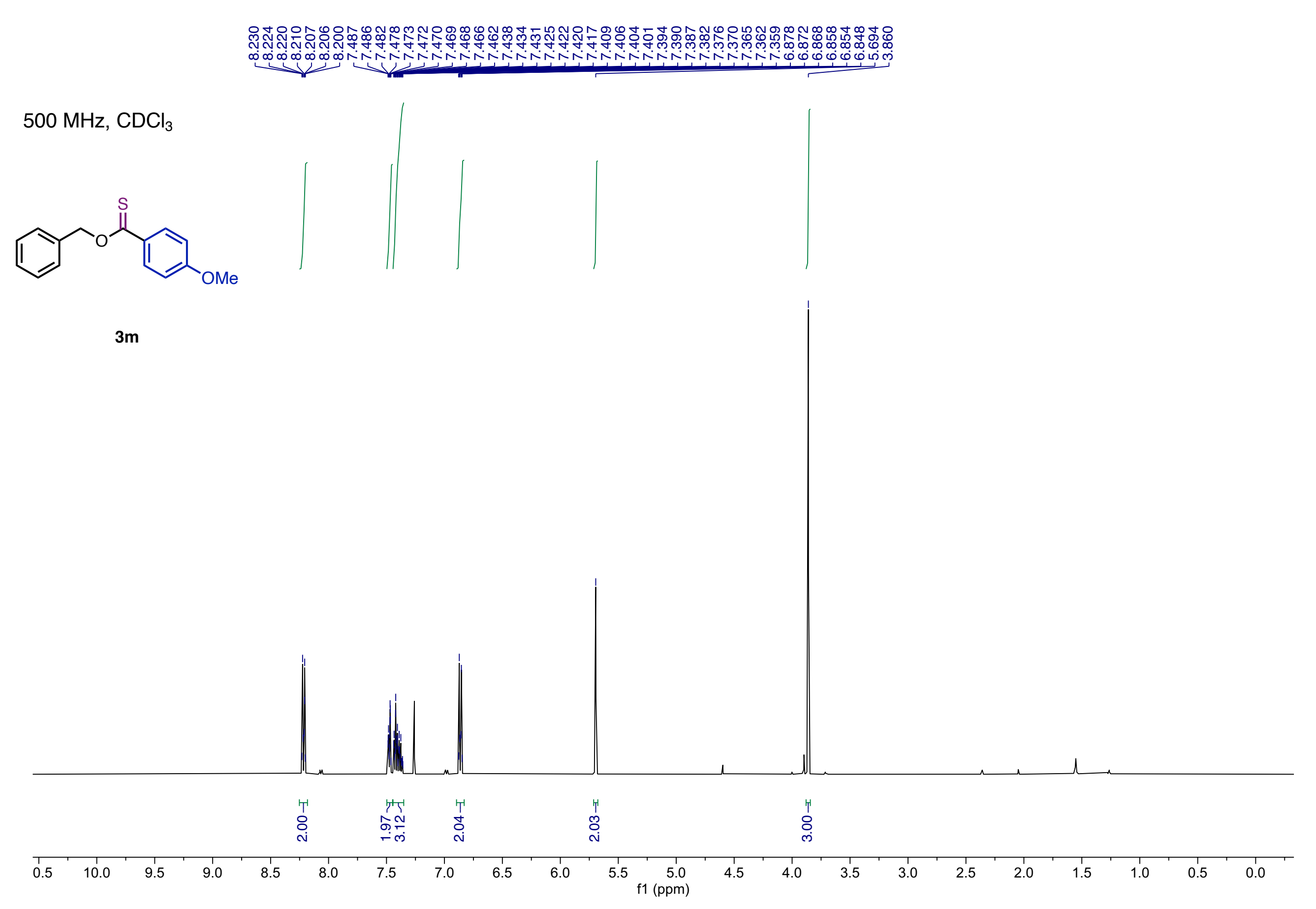




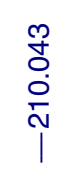

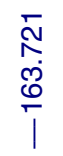

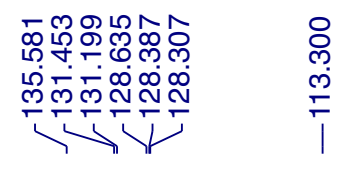

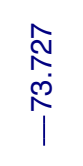

0
$\substack{0 \\ 0 \\ 0}$
1<smiles>COc1ccc(C(=S)OCc2ccccc2)cc1</smiles>

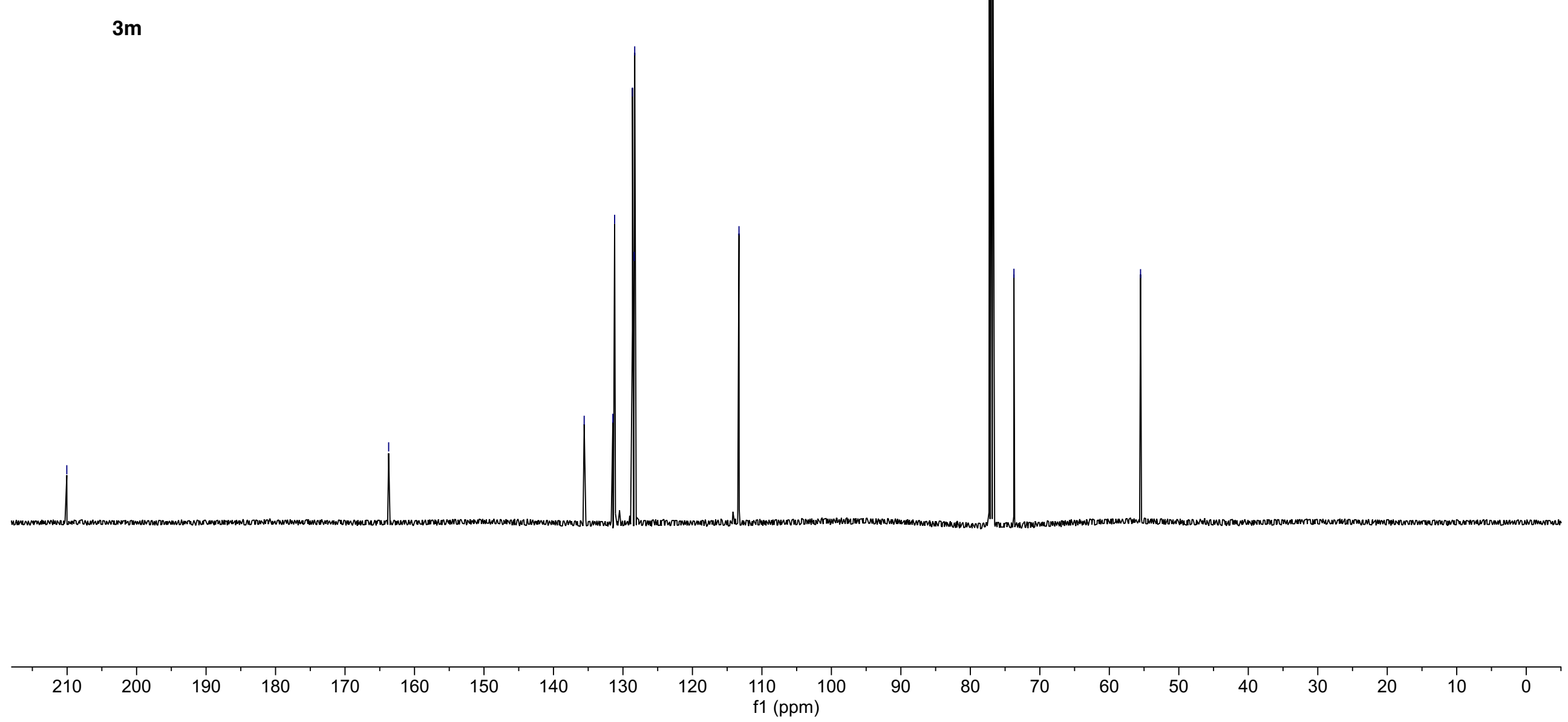

S65 


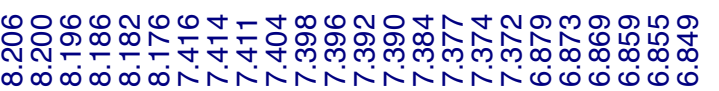

în

$\mathscr{0}$
$\infty$
$\infty$
$\infty$<smiles>COc1ccc(C(=S)OCc2ccc(Cl)cc2)cc1</smiles>

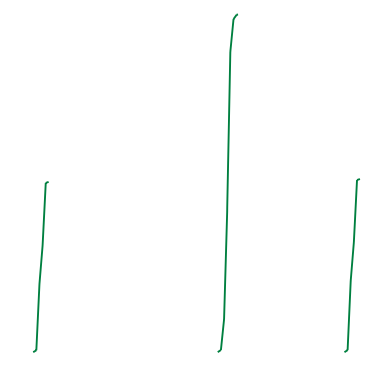

3n

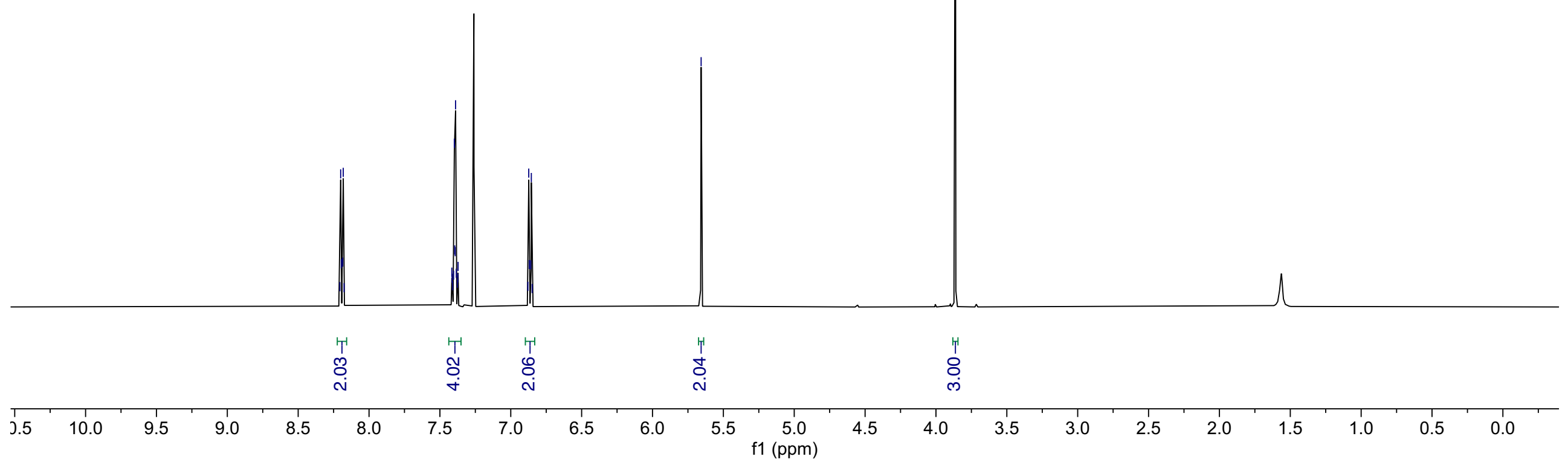

S66 


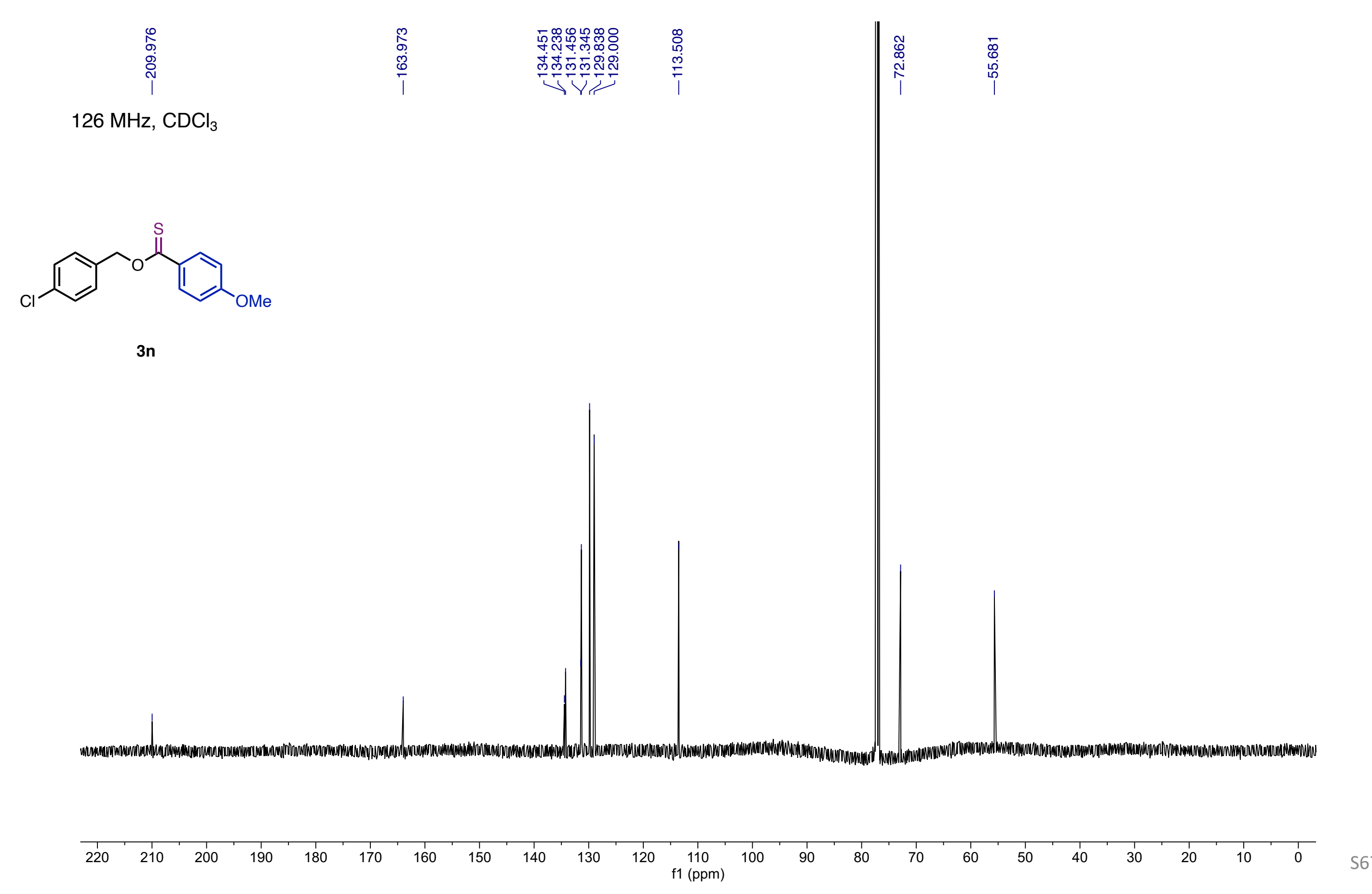


$500 \mathrm{MHz}, \mathrm{CDCl}_{3}$
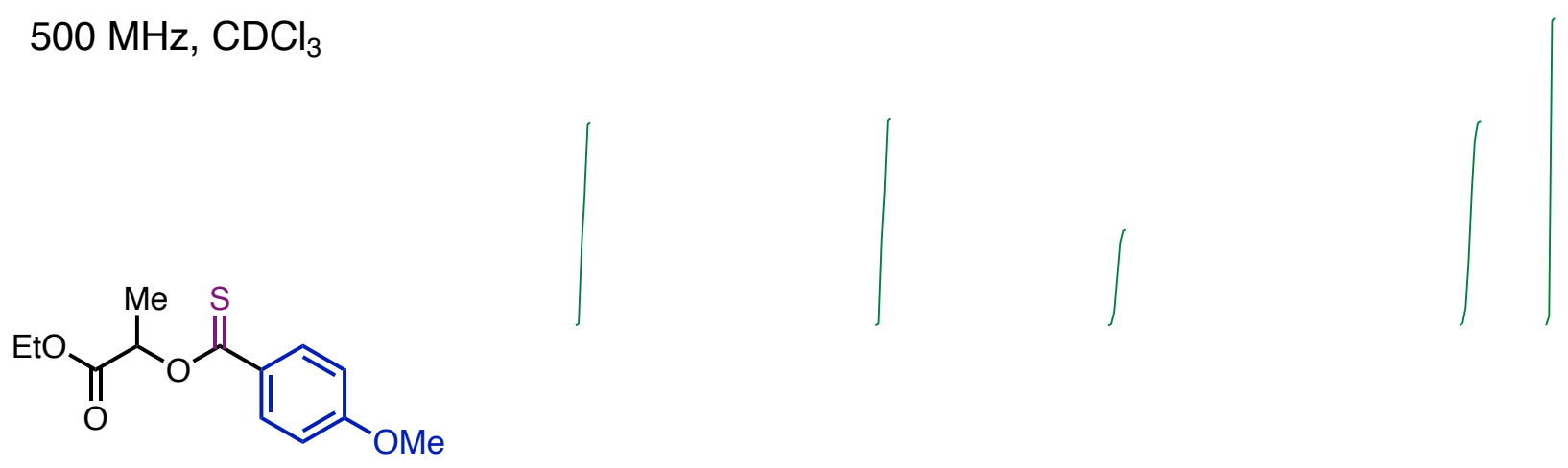

30
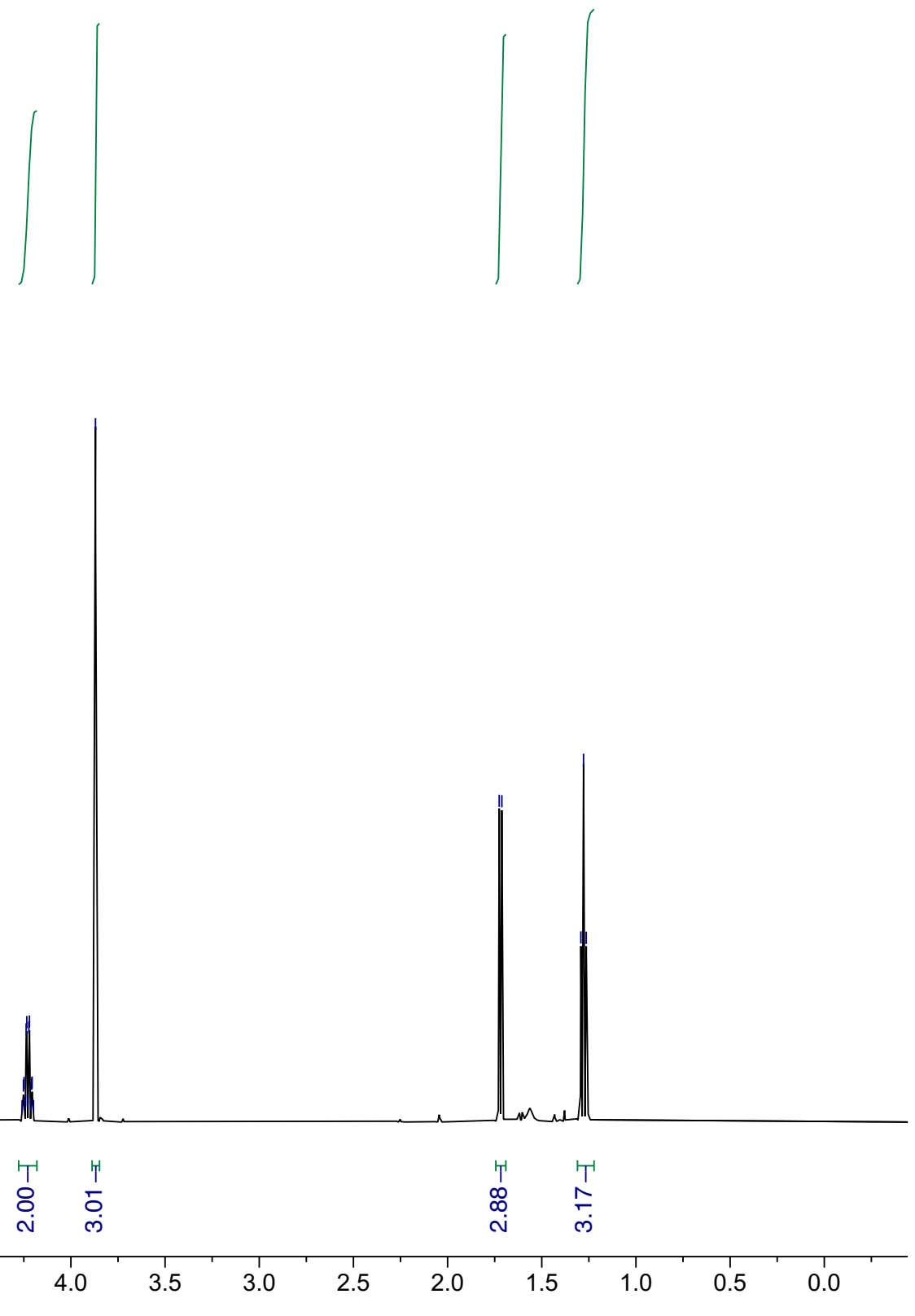
$126 \mathrm{MHz}, \mathrm{CDCl}_{3}$

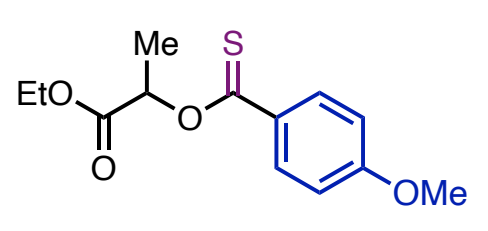

30

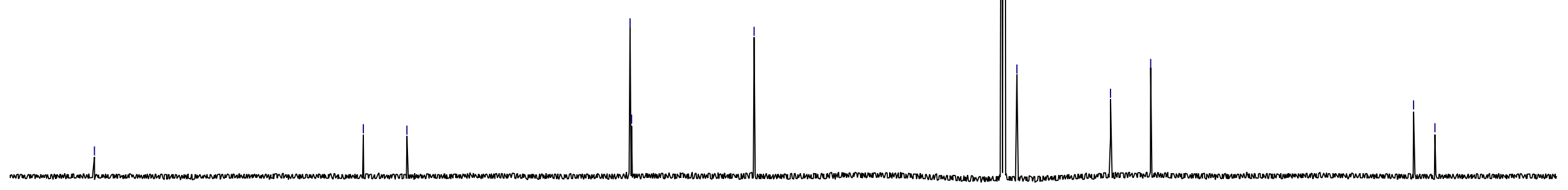




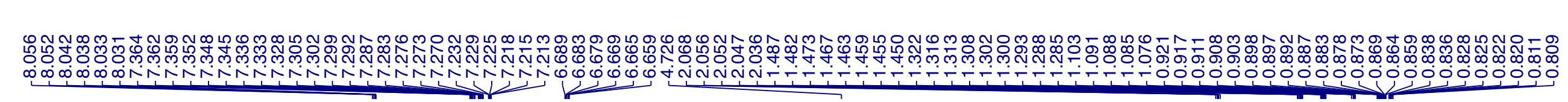
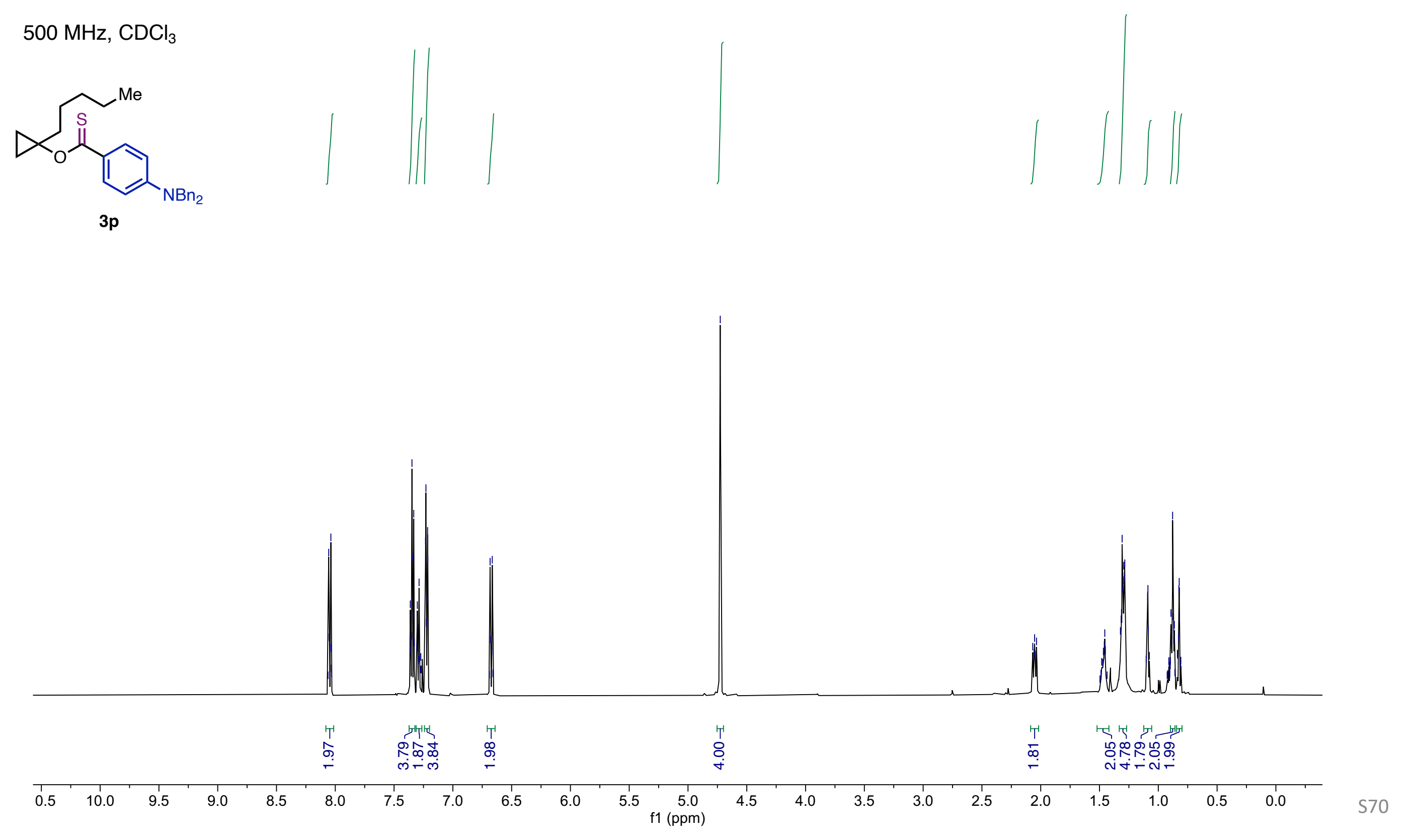
$126 \mathrm{MHz}, \mathrm{CDCl}_{3}$

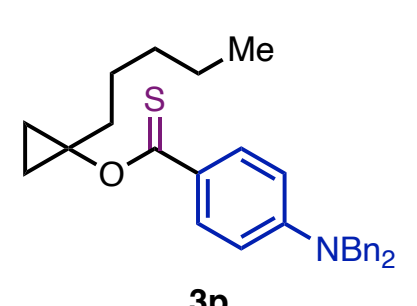

$3 p$

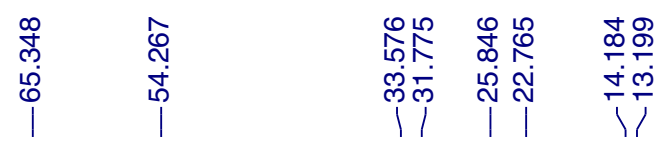

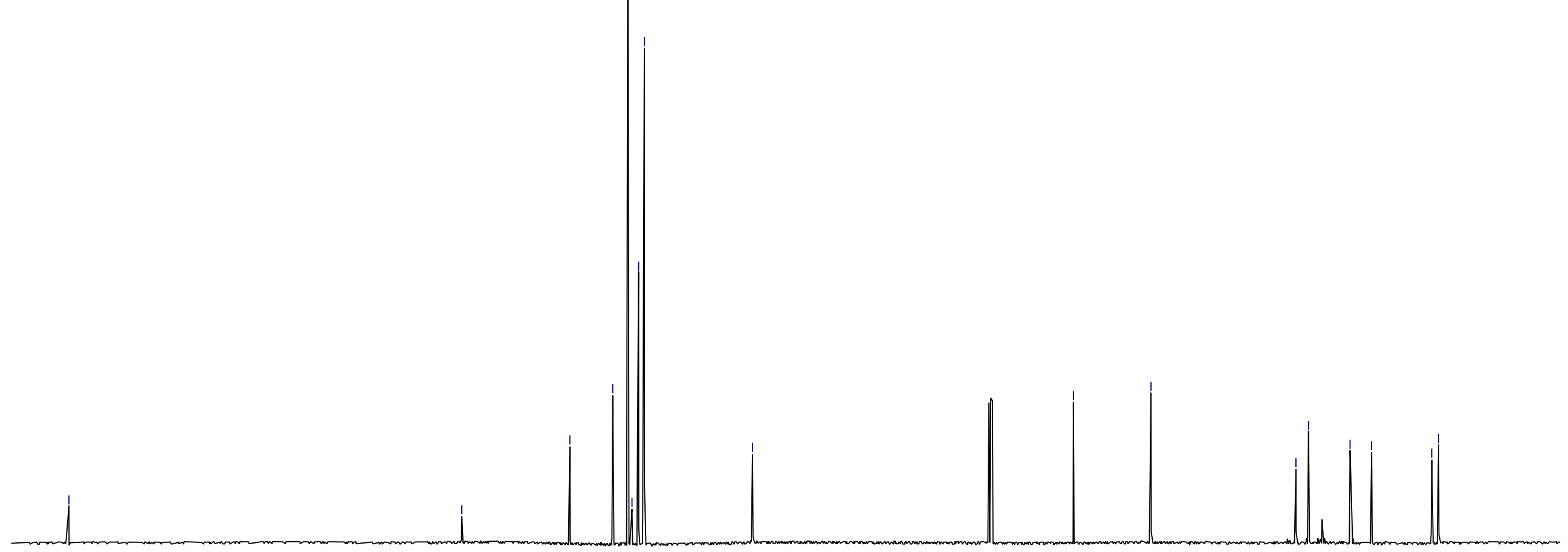


$500 \mathrm{MHz}, \mathrm{CDCl}_{3}$<smiles>CCCCCC1(OC(=S)c2ccc3ccccc3c2)CC1</smiles><smiles>C#CC=CC#C</smiles>

$3 q$
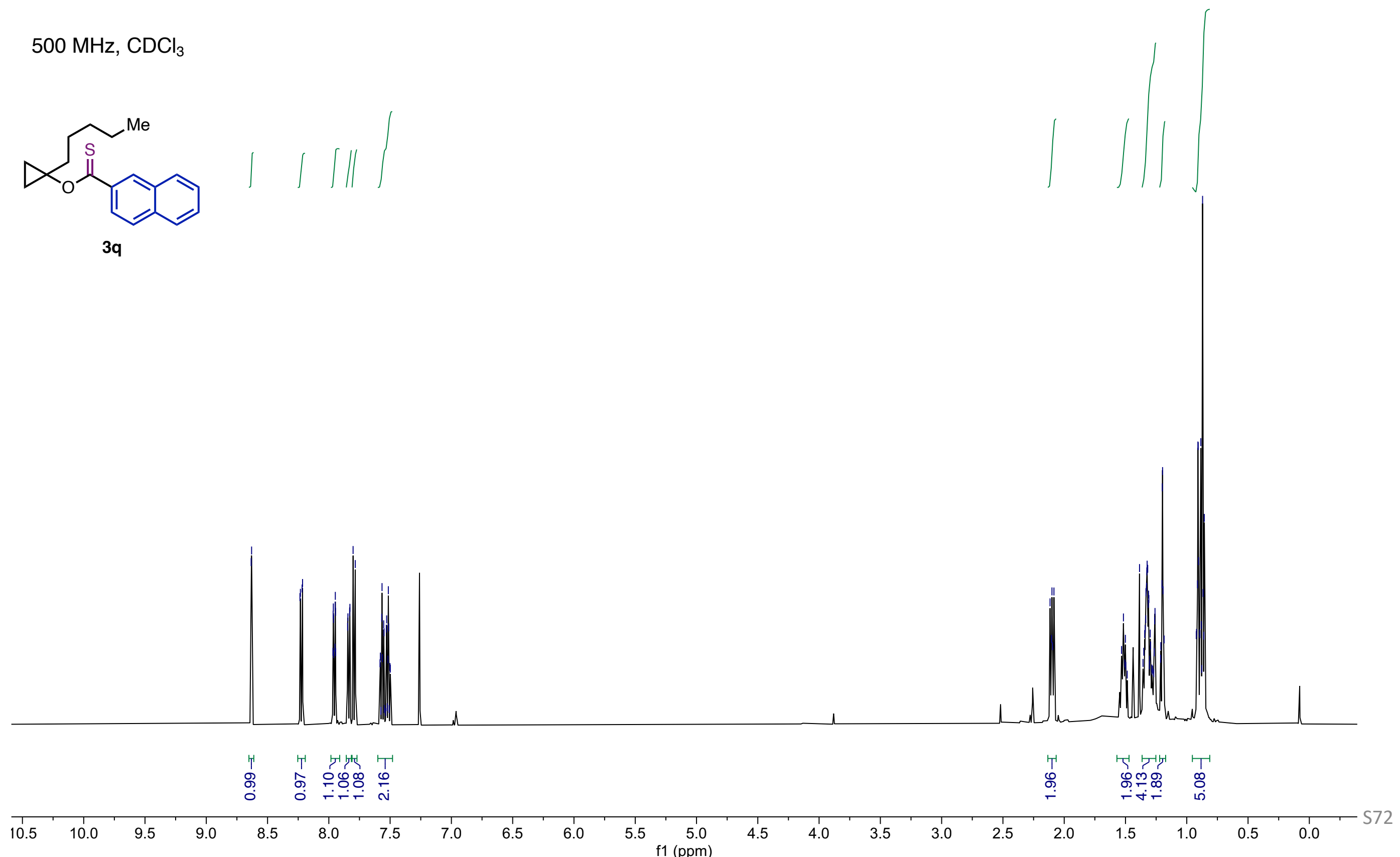


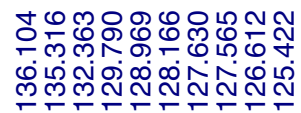

10
$\stackrel{1}{m}$
0
0

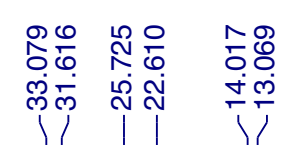

$126 \mathrm{MHz}, \mathrm{CDCl}_{3}$

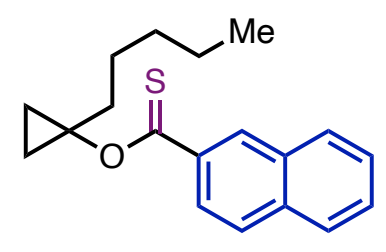

$3 q$
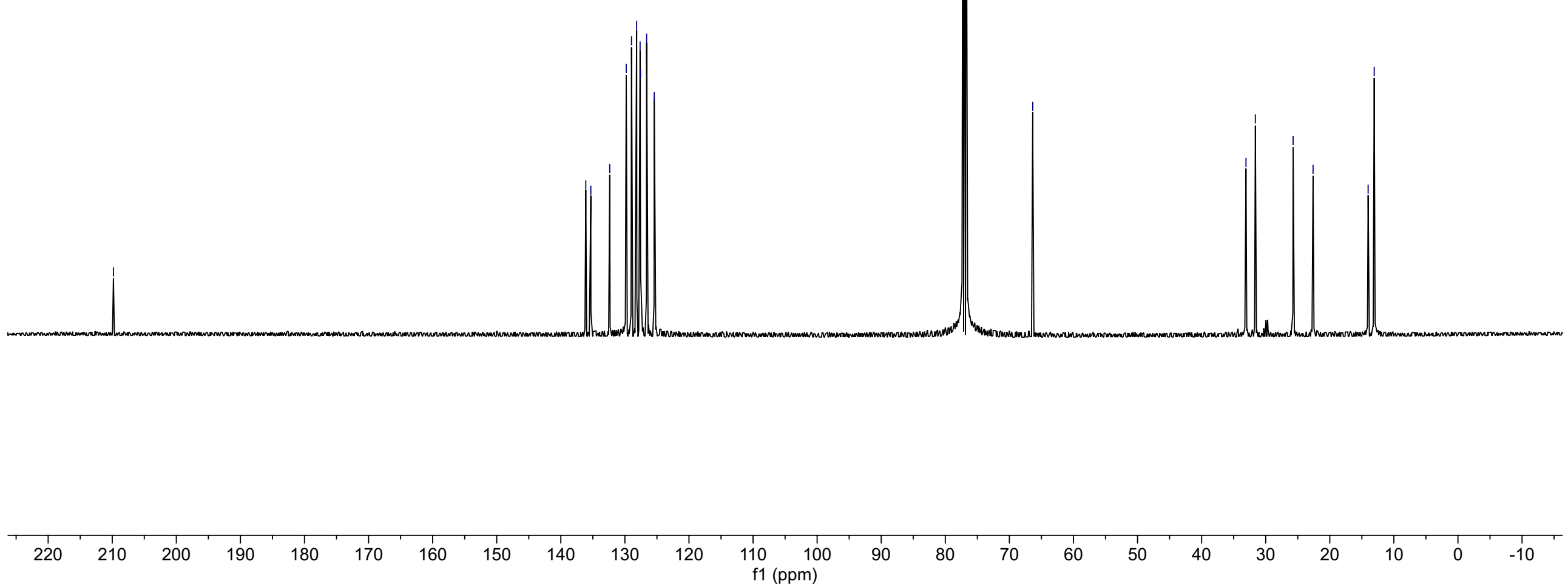

S73 
$400 \mathrm{MHz}, \mathrm{CDCl}_{3}$<smiles>O=C(OCc1ccccc1)c1ccsc1</smiles>

$3 r$
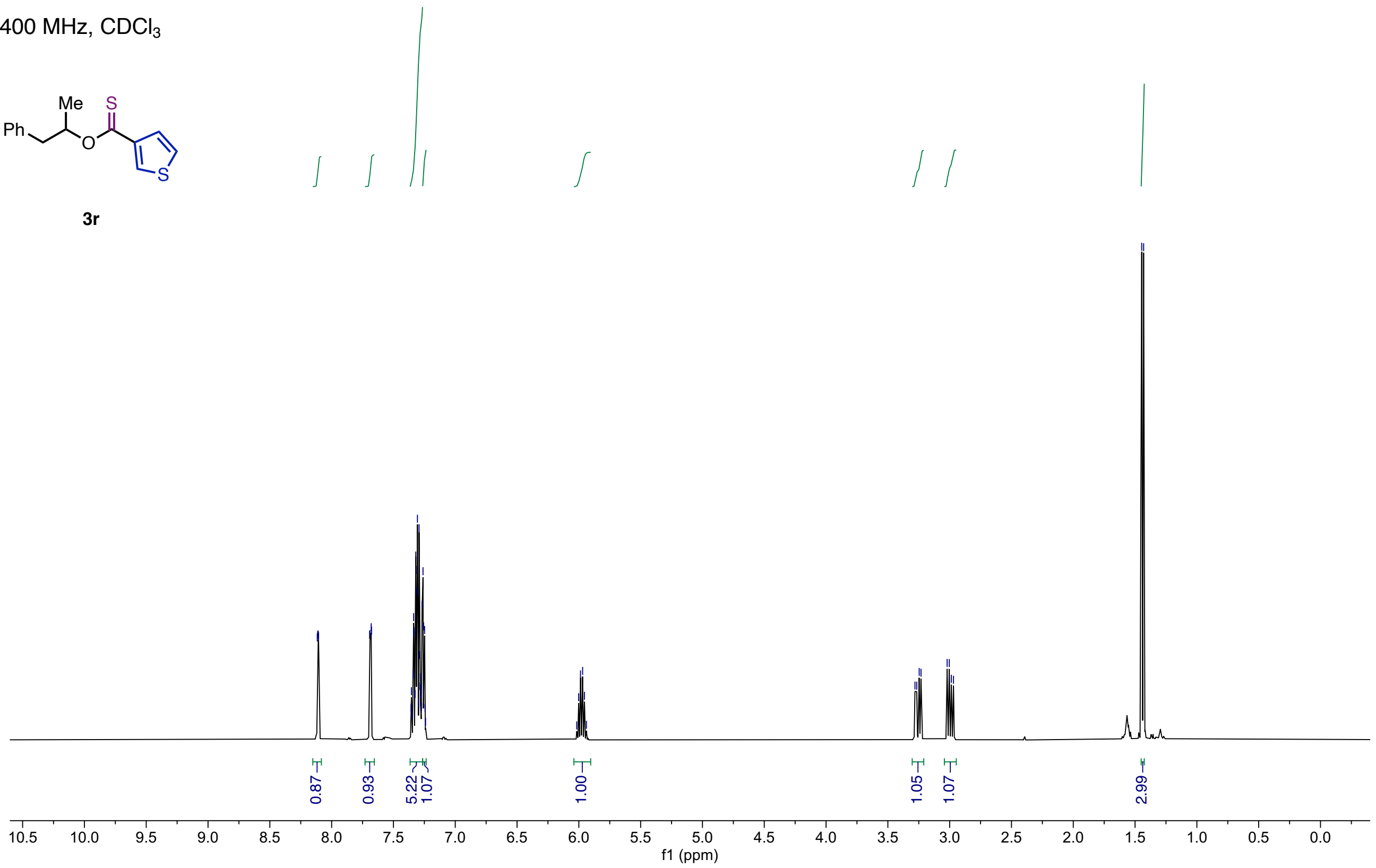
$101 \mathrm{MHz}, \mathrm{CDCl}_{3}$

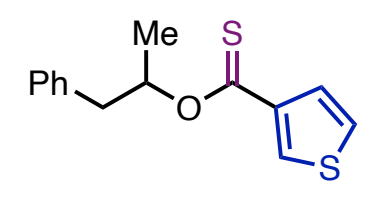

$101 \mathrm{MHz}, \mathrm{CDCl}_{3}$

$3 r$

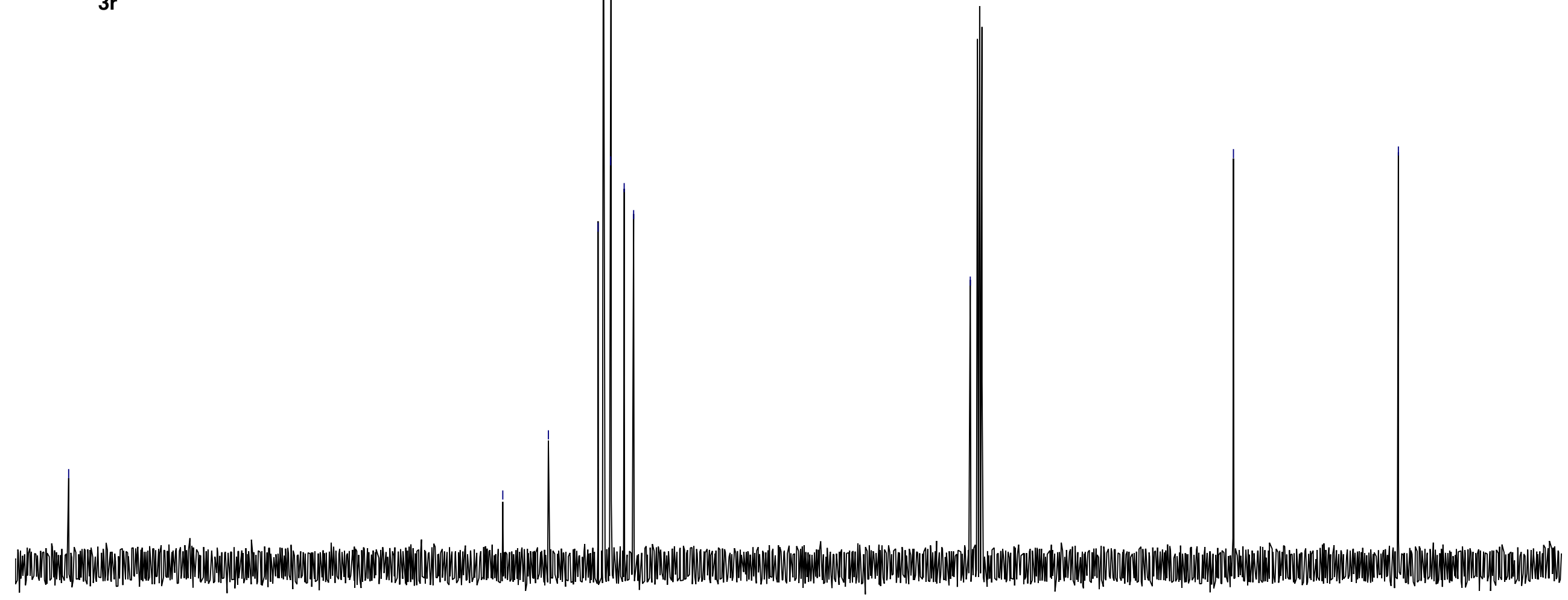




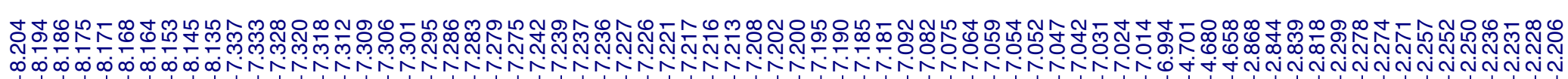

$400 \mathrm{MHz}, \mathrm{CDCl}_{3}$<smiles>Fc1ccc(C(=S)OCCCc2ccccc2)cc1</smiles>
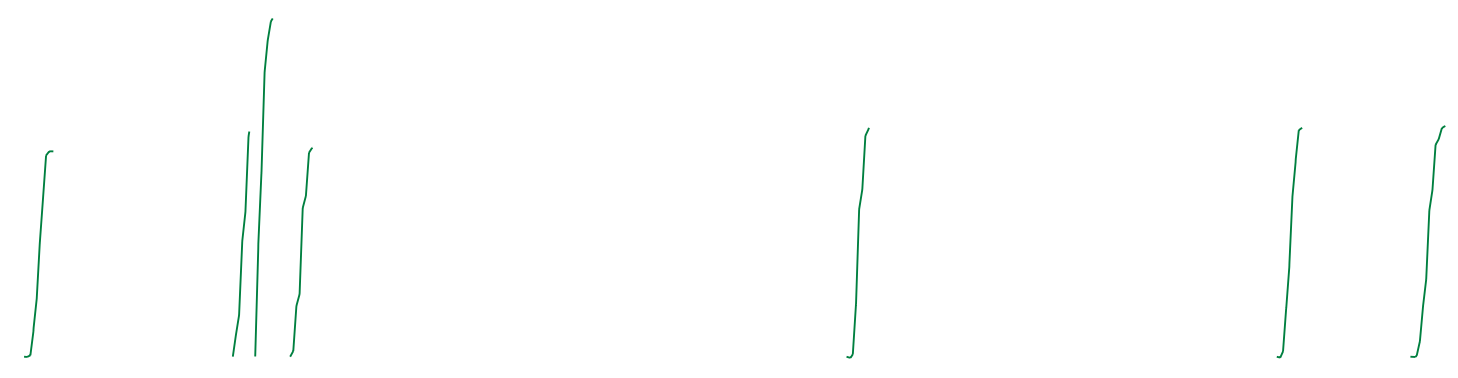

$3 s$

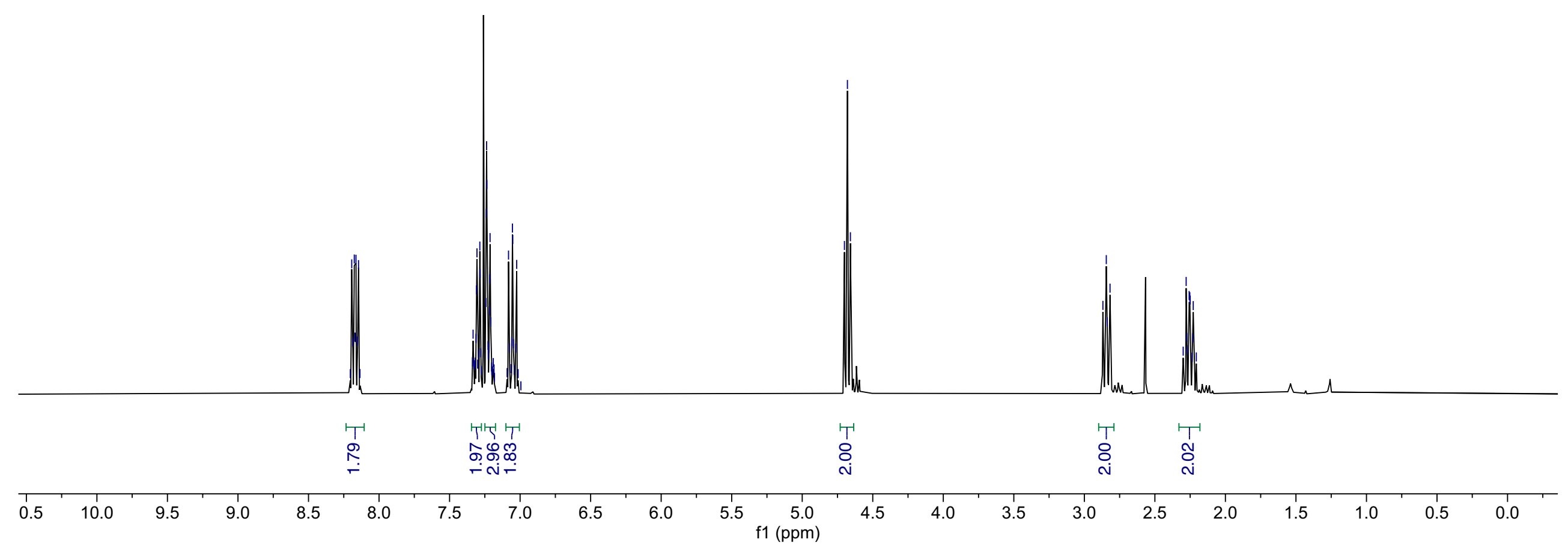

576 


$$
1
$$


$\cot 2$.

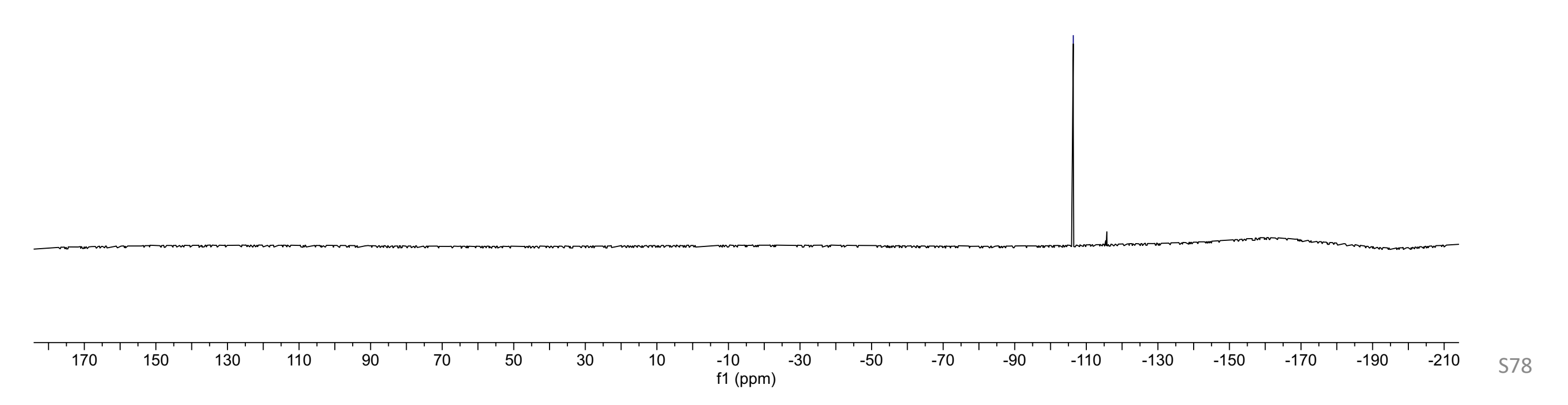


$400 \mathrm{MHz}, \mathrm{CDCl}_{3}$<smiles>COc1ccc(C(=S)OCc2ccccc2)cc1OC</smiles><smiles>C1CCCC1</smiles>

3t
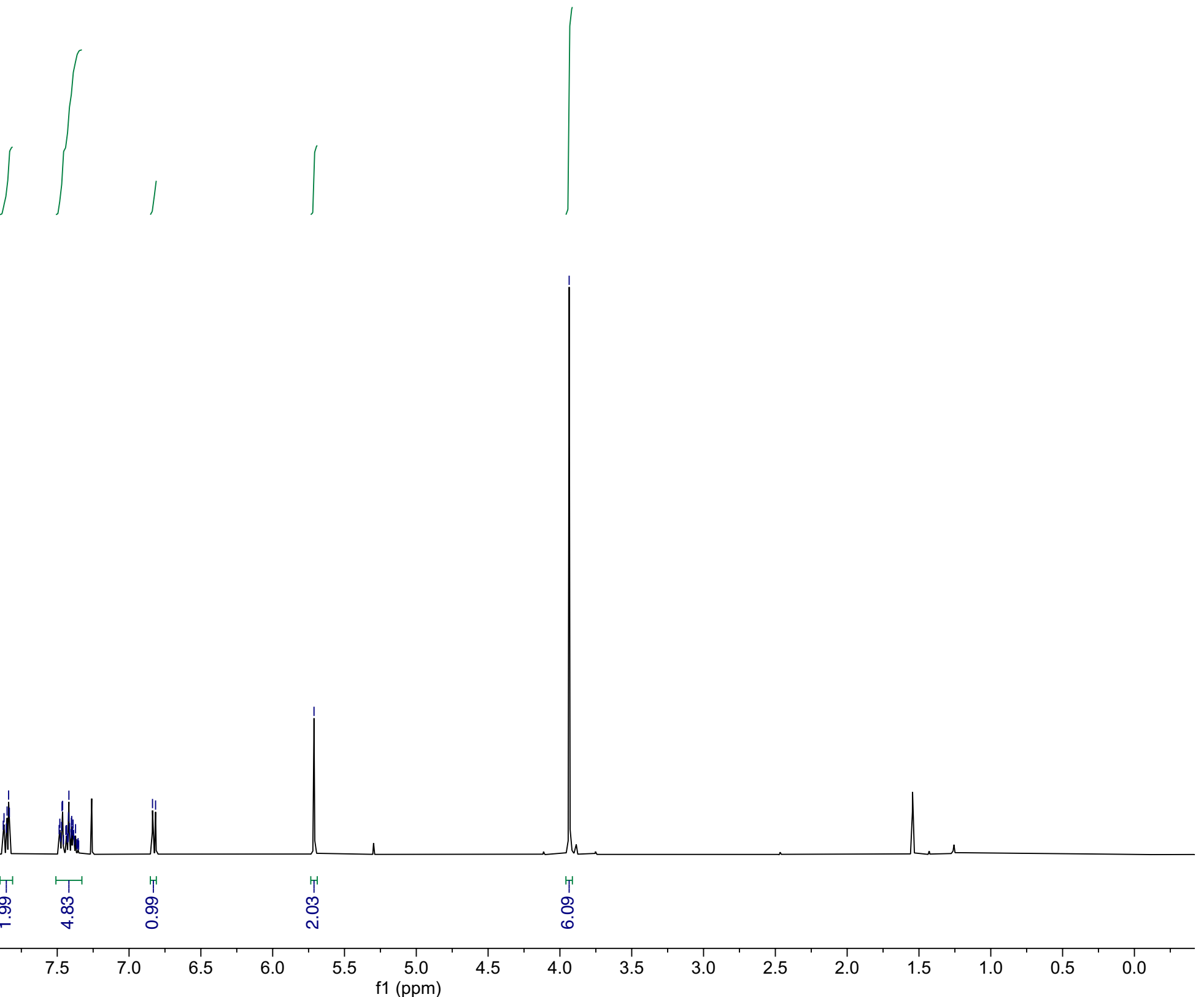


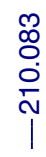

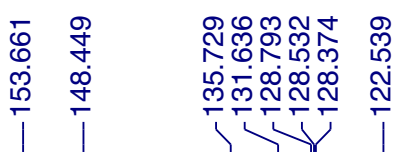

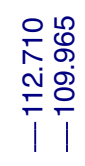

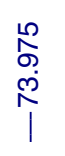

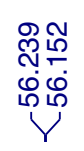

$101 \mathrm{MHz}, \mathrm{CDCl}_{3}$<smiles>COc1ccc(C(=S)OCc2ccccc2)cc1OC</smiles>

$3 t$
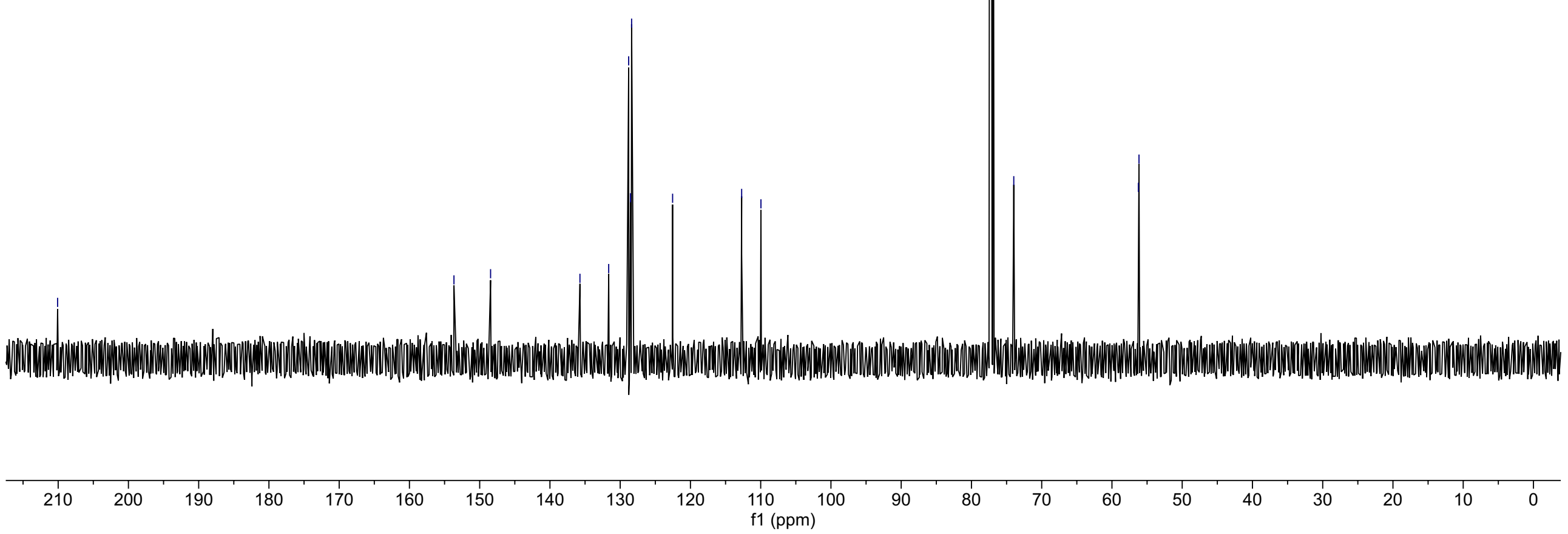

$\mathrm{S} 80$ 
$500 \mathrm{MHz}, \mathrm{CDCl}_{3}$<smiles>CC(Cc1ccccc1)OC(=S)c1ccc2[nH]ccc2c1</smiles>

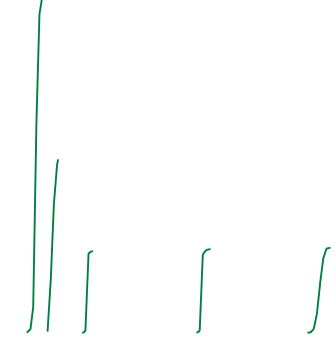

3u
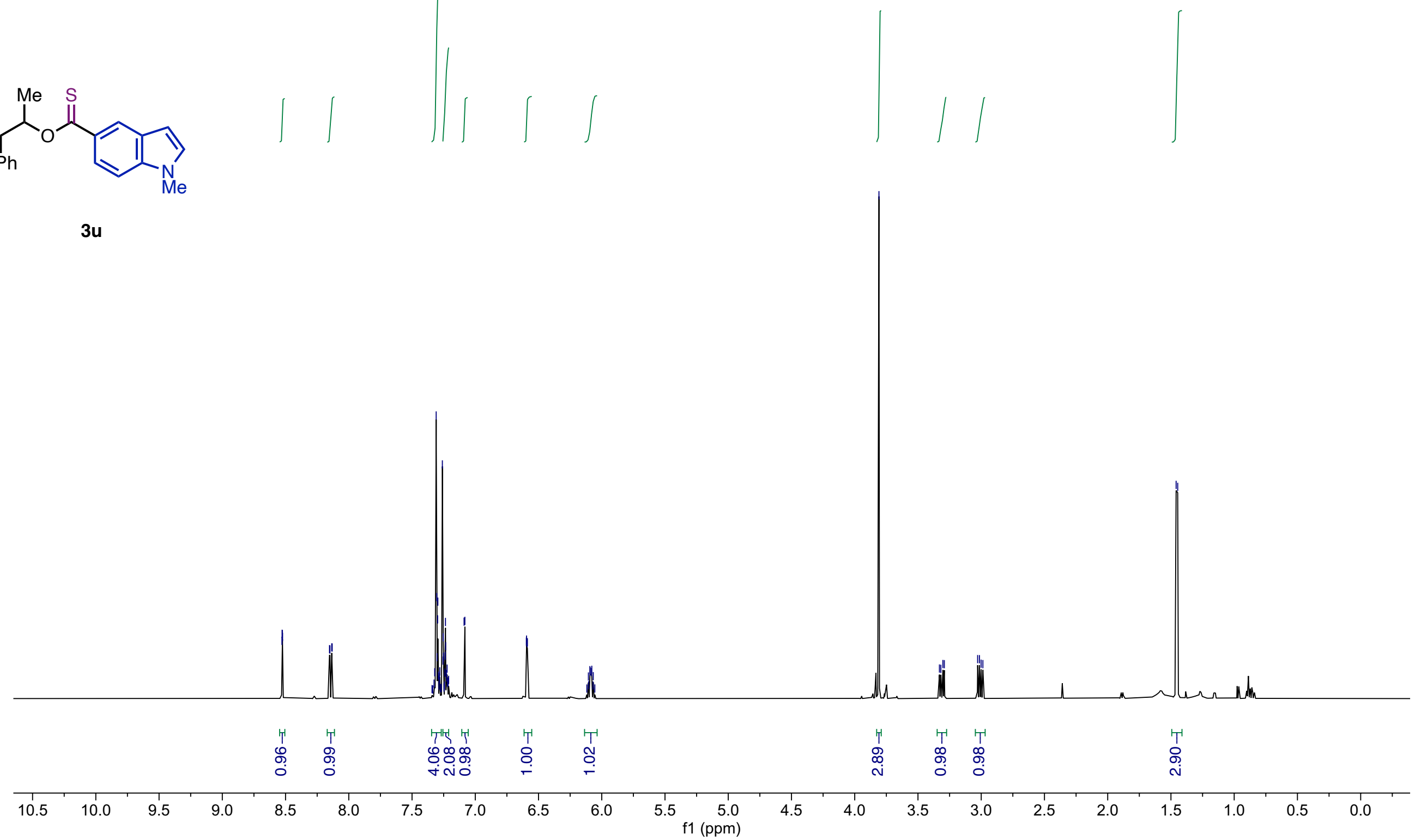
$126 \mathrm{MHz}, \mathrm{CDCl}_{3}$<smiles></smiles>

$3 \mathbf{u}$

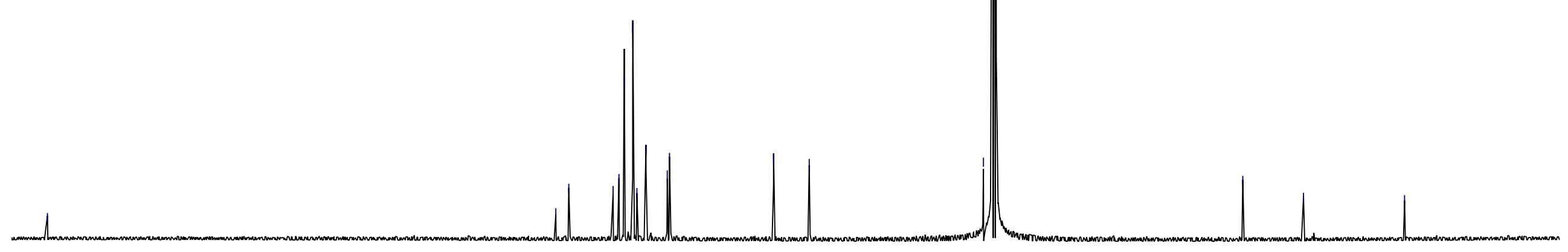


$400 \mathrm{MHz}, \mathrm{CDCl}_{3}$<smiles>CCCCCC(=S)OCCCc1ccccc1</smiles>

$3 v$

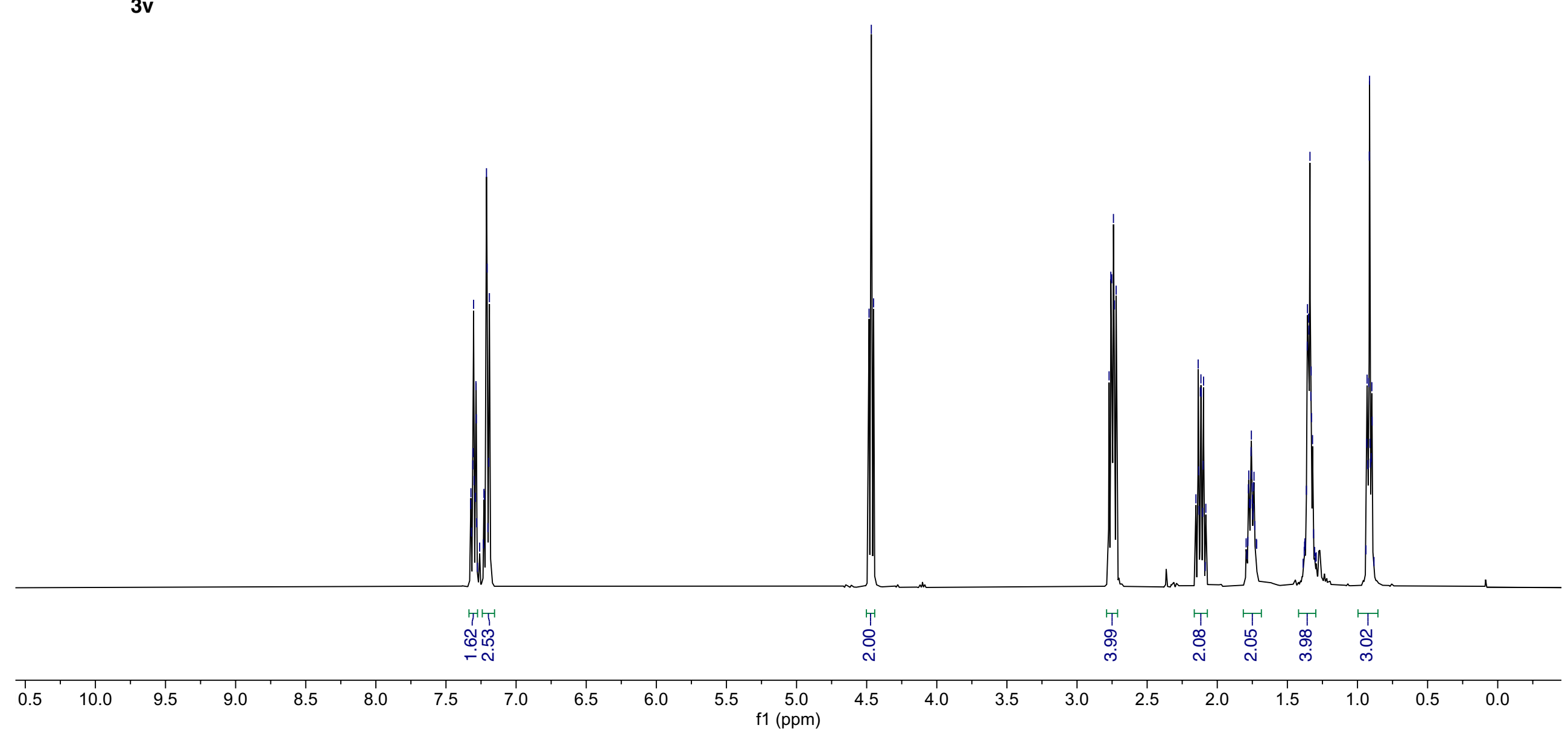


$101 \mathrm{MHz}, \mathrm{CDCl}_{3}$<smiles>CCCCCC(=S)OCCCc1ccccc1</smiles>

$3 v$

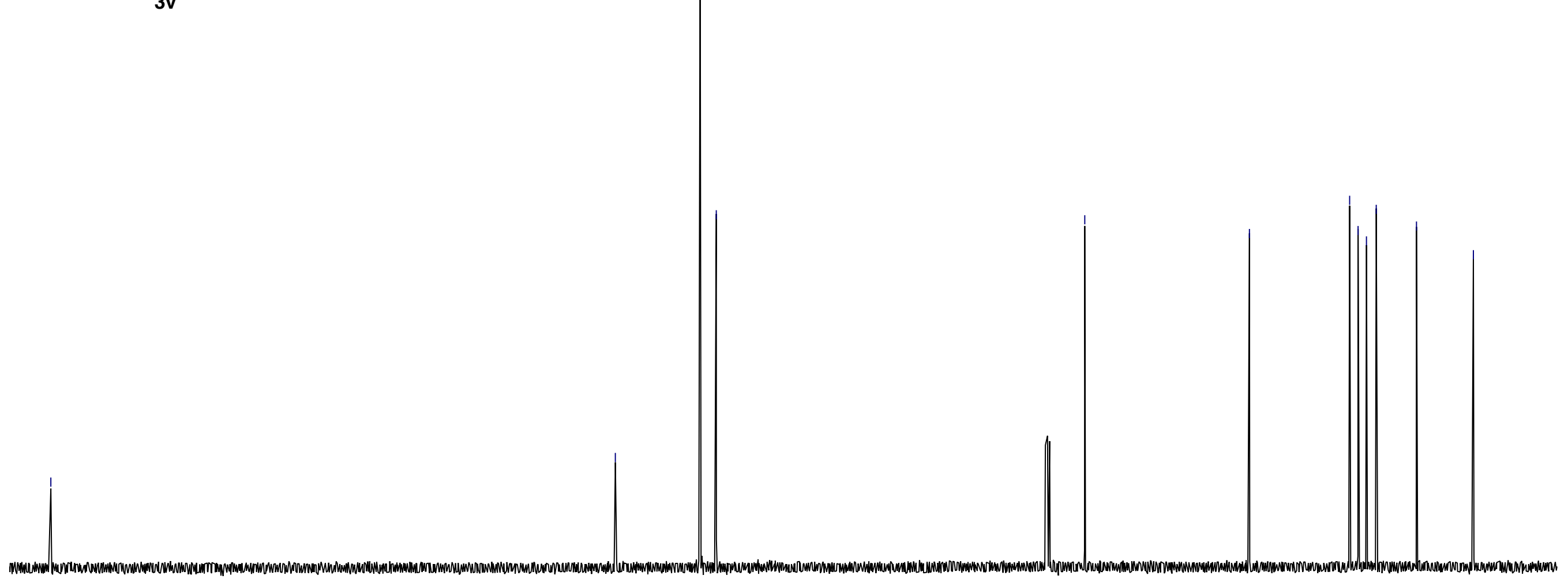


$500 \mathrm{MHz}, \mathrm{CDCl}_{3}$

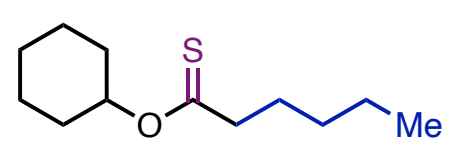

$3 w$
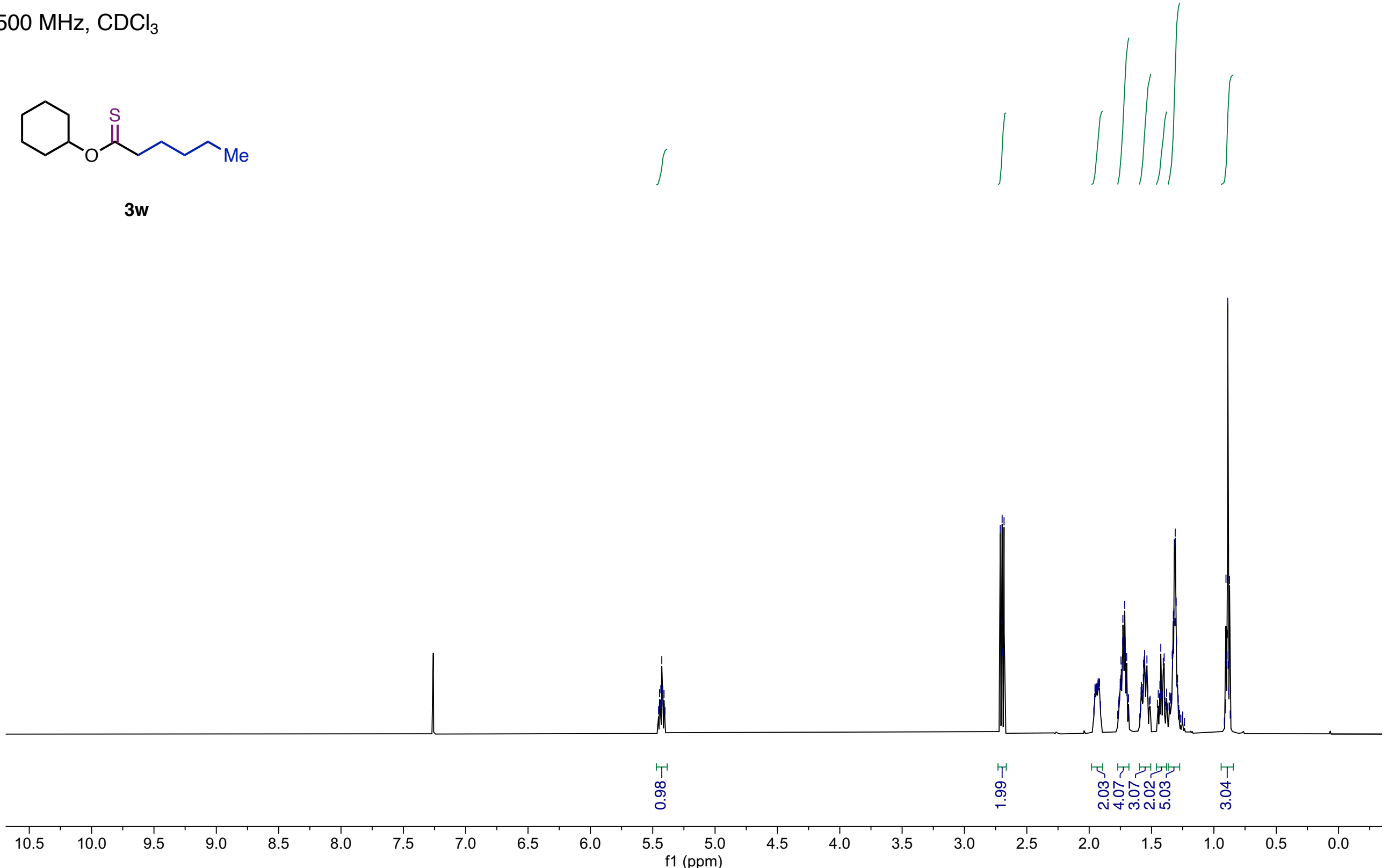
$126 \mathrm{MHz}, \mathrm{CDCl}_{3}$

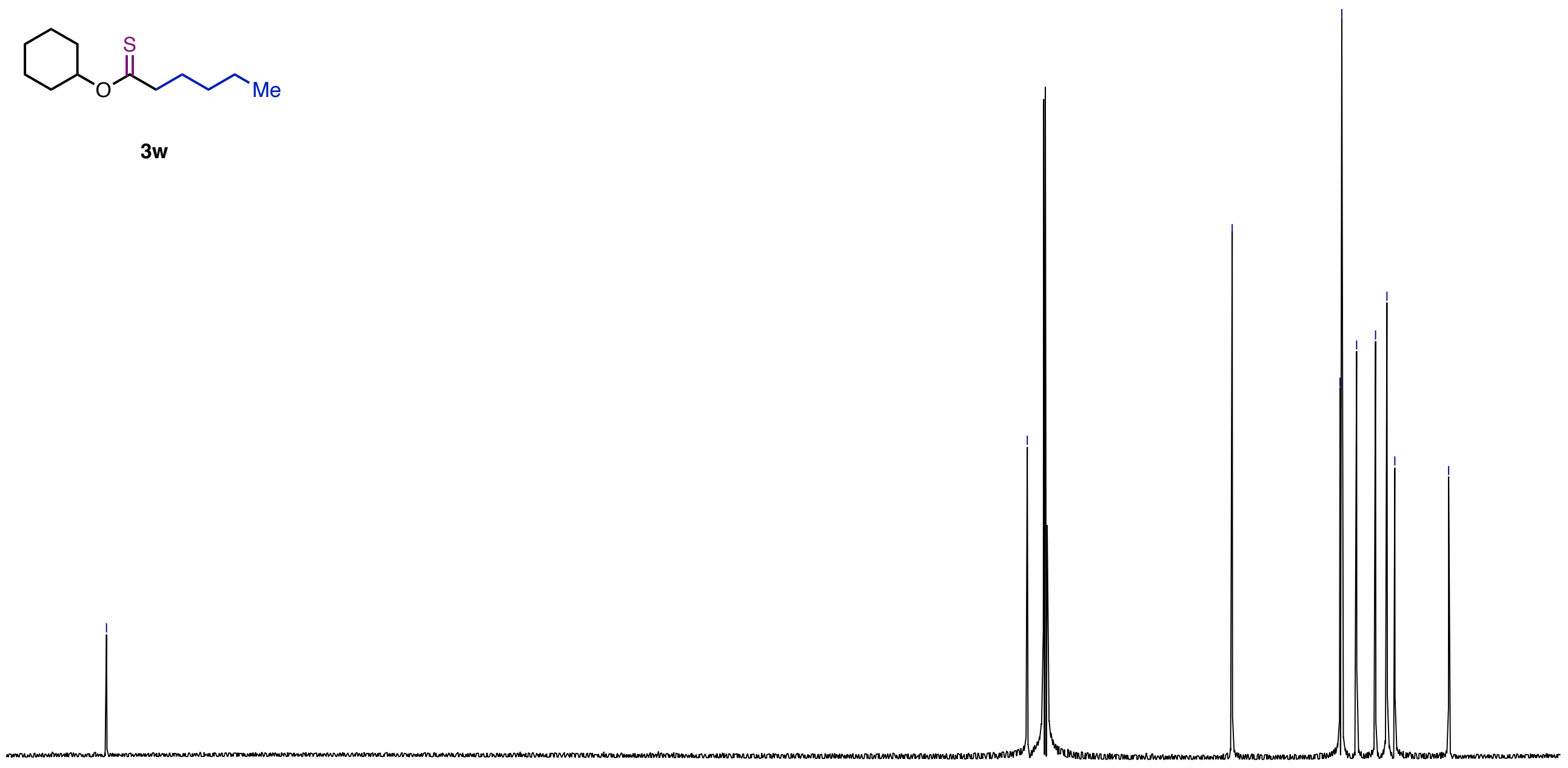


$500 \mathrm{MHz}, \mathrm{CDCl}_{3}$
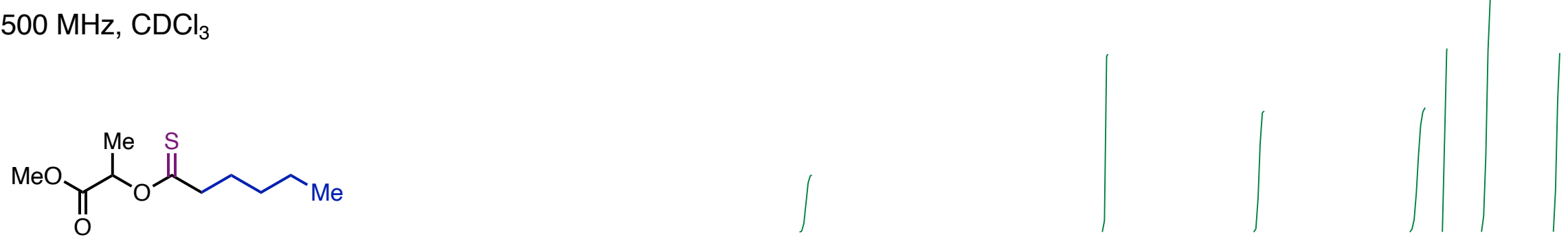

$3 x$

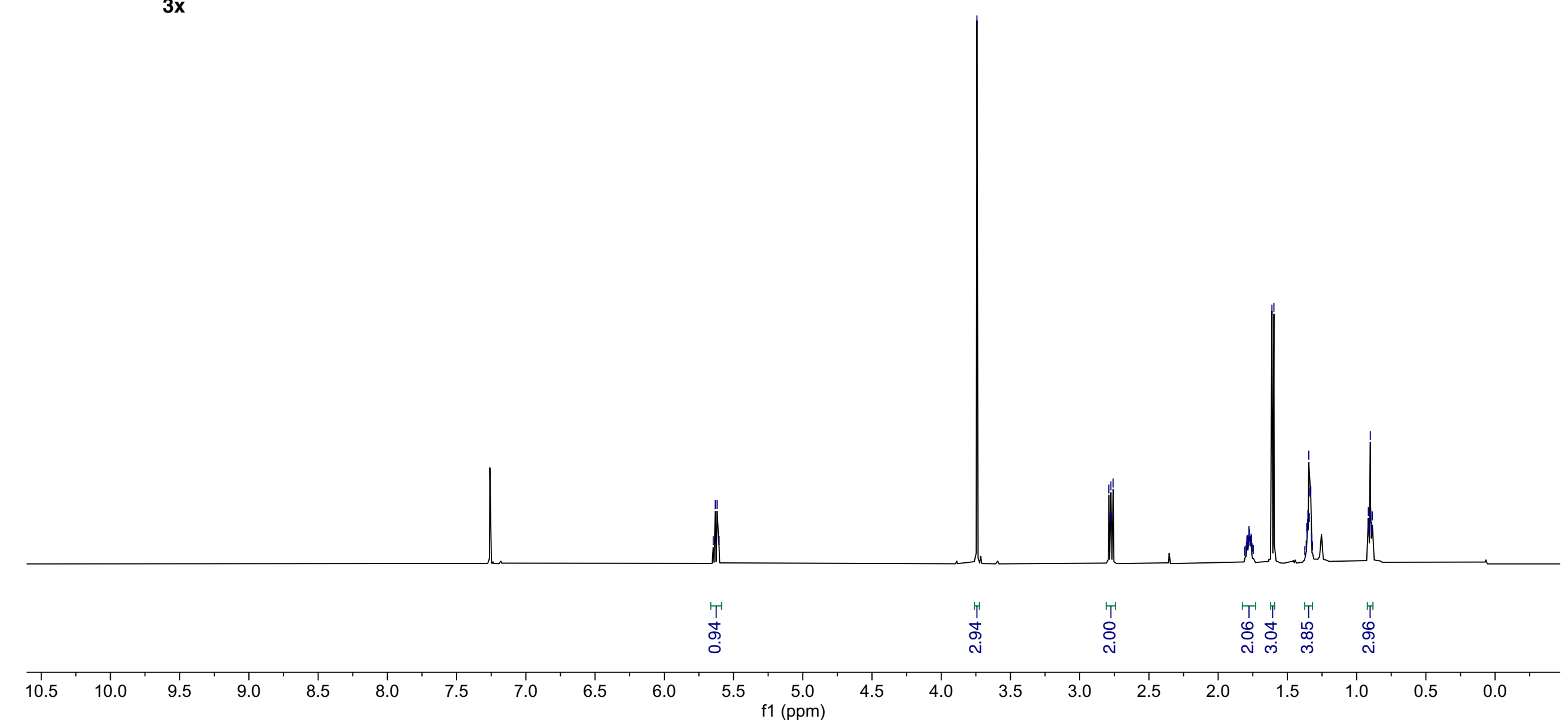


$126 \mathrm{MHz}, \mathrm{CDCl}_{3}$

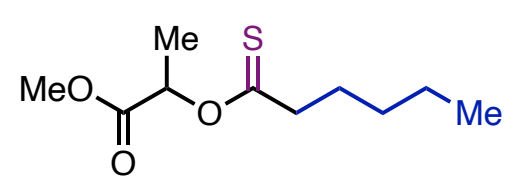

$3 \mathbf{x}$

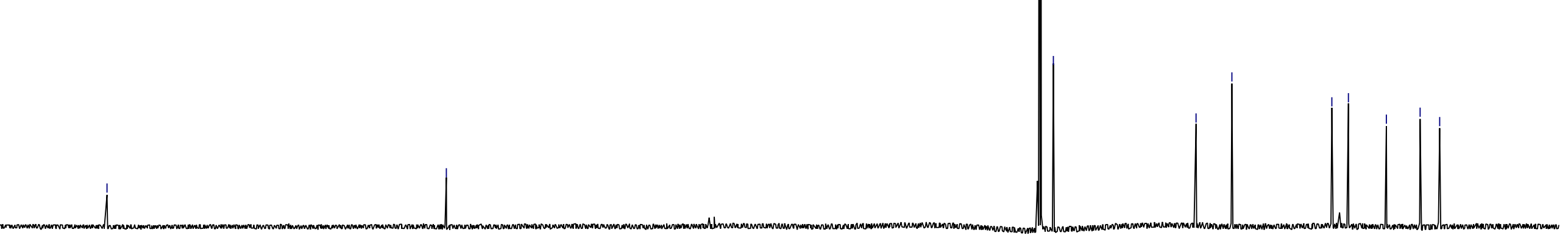


$500 \mathrm{MHz}, \mathrm{CDCl}_{3}$
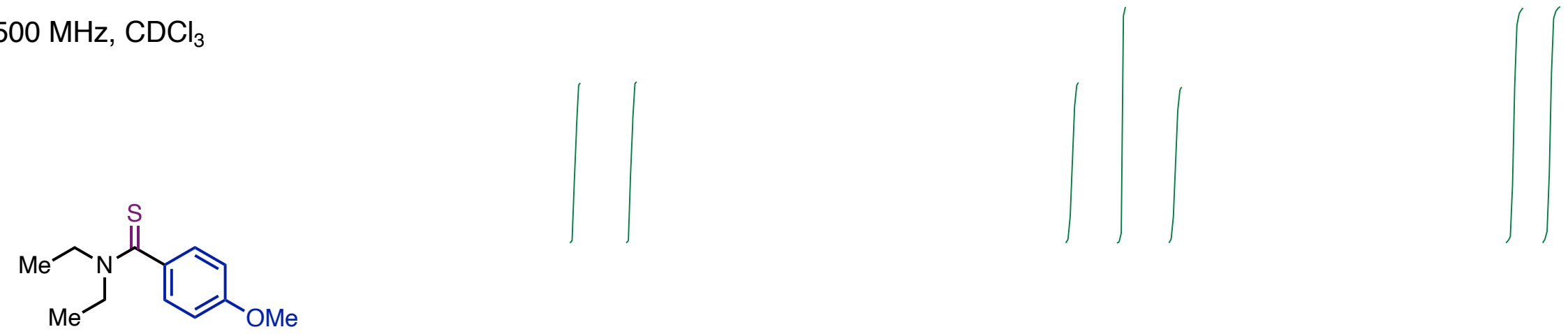

$5 a$

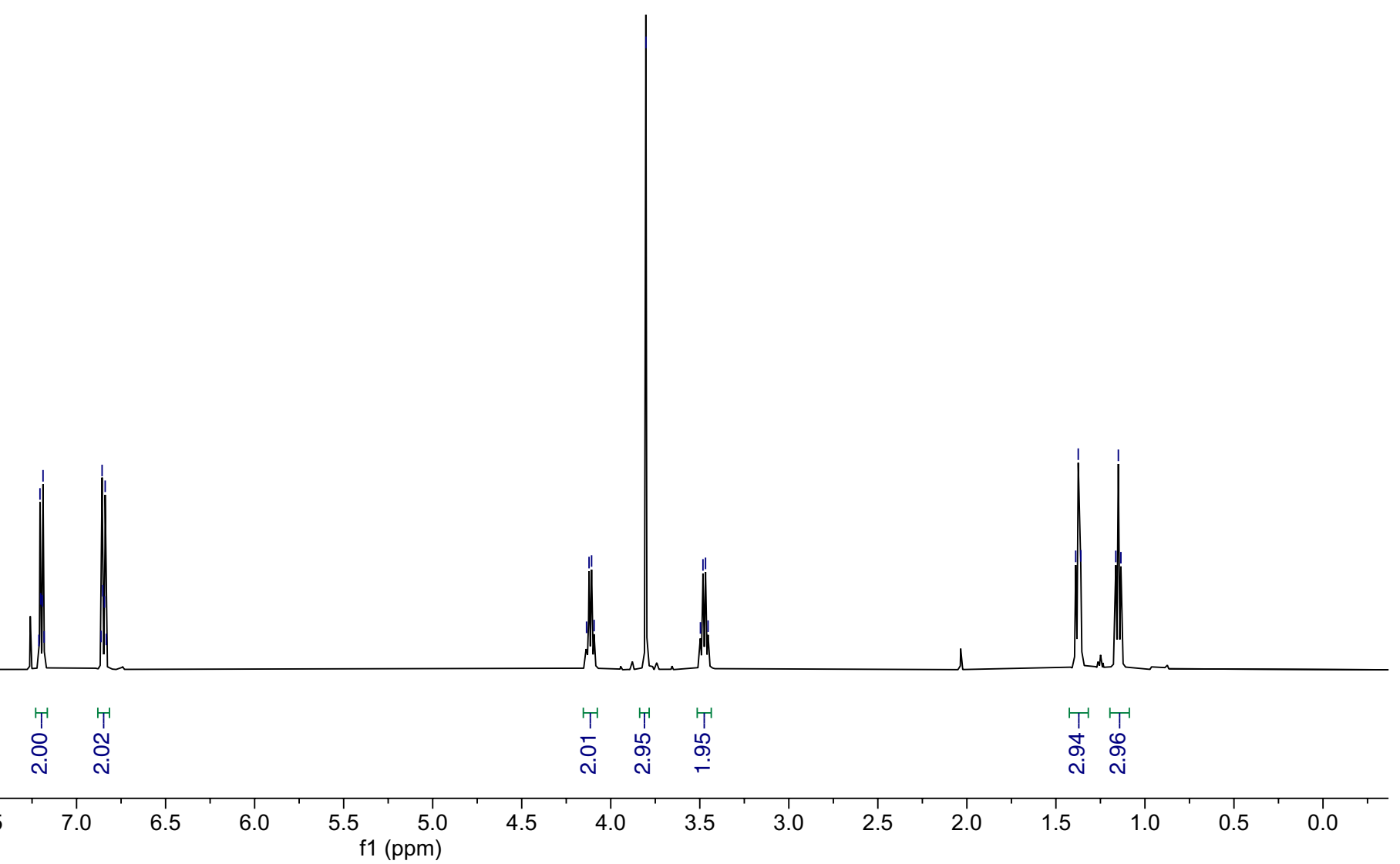


$126 \mathrm{MHz}, \mathrm{CDCl}_{3}$
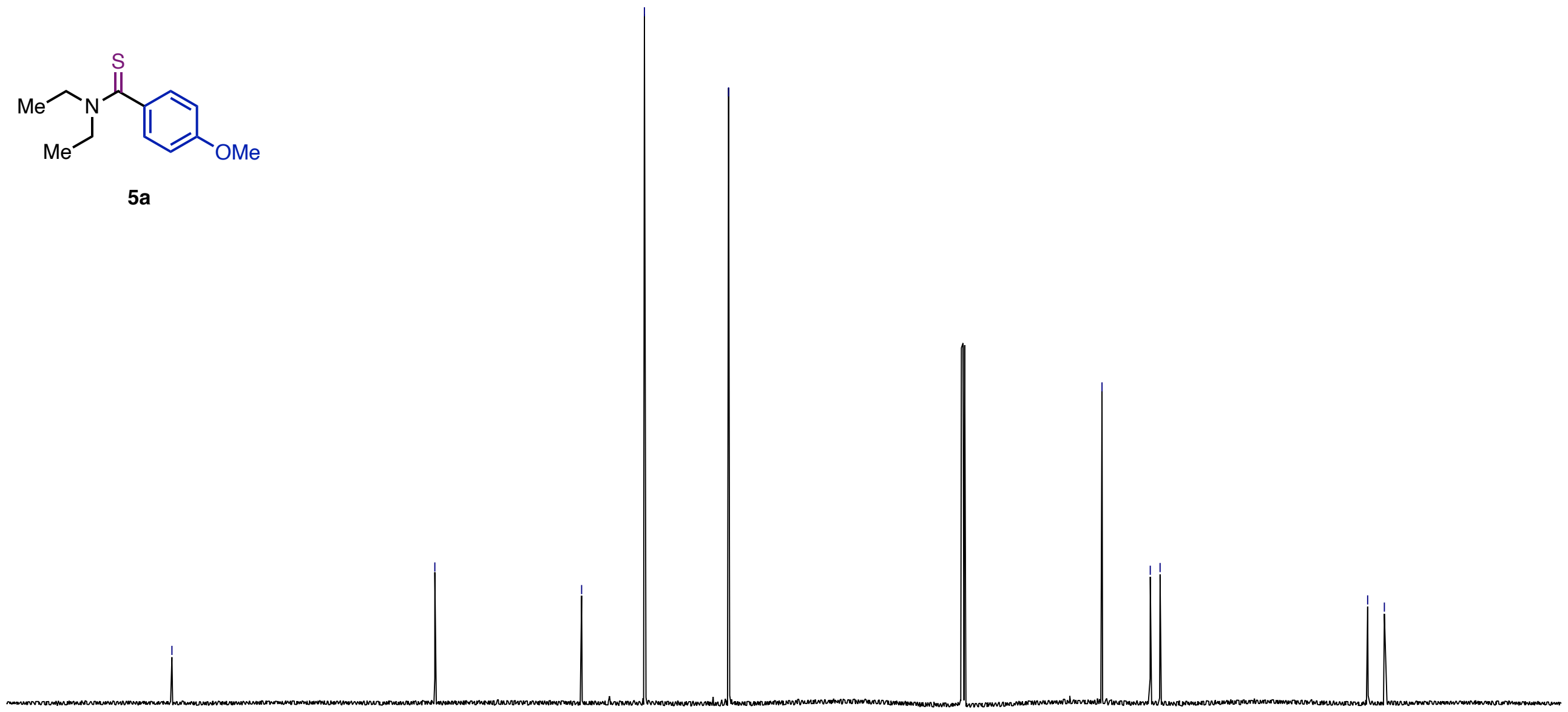
$500 \mathrm{MHz}, \mathrm{CDCl}_{3}$
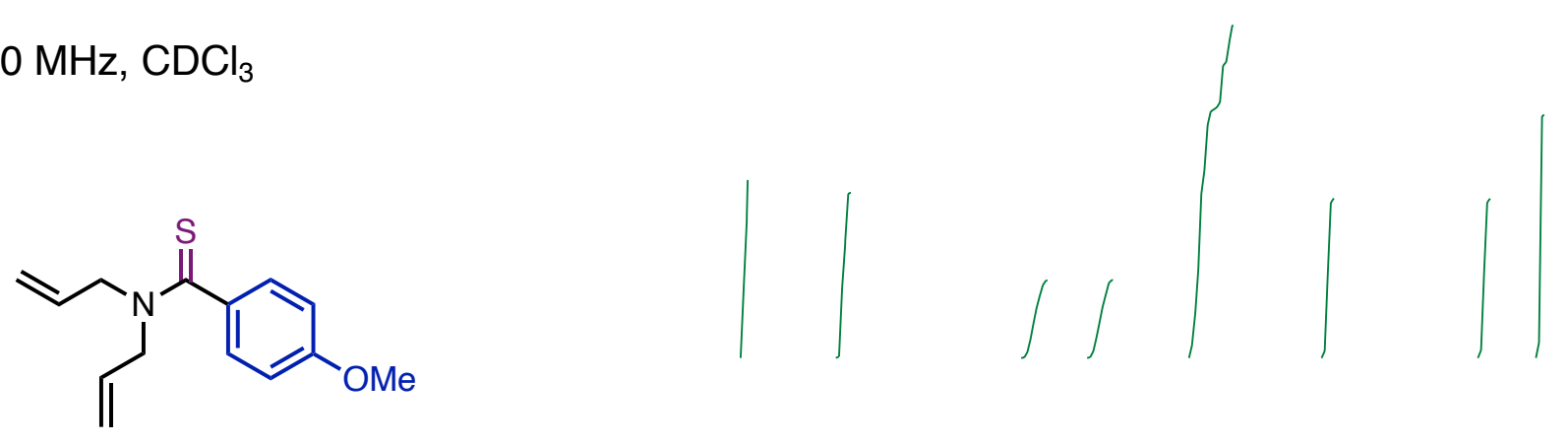

$5 b$

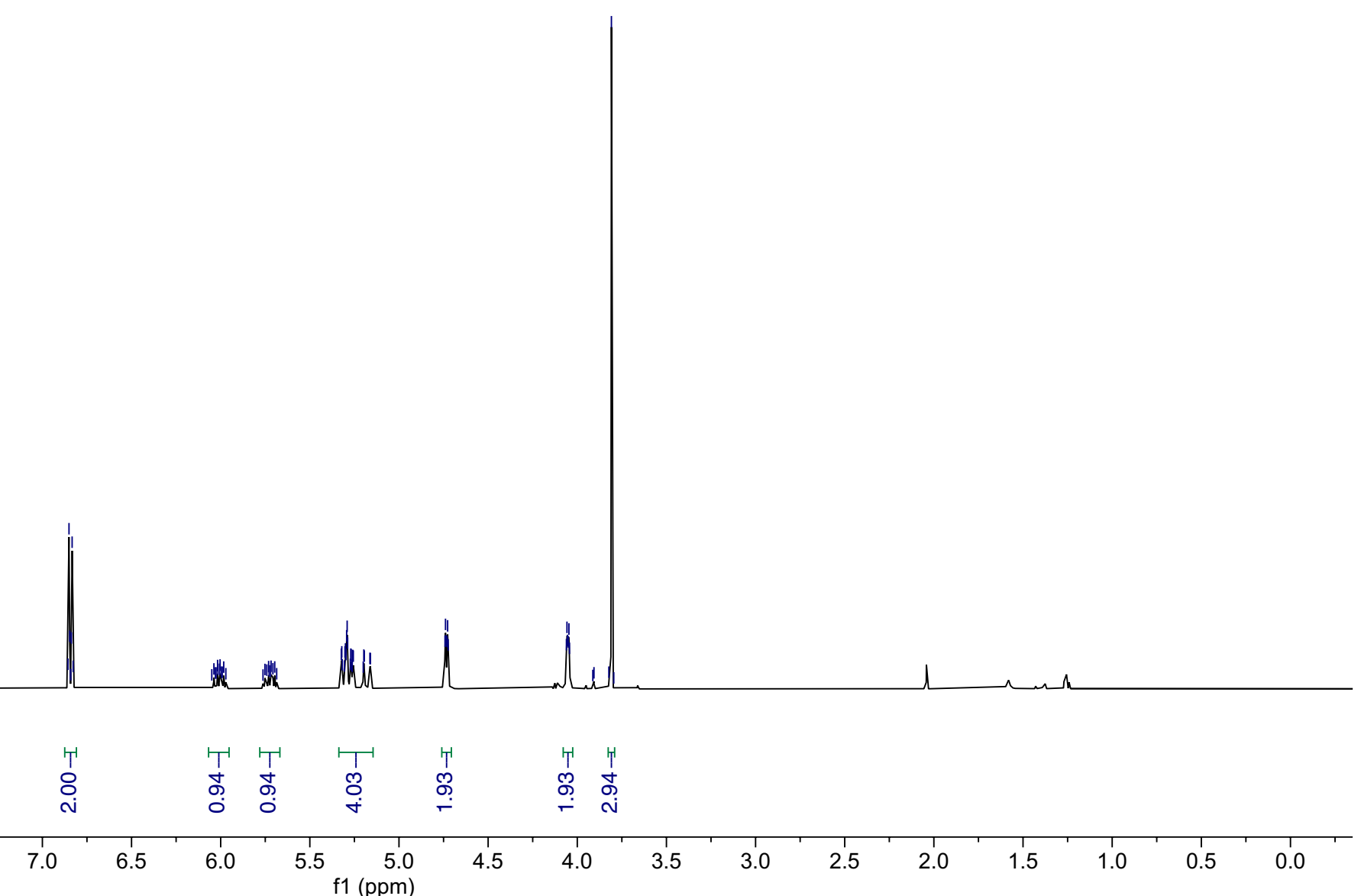




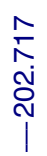

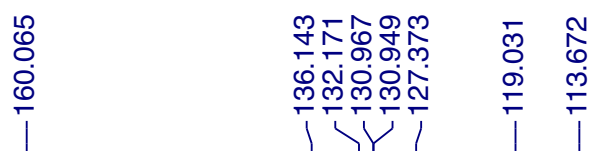

$126 \mathrm{MHz}, \mathrm{CDCl}_{3}$<smiles>C=CCN(CC=C)C(=S)c1ccc(OC)cc1</smiles>

$5 b$

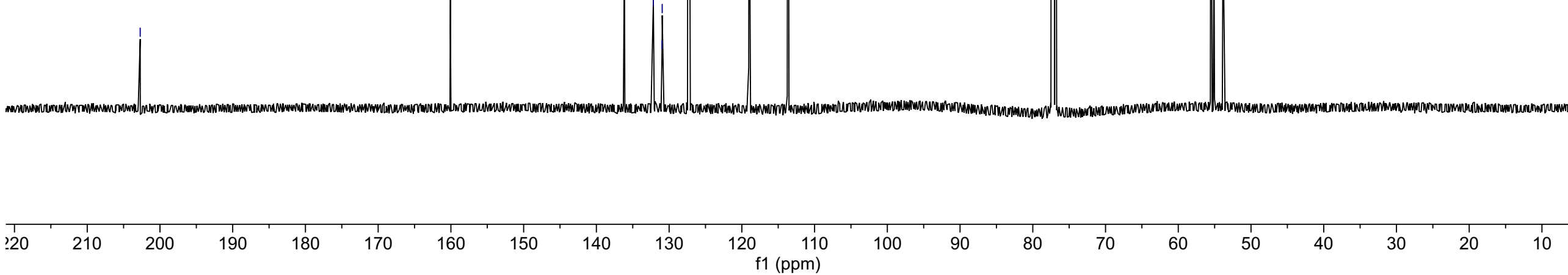

S92 


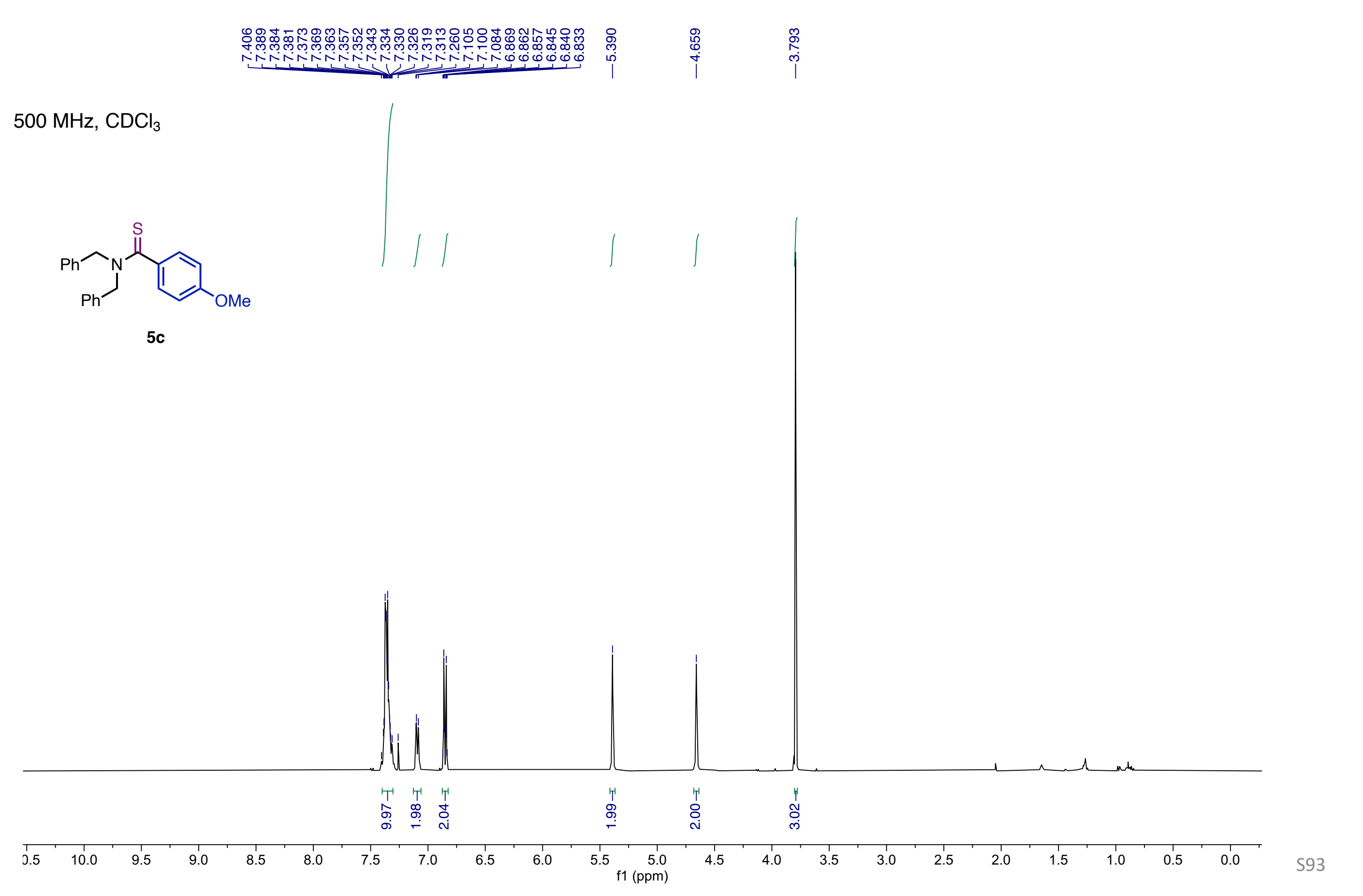


$\infty$
$\infty$
$\infty$
$\stackrel{0}{0}$
$\stackrel{N}{\mid}$

\begin{tabular}{l}
$\infty$ \\
0 \\
0 \\
\hdashline \\
\hline
\end{tabular}

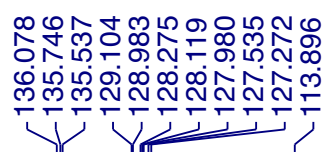

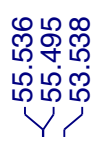

$126 \mathrm{MHz}, \mathrm{CDCl}_{3}$<smiles>COc1ccc(C(=S)N(Cc2ccccc2)Cc2ccccc2)cc1</smiles>

5c

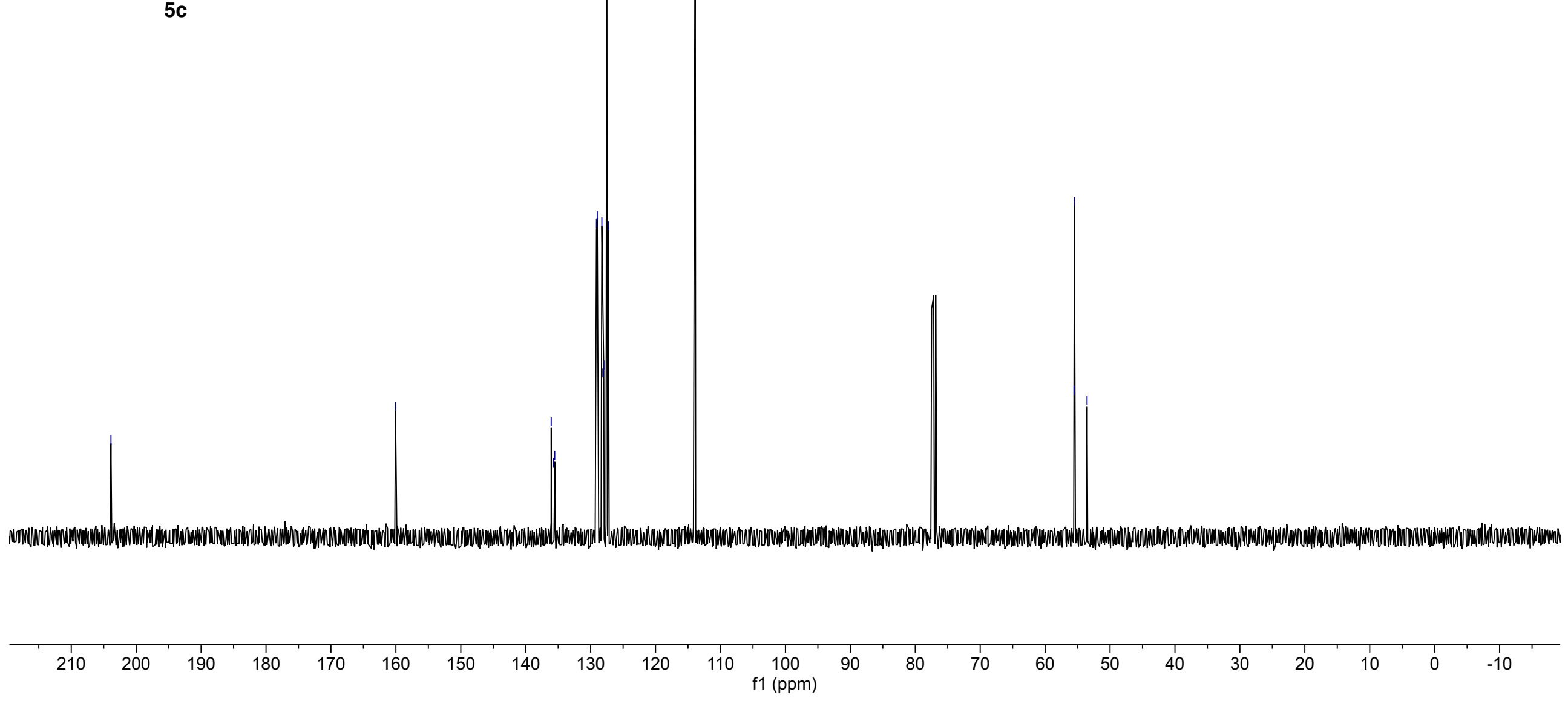

S94 
$500 \mathrm{MHz}, \mathrm{CDCl}_{3}$
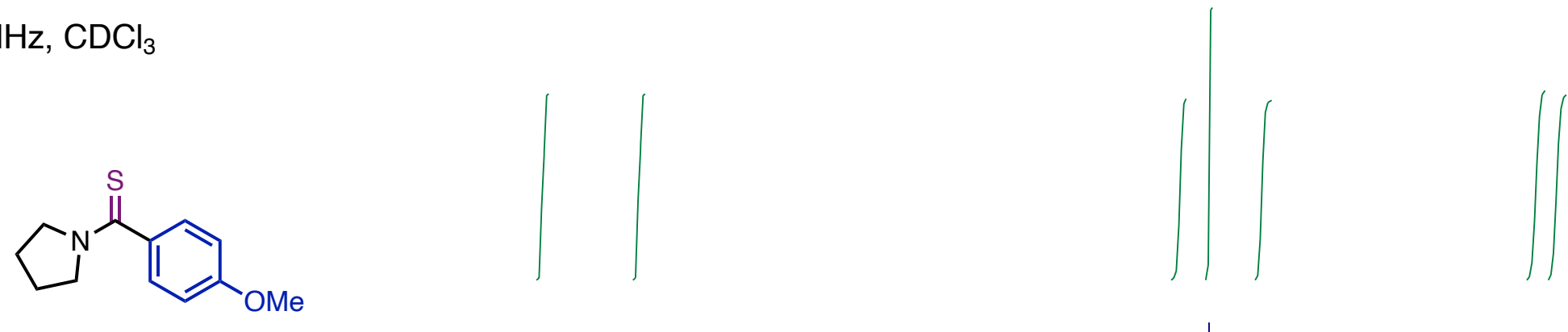

$5 d$

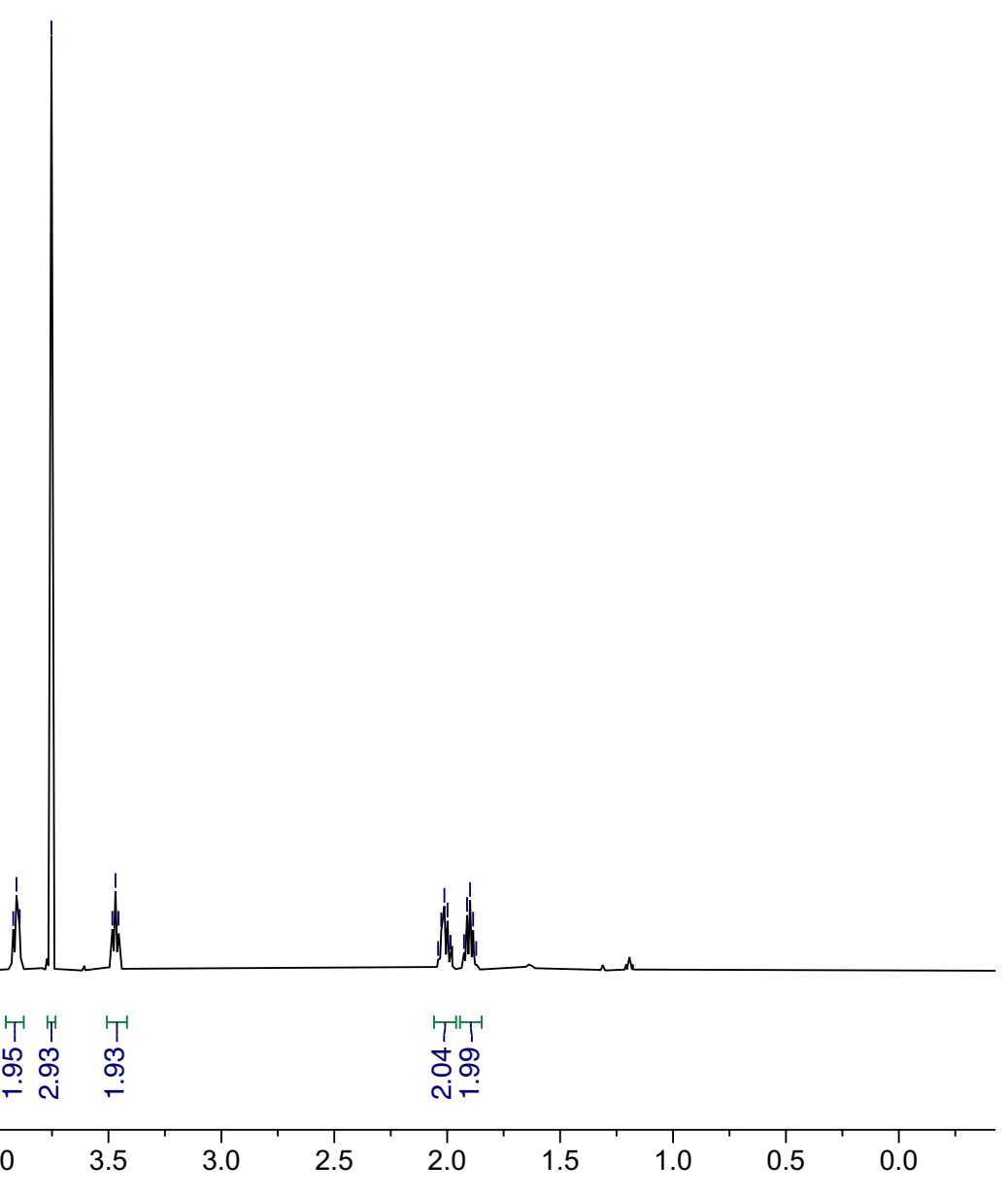


กั

\begin{tabular}{l}
0 \\
\multirow{2}{N}{} \\
0 \\
0 \\
\hline
\end{tabular}

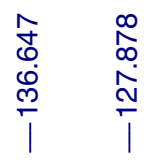

苫

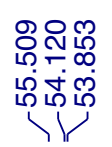

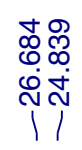

$126 \mathrm{MHz}^{\mathrm{CDCl}_{3}}$<smiles>COc1ccc(C(=S)N2CCCC2)cc1</smiles>

$5 d$
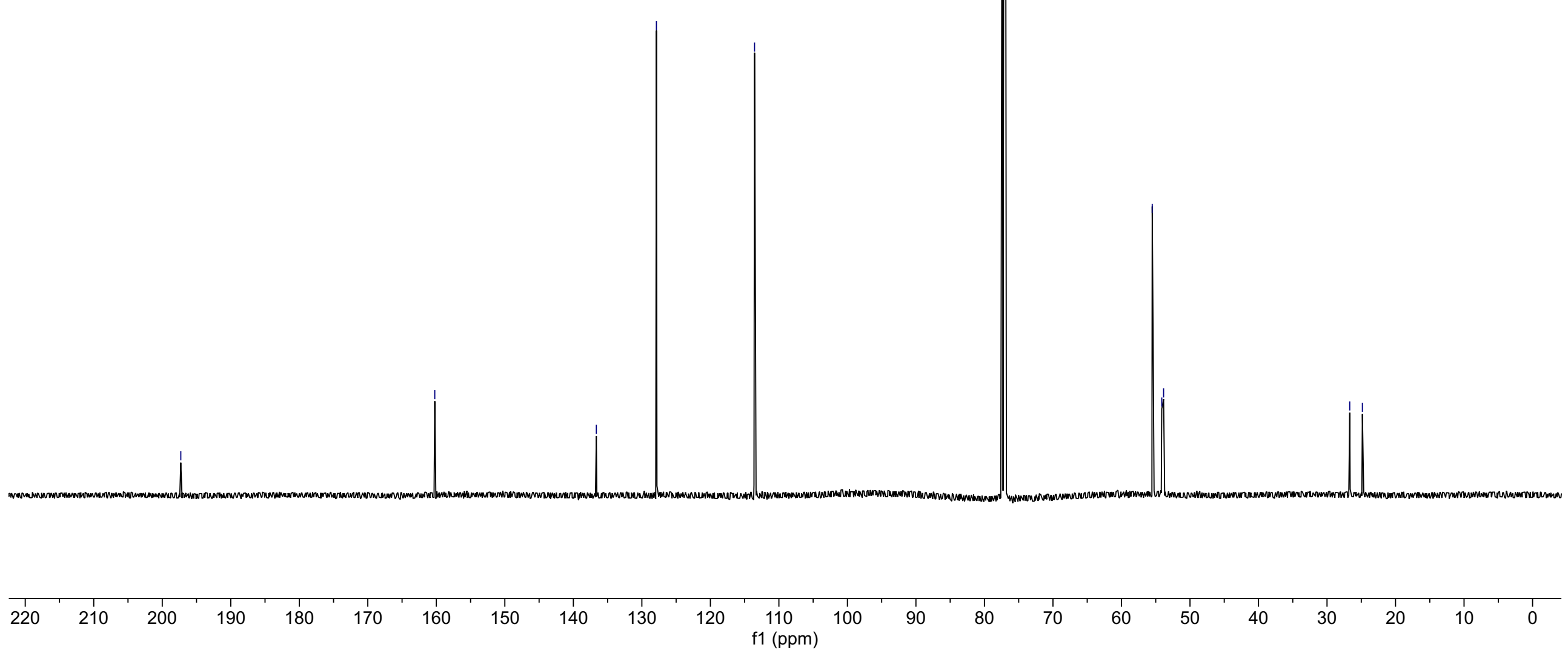

S96 
$\underbrace{n-1}$
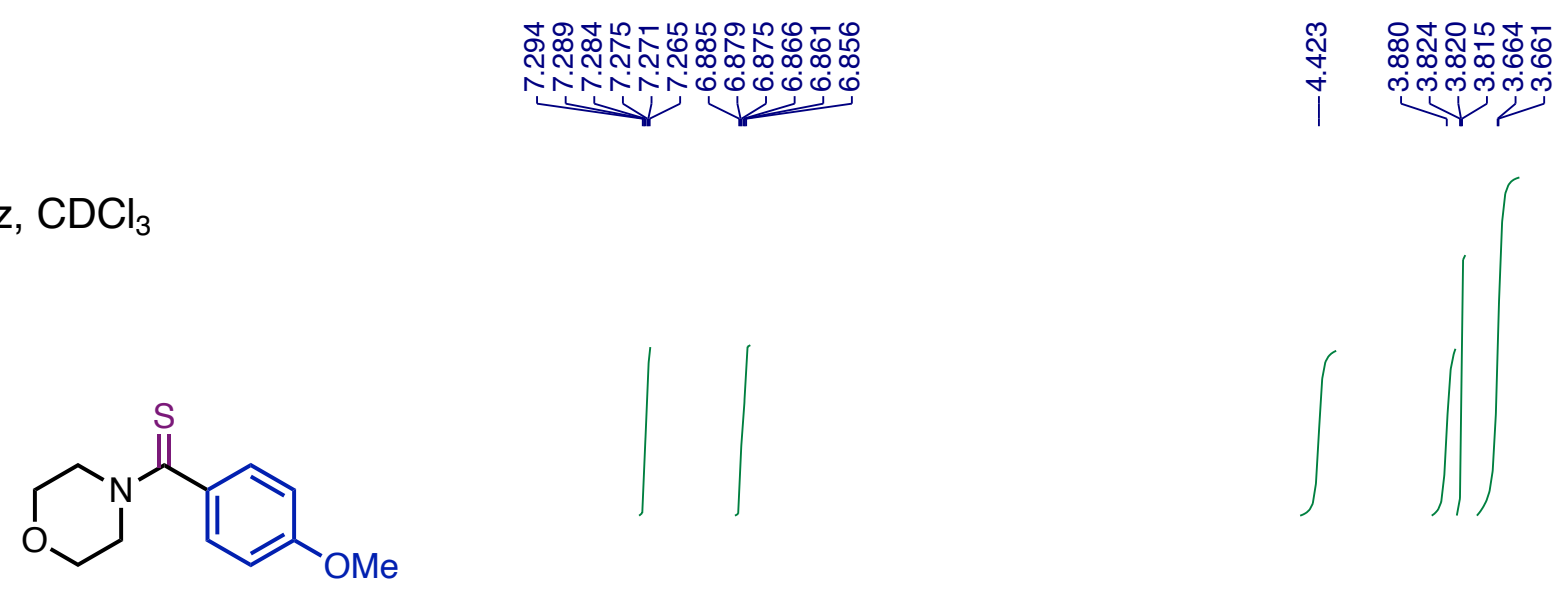

$5 e$

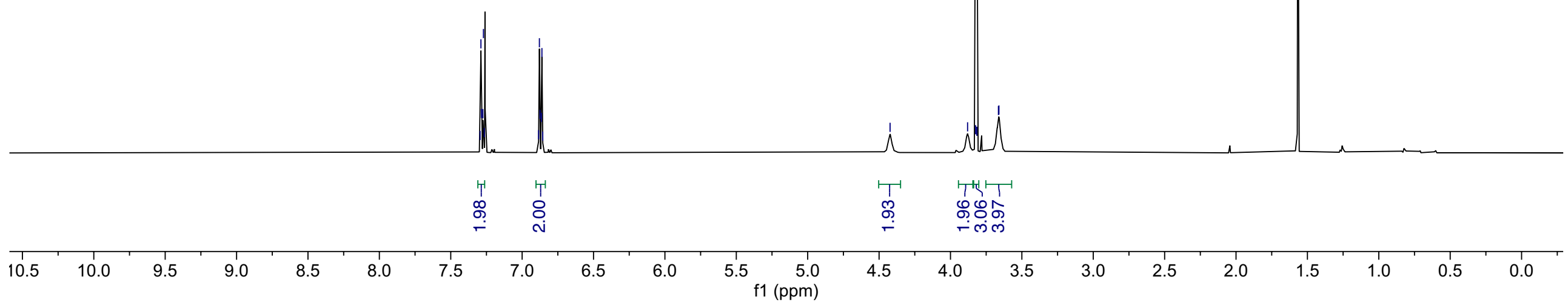

S97 
ヘิ

0
$\vdots$
0
0

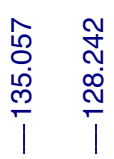

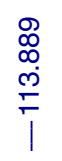

$\infty \bar{N}$
बूर
$0 \%$
60

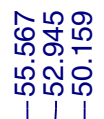

$126 \mathrm{MHz}, \mathrm{CDCl}_{3}$<smiles>COc1ccc(C(=S)N2CCOCC2)cc1</smiles>

$5 e$

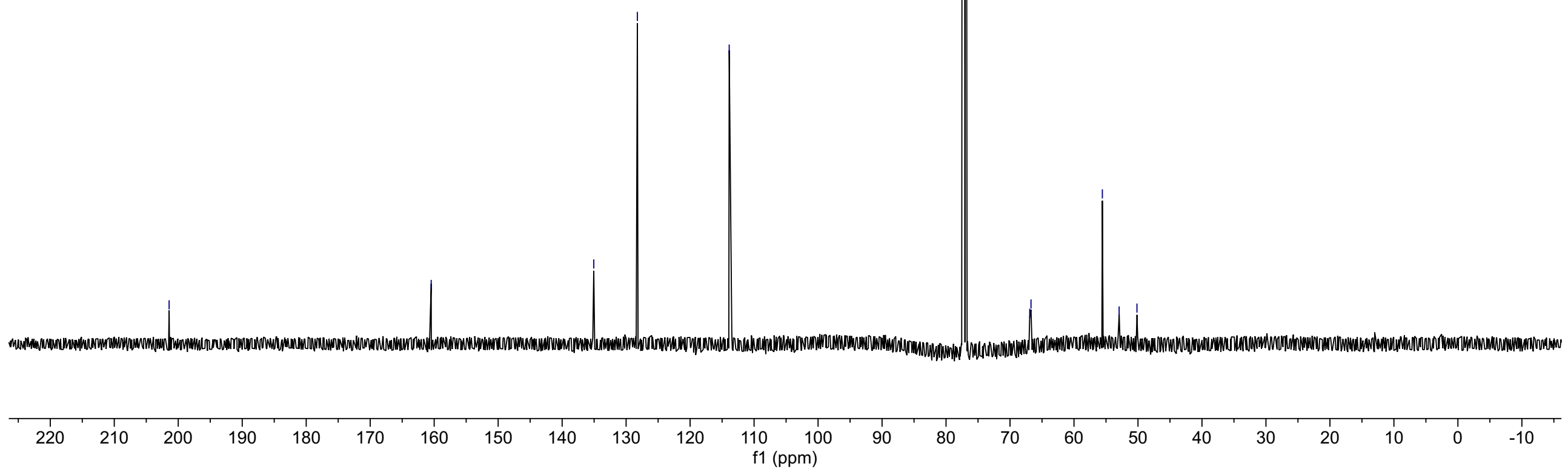

S98 
$400 \mathrm{MHz}, \mathrm{CDCl}_{3}$
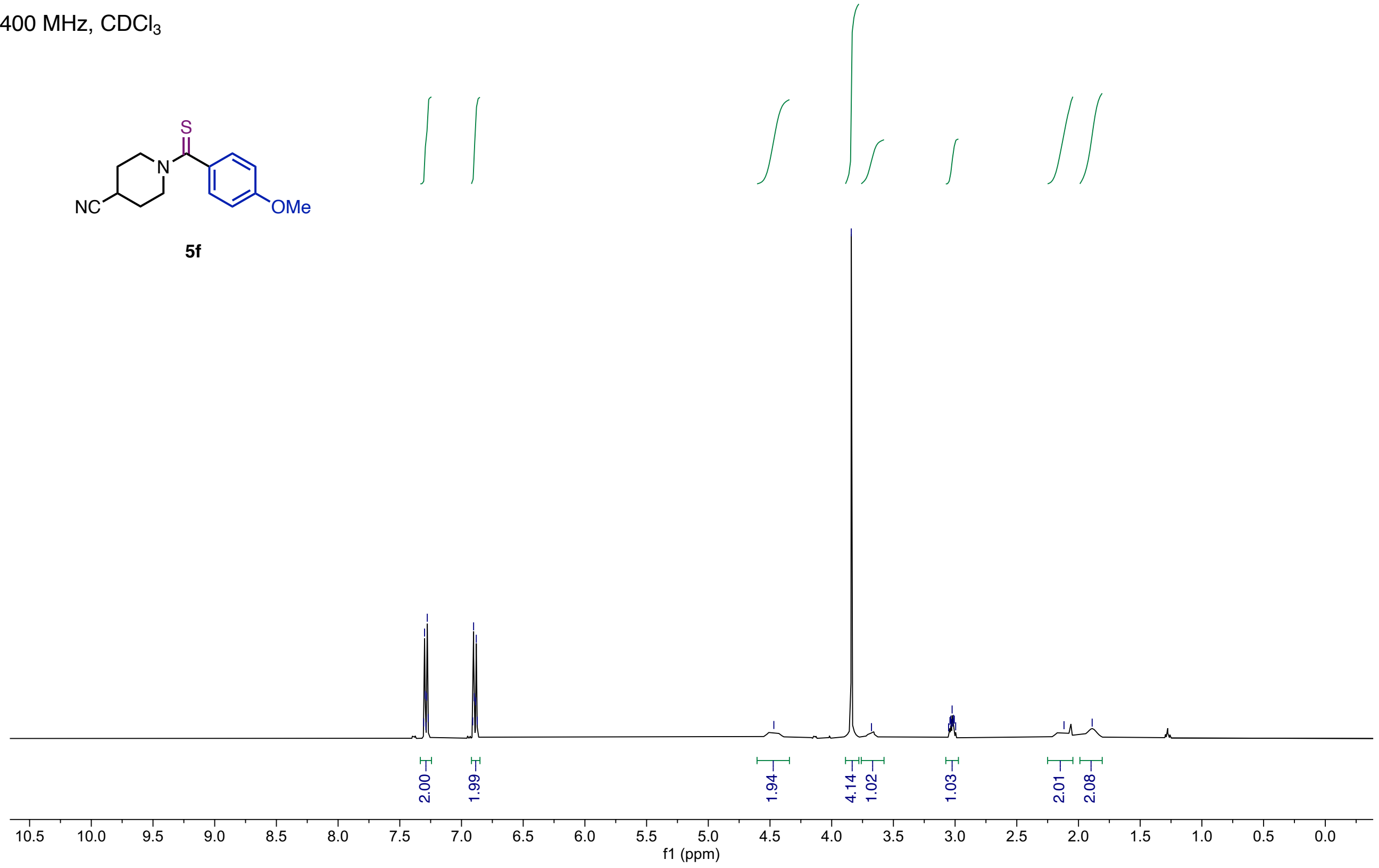

$500 \mathrm{MHz}, \mathrm{CDCl}_{3}$
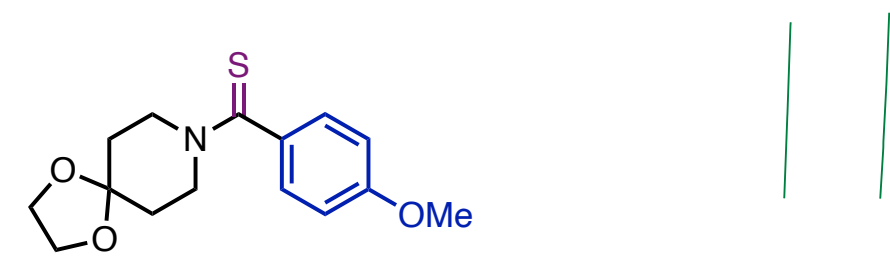

讨

$5 g$
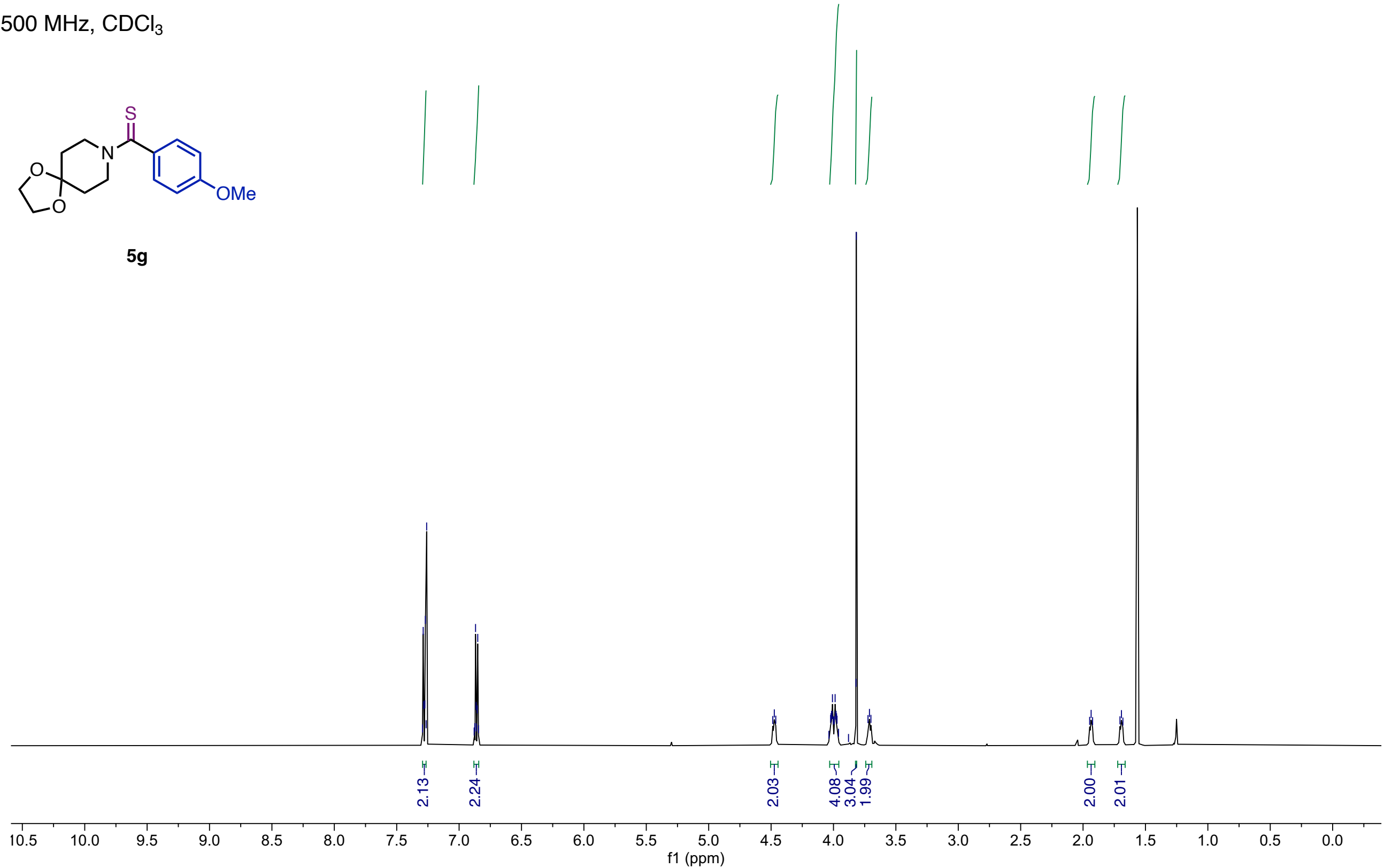
$126 \mathrm{MHz}, \mathrm{CDCl}_{3}$

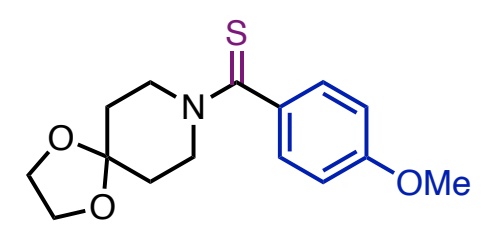




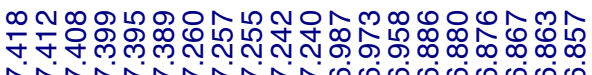

$500 \mathrm{MHz}, \mathrm{CDCl}_{3}$

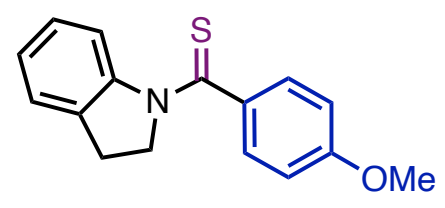

$5 \mathrm{~h}$

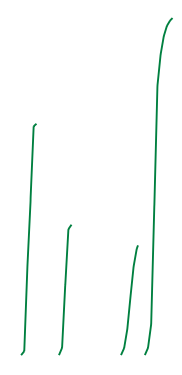

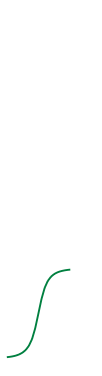

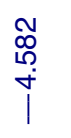

$\underset{\substack{\infty \\ \infty}}{\infty}$

نூ

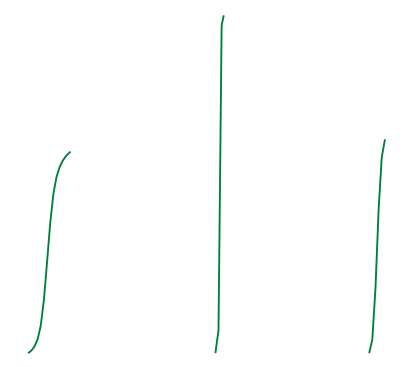


$126 \mathrm{MHz}, \mathrm{CDCl}_{3}$

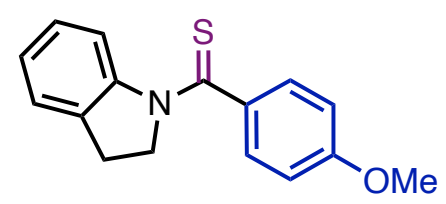

$5 \mathrm{~h}$

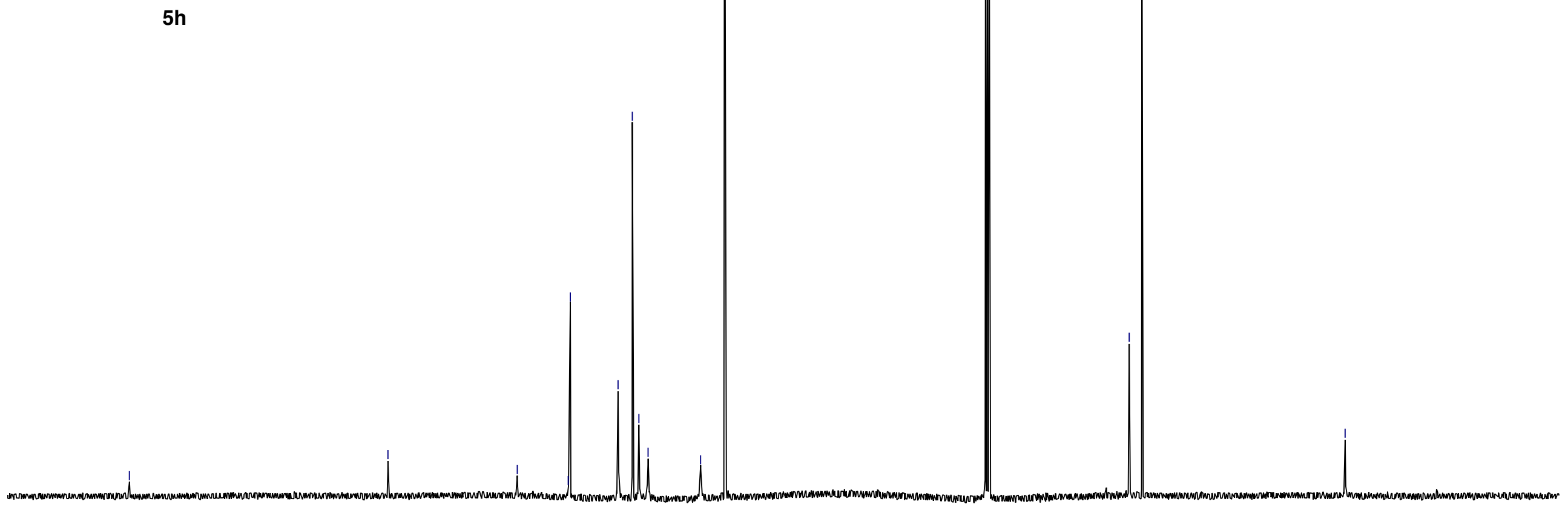

$210 \quad 200$ 


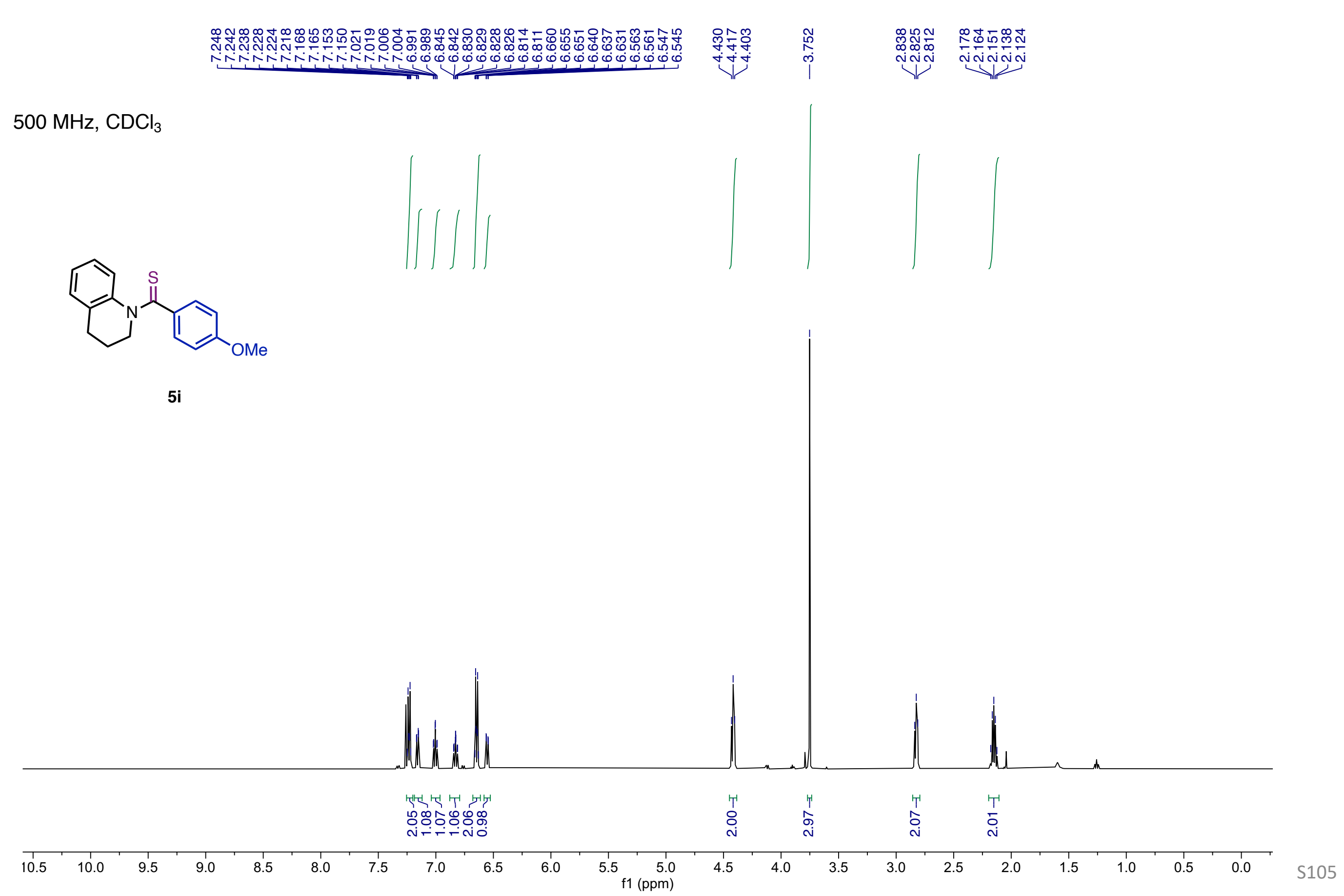




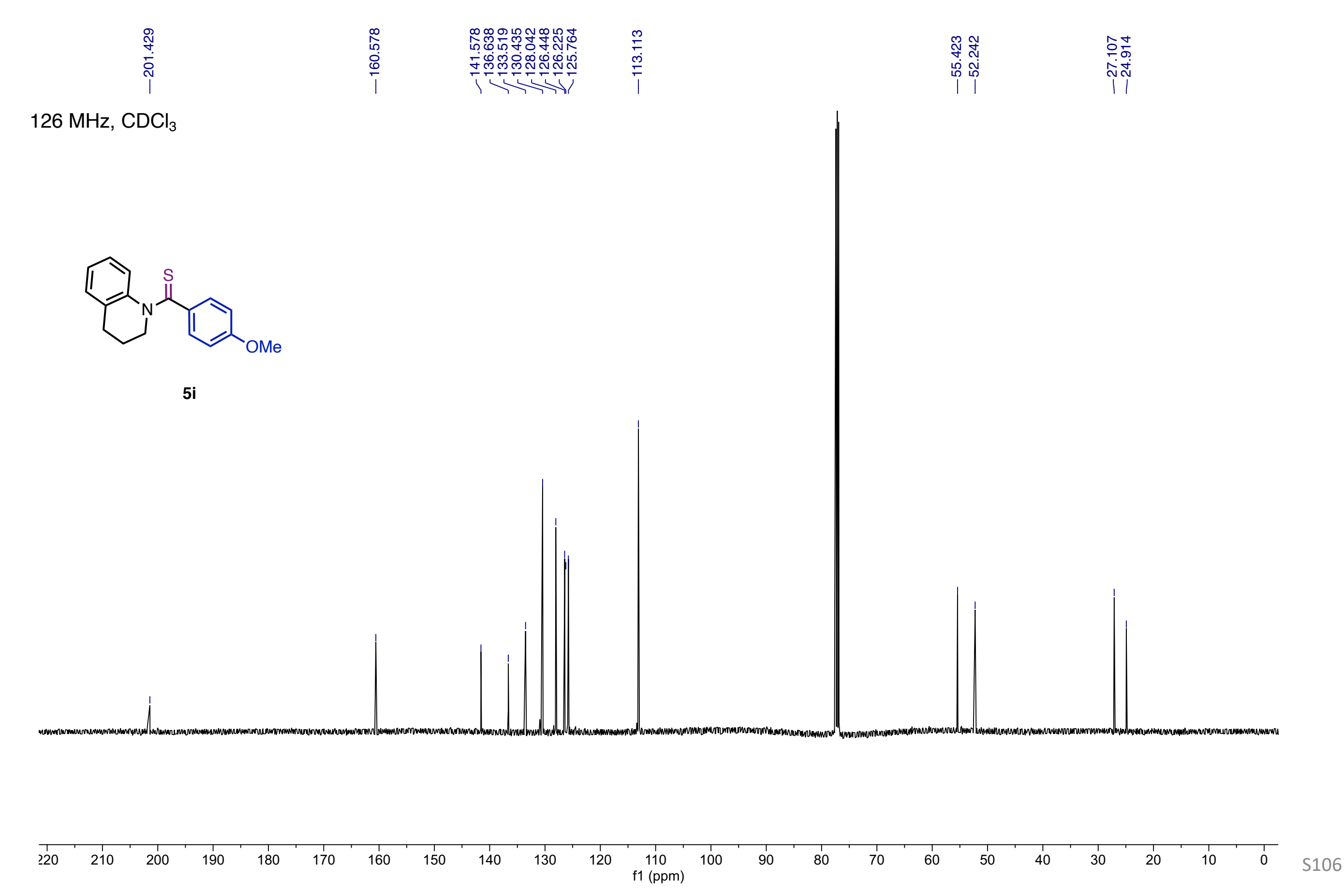


$400 \mathrm{MHz}, \mathrm{CDCl}_{3}$
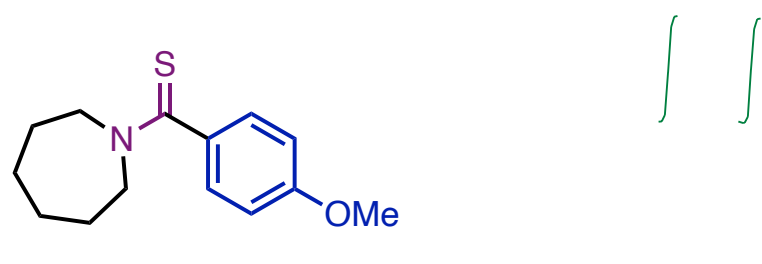

$5 j$

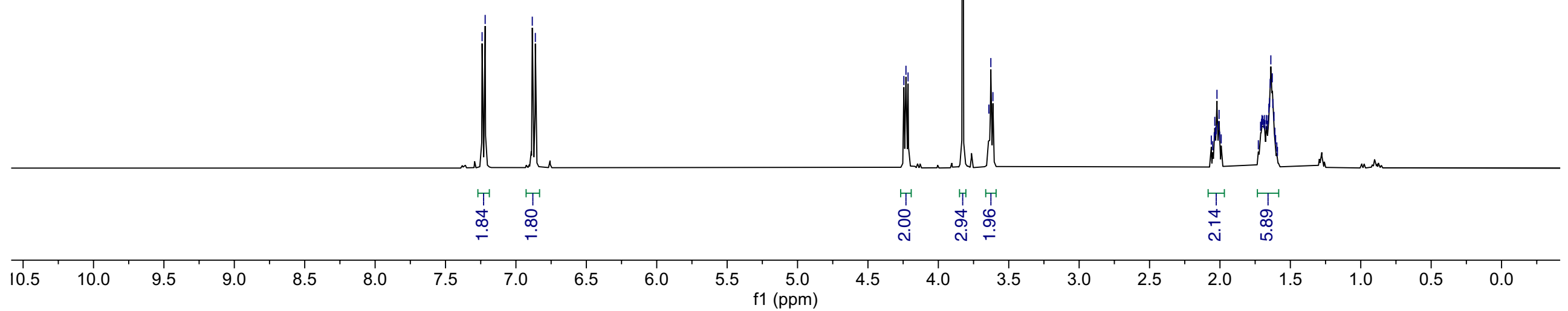


$400 \mathrm{MHz}, \mathrm{CDCl}_{3}$

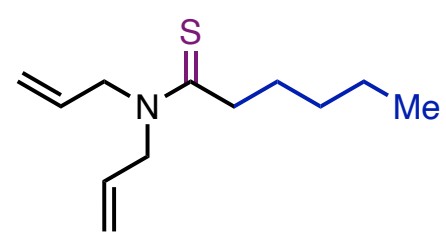

$5 k$
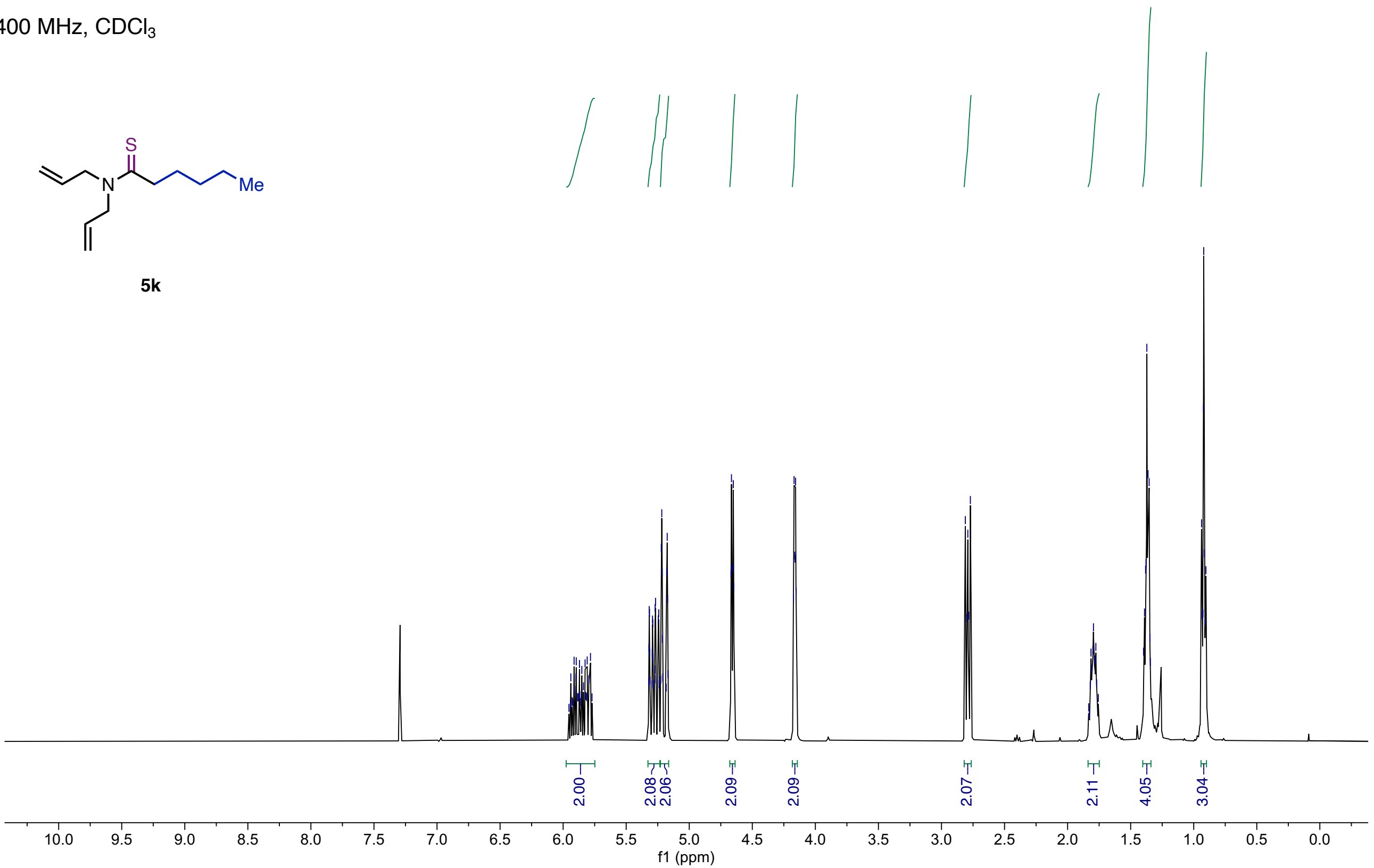
$\infty$
$\stackrel{0}{0}$
$\stackrel{N}{1}$
I

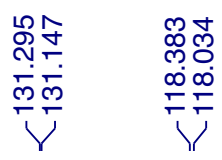

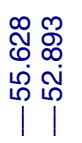

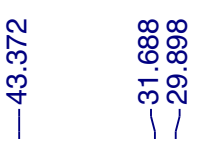

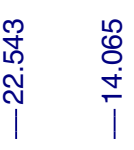

$101 \mathrm{MHz}, \mathrm{CDCl}_{3}$<smiles>C=CCN(CC=C)C(=S)CCCCC</smiles>

$5 k$

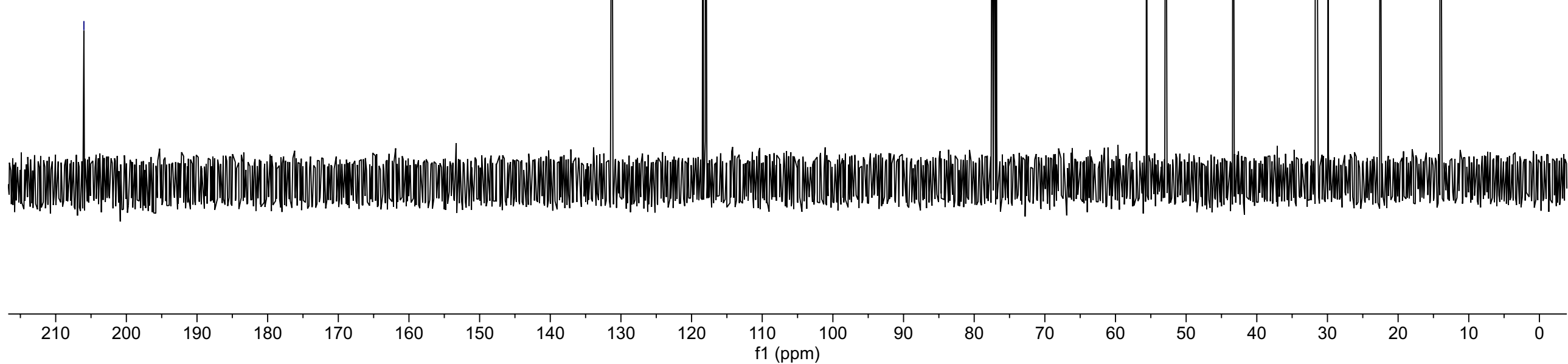

S110 
$400 \mathrm{MHz}, \mathrm{CDCl}_{3}$
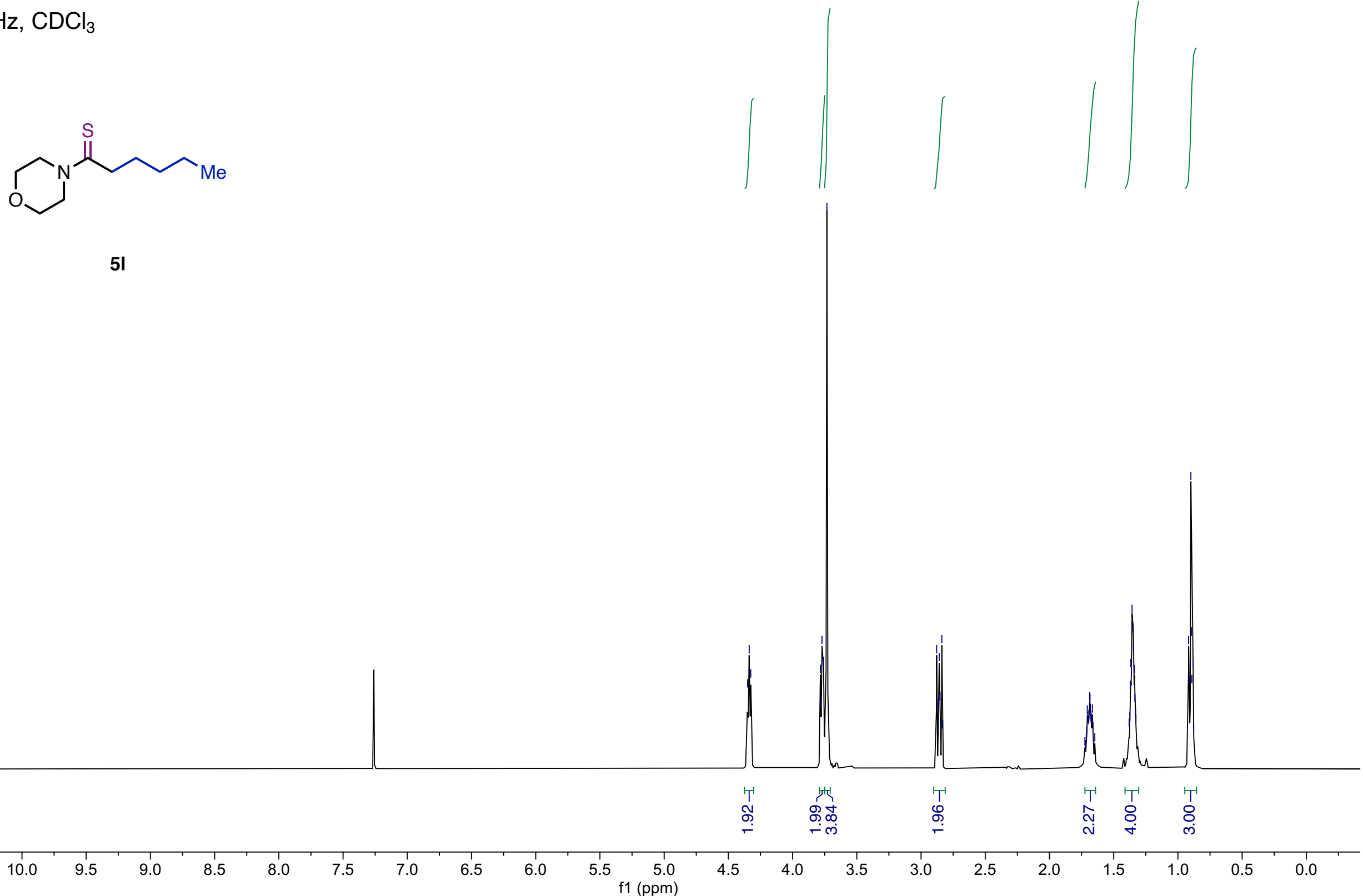

51 
$101 \mathrm{MHz}, \mathrm{CDCl}_{3}$

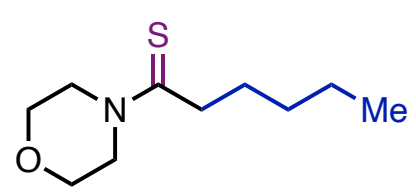

51

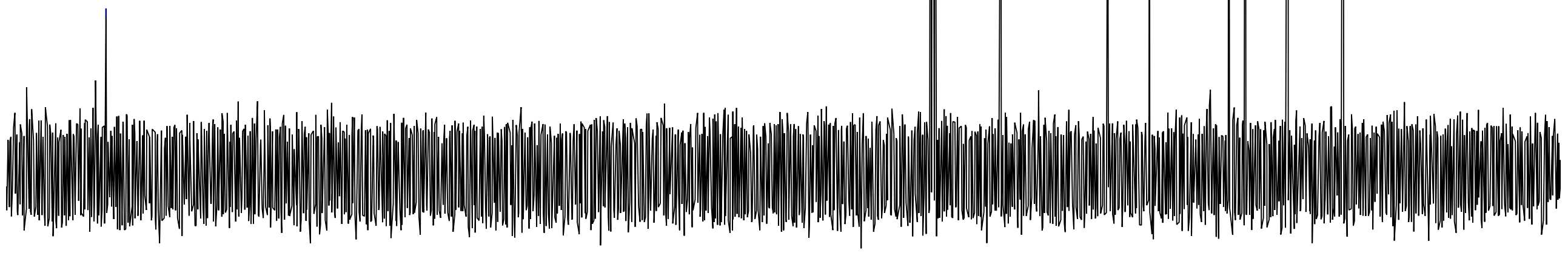


$400 \mathrm{MHz}, \mathrm{CDCl}_{3}$<smiles>CCCCCC(=S)N1CCCC1</smiles>

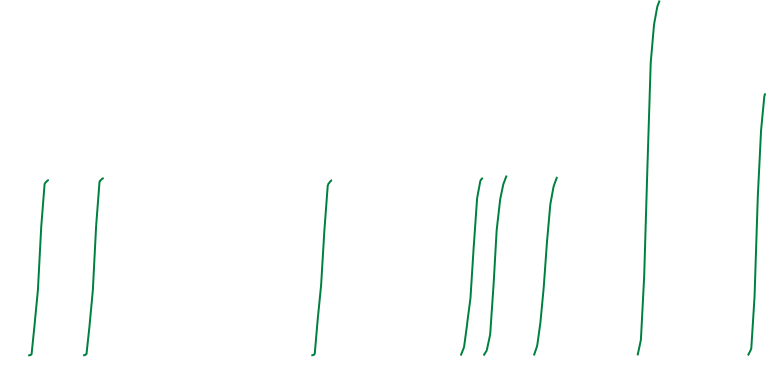

$5 m$

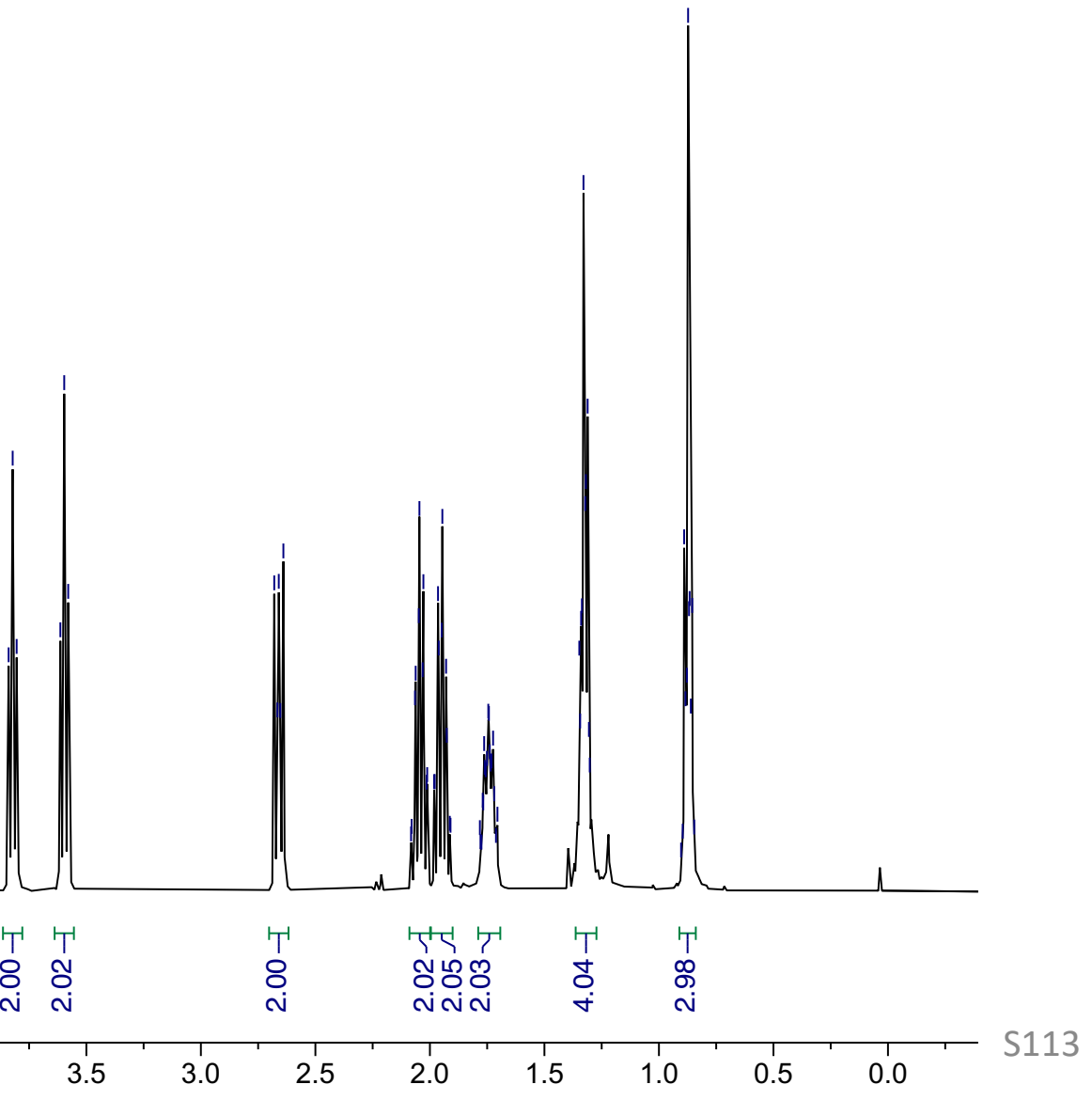


$101 \mathrm{MHz}, \mathrm{CDCl}_{3}$

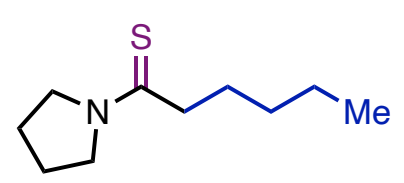

$5 \mathrm{~m}$

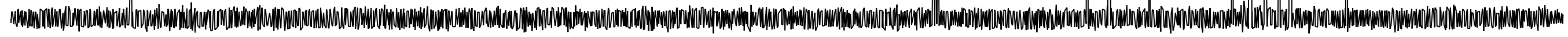


$400 \mathrm{MHz}, \mathrm{CDCl}_{3}$
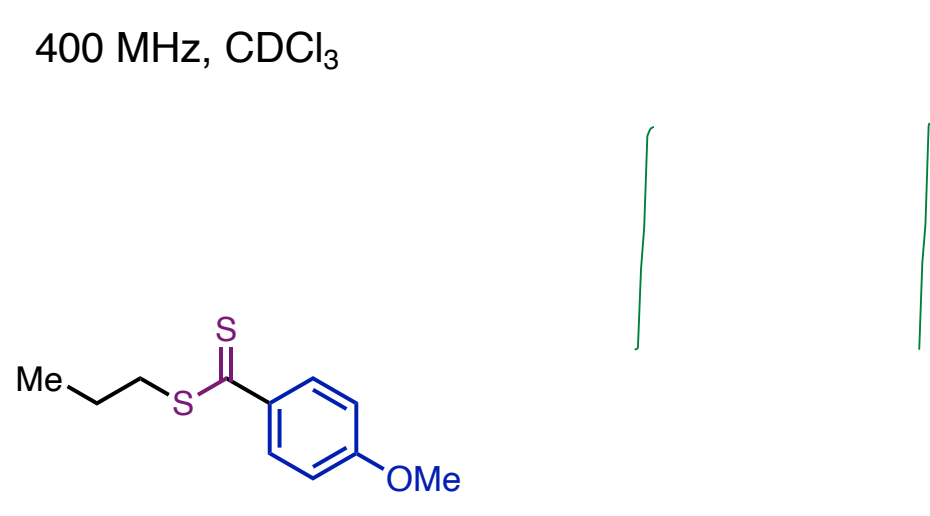

$6 a$

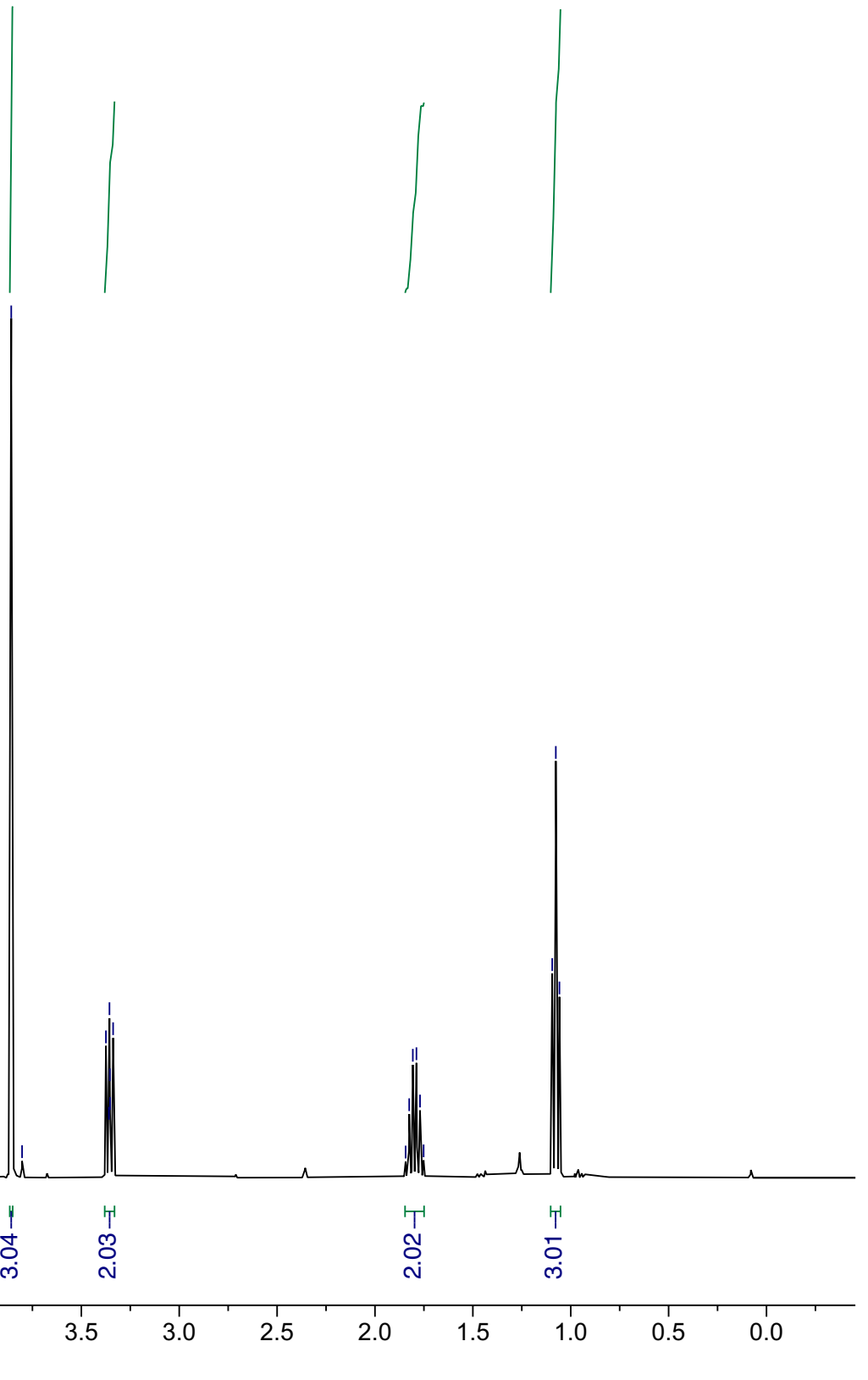




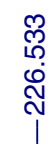

\begin{tabular}{l}
$\infty$ \\
0 \\
0 \\
0 \\
\hline
\end{tabular}

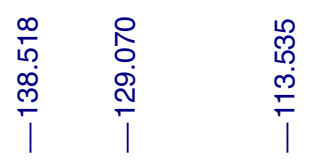

|

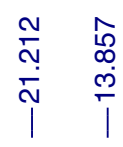

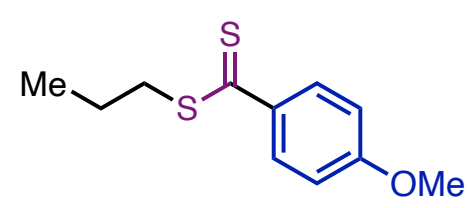

$6 a$

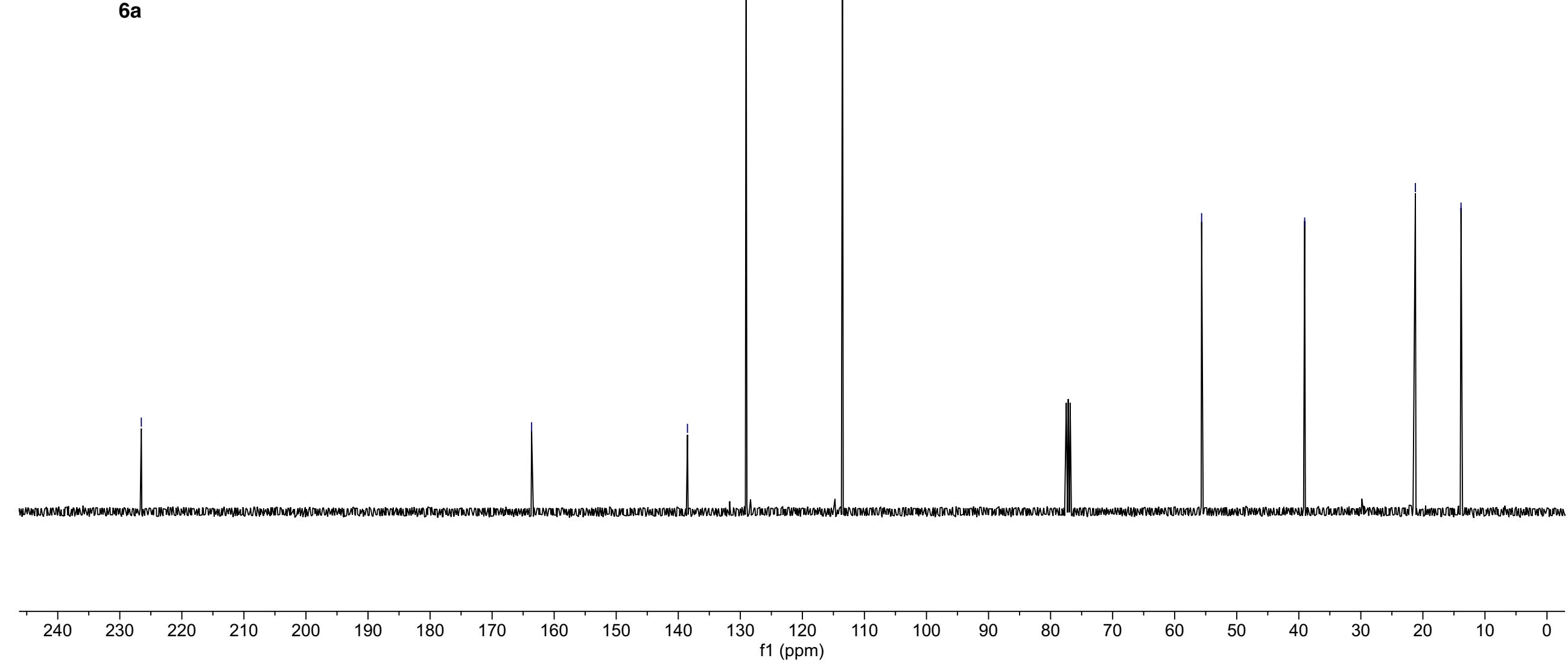

S116 
$400 \mathrm{MHz}, \mathrm{CDCl}_{3}$<smiles>CCCCCC1(OC(C)=S)CC1</smiles>

$1 a$
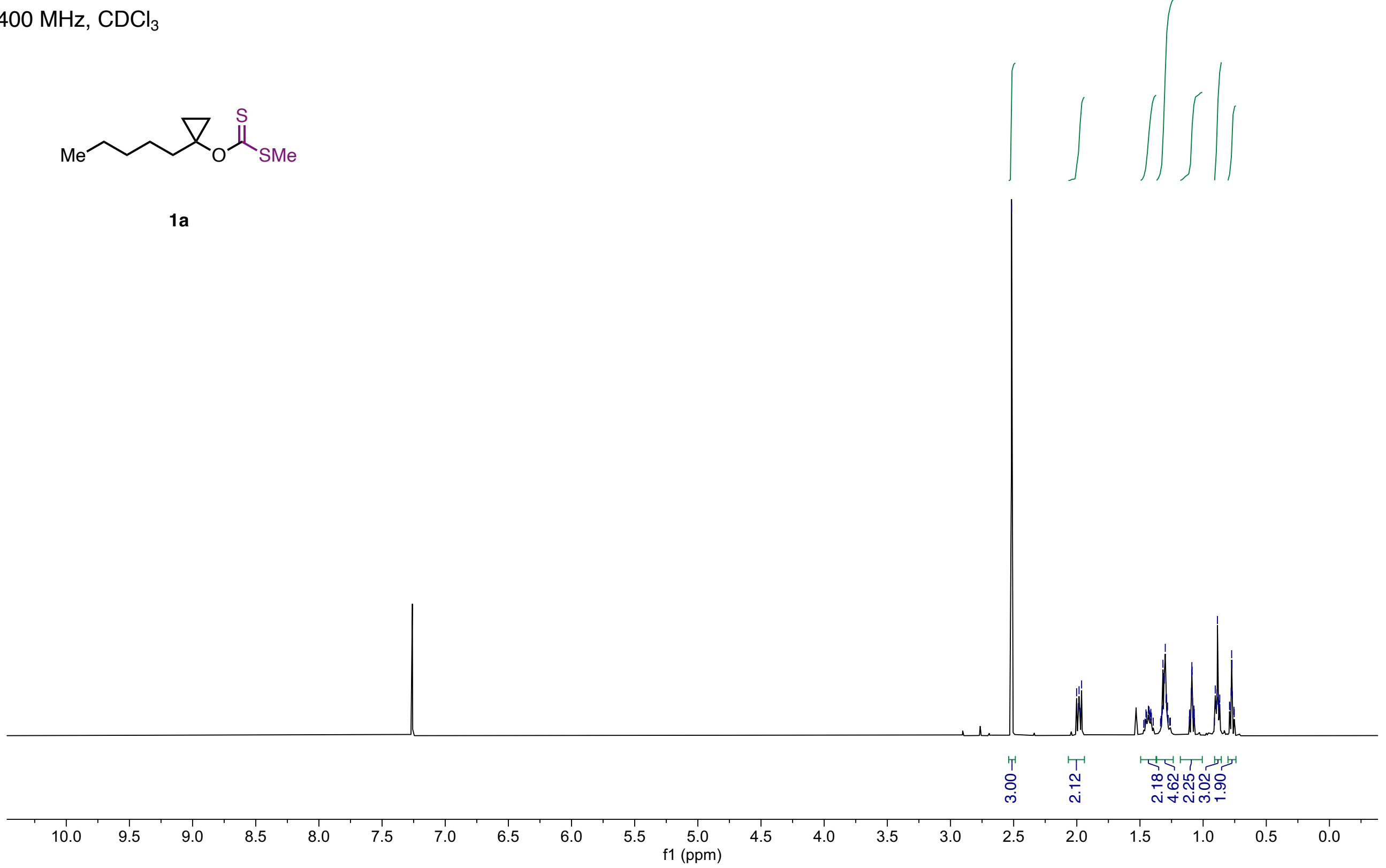


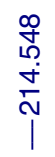

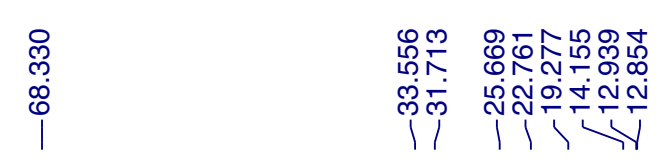

$101 \mathrm{MHz}, \mathrm{CDCl}_{3}$

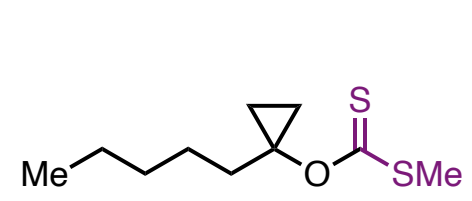

$1 a$

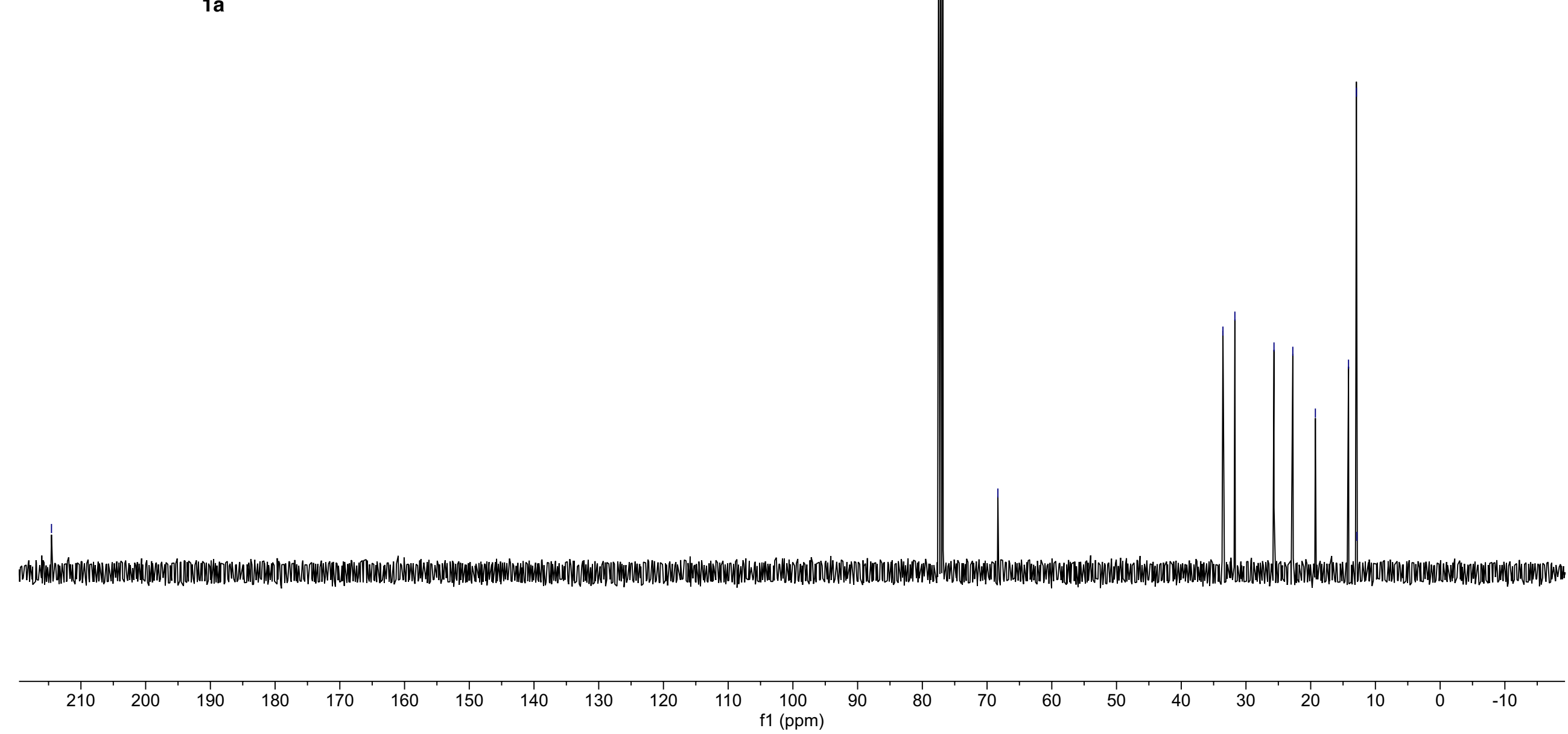

S118 
$400 \mathrm{MHz}, \mathrm{CDCl}_{3}$

$\nabla \cdot \cdot_{\mathrm{O}}^{\mathrm{Ph}} \stackrel{\mathrm{L}}{\mathrm{SMe}}_{\mathrm{SM}}$

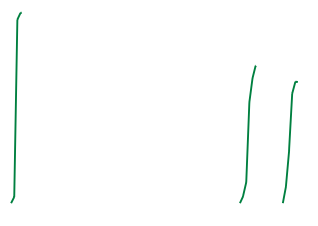

$1 b$
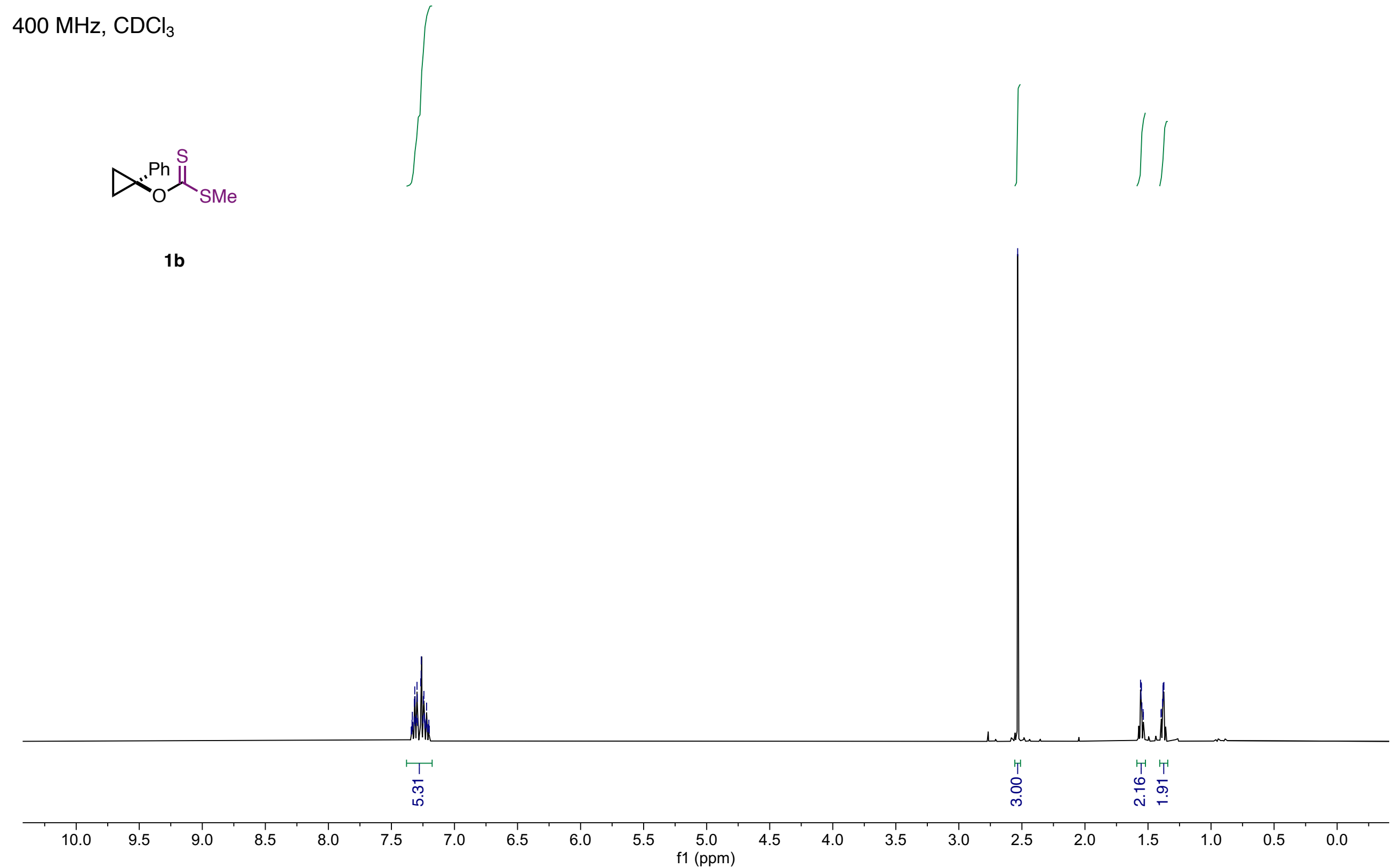


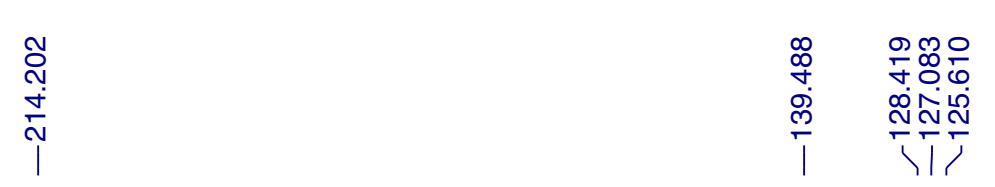

$100 \mathrm{MHz}, \mathrm{CDCl}_{3}$

$\nabla \cdot \stackrel{\mathrm{Ph}}{\mathrm{H}}_{\mathrm{SMe}}$

$1 b$

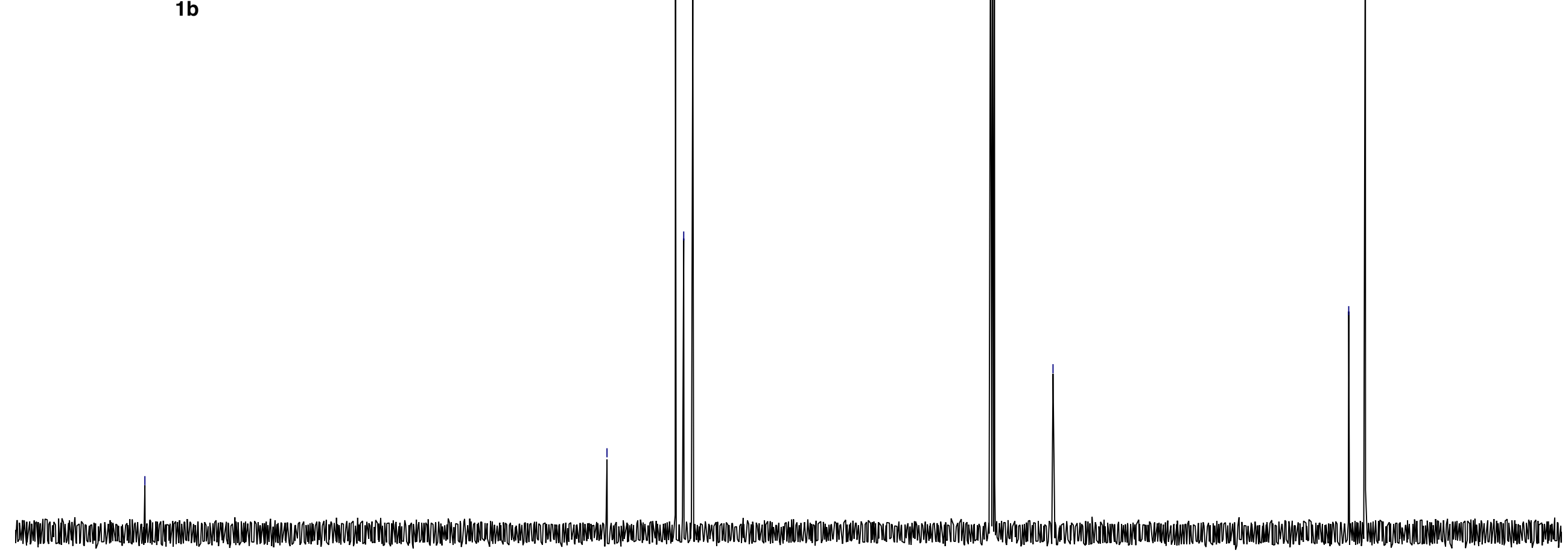


$500 \mathrm{MHz}, \mathrm{CDCl}_{3}$<smiles>CC(=S)OC(Cc1ccccc1)C(C)(C)C</smiles>
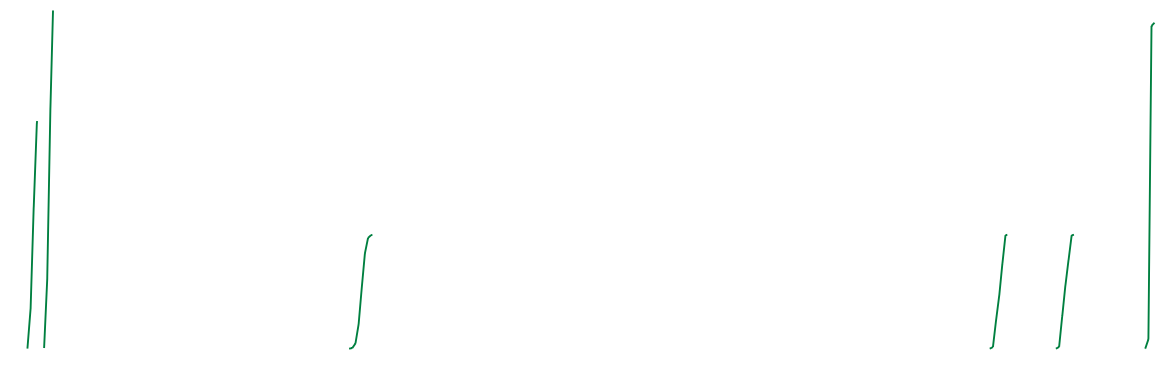

1c

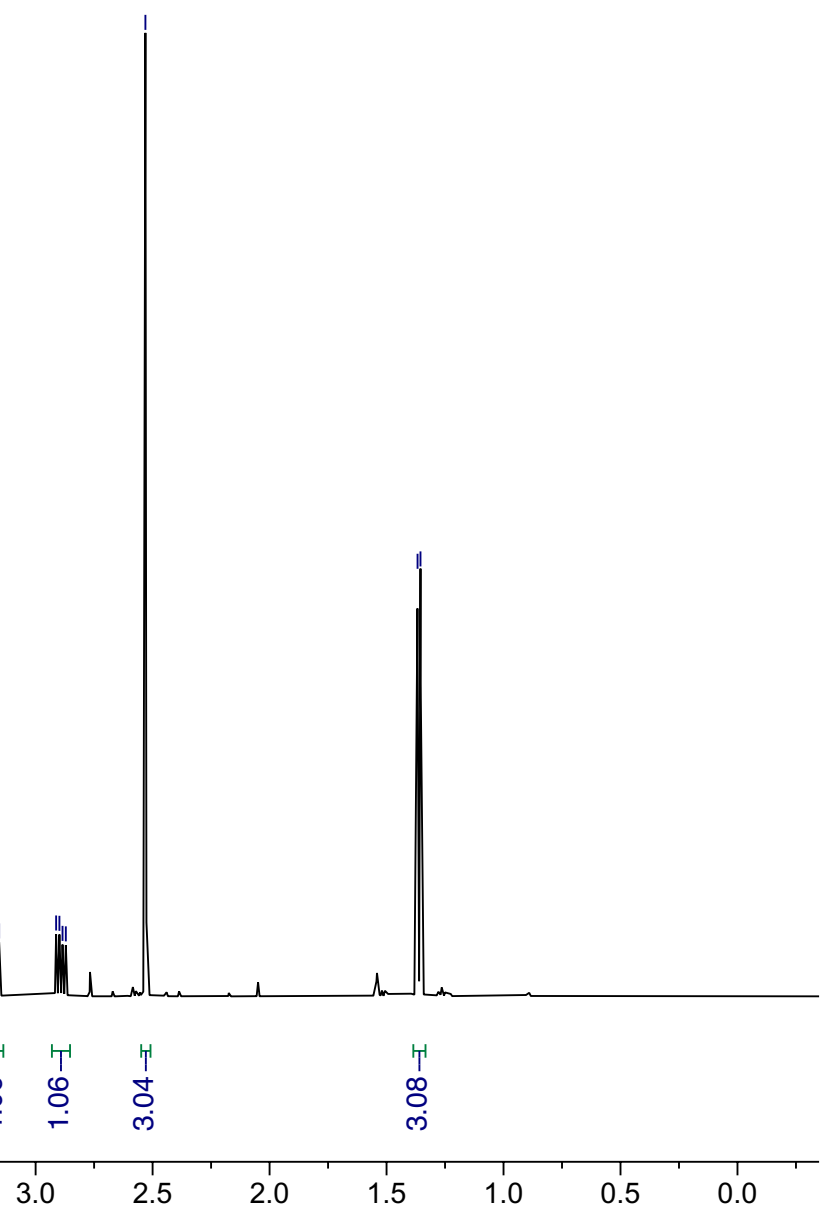




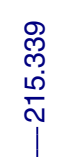

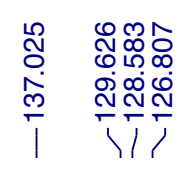

$\underset{\substack{\hat{n} \\ \infty}}{\substack{\infty \\ 1}}$

$\frac{i}{\frac{1}{+}}$

$\bar{\delta} \frac{N}{\infty}$
$\infty$
$\infty \frac{1}{\infty}$

$126 \mathrm{MHz} \mathrm{CDCl}_{3}$<smiles>CC(Cc1ccccc1)OC(=S)S(C)(=O)=O</smiles>

1c

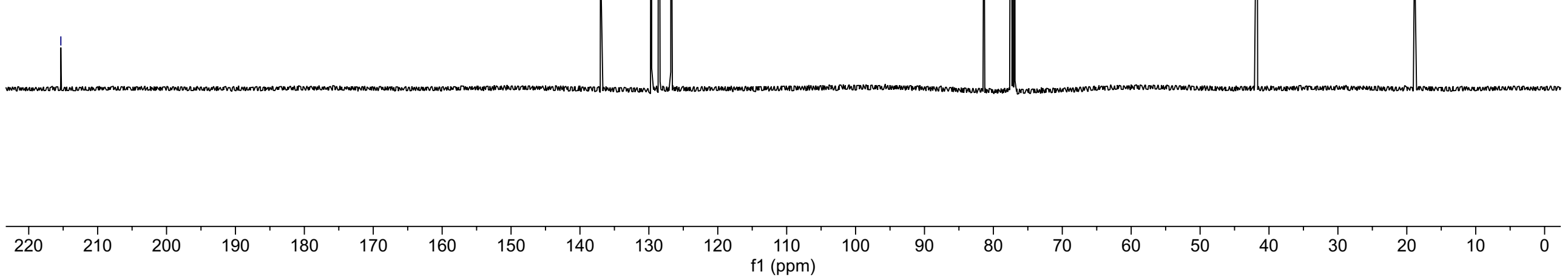

S122 
$400 \mathrm{MHz}, \mathrm{CDCl}_{3}$
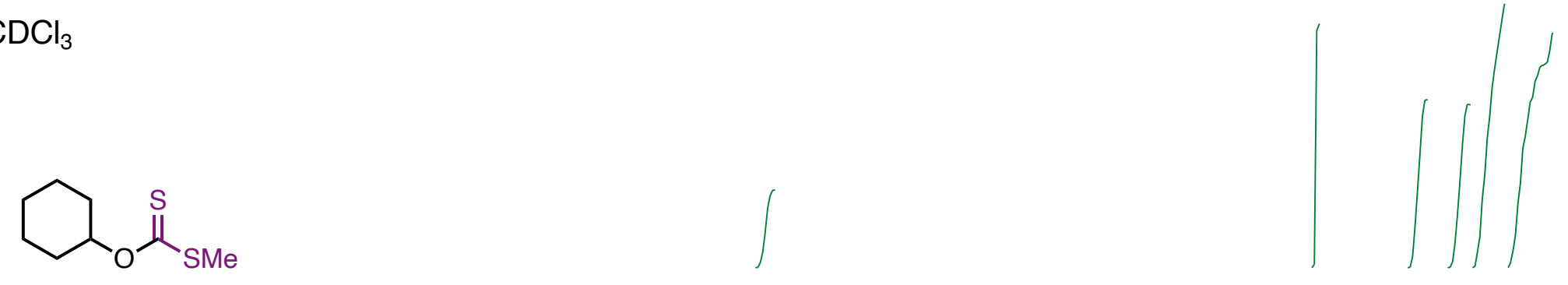

1d

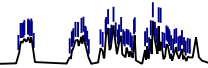

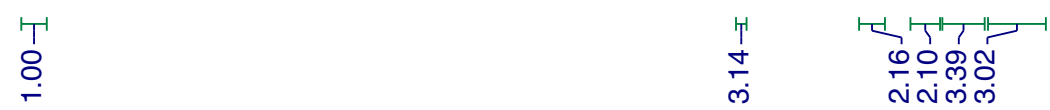

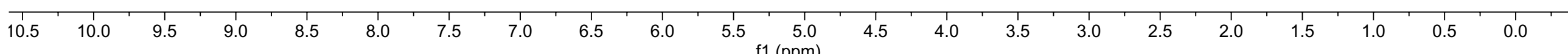


$100 \mathrm{MHz}, \mathrm{CDCl}_{3}$<smiles>CC(=S)OC1CCCCC1</smiles>

1d

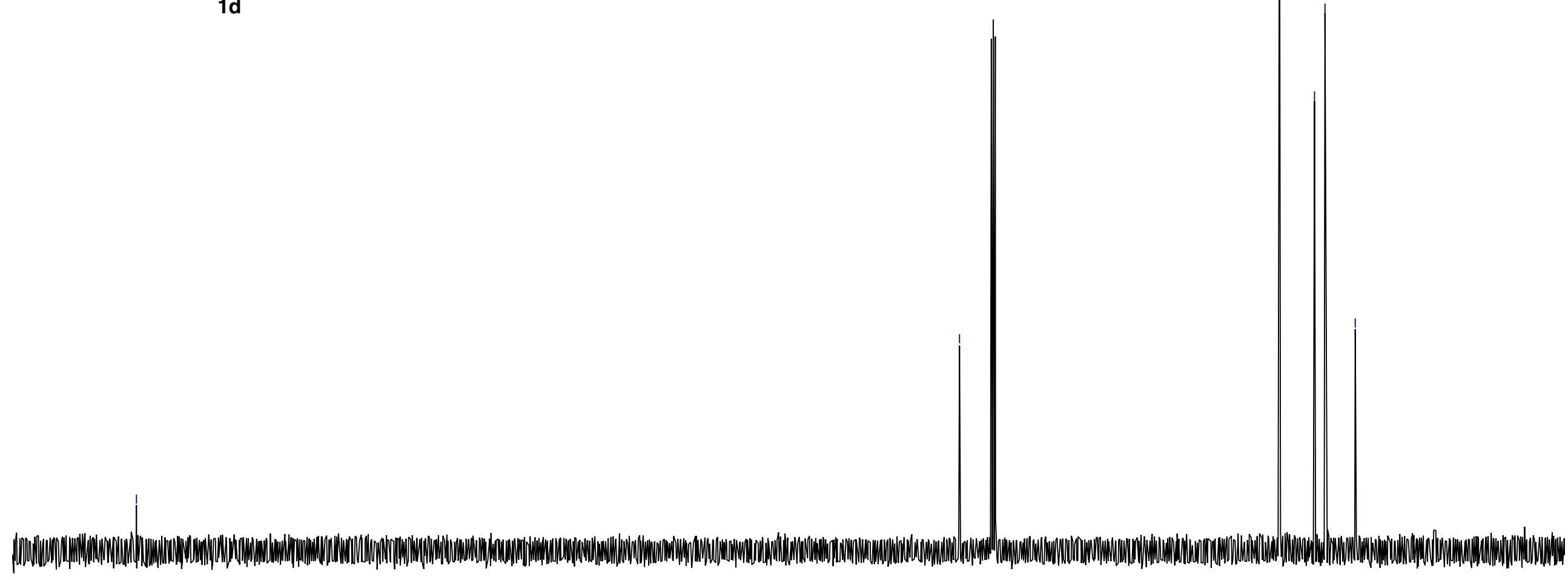


$500 \mathrm{MHz}, \mathrm{CDCl}_{3}$
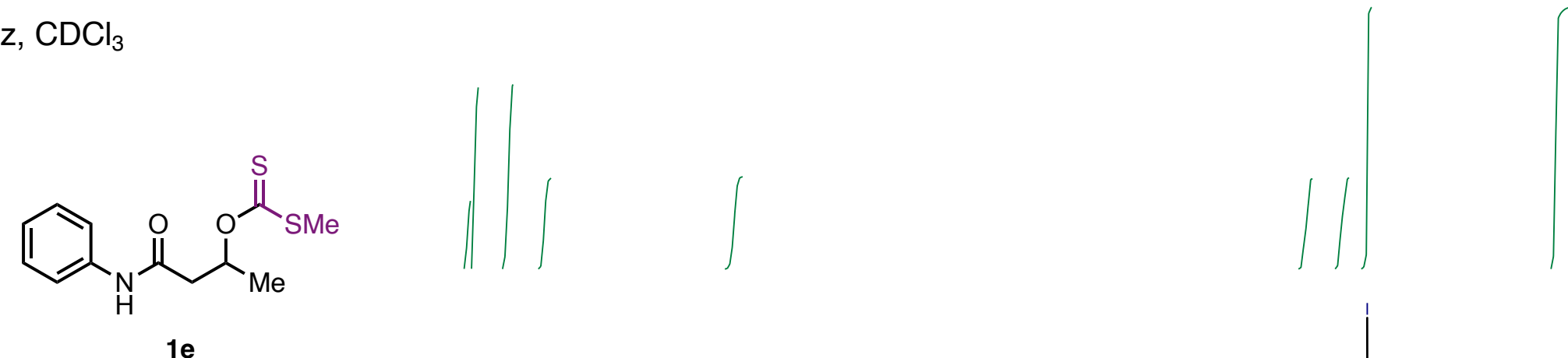

$1 e$

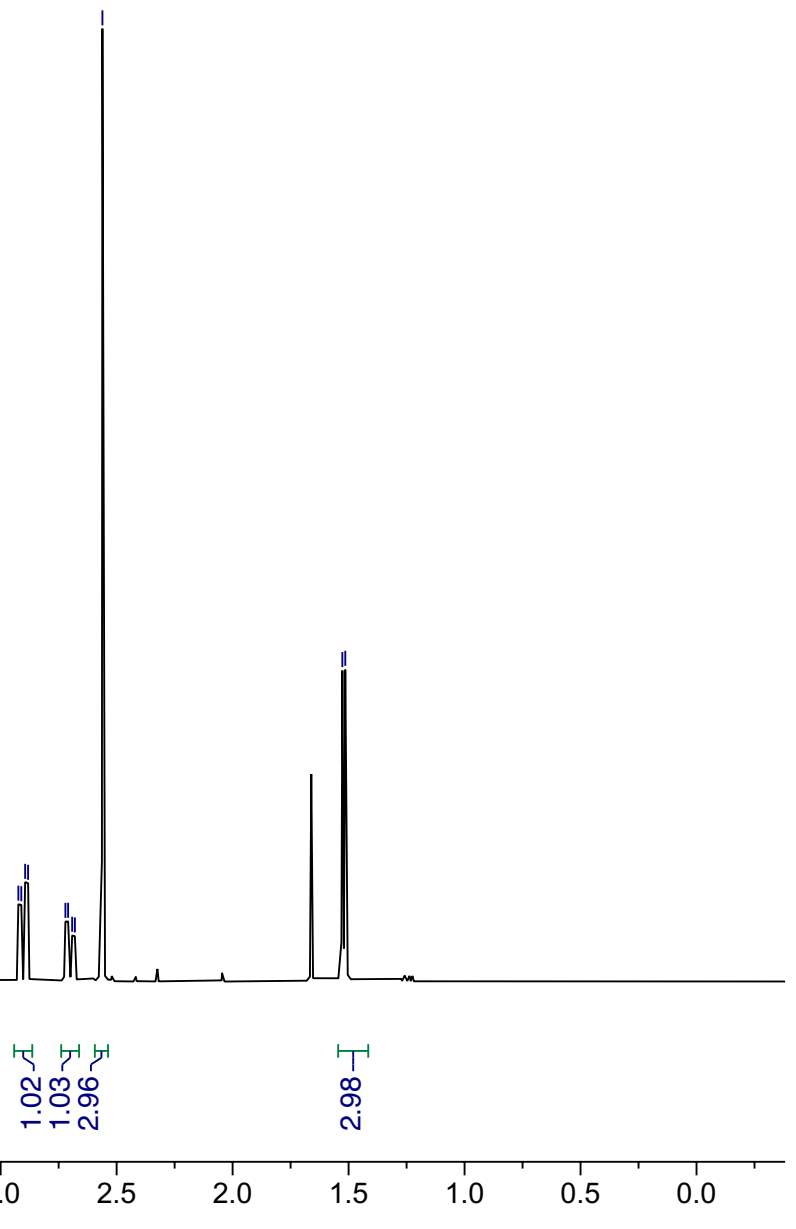




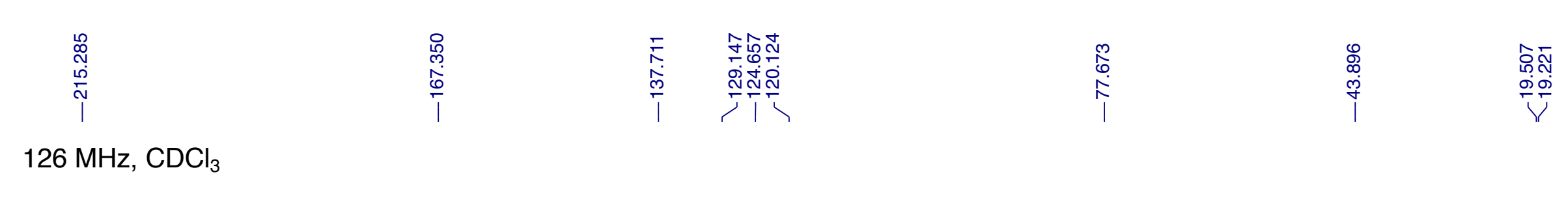

and

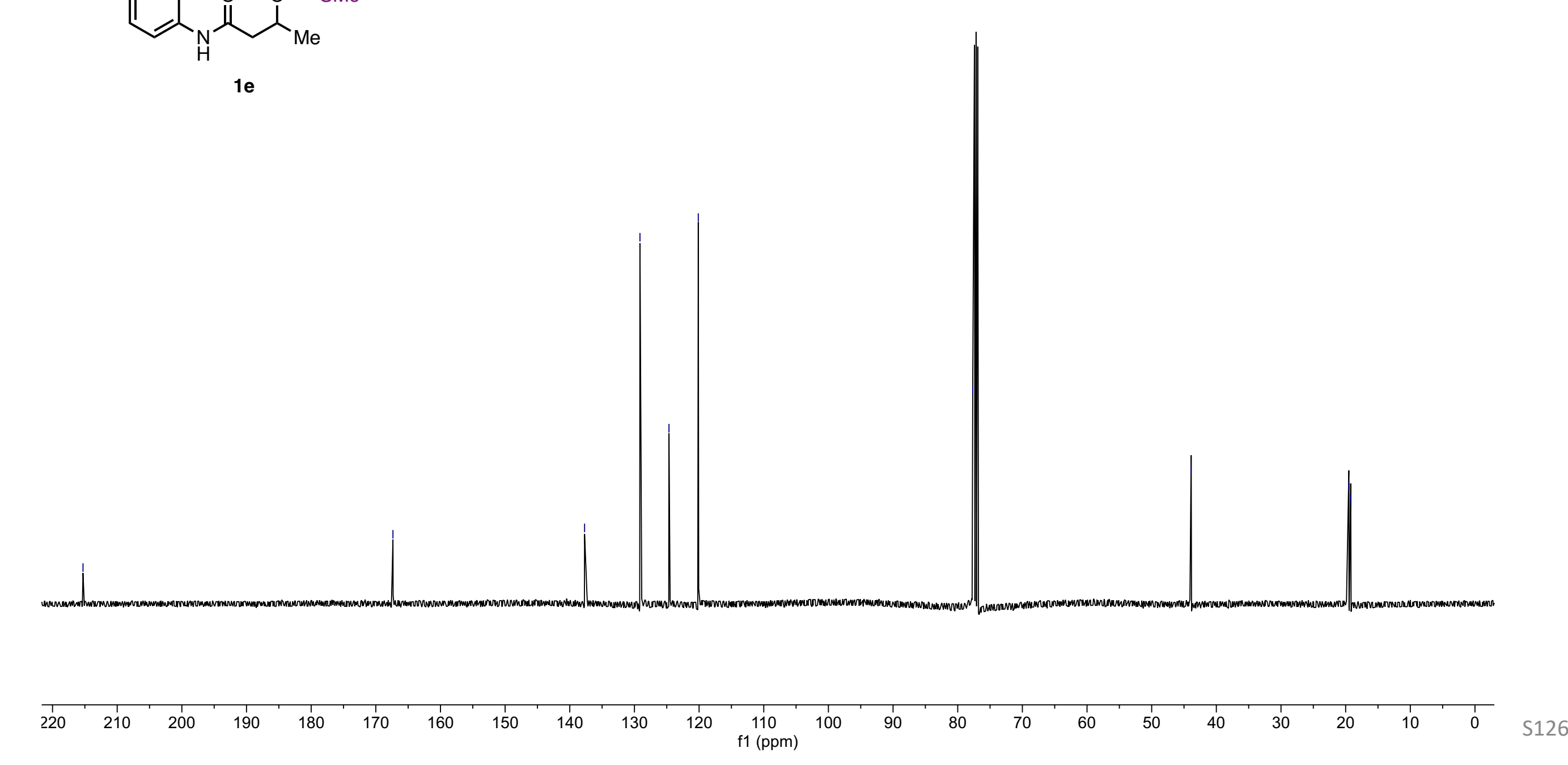


$400 \mathrm{MHz}, \mathrm{CDCl}_{3}$
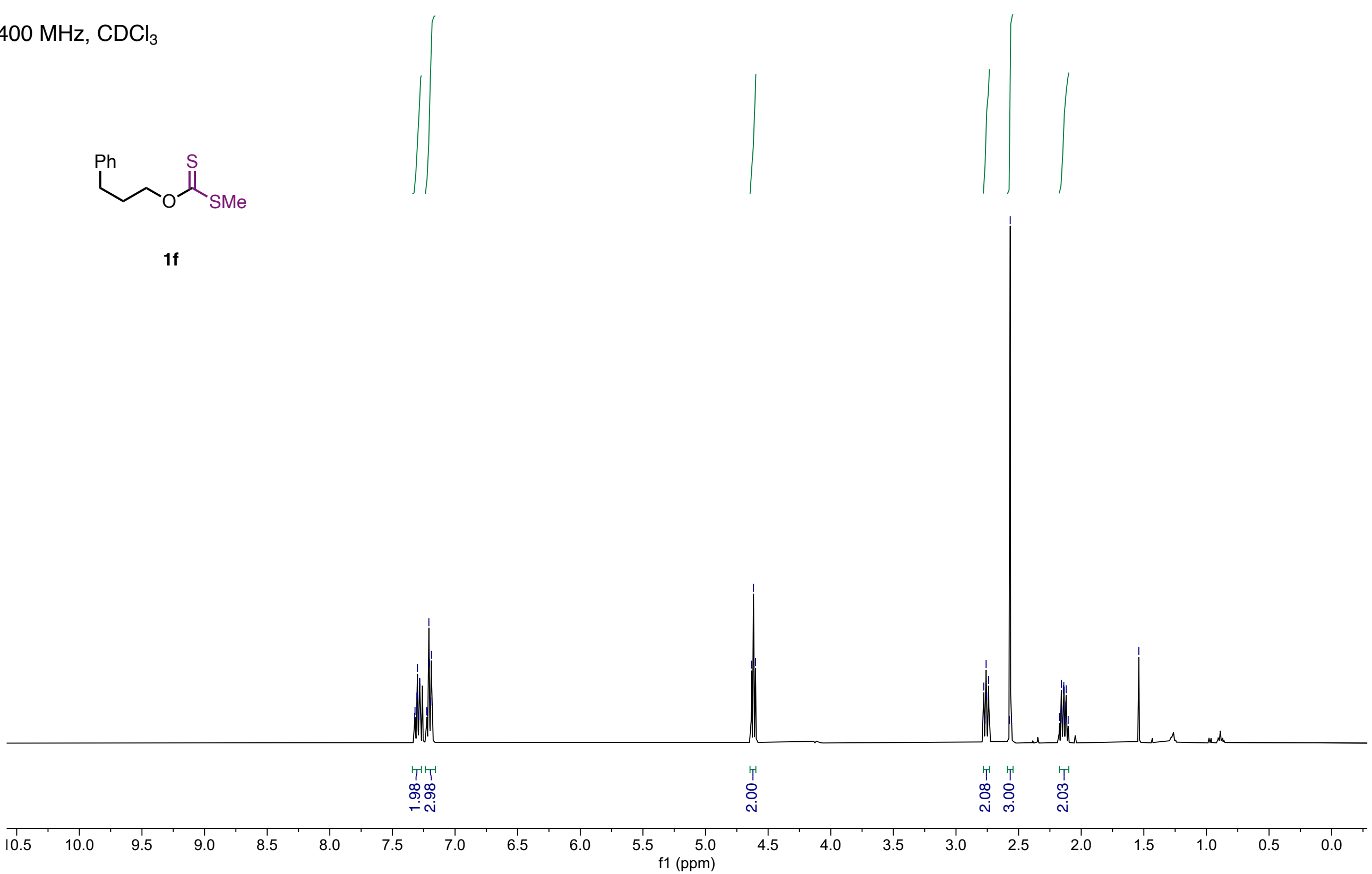
$\stackrel{+}{0}$
$\dot{0}$
$\stackrel{0}{N}$
1

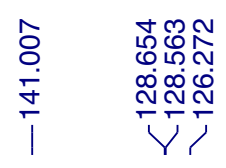

岂

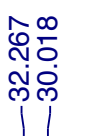

$\frac{\overline{0}}{\overline{0}}$

$101 \mathrm{MHz}, \mathrm{CDCl}_{3}$

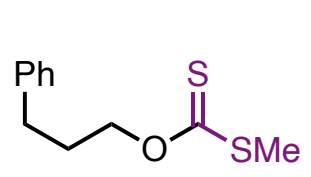

$1 f$

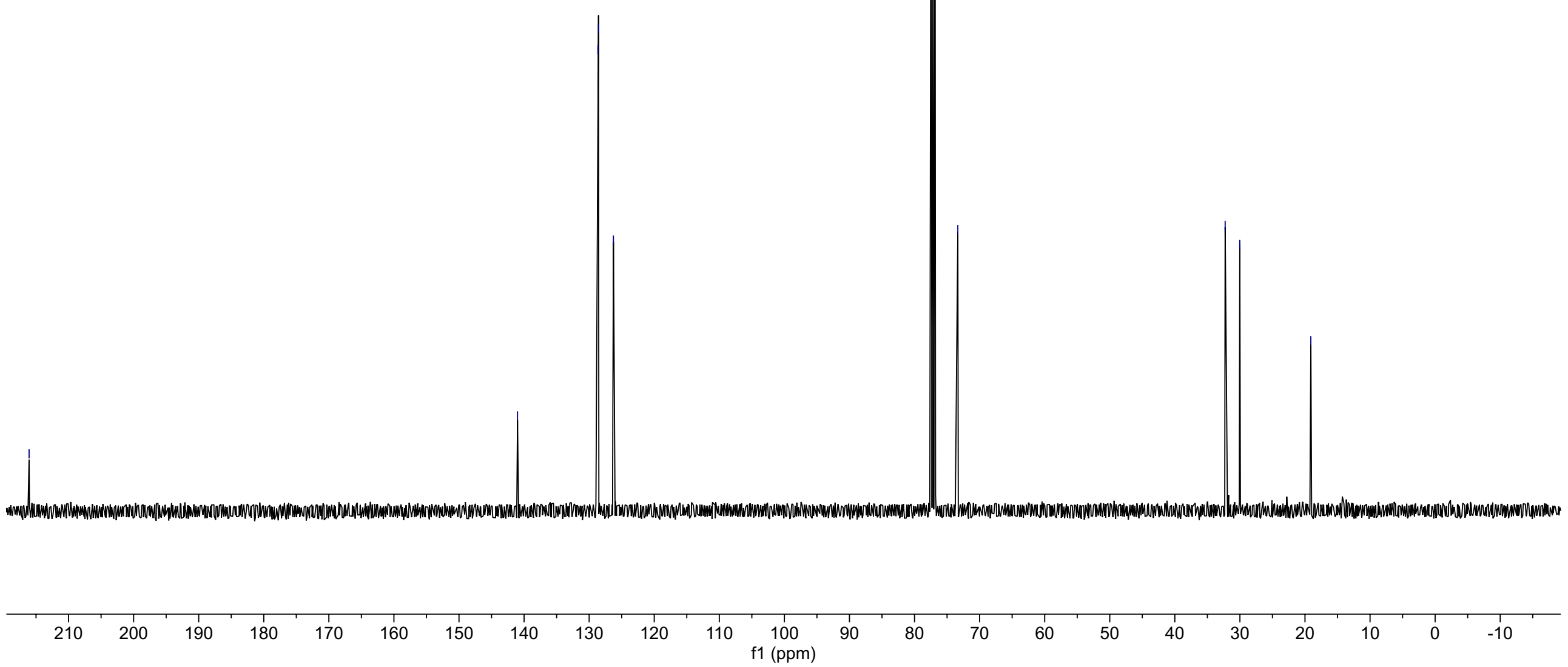

S128 
$500 \mathrm{MHz}, \mathrm{CDCl}_{3}$

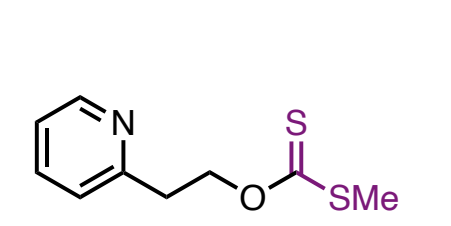

19

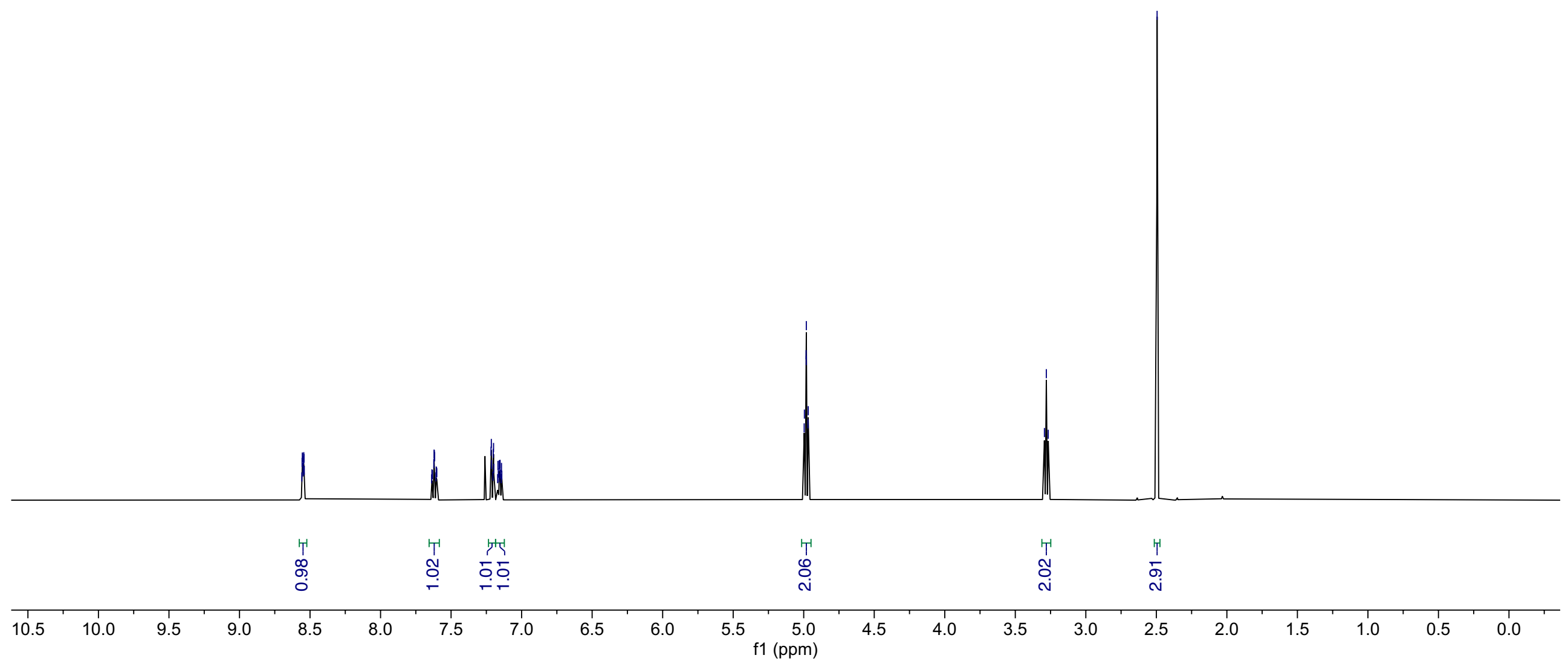




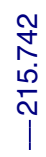

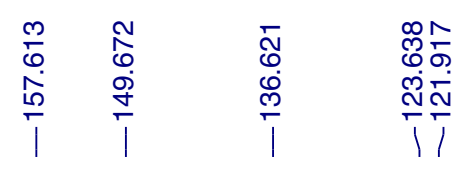

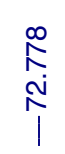

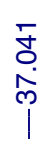

\begin{tabular}{l}
$\infty$ \\
$\substack{\$ \\
\infty \\
\infty}$ \\
\hline
\end{tabular}

$126 \mathrm{MHz}^{\mathrm{CDCl}_{3}}$<smiles>CSC(=S)OCCc1ccccn1</smiles>

19

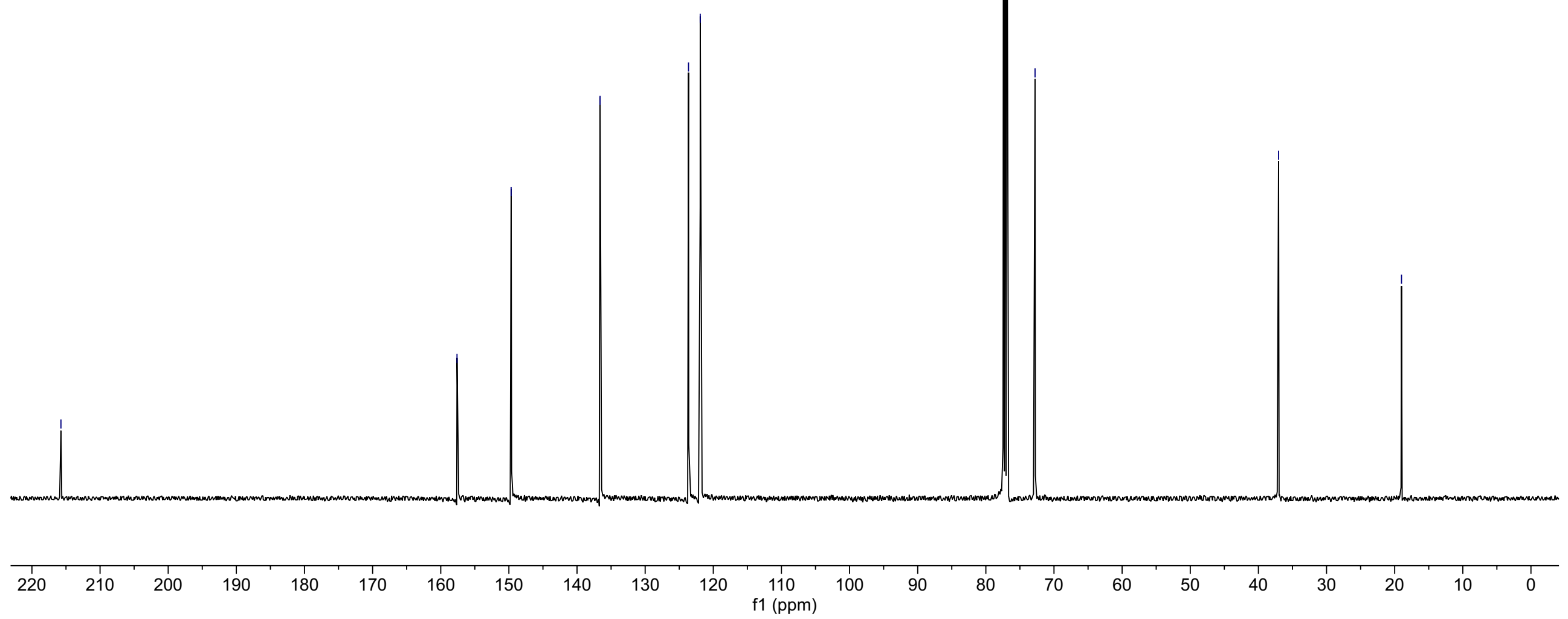

S130 
$400 \mathrm{MHz}, \mathrm{CDCl}_{3}$

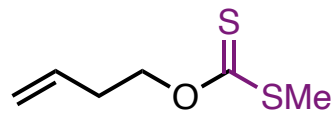

1h

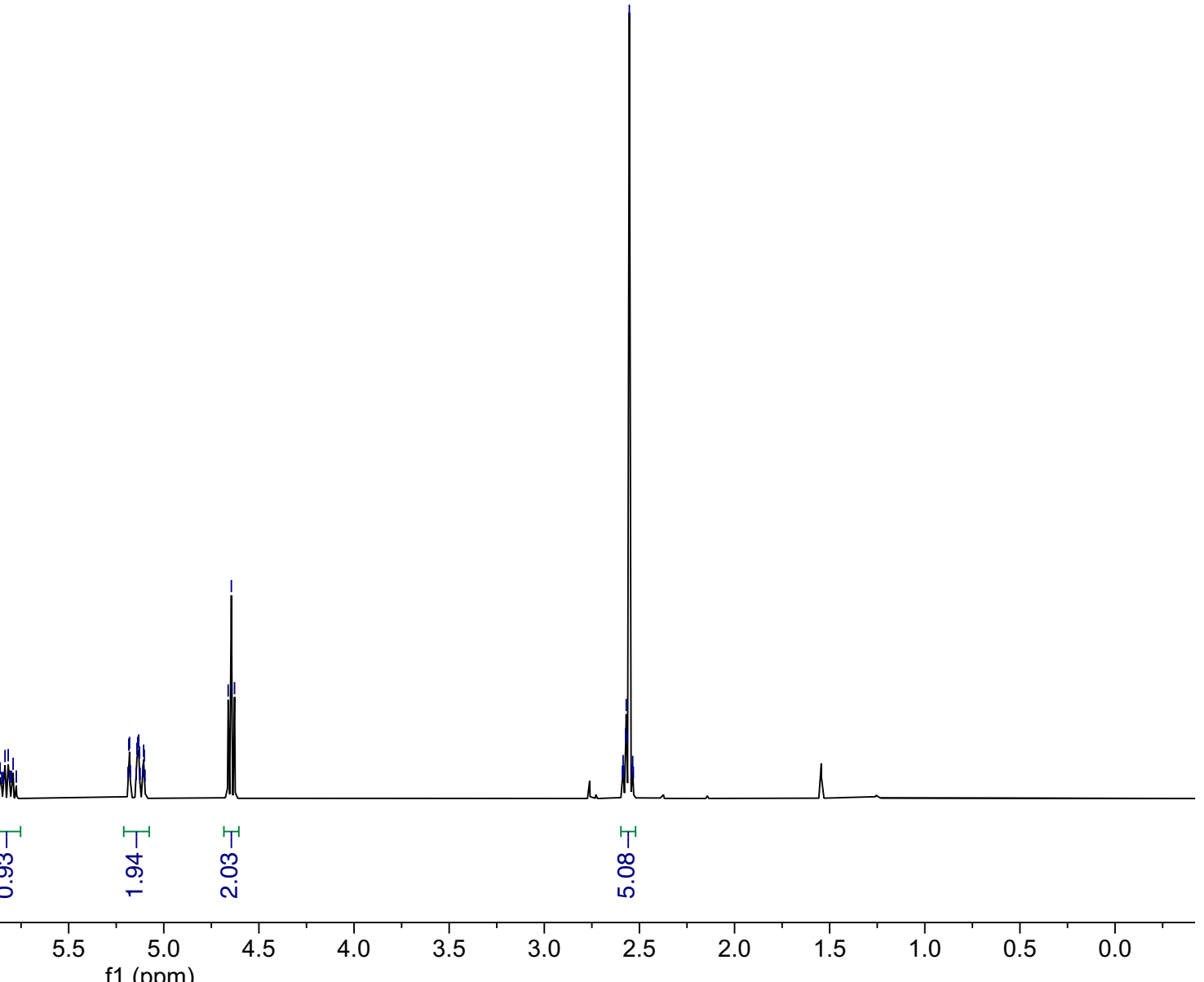




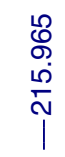



$\infty$
$\infty$
$\infty$
$\mathfrak{N}$
N

$\begin{array}{ll}\stackrel{2}{N} & \bar{\sigma} \\ \stackrel{0}{N} & \frac{0}{1}\end{array}$

$101 \mathrm{MHz}, \mathrm{CDCl}_{3}$

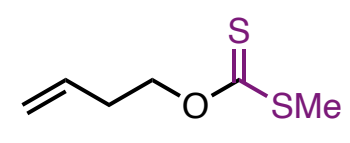

1h

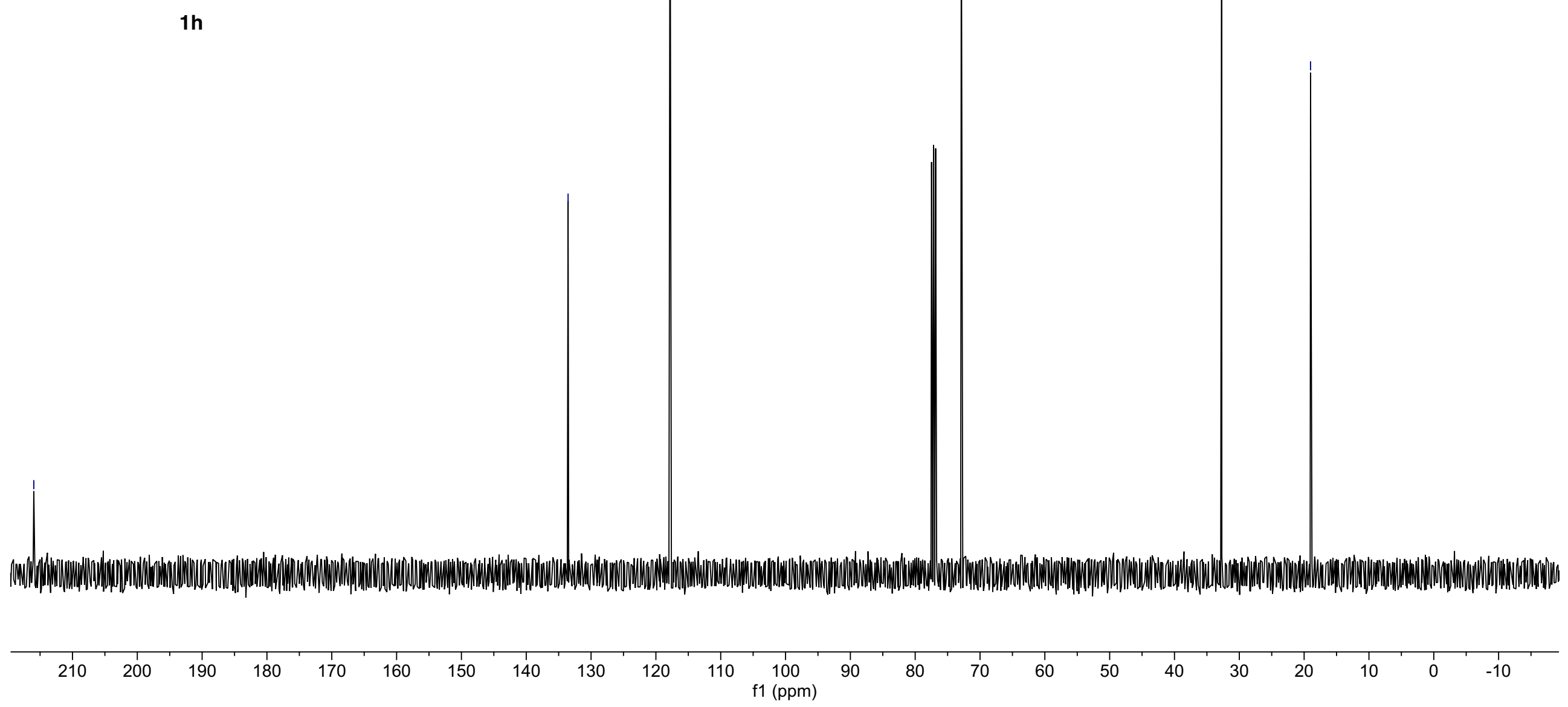

S132 
$500 \mathrm{MHz}, \mathrm{CDCl}_{3}$<smiles>C#CCCOC(=S)OC</smiles>

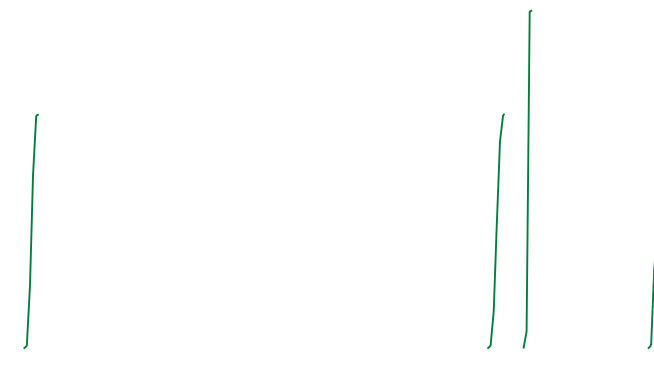

$1 i$

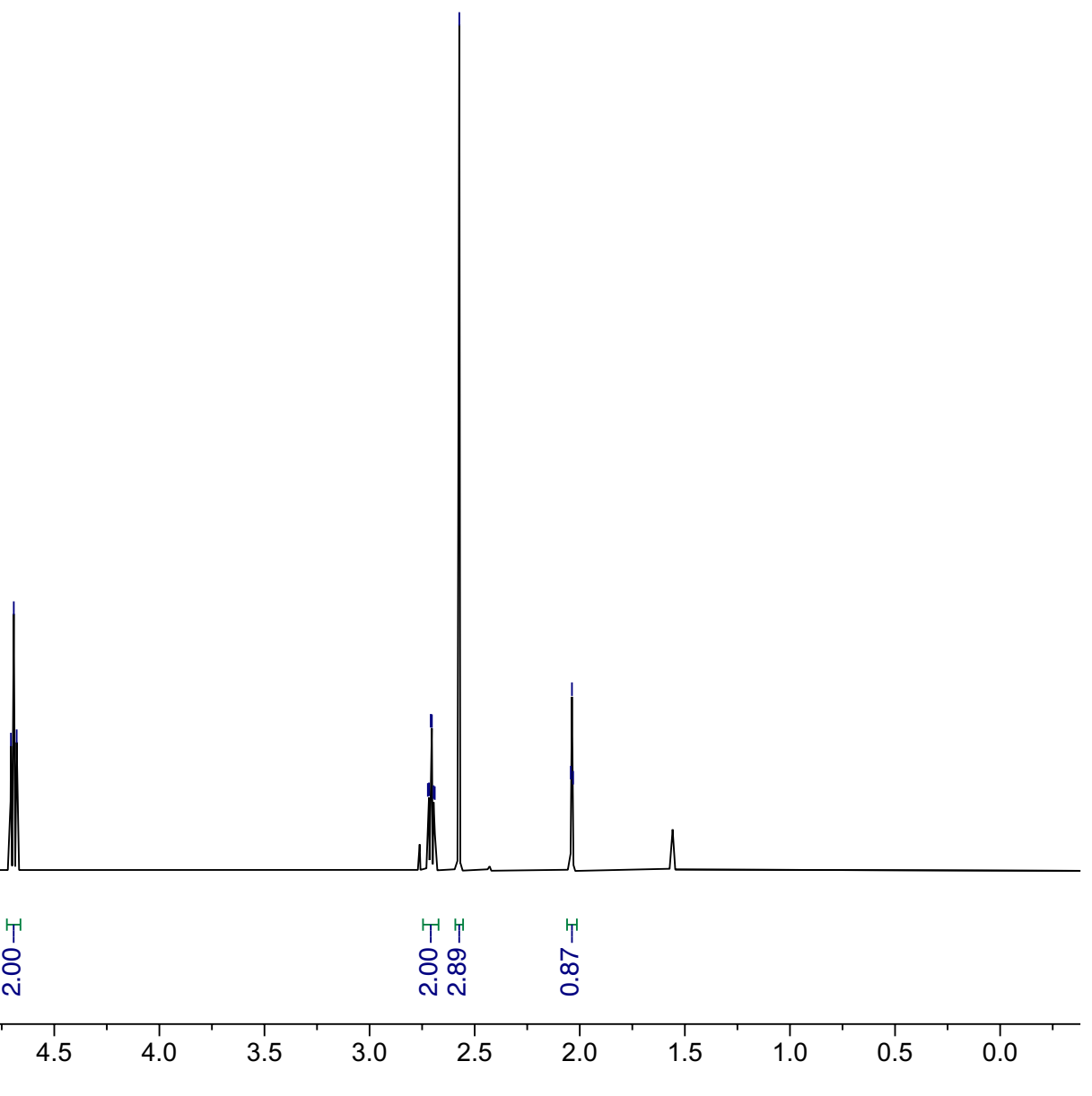


$126 \mathrm{MHz}, \mathrm{CDCl}_{3}$

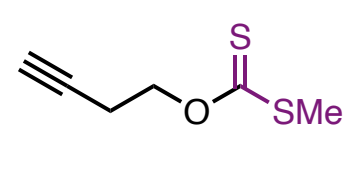

$1 \mathrm{i}$ 
$500 \mathrm{MHz}, \mathrm{CDCl}_{3}$

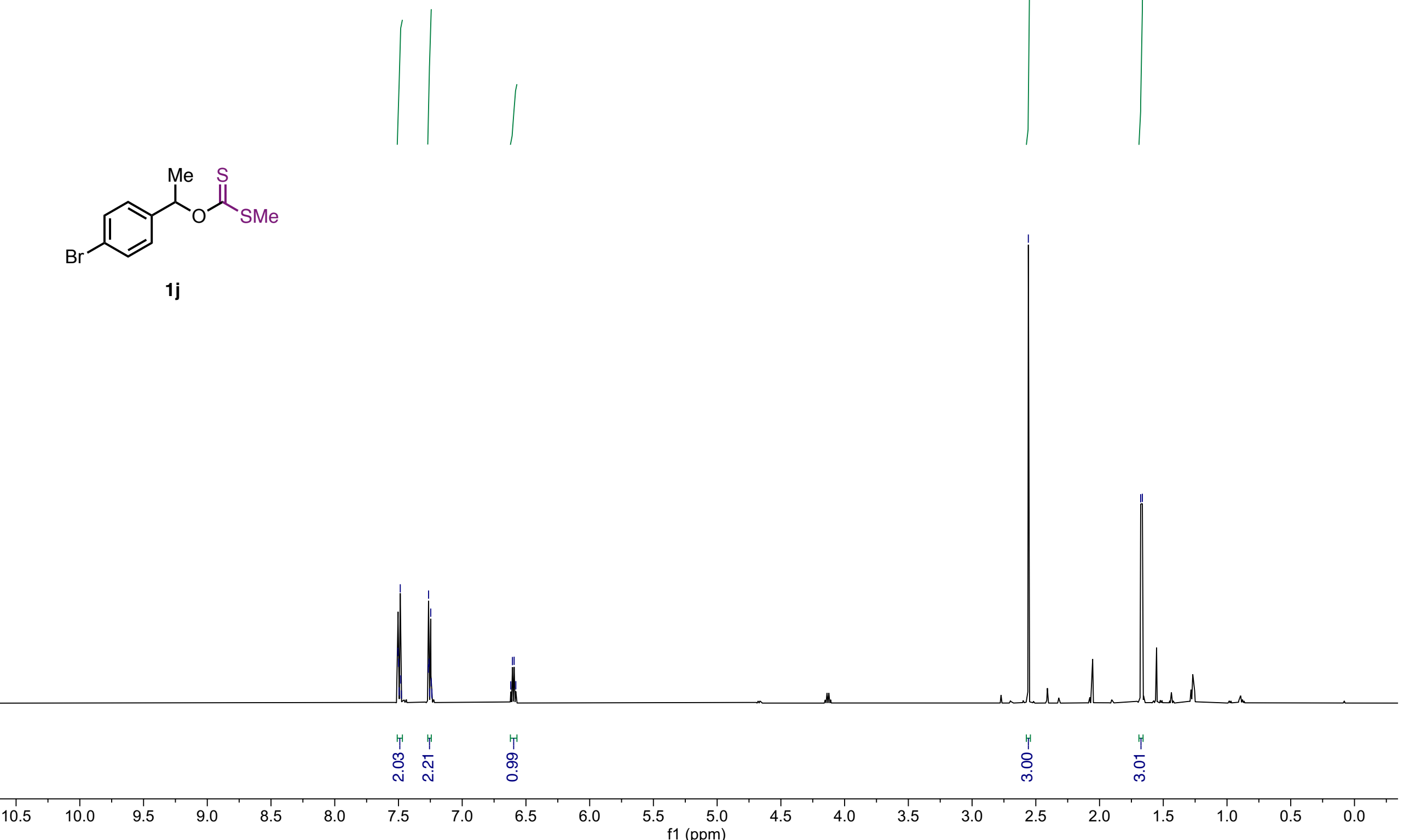




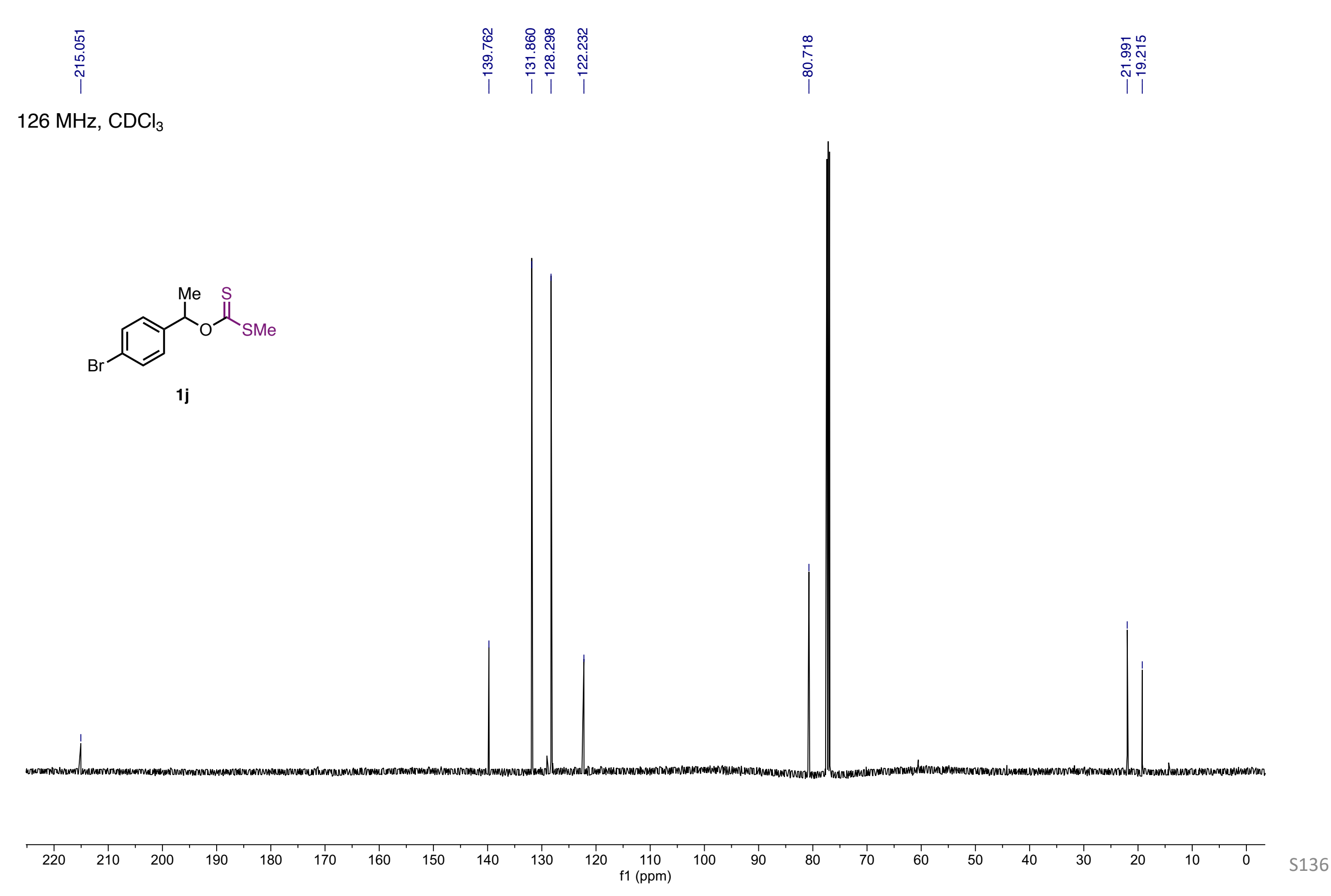


$400 \mathrm{MHz}, \mathrm{CDCl}_{3}$<smiles>[M]C(OC(=S)OC)c1ccccc1</smiles>

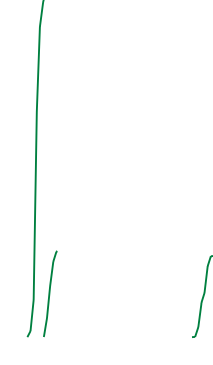

$1 \mathrm{k}$

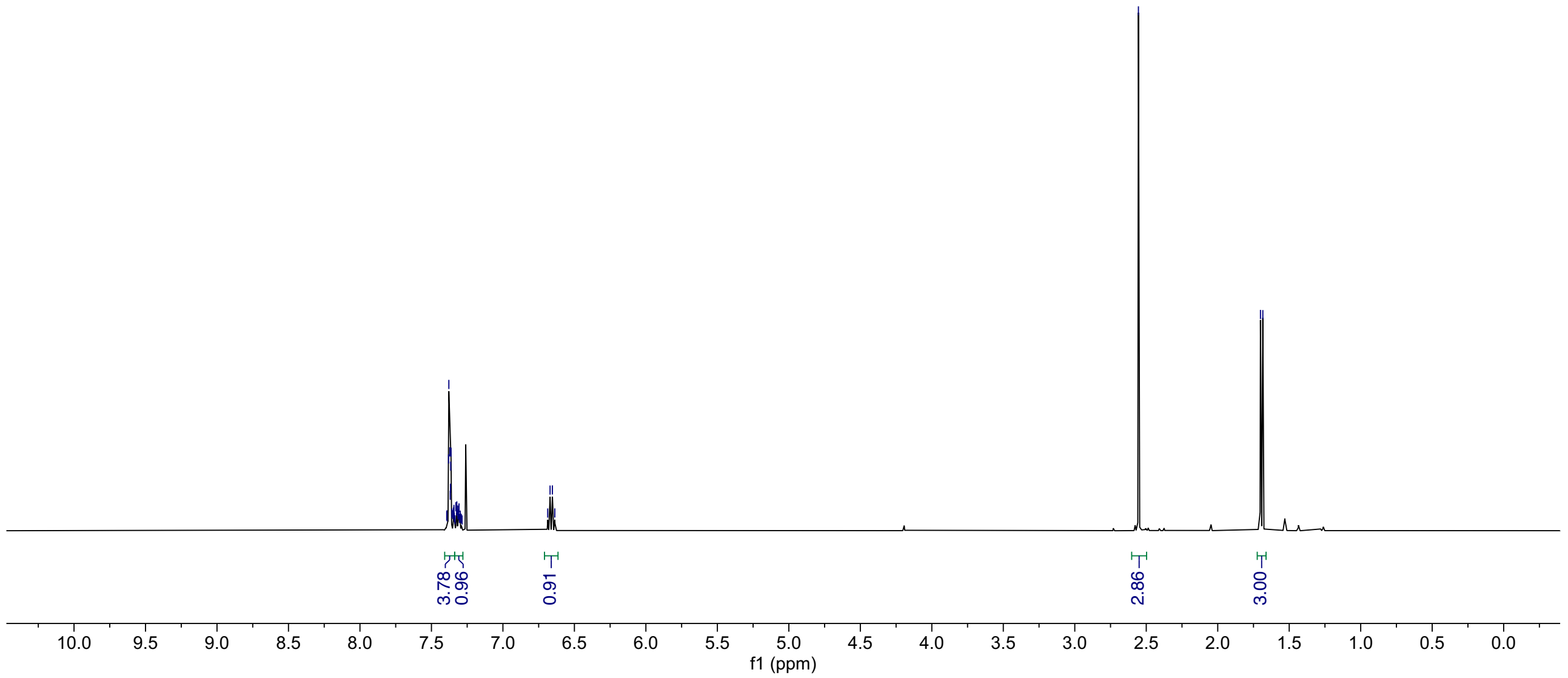




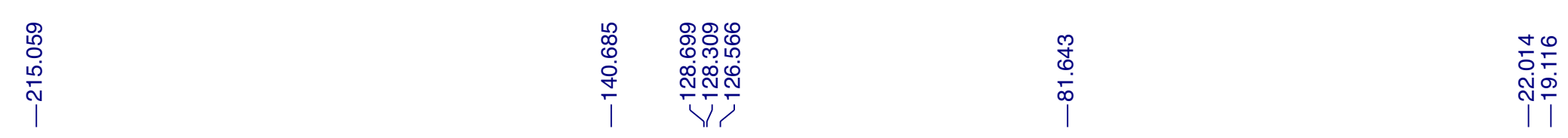

$100 \mathrm{MHz}, \mathrm{CDCl}_{3}$

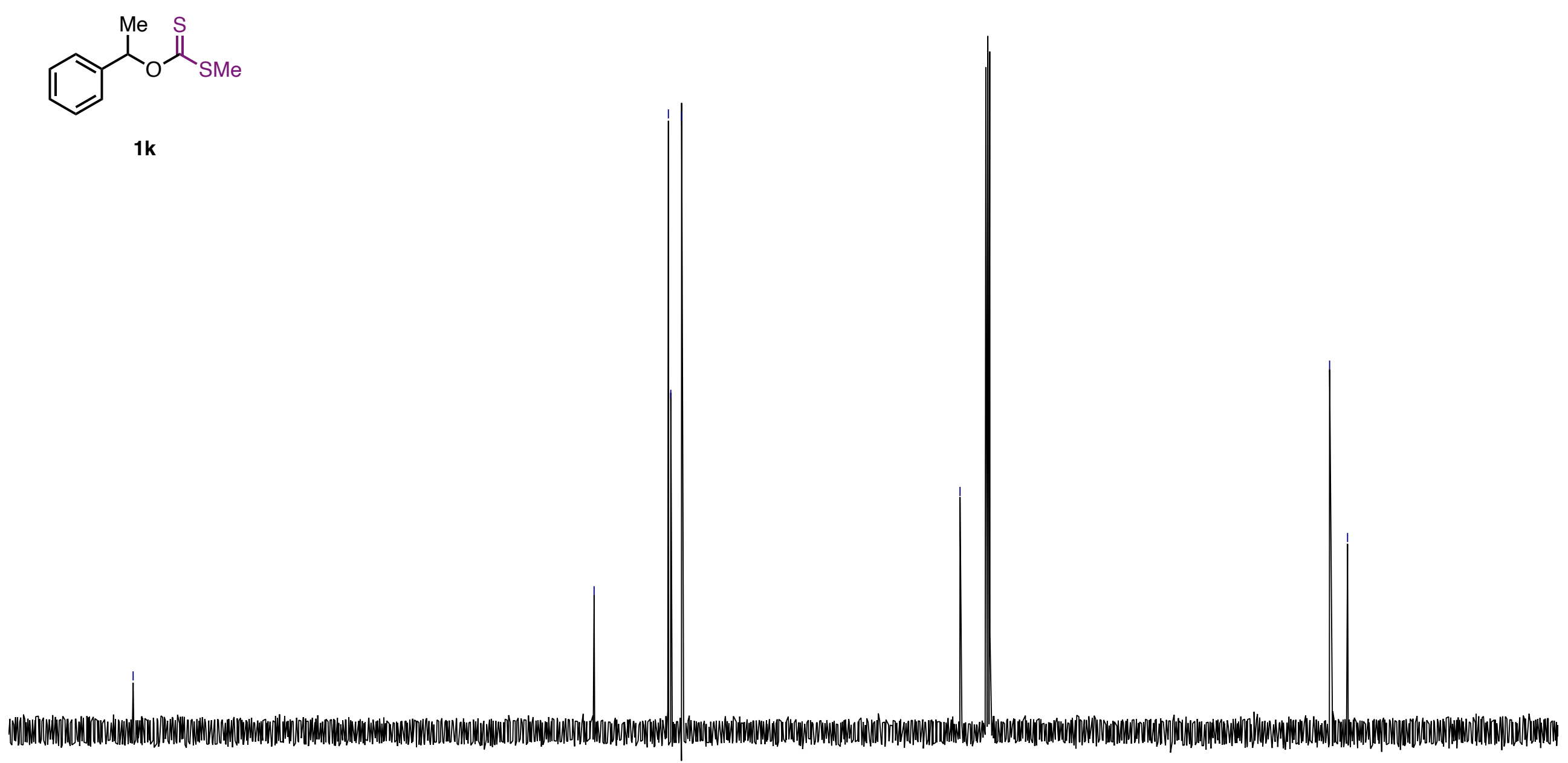


$500 \mathrm{MHz}, \mathrm{CDCl}_{3}$

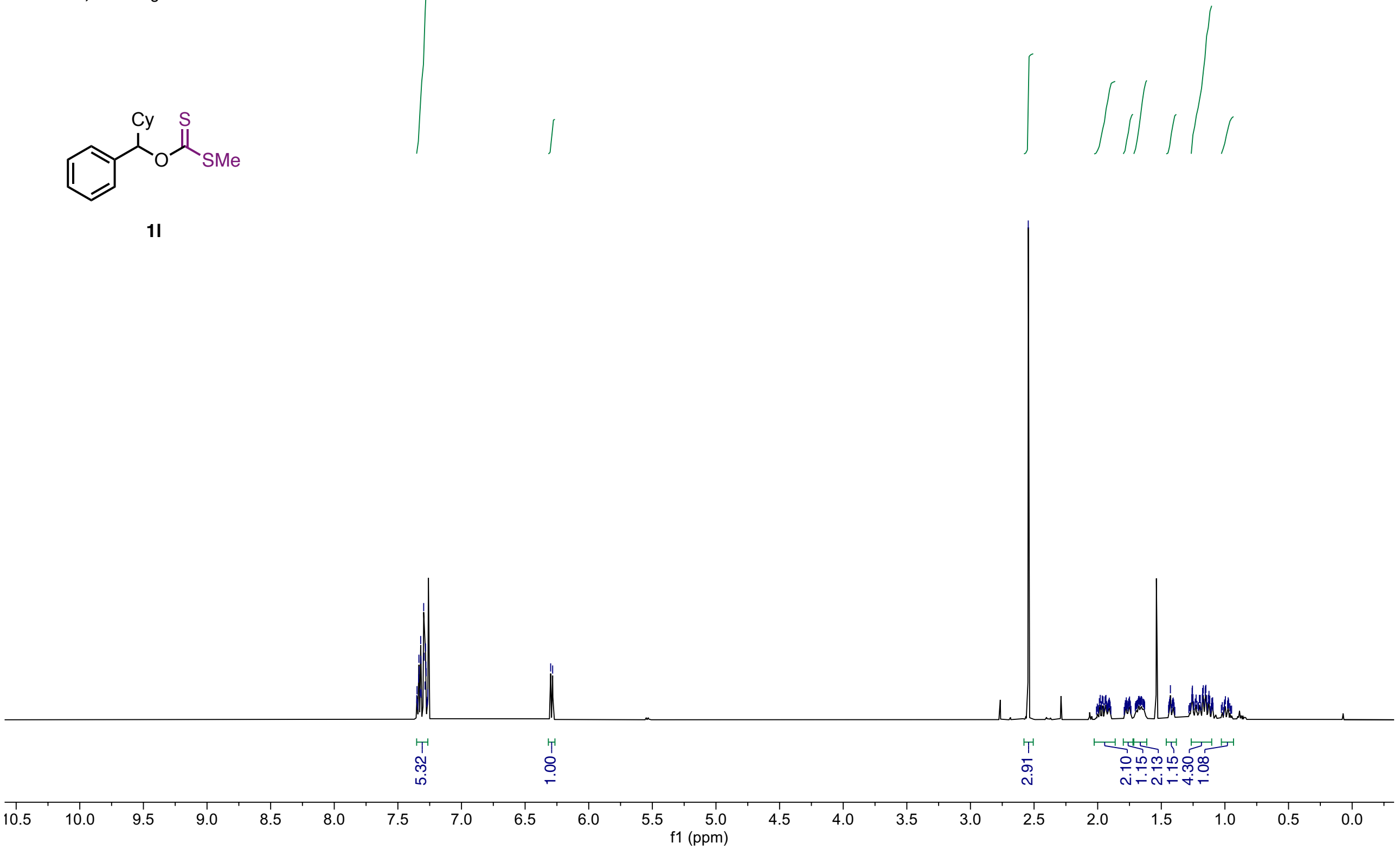


$126 \mathrm{MHz}, \mathrm{CDCl}_{3}$

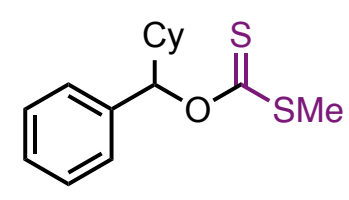

11

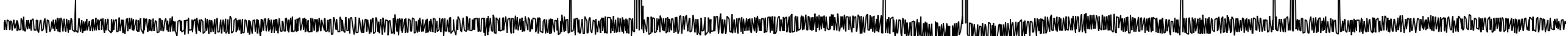

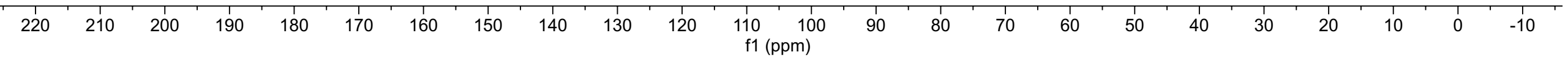


$500 \mathrm{MHz}, \mathrm{CDCl}_{3}$
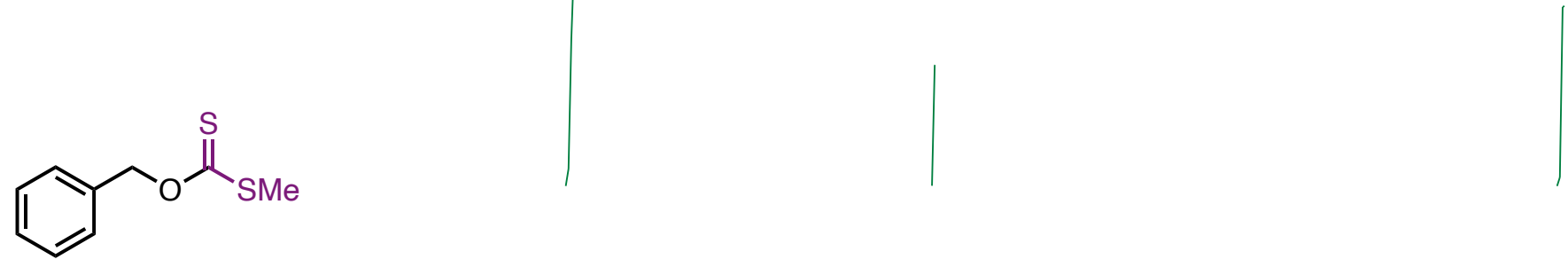

$1 \mathrm{~m}$

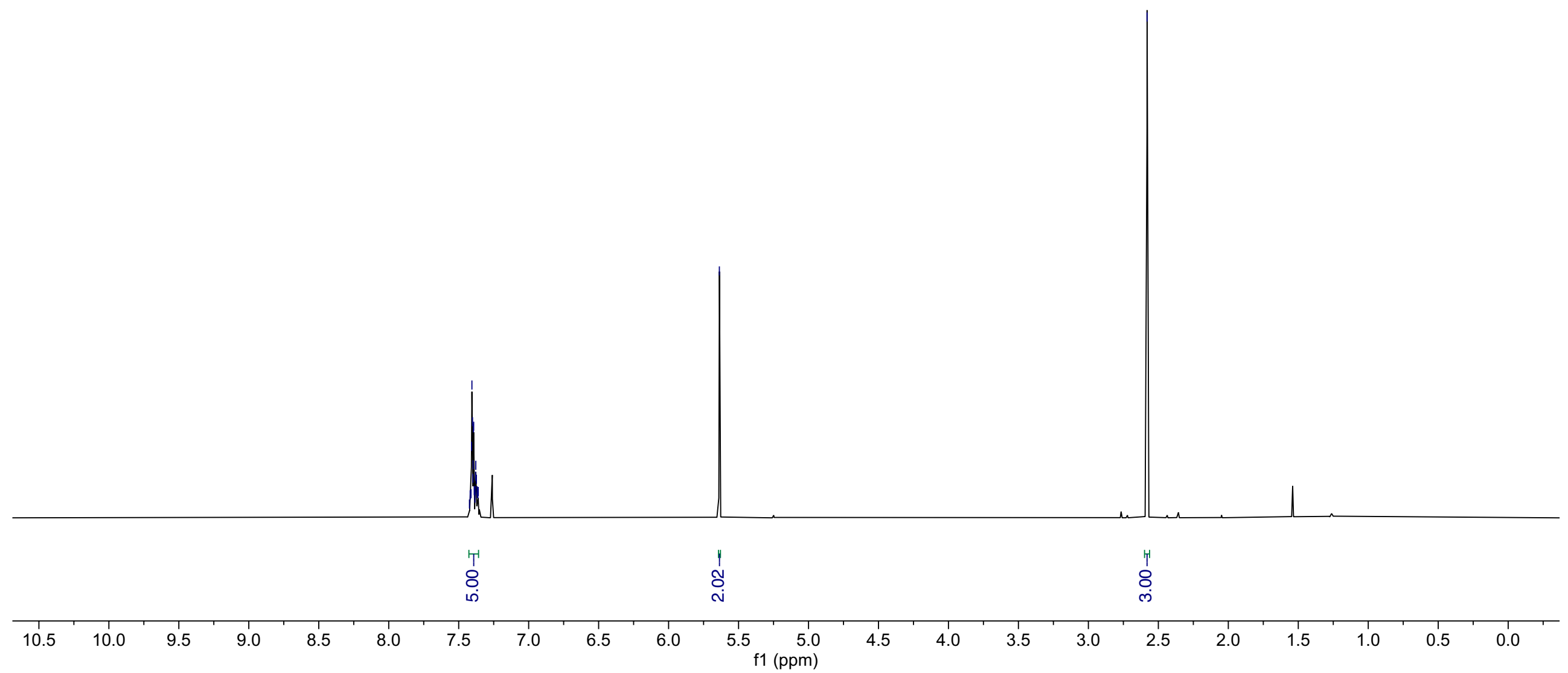


$126 \mathrm{MHz}, \mathrm{CDCl}_{3}$

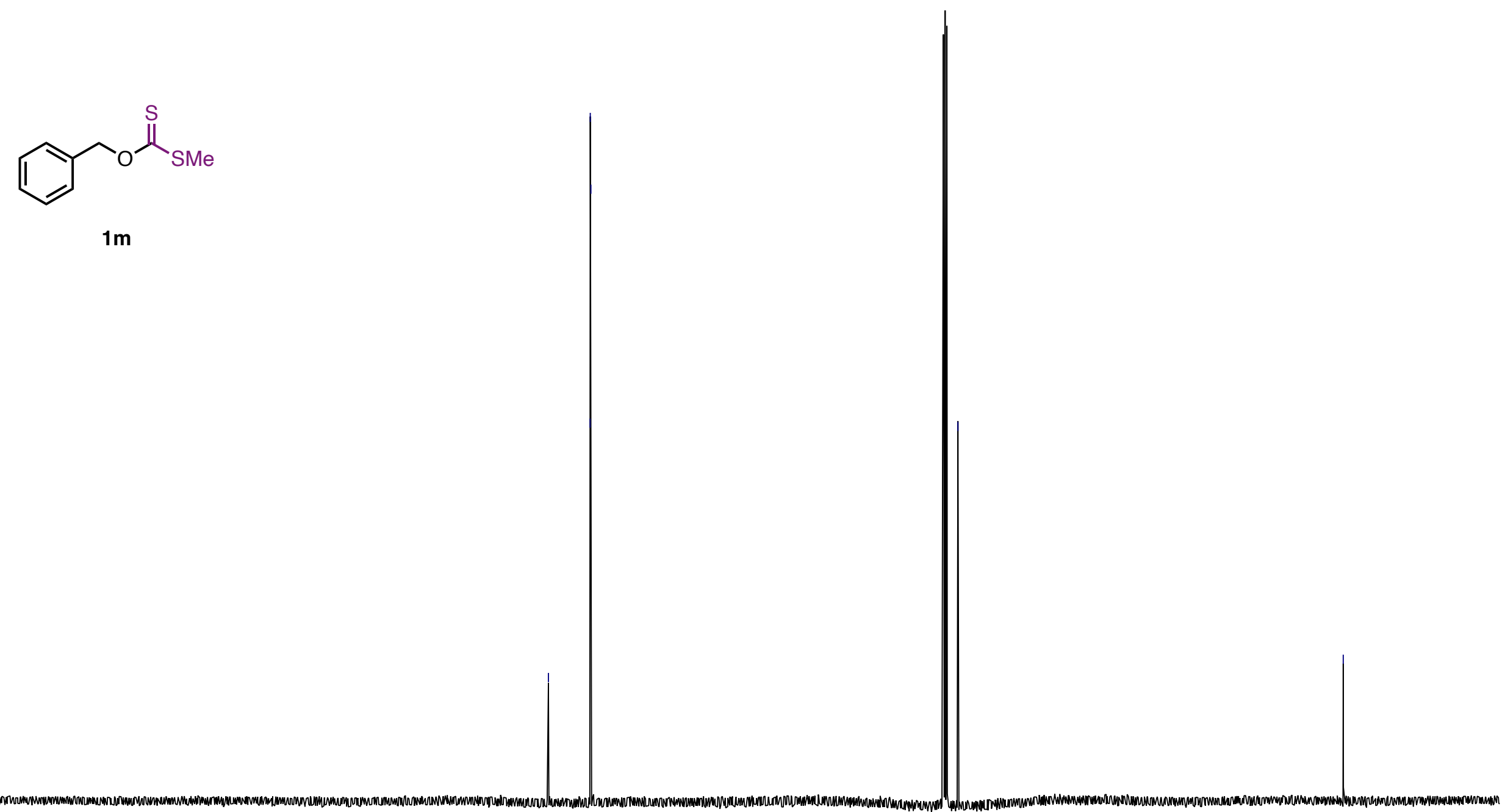


$500 \mathrm{MHz}, \mathrm{CDCl}_{3}$
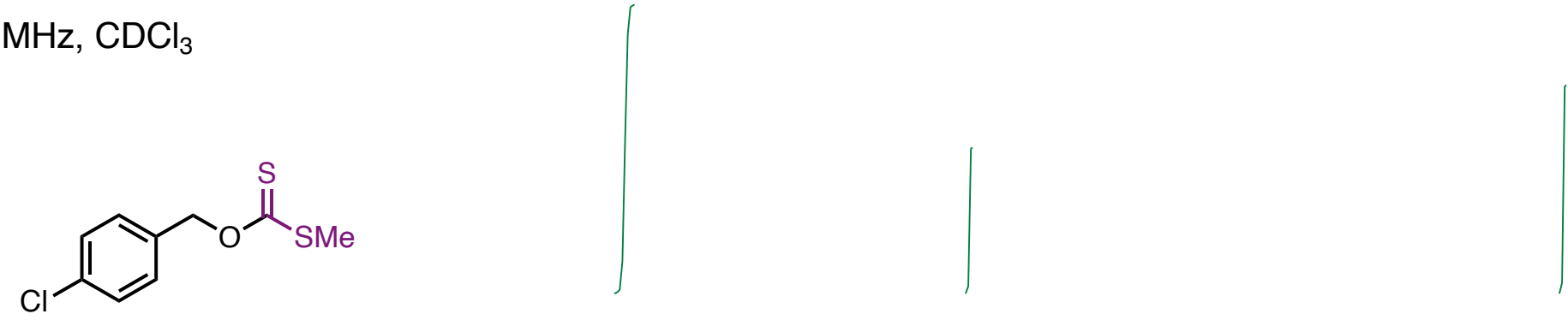

1n

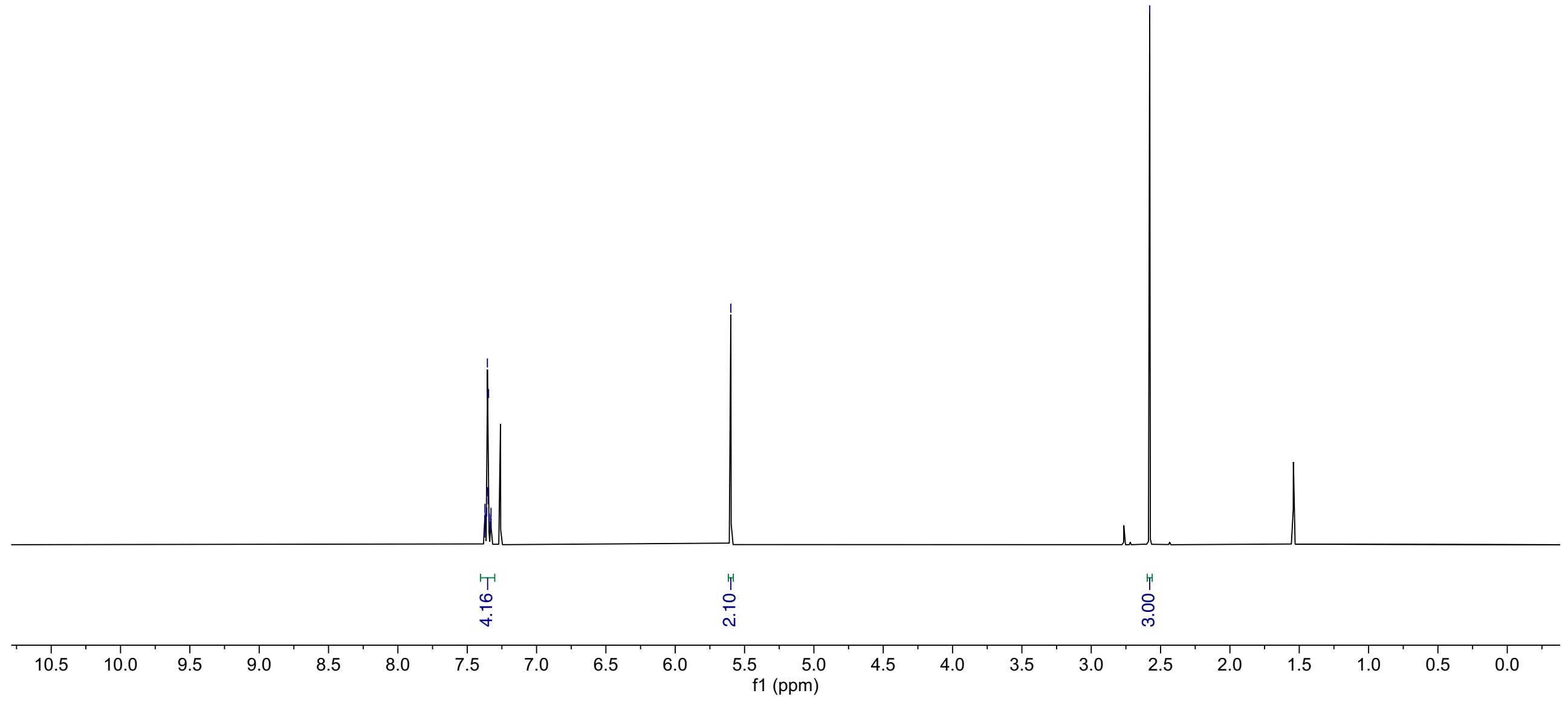


$126 \mathrm{MHz}, \mathrm{CDCl}_{3}$

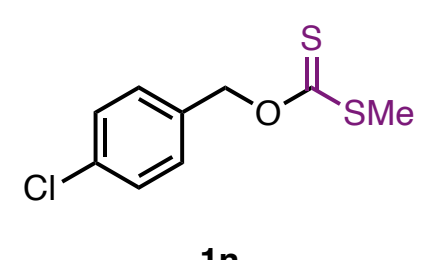

$1 n$

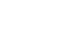


$500 \mathrm{MHz}, \mathrm{CDCl}_{3}$<smiles>CCOC(=O)C([14CH3])OC(=S)O[Na]</smiles>

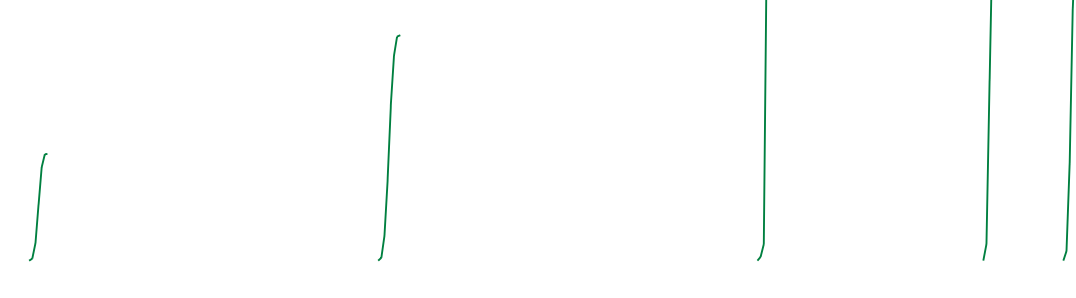

10

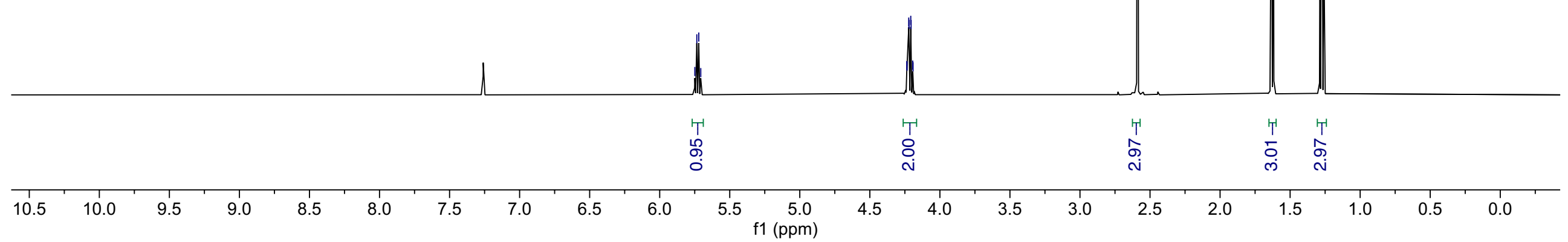


$126 \mathrm{MHz}, \mathrm{CDCl}_{3}$

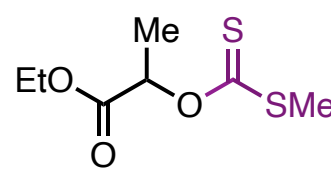

10

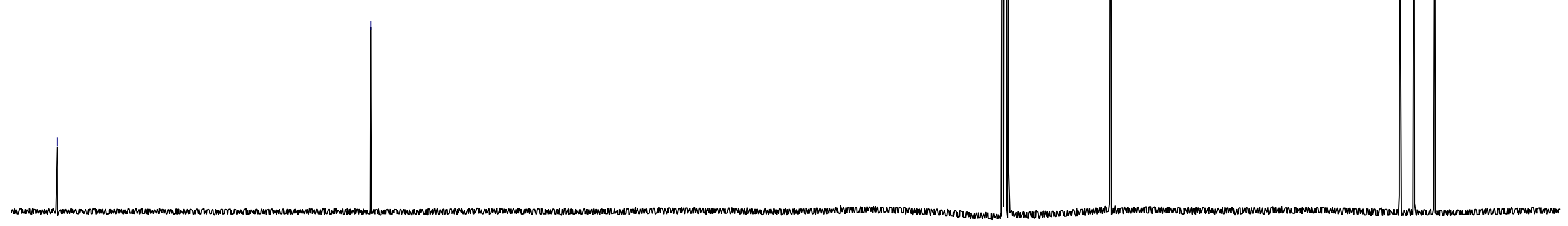

220

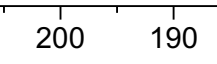

180

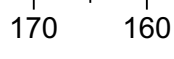

150

$140 \quad 130$

$\begin{array}{cc}120 & 110 \\ & \mathrm{f} 1(\mathrm{ppm})\end{array}$

90

$80 \quad 70 \quad 60$

$50 \quad 40$

30

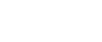


$400 \mathrm{MHz}, \mathrm{CDCl}_{3}$<smiles>CCN(CC(C)C)C(=S)n1ccnc1</smiles>

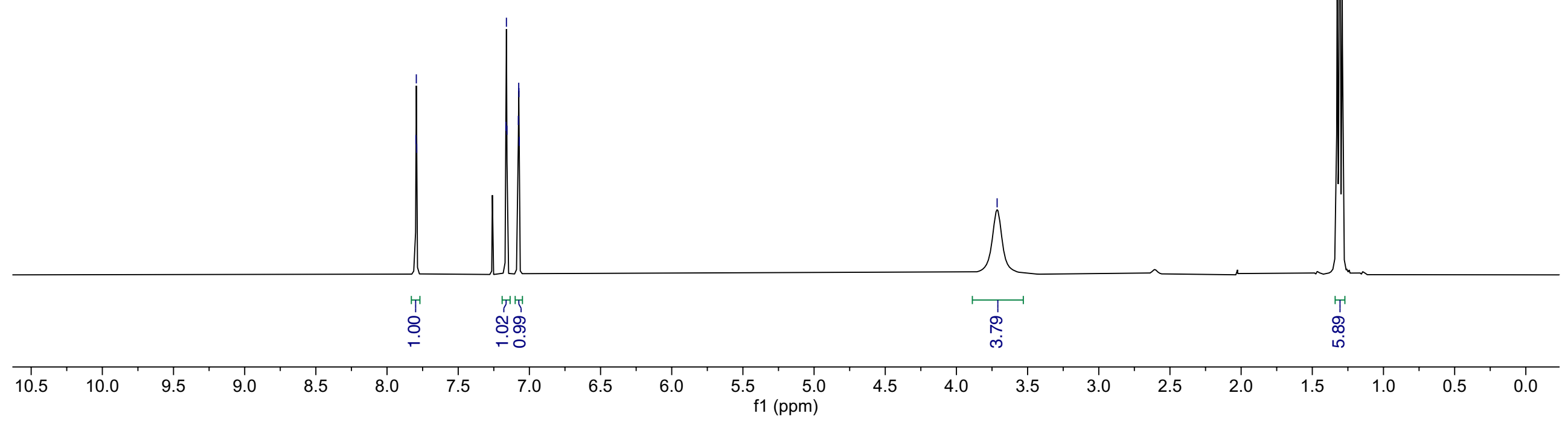




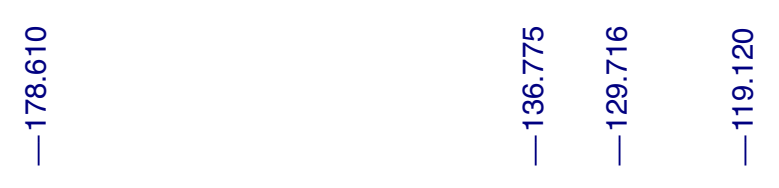

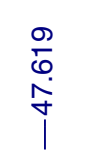

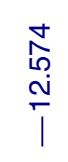

$101 \mathrm{MHz}, \mathrm{CDCl}_{3}$<smiles>CCN(CC)C(=S)n1ccnc1</smiles>

$4 a$

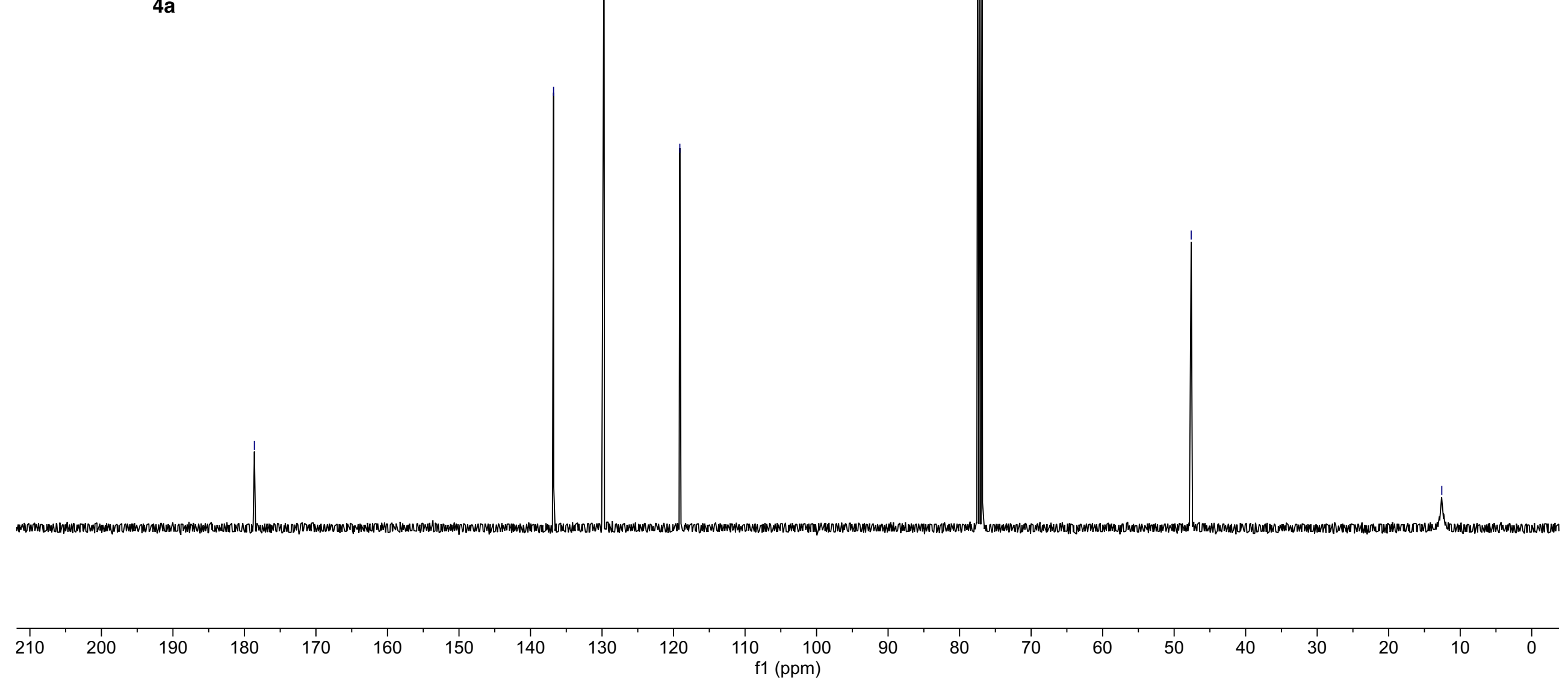

S148 
$500 \mathrm{MHz}, \mathrm{CDCl}_{3}$<smiles>C=CCN(CC=C)C(=S)n1ccnc1</smiles>

4b
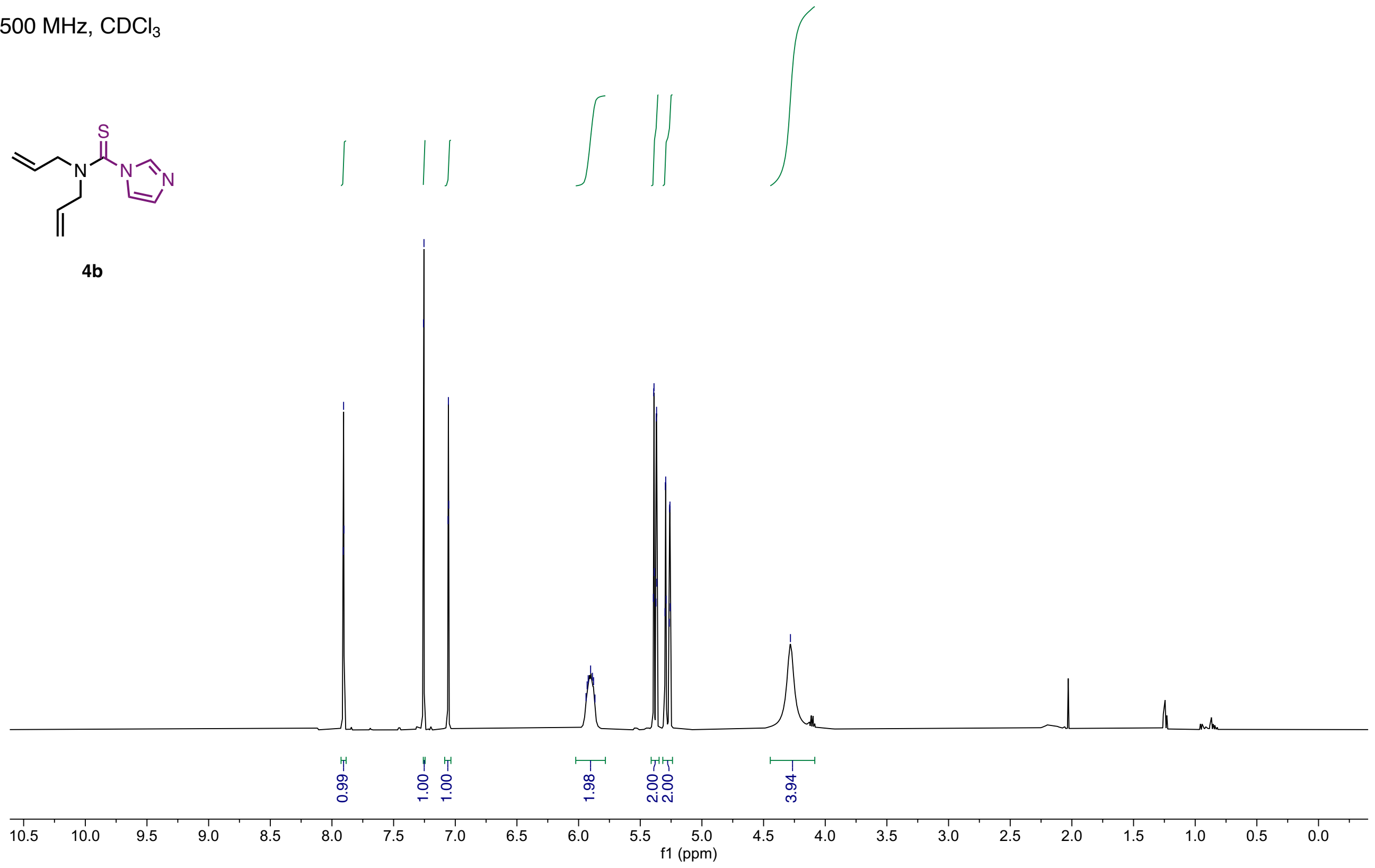
$126 \mathrm{MHz}, \mathrm{CDCl}_{3}$

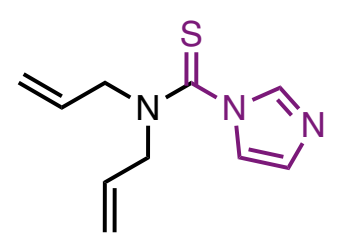

4b
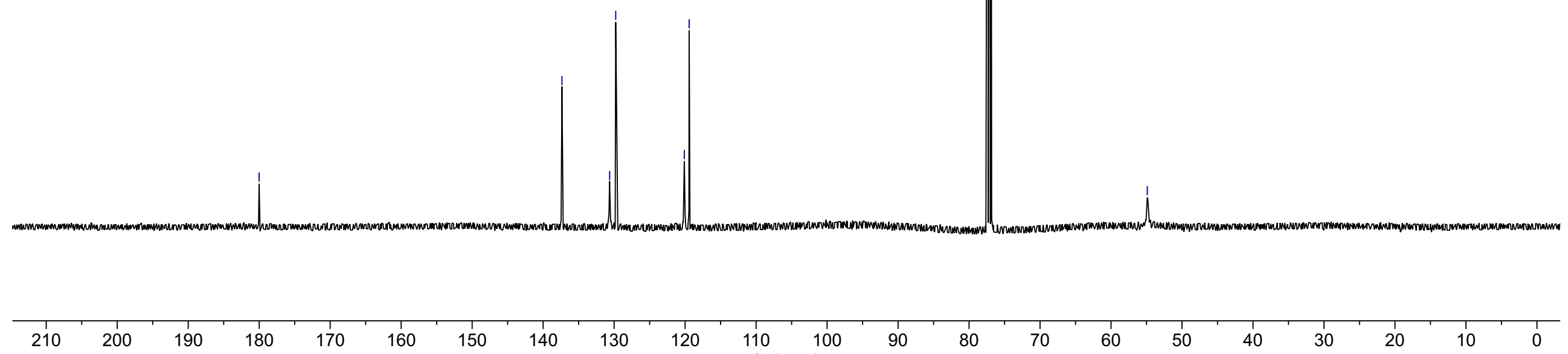

$130 \quad 120$

$110 \quad 100$

90

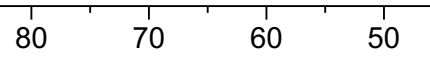

$40 \quad 30$

20

$1 0 \longdiv { 1 }$ 
$400 \mathrm{MHz}, \mathrm{CDCl}_{3}$

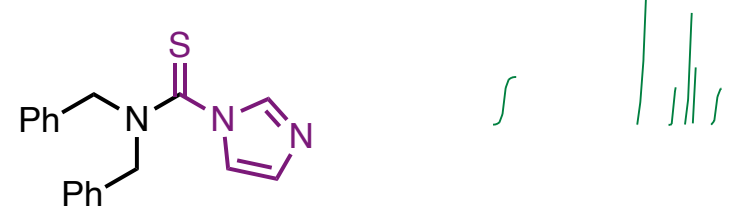

4c

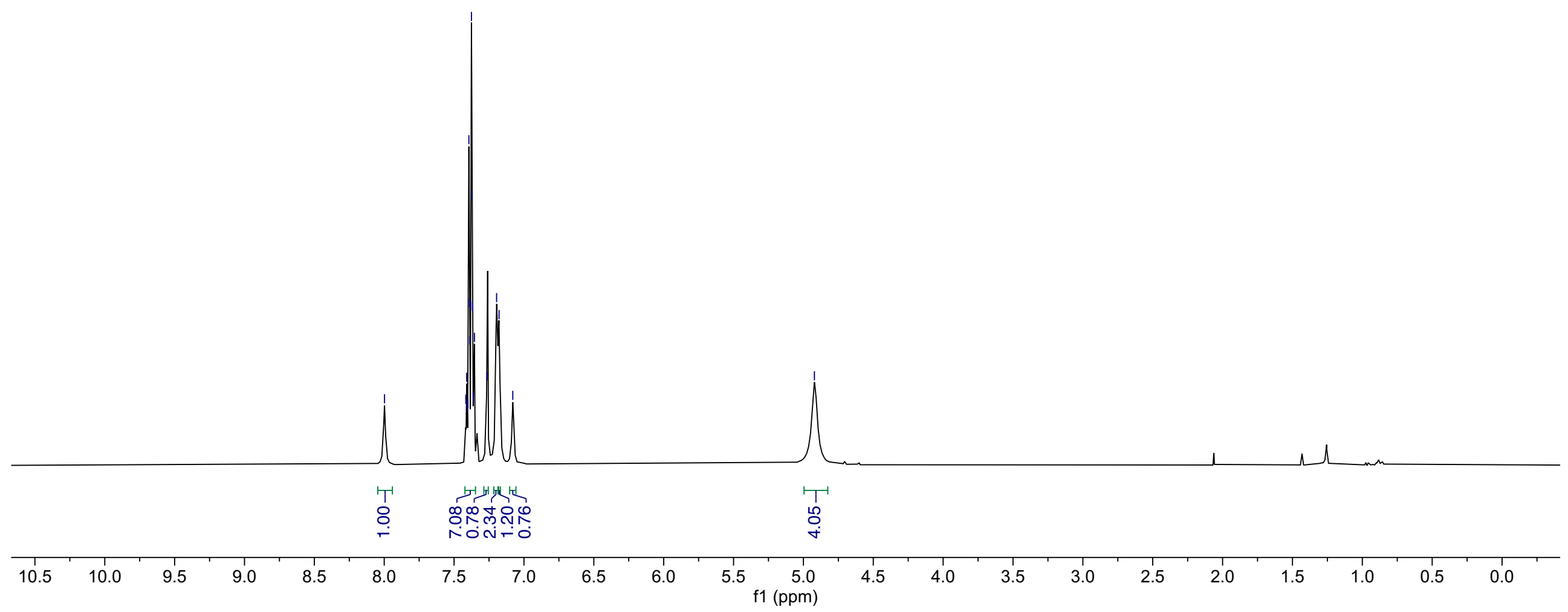


$100 \mathrm{MHz}, \mathrm{CDCl}_{3}$

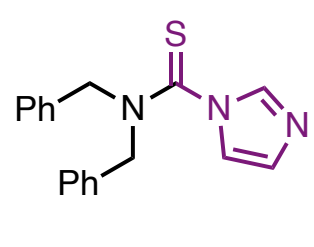

4c

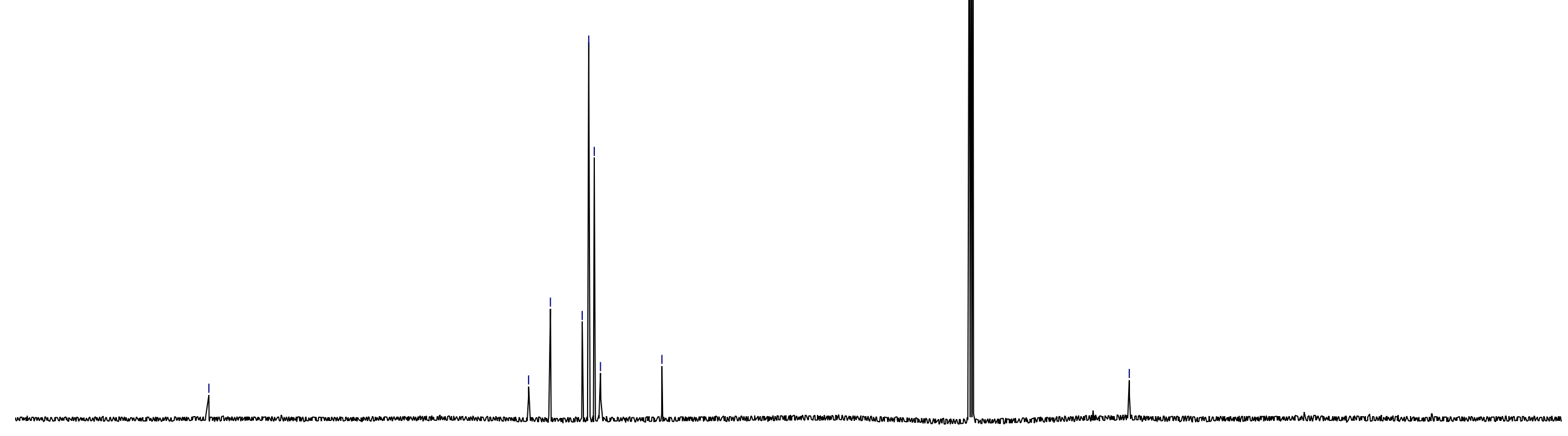


$500 \mathrm{MHz}, \mathrm{CDCl}_{3}$<smiles>S=C(N1CCCC1)n1ccnc1</smiles>

4d

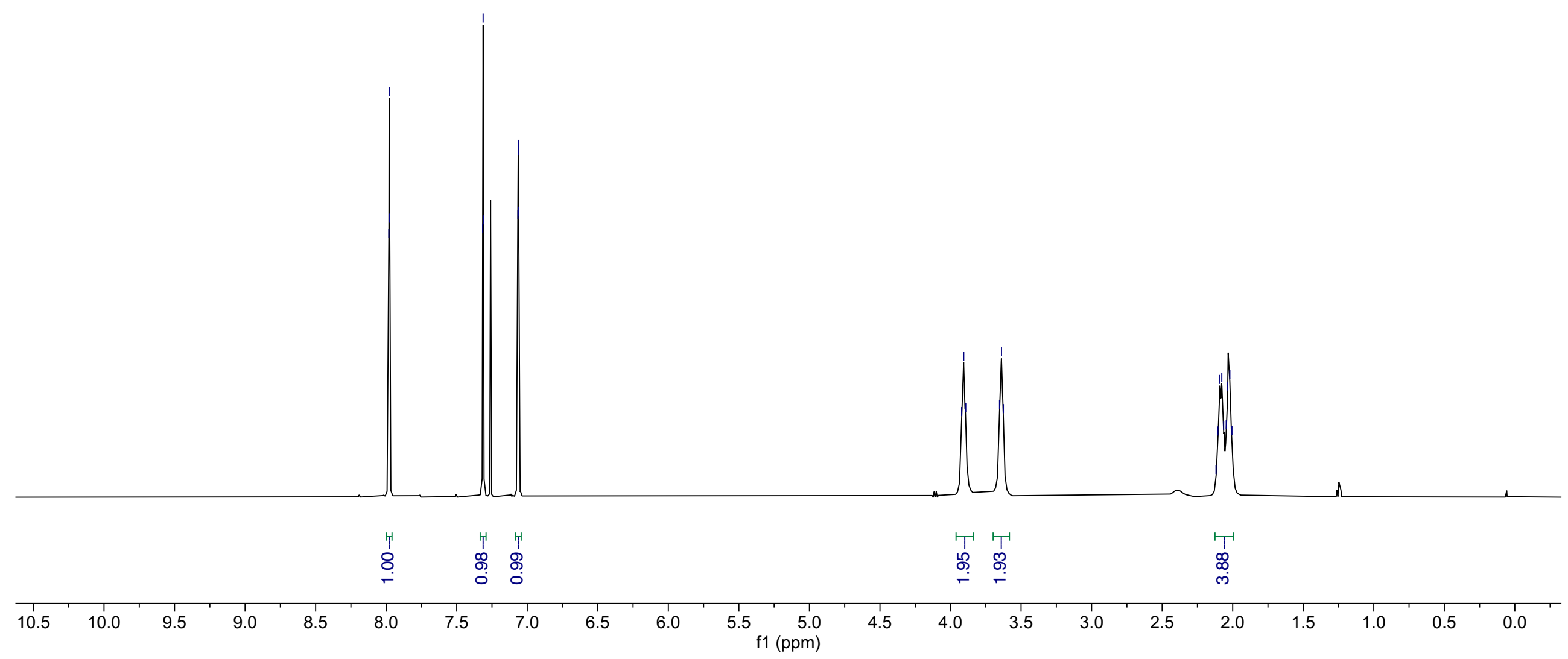


$126 \mathrm{MHz}, \mathrm{CDCl}_{3}$

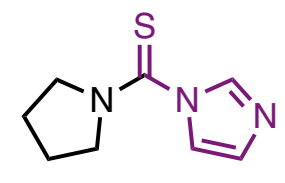

4d

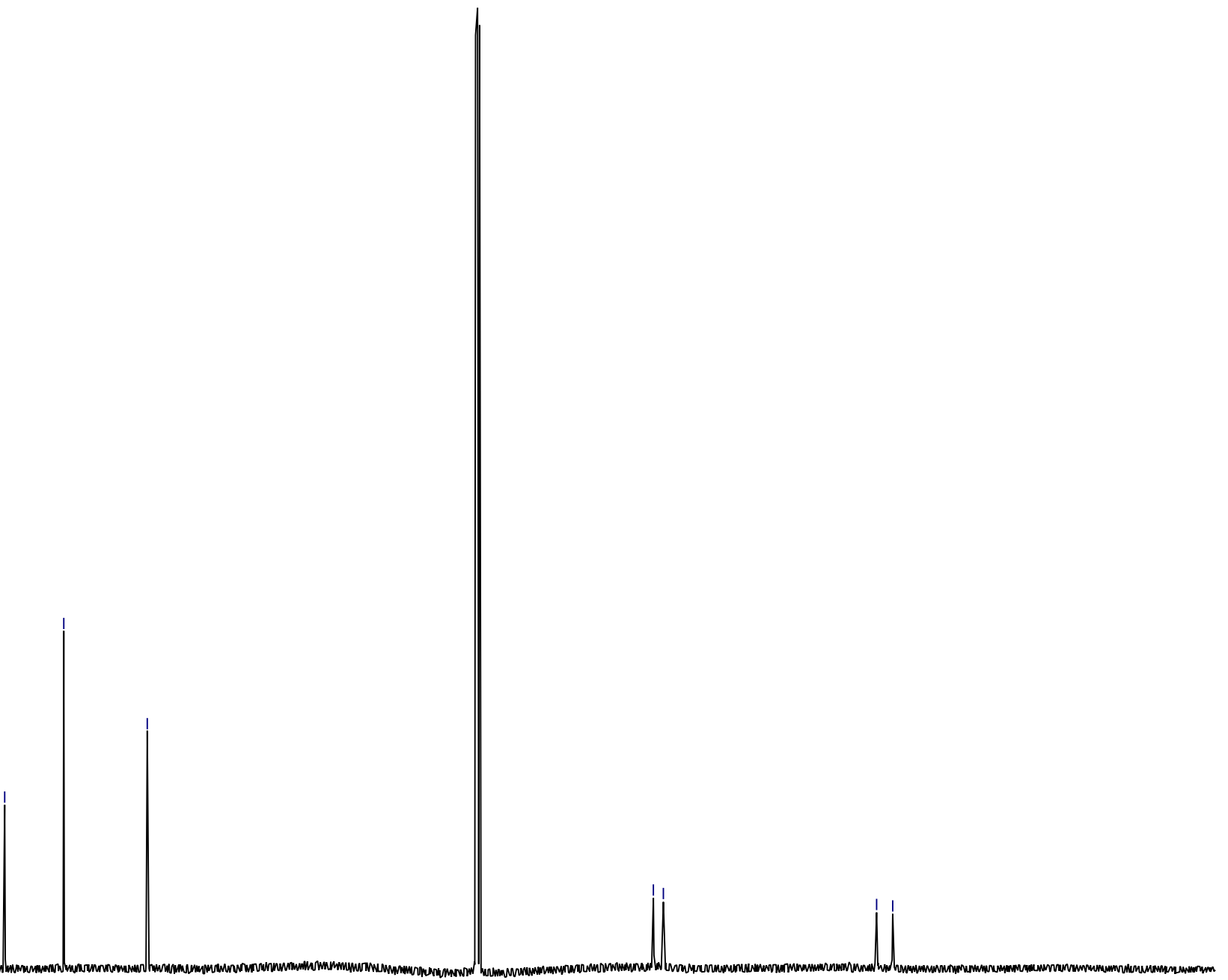




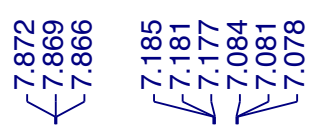

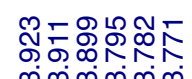

$400 \mathrm{MHz}, \mathrm{CDCl}_{3}$<smiles>S=C(N1CCOCC1)n1ccnc1</smiles>

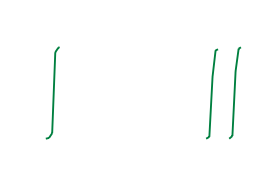

$4 e$

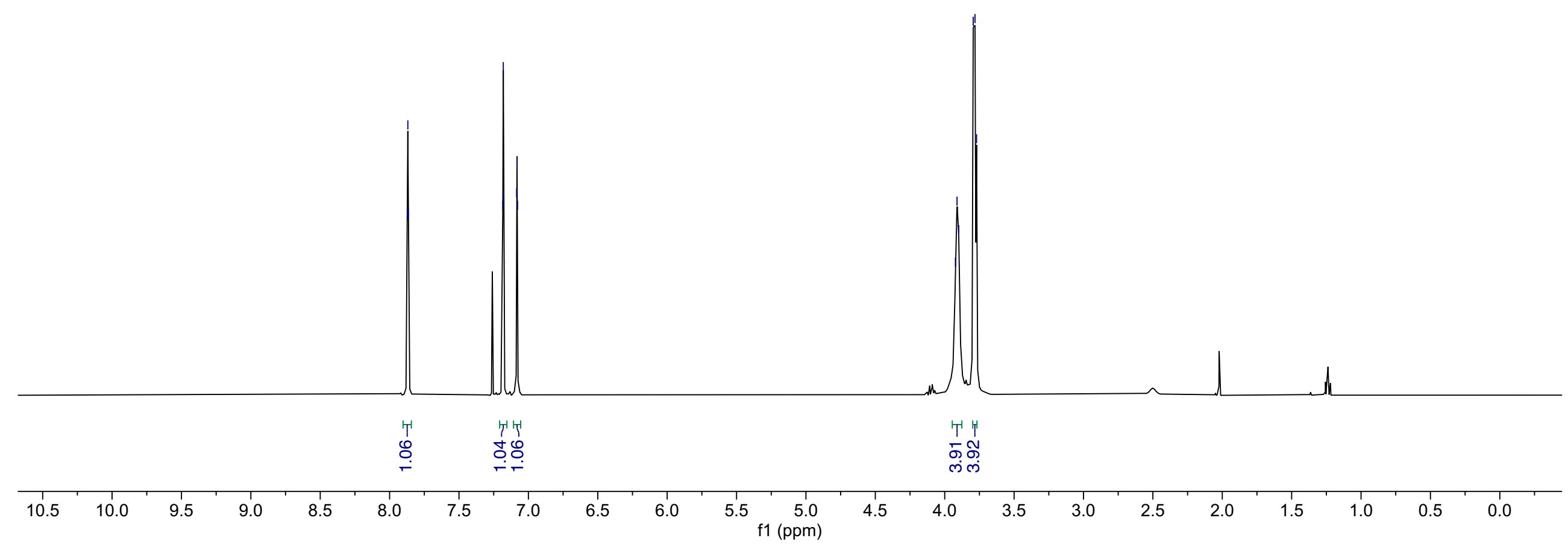

S155 


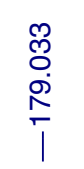

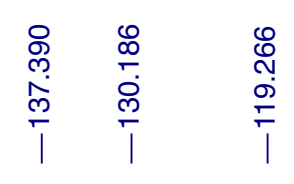

$\begin{array}{ll}8 & 0 \\ 0 & 0 \\ 0 & 0 \\ 0 & 0 \\ 0 & 1\end{array}$

$100 \mathrm{MHz}, \mathrm{CDCl}_{3}$<smiles>S=C(N1CCOCC1)n1ccnc1</smiles>

$4 e$
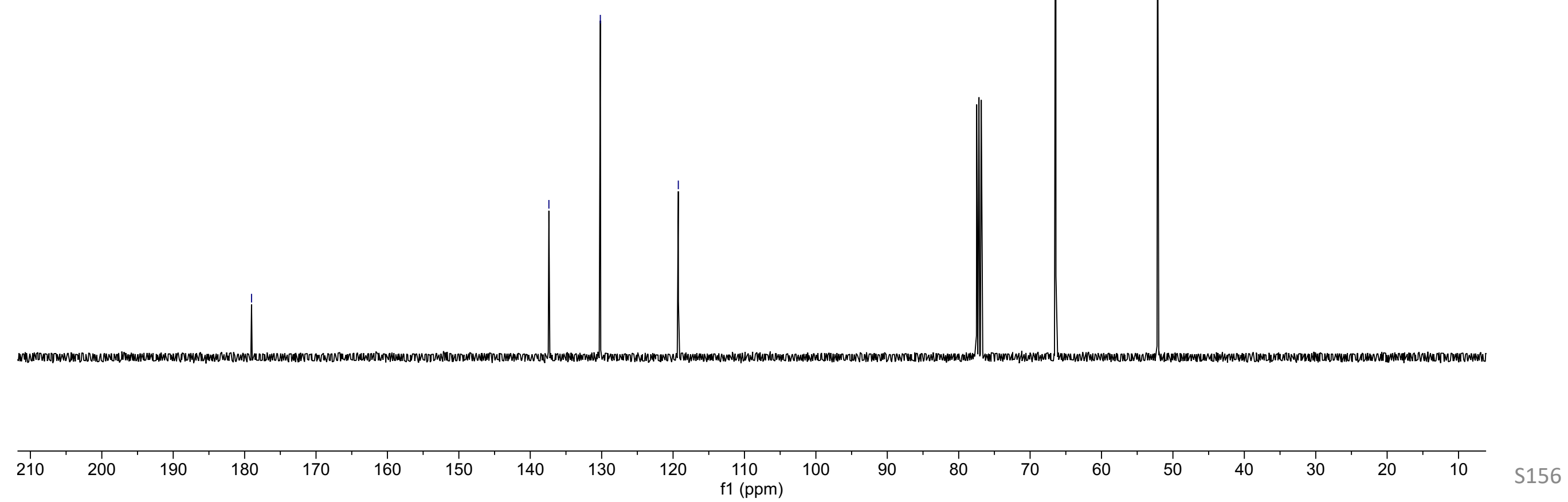
$100 \mathrm{MHz}, \mathrm{CDCl}_{3}$

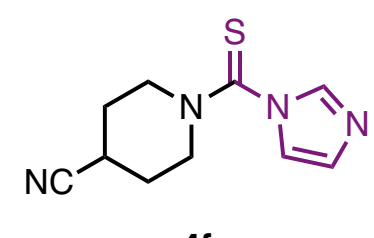

4f

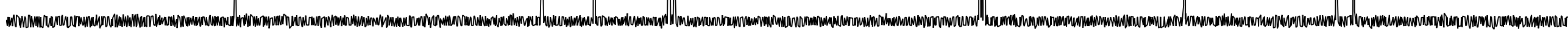

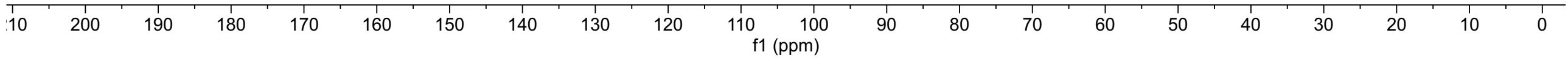

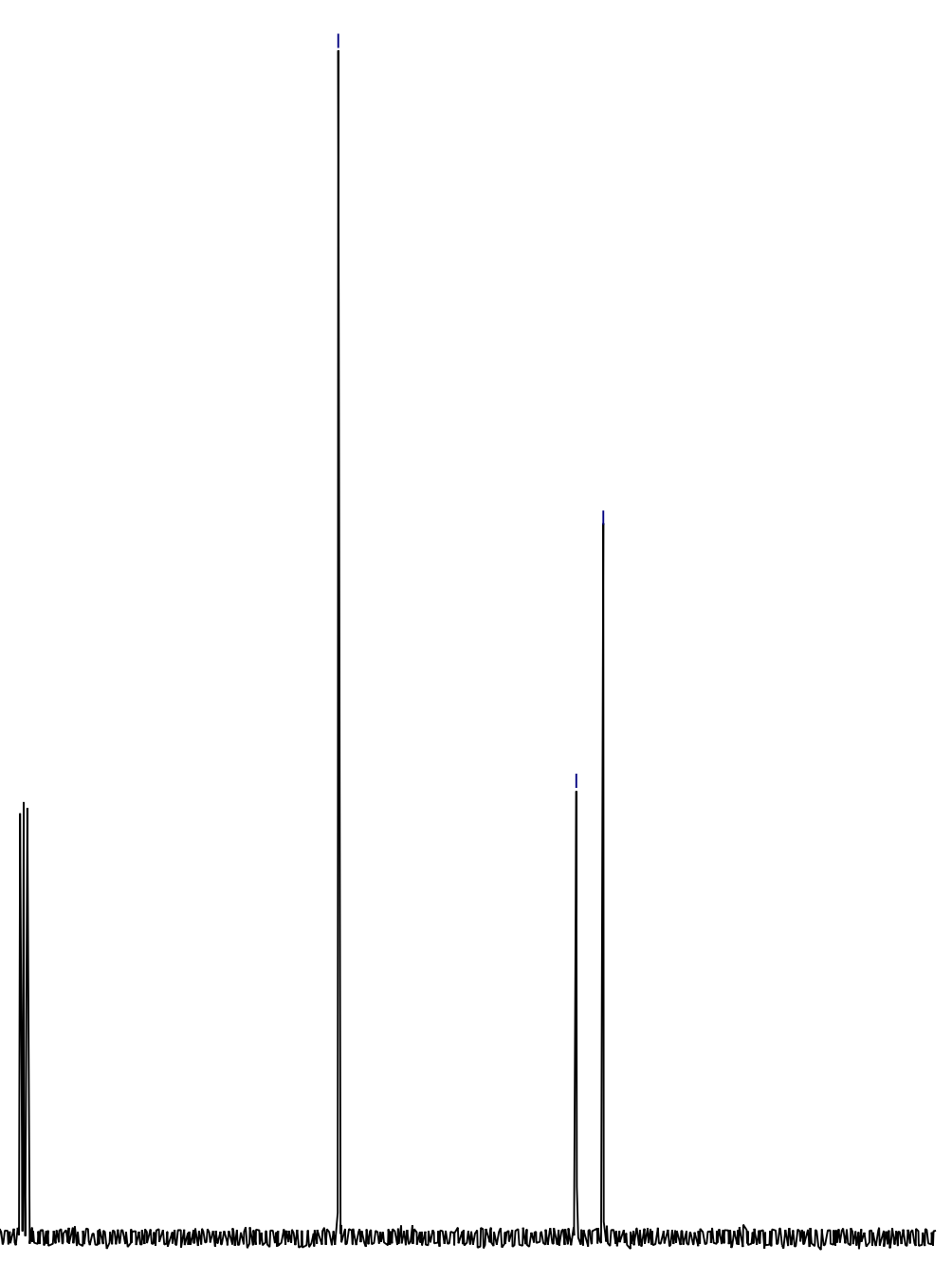


$400 \mathrm{MHz}, \mathrm{CDCl}_{3}$

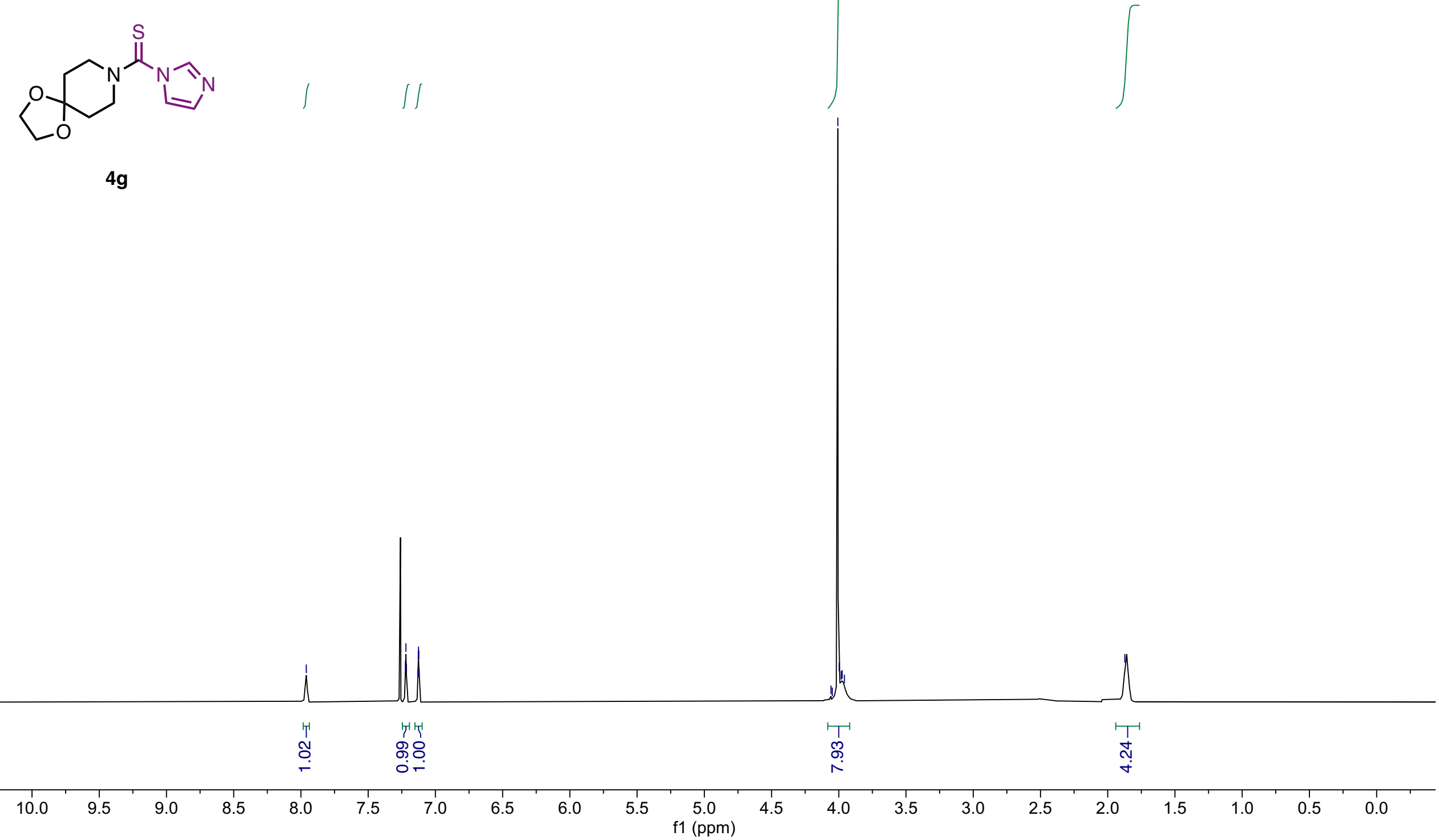




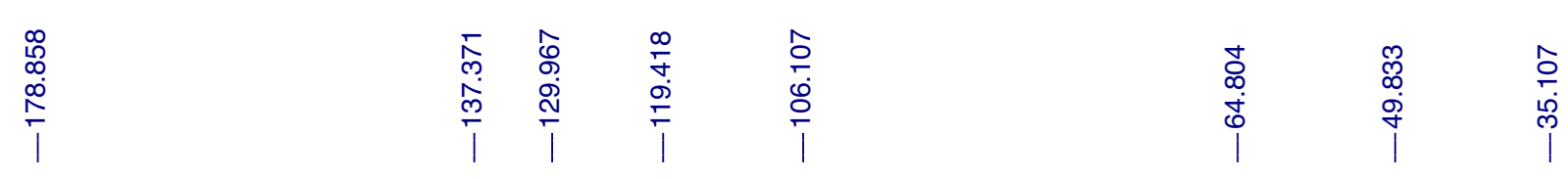

$101 \mathrm{MHz}, \mathrm{CDCl}_{3}$

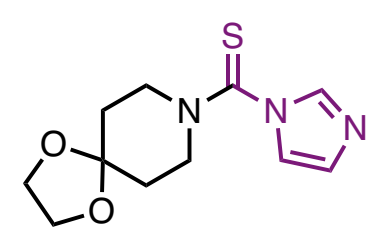

$4 g$

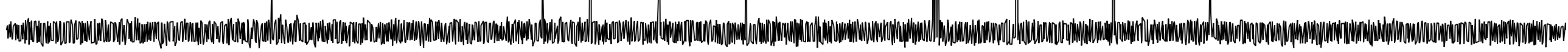

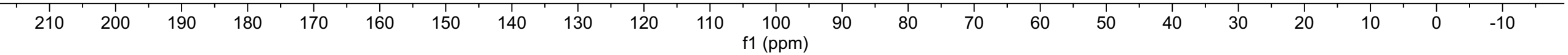




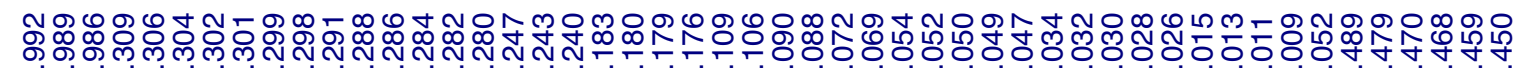

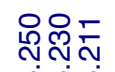

$400 \mathrm{MHz}, \mathrm{CDCl}_{3}$
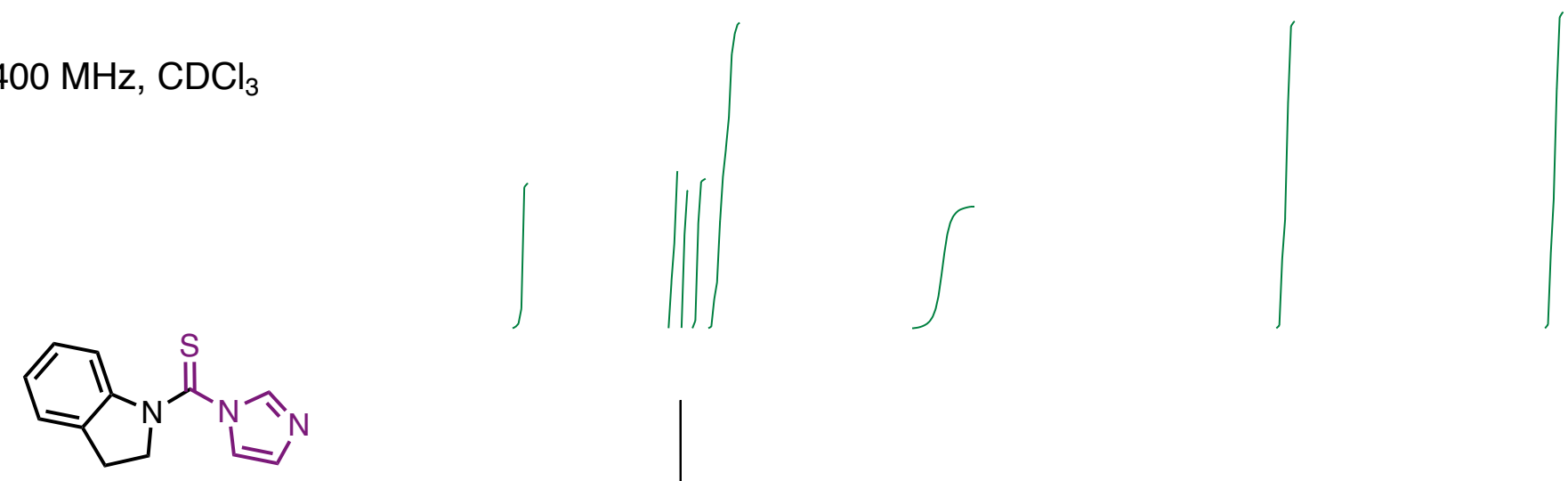

4h

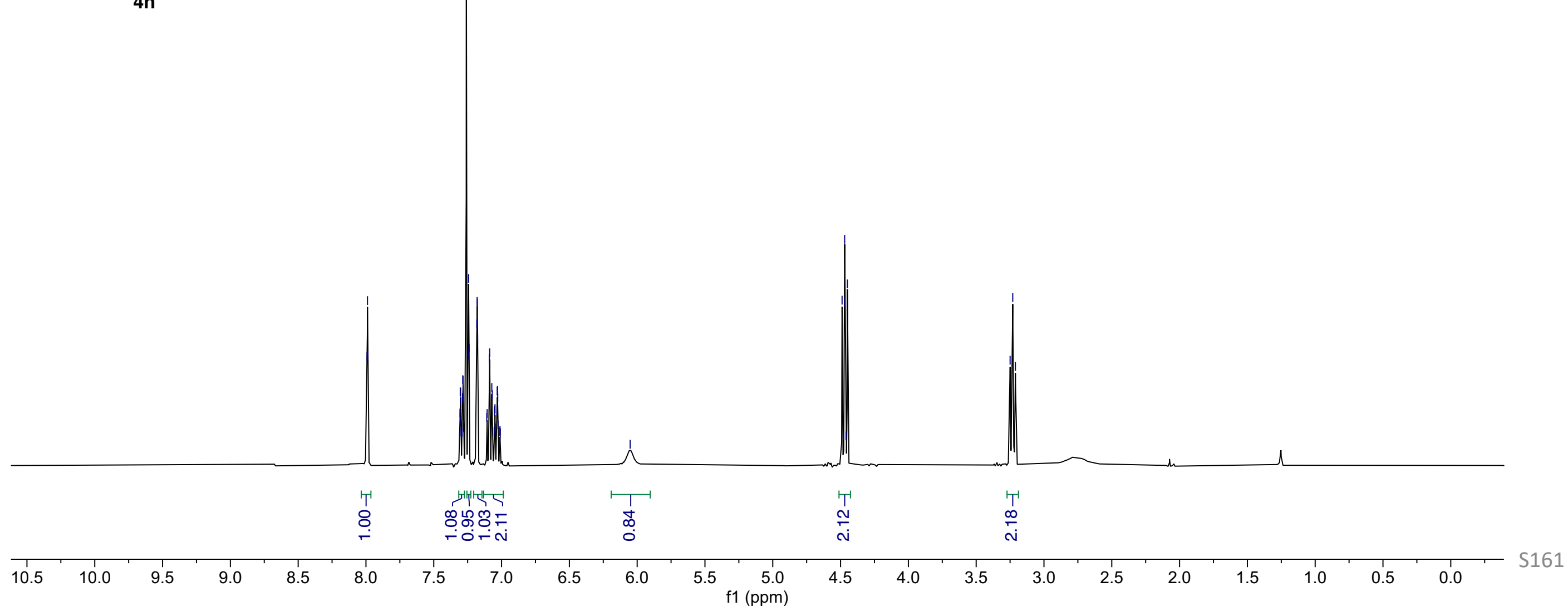


$\underset{\substack{\text { ? } \\ \text { ำ }}}{\stackrel{1}{1}}$

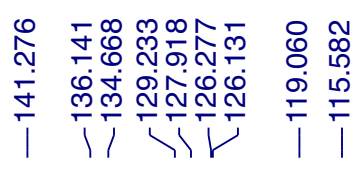

مَ

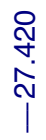

$101 \mathrm{MHz}, \mathrm{CDCl}_{3}$<smiles>S=C(N1CCc2ccccc21)n1ccnc1</smiles>
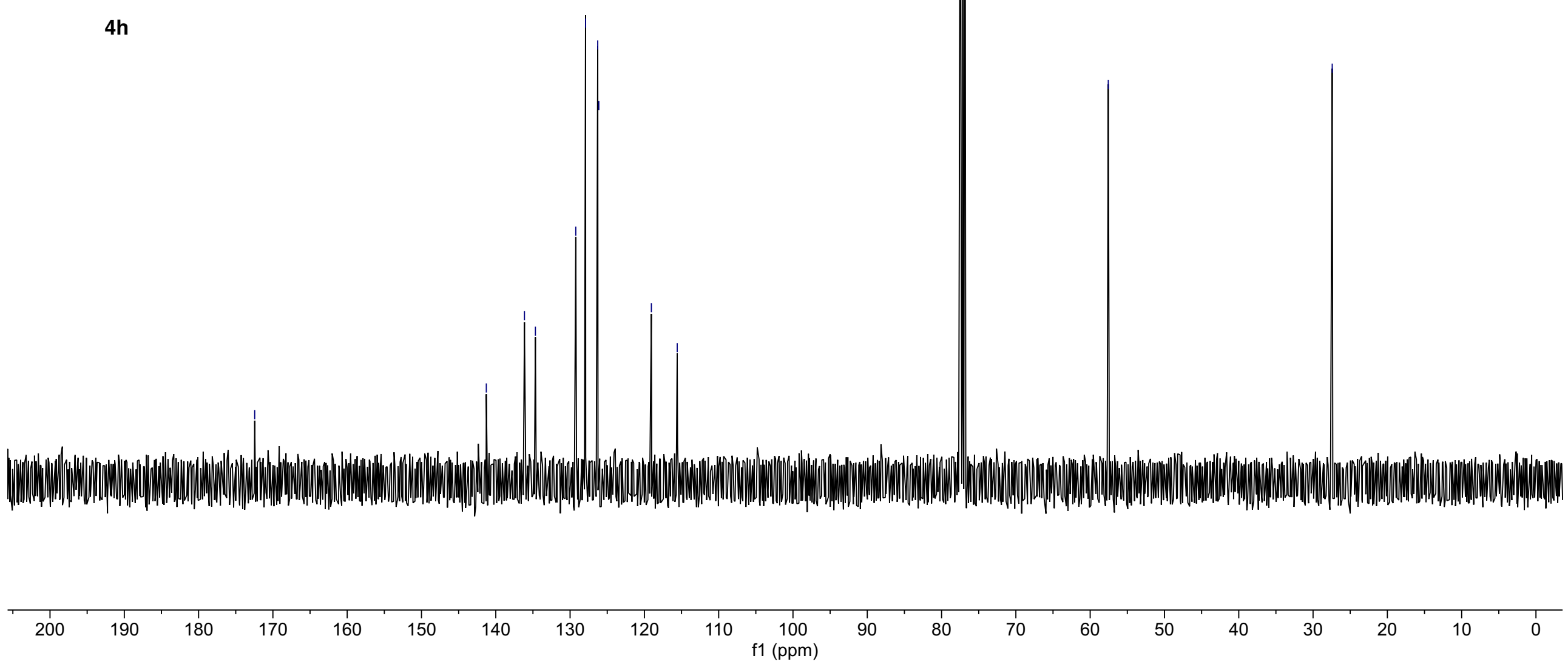

S162 
$400 \mathrm{MHz}, \mathrm{CDCl}_{3}$
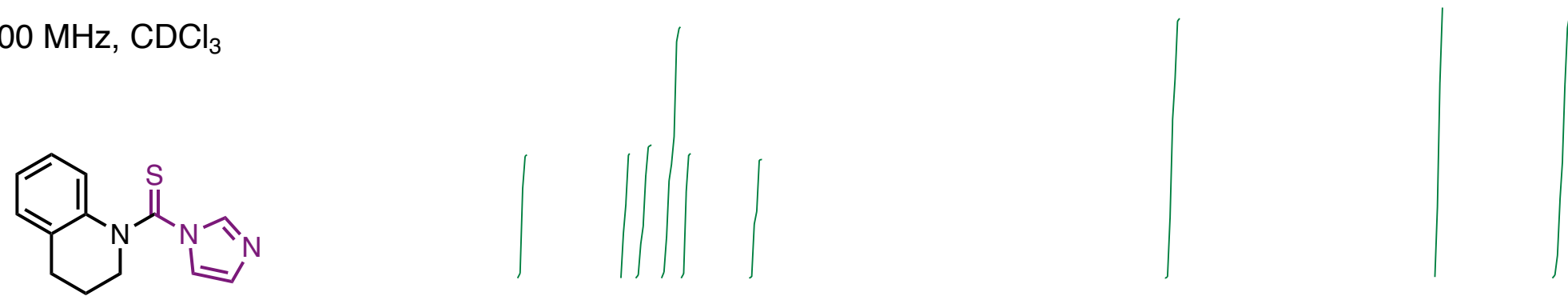

$4 \mathbf{i}$

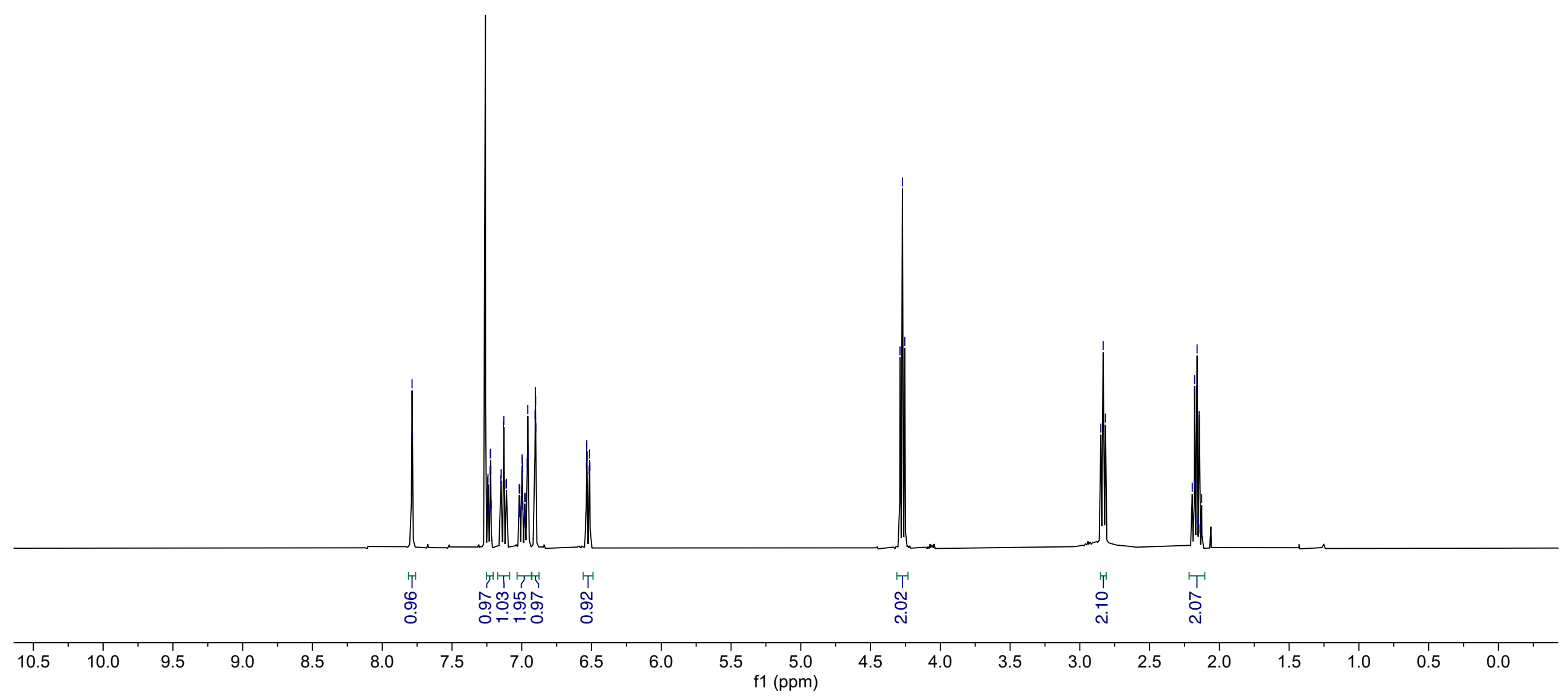




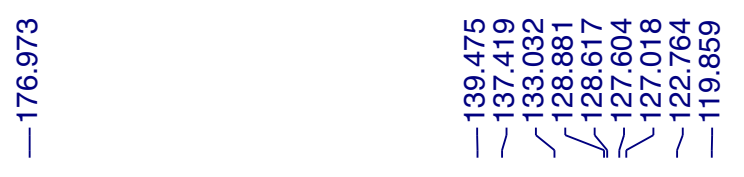

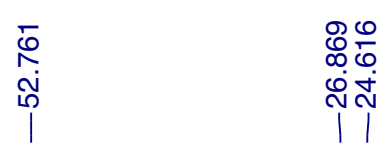

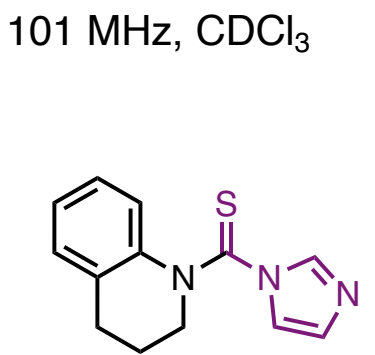

$4 i$

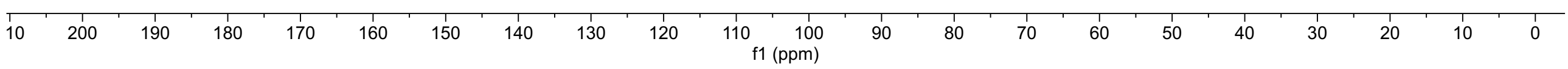

S164 
$400 \mathrm{MHz}, \mathrm{CDCl}_{3}$<smiles>S=C(N1CCCCCC1)n1ccnc1</smiles>
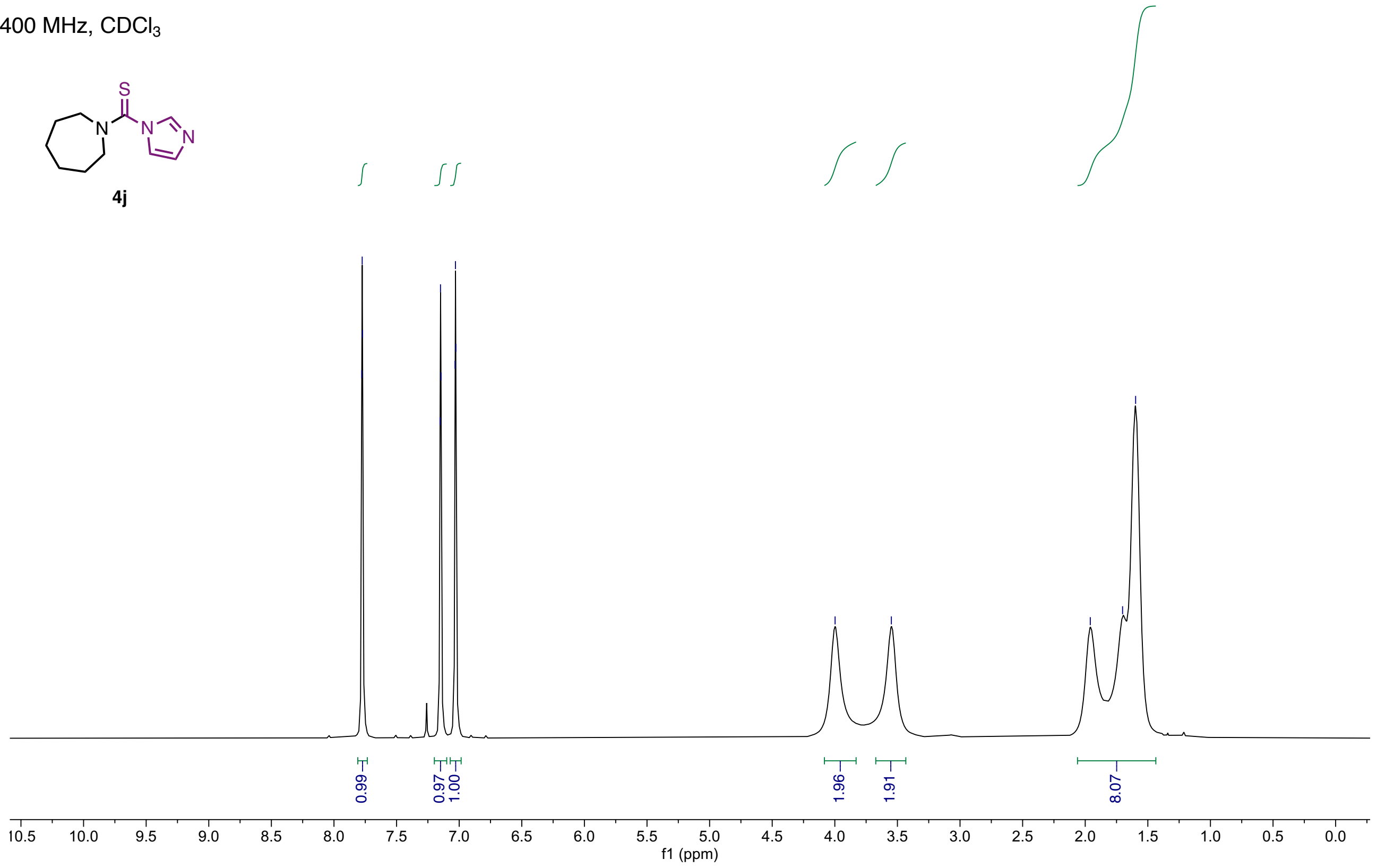


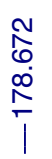

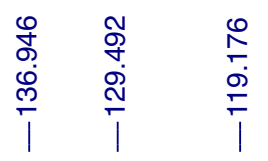

20
80
010
10
10

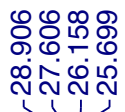

$101 \mathrm{MHz}, \mathrm{CDCl}_{3}$<smiles>S=C(N1CCCCCC1)n1ccnc1</smiles>

4j
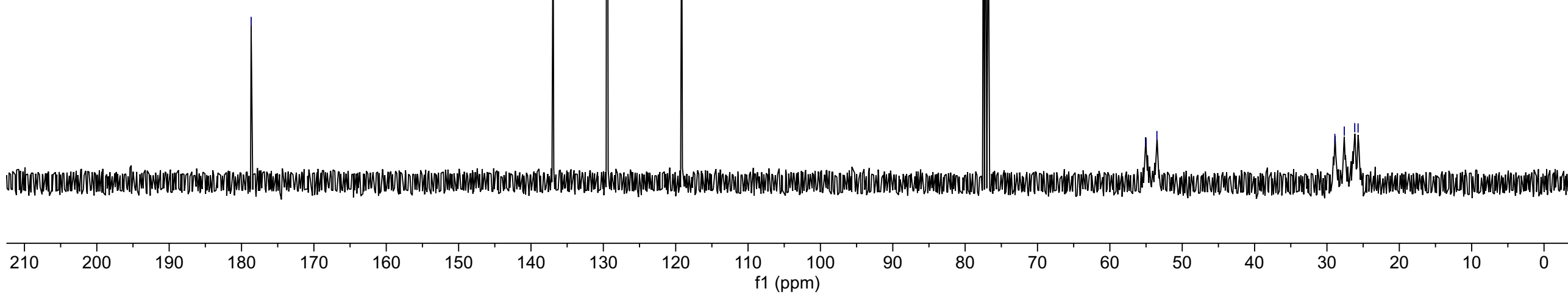

S166 
$400 \mathrm{MHz}, \mathrm{CDCl}_{3}$<smiles>COCCSC(=S)n1ccnc1</smiles>

$4 k$

$$
\text { k }
$$
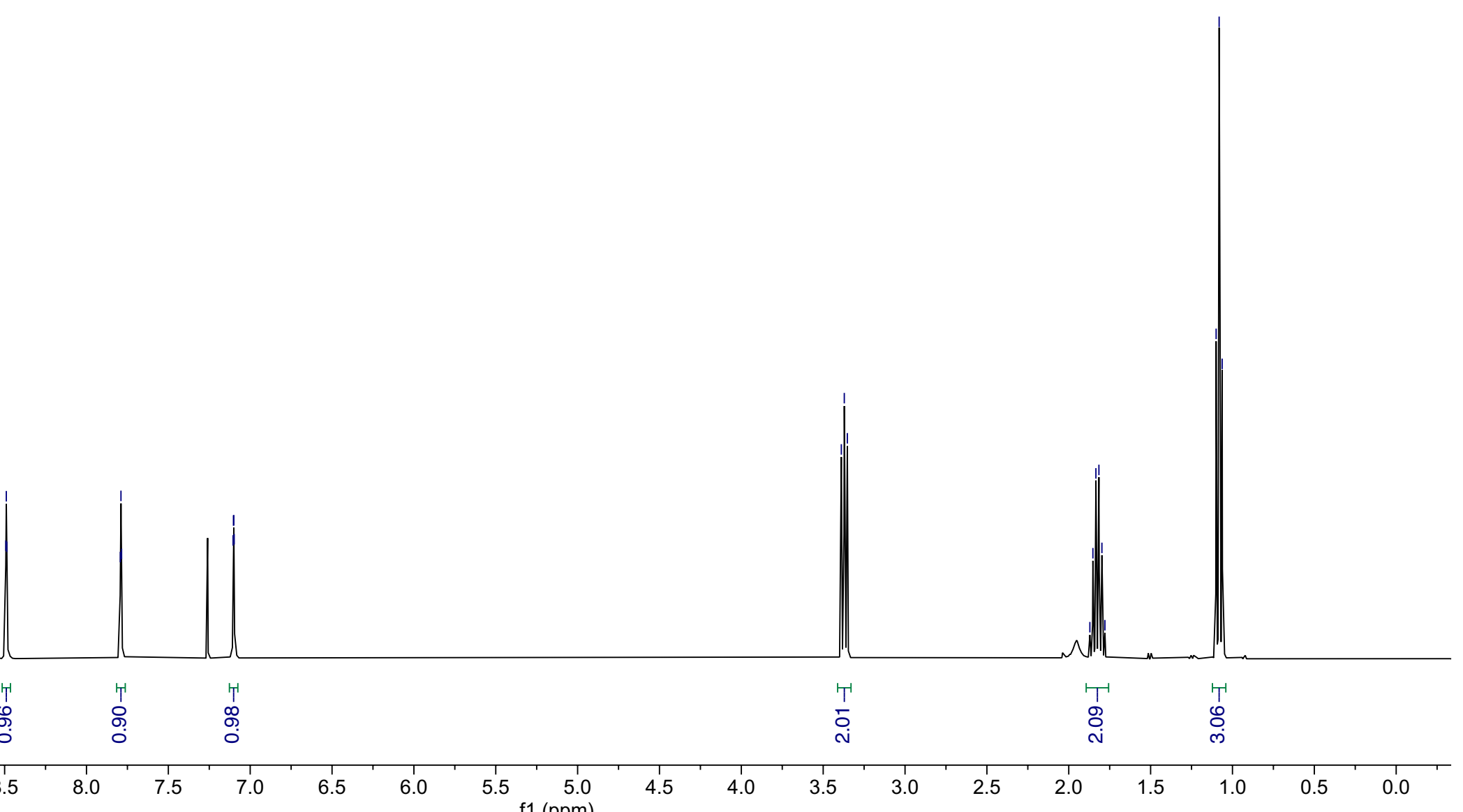


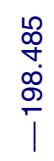

号

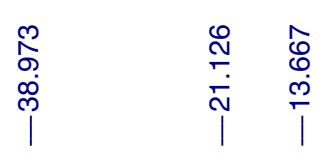

$101 \mathrm{MHz}, \mathrm{CDCl}_{3}$
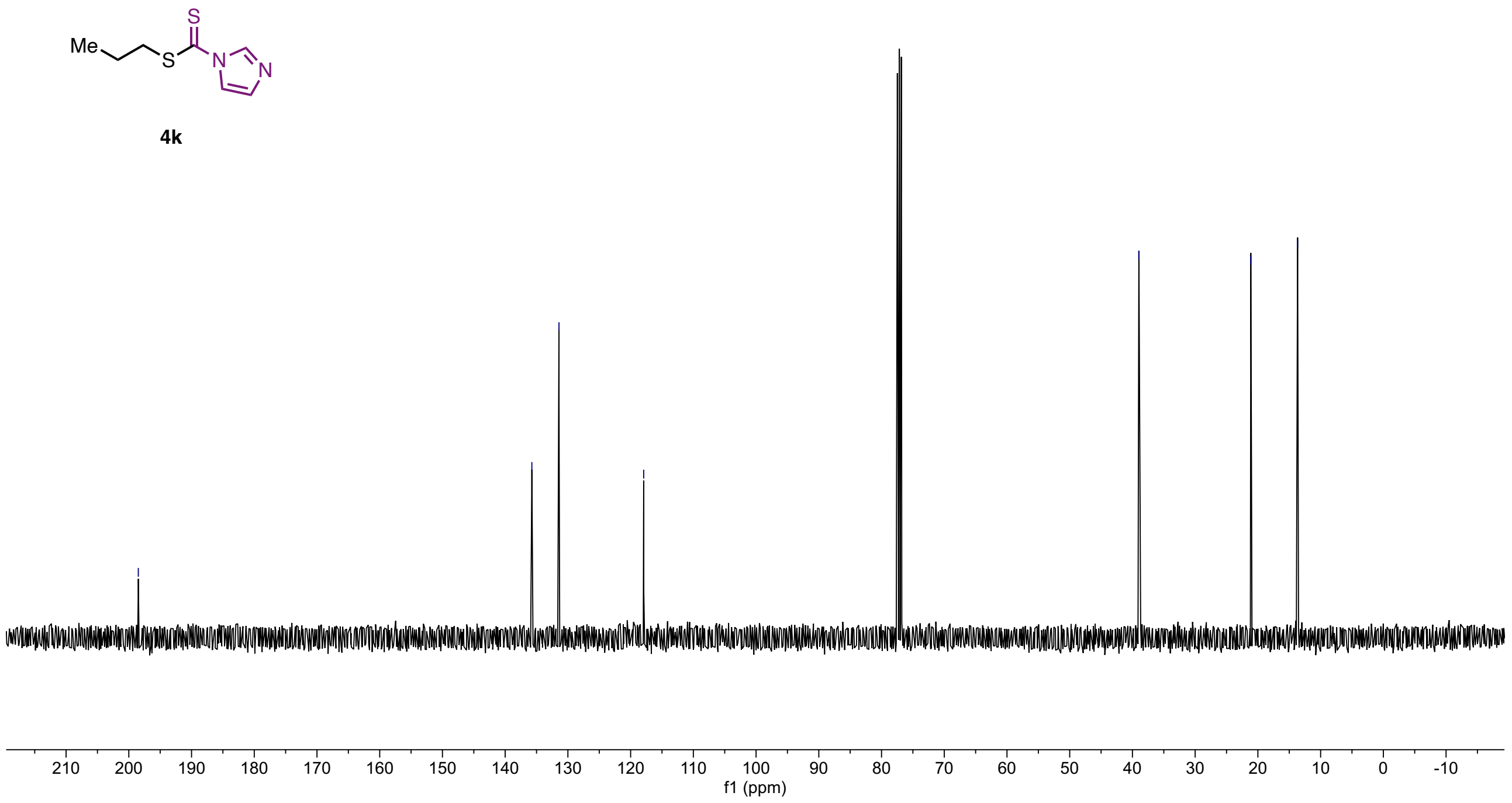

S168 
$400 \mathrm{MHz}, \mathrm{CDCl}_{3}$<smiles>S=C(OCCCc1ccccc1)n1ccnc1</smiles>

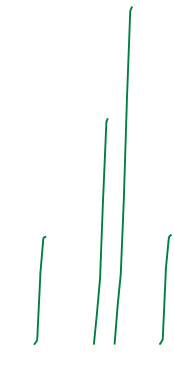

4

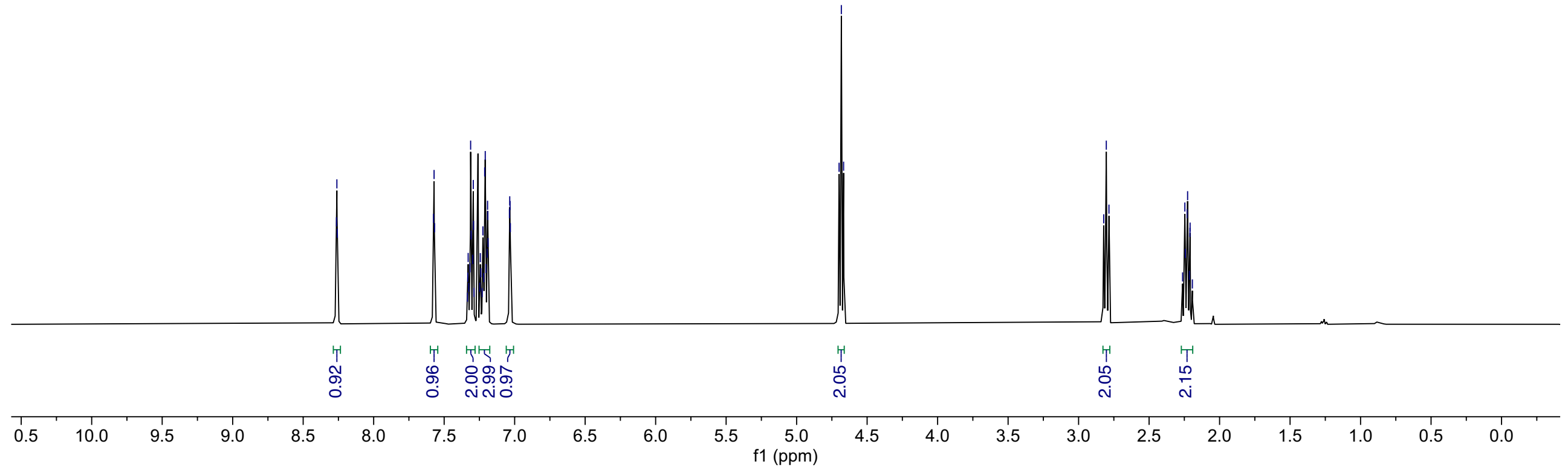


$101 \mathrm{MHz}, \mathrm{CDCl}_{3}$

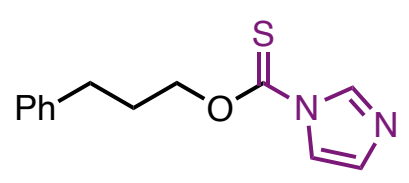

4

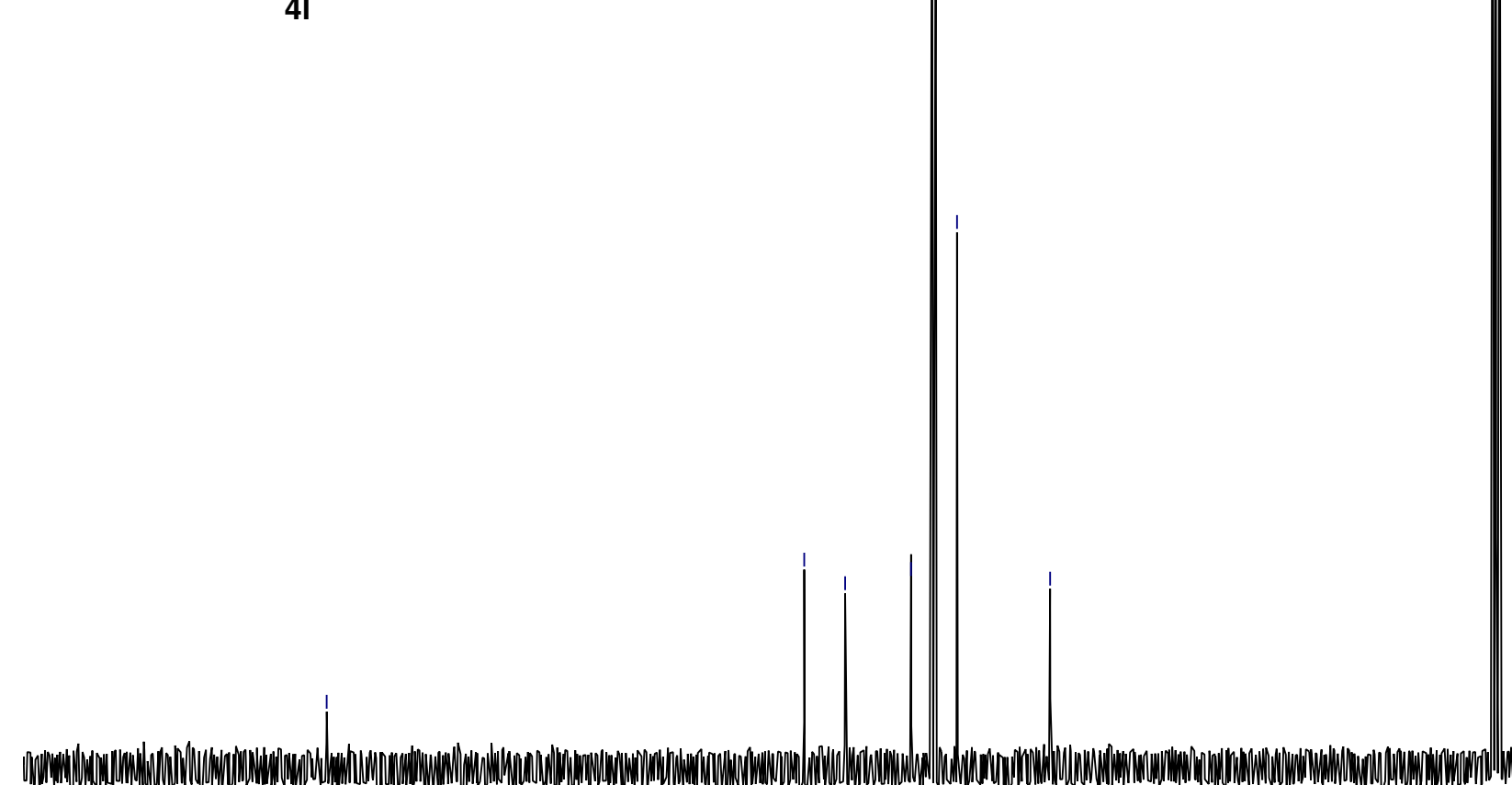

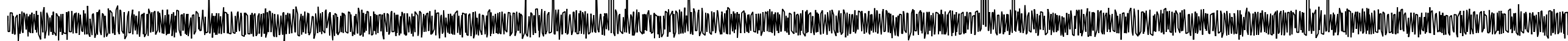

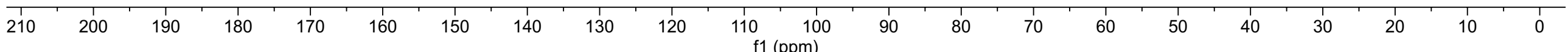


$400 \mathrm{MHz}, \mathrm{CDCl}_{3}$
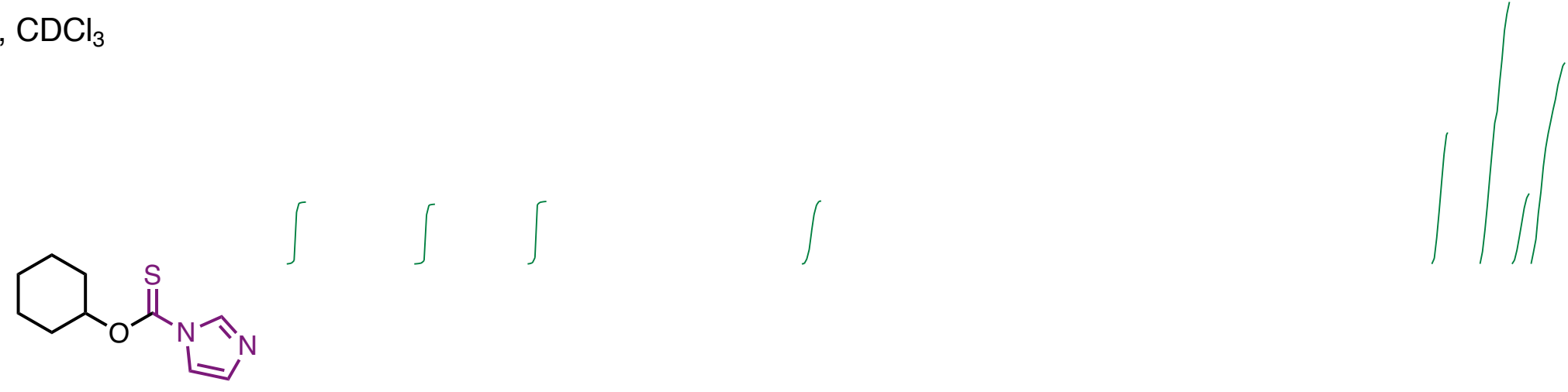

$4 \mathrm{~m}$

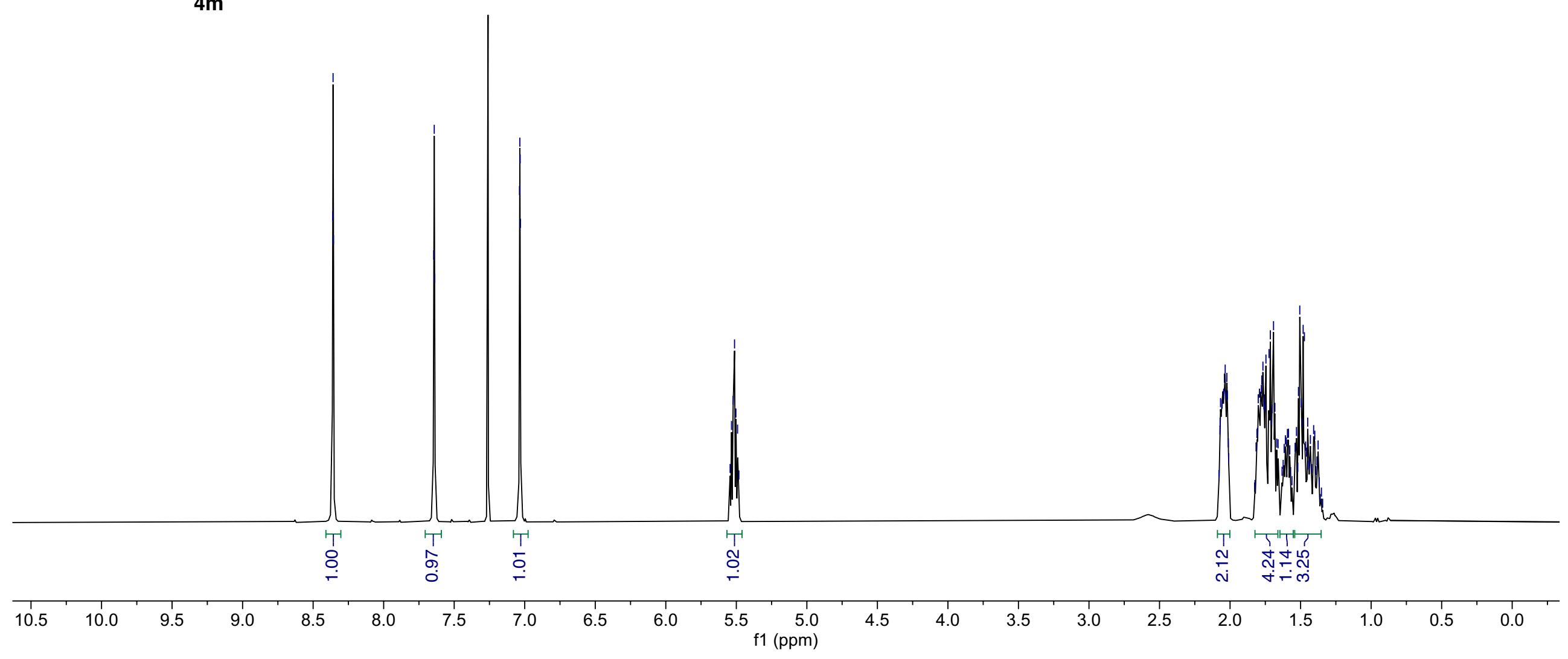




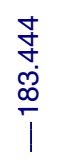

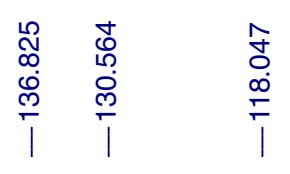

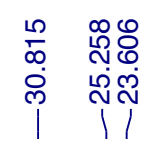

$101 \mathrm{MHz}, \mathrm{CDCl}_{3}$
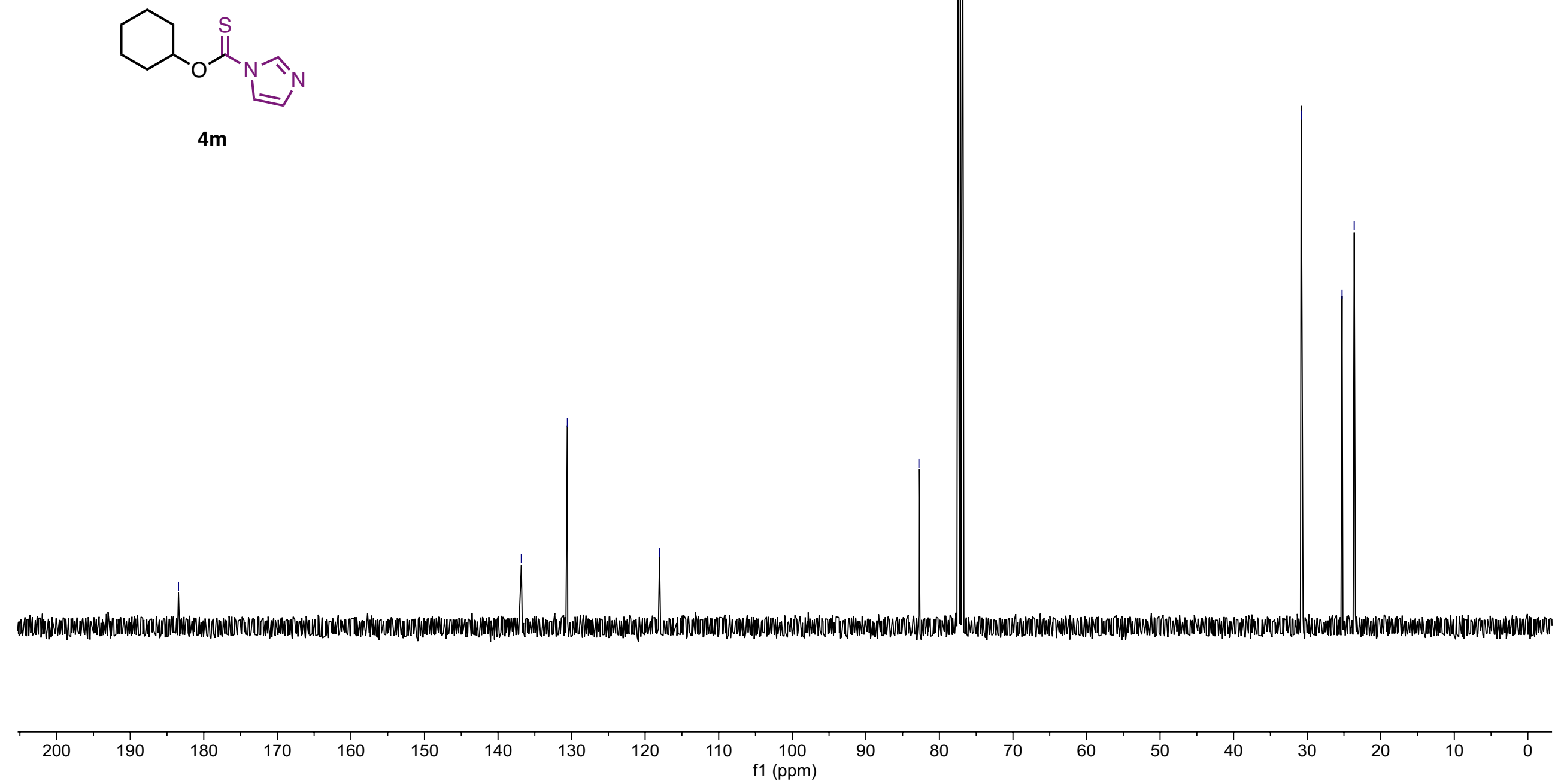

S172 
$400 \mathrm{MHz}, \mathrm{CDCl}_{3}$<smiles>[M]C(OC(=S)n1ccnc1)C(=O)OC</smiles>

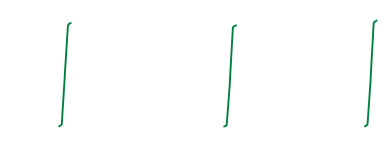

$4 n$
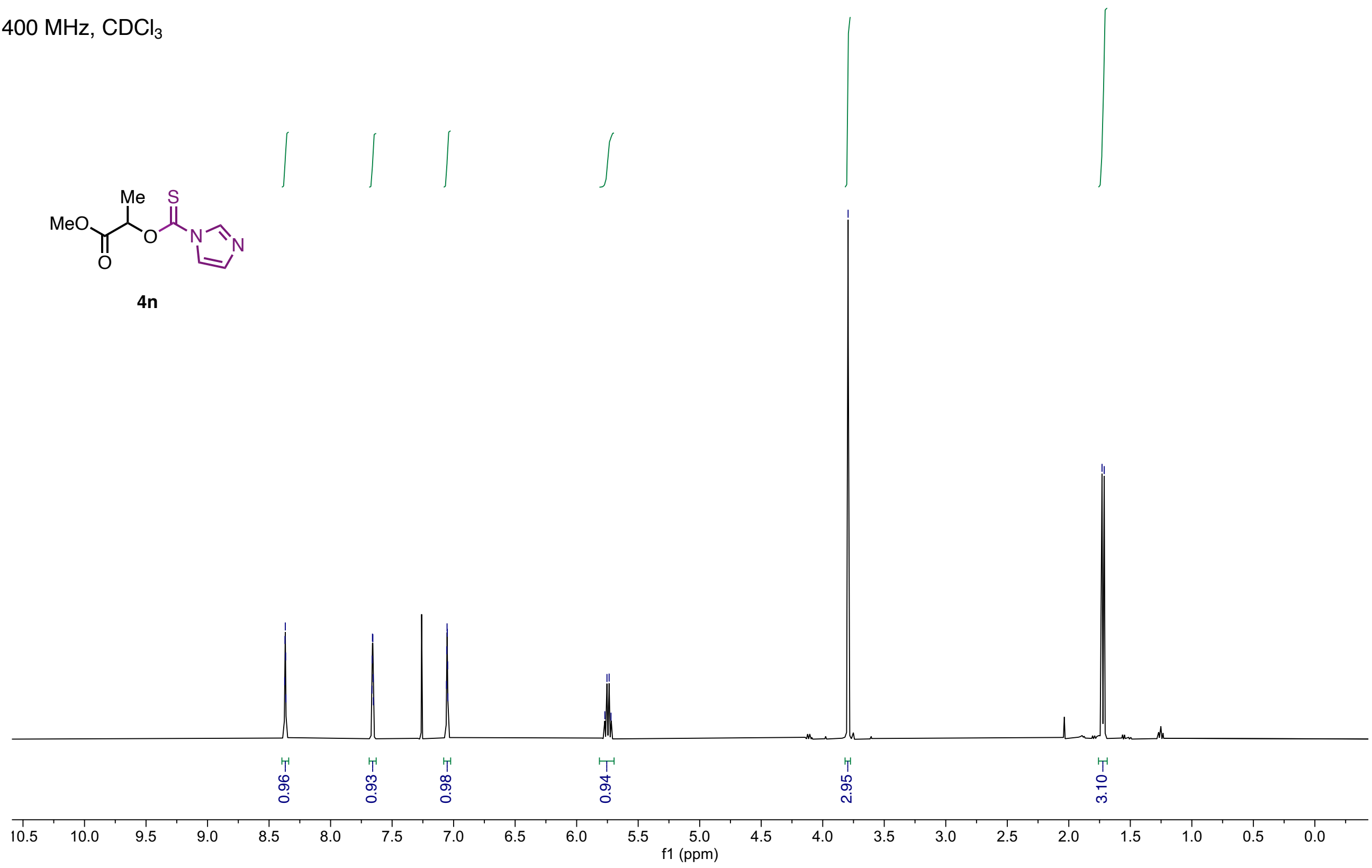
$101 \mathrm{MHz}, \mathrm{CDCl}_{3}$

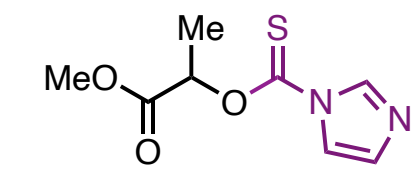

$4 n$

$101 \mathrm{Mz}_{\mathrm{Z}} \mathrm{CDCl}$

(4)

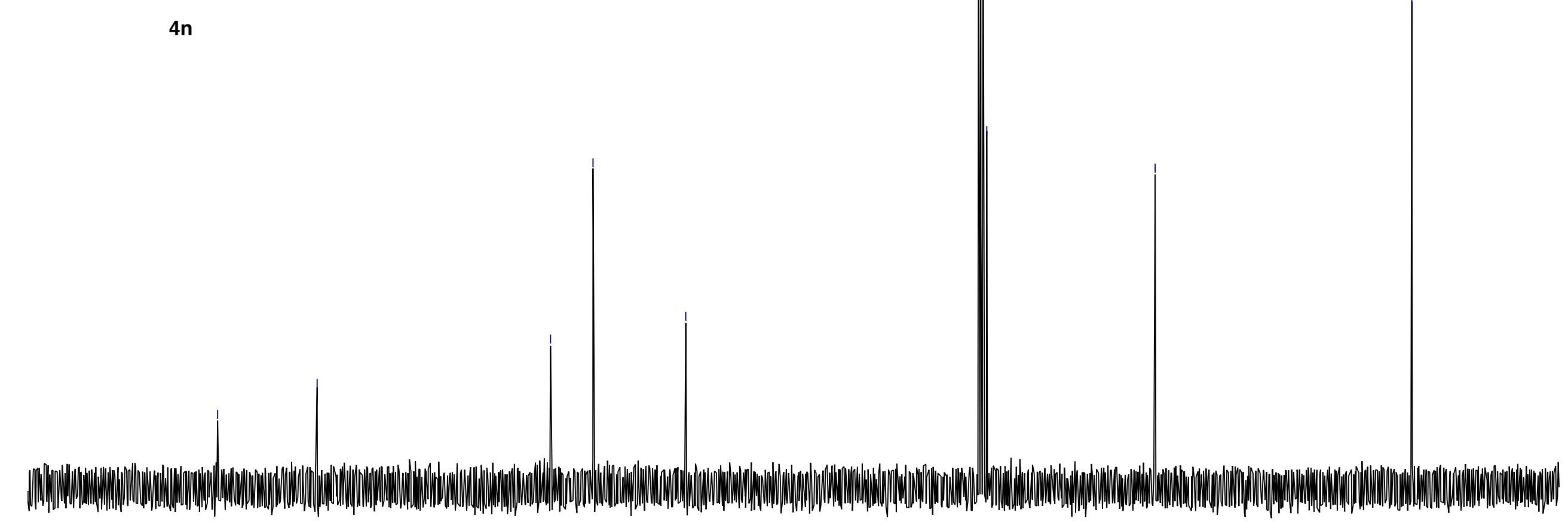

$200 \quad 190,180$

$160 \quad 150 \quad 140$

$130 \quad 120$ $110 \quad \begin{array}{r}100 \\ \mathrm{f} 1(\mathrm{ppm})\end{array}$ $90 \quad 80$ 10
70 $50+40$ $30+20$ $20 \quad 10$ 